
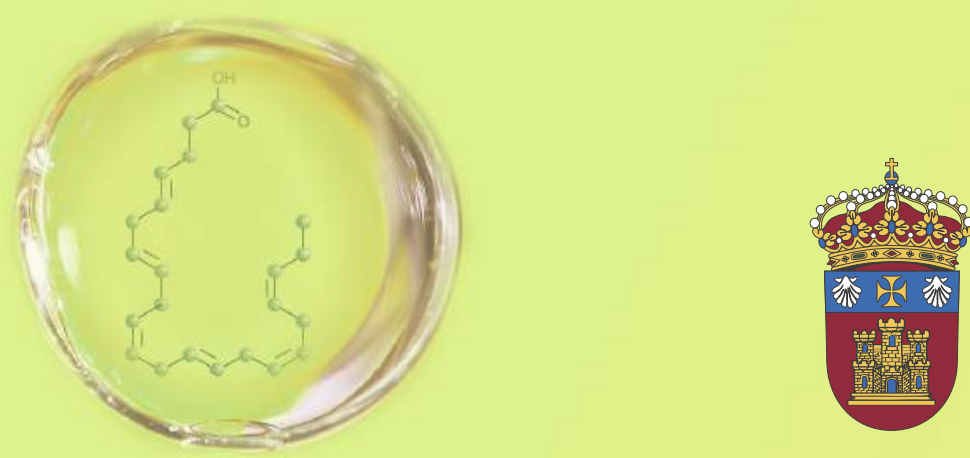

UNIVERSITY OF BURGOS (SPAIN)

DEPARTMENT OF BIOTECHNOLOGY AND FOOD SCIENCE

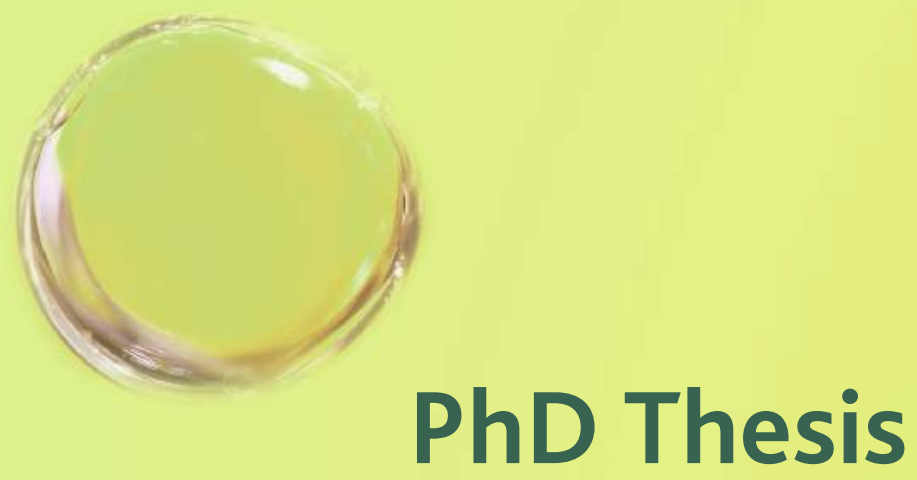

Production of omega-3 fatty acids acylglycerides by lipase-catalyzed glycerolysis of sardine oil in different reaction media

Ángela García Solaesa 



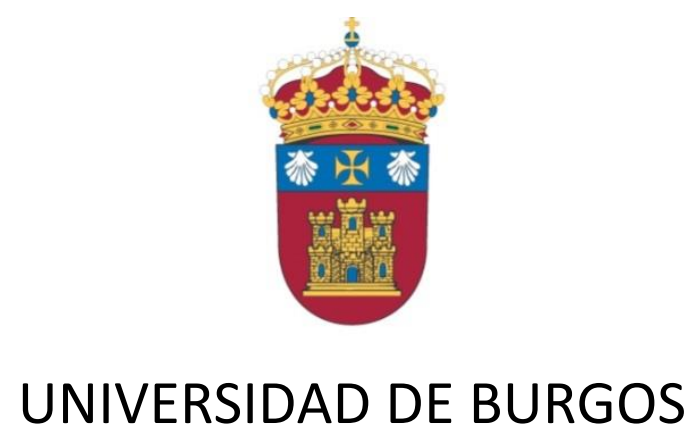

Departamento de Biotecnología y Ciencia de los Alimentos

\section{Production of omega-3 fatty acids acylglycerides by lipase-catalyzed glycerolysis of sardine oil in different reaction media}

\section{TESIS DOCTORAL}

Ángela García Solaesa

Burgos, 2017 



\title{
Production of omega-3 fatty acids acylglycerides by lipase-catalyzed glycerolysis of sardine oil in different reaction media
}

\author{
Memoria que para optar al grado de \\ Doctor por la Universidad de Burgos \\ en el programa Avances en Ciencia y \\ Biotecnología Alimentarias presenta \\ ÁNGELA GARCÍA SOLAESA
}





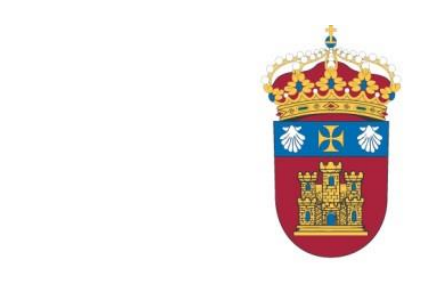

\section{UNIVERSIDAD DE BURGOS}

\section{Departamento de Biotecnología y Ciencia de los Alimentos}

Dña. MARÍA TERESA SANZ DIEZ y Dña. SAGRARIO BELTRÁN CALVo, Profesoras del Departamento de Biotecnología y Ciencia de los Alimentos de la Universidad de Burgos,

\section{CERTIFICAN:}

Que la licenciada Dña. Ángela García Solaesa ha realizado bajo su dirección el trabajo titulado "Production of omega-3 fatty acids acylglycerides by lipase-catalyzed glycerolysis of sardine oil in different reaction media", cuyo título en castellano es: “Producción de acilglicéridos de ácidos grasos omega-3 mediante glicerolisis de aceite de sardina catalizada por lipasas en diferentes medios de reacción".

Considerando que dicho trabajo reúne los requisitos exigidos para ser presentado como Tesis Doctoral, expresan su conformidad con dicha presentación.

Para que conste, firman el presente certificado.

En Burgos, a 28 de Agosto de 2017

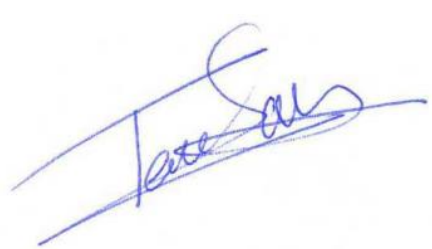

Fdo. María Teresa Sanz Diez

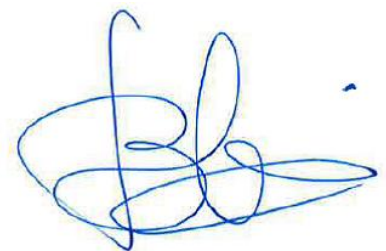

Fdo. Sagrario Beltrán Calvo 



\section{UNIVERSIDAD DE BURGOS}

\section{Departamento de Biotecnología y Ciencia de los Alimentos}

D. JOSÉ MANUEL BENITO MORENO, coordinador del programa de doctorado "Avances en Ciencia y Biotecnología Alimentarias de la Universidad de Burgos,

\section{CERTIFICA:}

Que la memoria titulada "Production of omega-3 fatty acids acylglycerides by lipasecatalyzed glycerolysis of sardine oil in different reaction media" presentada por Dña. Ángela García Solaesa, Licenciada en Ciencia y Tecnología de los Alimentos y Máster en Seguridad y Biotecnologia Alimentarias, ha sido realizada en el Departamento de Biotecnología y Ciencia de los Alimentos bajo la dirección de las Dras. María Teresa Sanz Diez y Sagrario Beltrán Calvo, y en representación de la Comisión Académica del Programa de Doctorado, autoriza su presentación para ser defendida como Tesis Doctoral.

Para que conste, y surta los efectos oportunos, firmo el presente certificado.

En Burgos, a 28 de Agosto de 2017

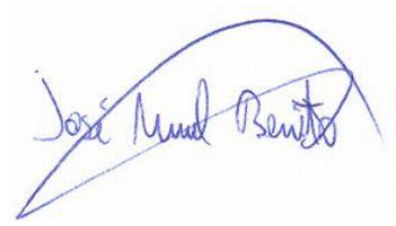

Fdo. José Manuel Benito Moreno

Coordinador del Programa de Doctorado 

Este trabajo ha sido realizado en el marco de los siguientes proyectos de Investigación desarrollados en el seno del Grupo de investigación Biotecnología Industrial y Medioambiental, reconocido por la UBU (GIR-UBU BIOIND) y por la Junta de Castilla y León como Unidad de Investigación Consolidada UIC-128.

- PROCESOS CON FLUIDOS SUPERCRITICOS APLICADOS A LA PRODUCCION Y SEPARACION DE ACILGLICERIDOS ENRIQUECIDOS EN OMEGA-3, financiado por el Ministerio de Economía y Competitividad y el Fondo Europeo de Desarrollo Regional (CTQ2012-39131-C02-01)

- aplicación de tecnologías emergentes a la formulación de COMPUESTOS BIOACTIVOS DE INTERÉS PARA LA INDUSTRIA ALIMENTARIA, financiado por la Junta de Castilla y León y el Fondo Europeo de Desarrollo Regional (BU055U16) 



\section{AGRADECIMIENTOS}

A la Universidad de Burgos por financiar mi contrato predoctoral y al MINECO, la JCyL y Fondos FEDER por financiar los proyectos CTQ2012-39131-C02-01 y BU055U16 en los que he trabajado.

A mis directoras de Tesis. A Tere, por su dedicación, su comprensión y su infinita ayuda. Por ser mucho más que una directora de tesis, por haber estado siempre ahí como una compañera y amiga. A Sagrario, por la confianza que un día puso en mí para comenzar este proyecto. Por sus consejos y su generosidad, y por haber contado conmigo en tantas ocasiones.

A todos los compañeros del Área de Ingeniería Química que han colaborado en esta Tesis. En especial agradecer a Beti todo lo que me ha enseñado, la ayuda que siempre me ha brindado y su confianza. También agradecer a Rodrigo toda su ayuda y disposición con los montajes "supercríticos"; y a Sarai, por haberme echado una mano en esta última etapa, por su disposición y su amistad.

Al Prof. Zheng Guo de la Universidad de Aarhus y a la Dra. Sara Cunha de la Universidad de Oporto, por aceptarme en sus grupos de investigación para realizar mis estancias predoctorales y enseñarme tanto en tan poco tiempo.

A mis amigas Andrea, Raquel, Gloria y Helen; por haber estado ahí, por su interés y por el apoyo que me han brindado.

Y por último, mi más profundo agradecimiento a mis padres, que me lo han dado todo, a mi hermana Virginia y a David, por apoyarme siempre y darme tan buenos consejos. A Dani, por animarme cada día, por su comprensión y por la admiración que me transmite. 

GENERAL INDEX 



\section{GENERAL INDEX}

SUMMARY OF THE THESIS

RESULTS

\section{Chapter 1:}

Liquid-liquid equilibria for systems glycerol + sardine oil + tert-alcohols

\section{Chapter 2:}

Glycerolysis of sardine oil catalyzed by a water dependent lipase in different tert-alcohols as reaction medium .59

\section{Chapter 3:}

Kinetic study and kinetic parameters of lipase-catalyzed glycerolysis of sardine oil in a homogeneous medium

\section{Chapter 4:}

Production and concentration of monoacylglycerols rich in omega-3 polyunsaturated fatty acids by enzymatic glycerolysis and molecular distillation 115

\section{Chapter 5:}

Substrates emulsification process to improve lipase-catalyzed sardine oil glycerolysis in different systems. Determination of lipid oxidation of the reaction products

\section{Chapter 6:}

Effect of temperature on oxidation kinetics and adsorption capacity of commercial immobilized lipases supports to reduce oxidation products in sardine oil.... 


\section{Appendix A:}

Characterization of triacylglycerol composition of sardine oil by using chromatographic techniques

Appendix B:

Analytical methods

REFERENCES 
SUMMARY / RESUMEN 



\section{SUMMARY OF THE THESIS}

Production of omega-3 polyunsaturated fatty acids ( $n-3$ PUFA) concentrates is of great interest for both the pharmaceutical and the food industries. It is well known that the main n-3 PUFA, eicosapentaenoic (EPA) and docosahexaenoic (DHA) acids, have beneficial effects on the prevention and reduction of cardiovascular and inflammatory diseases. However, not all the chemical forms of n-3 PUFA are equal. The bioavailability and susceptibility to oxidation of n-3 PUFA concentrates depend on their molecular structure. Monoacylglycerides and diacylglycerides (MAG and DAG) containing n-3 PUFA present good bioavailability and oxidation stability. Moreover, they present excellent emulsifying properties, being widely used in the food, cosmetic and pharmaceutical industries. Taking into account these considerations and the advantages of enzymatic technology in the formation of this kind of products, this Thesis is focused on the production of MAG rich in n-3 PUFA by a lipase-catalyzed glycerolysis reaction in different reaction systems. The main drawback of this process is the immiscibility of the substrates, therefore the main challenge of this thesis is the evaluation of different possibilities to improve the contact between sardine oil and glycerol to achieve high yields of the reaction products. The use of tert-alcohols, surfactants, supercritical $\mathrm{CO}_{2}$ and a substrate emulsification process are the different alternatives studied in this thesis to carry out the lipase-catalyzed glycerolysis. The effect of different operating variables such as type of lipase, lipase loading, temperature and substrate mole ratio on the kinetic parameters was investigated in the different reaction systems.

In addition to the study of the lipase-catalyzed glycerolysis reaction, in this PhD Thesis a molecular distillation process to fractionate the reaction products and concentrate the MAG rich in n-3 PUFA has been optimized. Different evaporation temperatures were evaluated to maximize the MAG recovery at high purity in the distillate product.

In the final part of this work, the lipid oxidation of sardine oil and reaction products has been evaluated. In this sense, immobilized commercial lipases have showed good adsorption capacity of the oxidation products formed during the glycerolysis reaction. 


\section{RESUMEN DE LA TESIS}

La producción de concentrados de ácidos grasos poliinsaturados omega 3 (AGPI n-3) continúa siendo un tema de interés tanto en la industria farmacéutica como en la alimentaria. Son muy conocidos los efectos beneficiosos en la prevención y reducción de enfermedades de origen cardiovascular e inflamatorio que presentan los principales AGPI $\mathrm{n}$-3, en concreto el ácido eicosapentaenoico (EPA) y el docosahexaenoico (DHA). Ahora bien, no todas las formas químicas de los AGPI n-3 presentan las mismas propiedades. La biodisponibilidad y la susceptibilidad a la oxidación lípidica de los concentrados de AGPI n3 dependen de su estructura molecular, siendo los monoglicéridos y diglicéridos (MAG y DAG) de AGPI n-3 altamente biodisponibles y estables a la oxidación lipídica. Además, presentan excelentes propiedades emulsificantes, por lo que son muy usados en las industrias alimentaria, cosmética y farmacéutica. Teniendo esto en cuenta y las ventajas que presenta la tecnología enzimática en la formación de estos compuestos, esta Tesis Doctoral se ha centrado en el estudio de la producción de MAG ricos en AGPI n-3 mediante glicerolisis catalizada por lipasas abordando diferentes sistemas para llevarla a cabo. El principal inconveniente de este proceso es la inmiscibilidad de los sustratos, por lo que el enfoque de esta tesis es el estudio de diferentes estrategias para mejorar el contacto entre el aceite de sardina y el glicerol para así conseguir altos rendimientos de reacción. Se ha estudiado el uso de tert-alcoholes, surfactantes, $\mathrm{CO}_{2}$ supercrítico y la emulsificación de los sustratos para llevar a cabo la glicerolisis enzimática. En estos sistemas de reacción se ha investigado el efecto de diferentes variables como son el tipo y la cantidad de lipasa, la temperatura de reacción y la relación molar de sustratos.

Además del estudio de la glicerolisis enzimática, otro objetivo de esta Tesis, fue la optimización del proceso de destilación molecular para fraccionar los productos de reacción y concentrar los MAG ricos en AGPI n-3. Se han evaluado diferentes temperaturas de evaporación para maximizar la recuperación de los MAG con alta pureza. También se ha evaluado en esta tesis la oxidación lípidica del aceite de sardina y de los productos de reacción. En este sentido se ha demostrado que las lipasas inmovilizadas son capaces de adsorber los productos de oxidación, tanto primarios como secundarios, formados durante la reacción de glicerolisis. 
INTRODUCTION 



\section{Omega-3 Polyunsaturated Fatty Acids}

Nowadays people are concerned about the importance of having healthy food habits due to the growing knowledge about the important roles that many molecules, naturally found in foods, play in human health and disease prevention. Polyphenols, carotenoids and organosulfur compounds have been extensively studied and their beneficial effects have been scientifically probed (Barba \& Orlien 2017; Davatgaran-Taghipour et al. 2017). Omega-3 polyunsaturated fatty acids ( $n-3$ PUFA) constitute other group of healthy compounds with especial interest. The research in this area has increased continuously during the last 30 years, indeed, omega-3 PUFA are the most investigated nutrients ever (Ciriminna et al. 2017).

Omega-3 refers to the actual location of the first double bond in these unsaturated fatty acids, counting from the carbon on the methyl (omega) end of the compound. The human body cannot form double carbon bonds before the $9^{\text {th }}$ carbon from the omega position, thus essential fatty acids are always compounds with a double bond before this 9th position (Moyad 2005). From a nutritional point of view the three most important $n-3$ PUFA are $\alpha$-linolenic acid (ALA, 18:3 n-3), eicosapentaenoic acid (EPA, 20:5 n-3), and docosahexaenoic acid (DHA, 22:6 n-3) (Figure I.1).
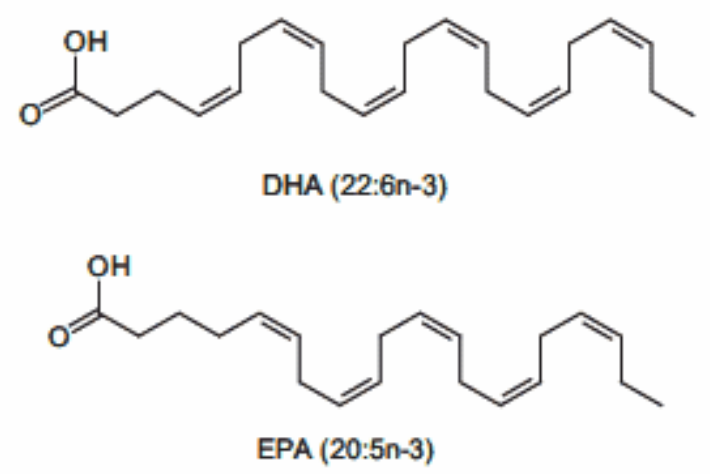

Figure I.1. Molecular structures of cis-4,7,10,13,16,19- docosahexaenoic acid (DHA) and cis-5,8,11,14,17-eicosapentaenoic acid (EPA). 
ALA is essential in human nutrition as precursor for the $n-3$ long chain PUFA. EPA, docosapentaenoic acid (DPA, 22:5 n-3), and to a lesser extend DHA are synthesized from ALA through the sequential action of various desaturases and elongases in animal tissues, but not in plants. The conversion of ALA to EPA and DHA is of interest because the cardioprotective and other beneficial effects of $n-3$ PUFA have been most rigorously studied and closely associated with EPA and DHA. Approximately $8 \%$ to $12 \%$ of dietary ALA is converted to EPA, while the conversion into DHA may be less than 1\% (DeFilippis \& Sperling 2006; Goyens \& Mensink 2006). Therefore dietary intake of n-3 PUFA results essential.

EPA and DHA have demonstrated many cardioprotective effects including antiarrhythmic, blood triglyceride-lowering, antithrombotic effects and reduction of hypertension (Harris 2007; Kris-Etherton et al. 2002). Some research has also shown anti-inflammatory effects including reduction of symptoms of rheumatoid arthritis and Crohn's disease (Calder 2006). They even reduce the risk of developing Alzheimer, depressions, eczema and certain cancer forms (Wani et al. 2015; Wu et al. 2016). Furthermore, the role of DHA in prenatal and postnatal development is well known (Mataix \& Gil 2005). A high intake of DHA has been associated with fewer allergic diseases such as asthma, rhinitis, and eczema in children and mothers, and with a better neural and retinal development of children (Li et al. 2016).

The latest research shows that the most promising health effects of essential fatty acids are achieved through a proper balance between n-3 PUFA and another essential type of fatty acids known as omega-6 polyunsaturated fatty acids (n-6 PUFA) (Ciriminna et al. 2017). A balance between $n-6$ and n-3 PUFA intakes is important because these fatty acids compete for the same enzyme systems involved in elongation and desaturation to synthesize the longer-chain, more unsaturated, and more biologically active fatty acids (Psota et al. 2006). Excessive amounts of $n-6$ PUFA and hence a very high $n-6 / n-3$ ratio of about around 15-20/1, is found in today's Western diets mostly derived from the high consumption of vegetable oils, as such or added in almost every commercially prepared food. A lower ratio of roughly four parts n-6 PUFA to one part n-3 PUFA is more desirable in reducing the risk of many of the chronic diseases of high prevalence in Western 
societies, as well as in the developing countries, although the ideal ratio should be $1 / 1$ (Simopoulos 2002).

Due to the low conversion of ALA into EPA and DHA and the fact that ALA, EPA and DHA may have different biological function, many authorities have separate recommendations for ALA on the one hand, and for EPA and DHA on the other hand. The World Health Organization (WHO) and the Food and Agriculture Organization of the United Nations (FAO) set the population nutrient intake goals for PUFA at 6 to $10 \%$ of total energy, being n-3 PUFA 1 to $2 \%$ of total energy. The recommendation in adults of EPA plus DHA is $250 \mathrm{mg} /$ day, while of ALA is around $2 \mathrm{~g} /$ day (European Food Safety Authority 2010; FAO/WHO 2008).

\section{Fish oil}

The most important natural sources of n-3 PUFA are marine organisms; this is fish, seafood and algae. EPA and DHA are synthetized by marine phytoplankton that feeds, directly or indirectly, these marine organisms. Herring, mackerel, salmon, sardines, tuna, and krill have a fairly good quantity of these compounds (Table I.1). In general, the greasier the fish the greater their concentration of EPA and DHA.

However, some species of fish may contain significant levels of toxic compounds such as methylmercury, polychlorinated biphenyls (PCBs), dioxins, and other environmental contaminants. These substances are present at low levels in fresh waters and oceans, but they bioconcentrate in the aquatic food chain reaching higher values in old, large, predatory fish and marine mammals. Therefore, PCBs and methylmercury have long halflives in the body and can accumulate in people who consume contaminated fish on a frequent basis (Kris-Etherton et al. 2002). Accordingly, the numerous health benefits provided by fish consumption may be compromised by the presence of metals and other toxic compounds if high intakes of large fishes such as tuna and swordfish are consumed (Bosch et al. 2016). Therefore from the contaminant point of view, small oily fish with short life-cycles, such as sardines and anchovies are always preferable. 
Table I.1. Total lipid, n-3 PUFA, EPA and DHA contents (g/100 g) and total amount of $\mathrm{Hg}$ $(\mu \mathrm{g} / \mathrm{g})$ in some species of fish and. Adapted from the literature (Domingo et al. 2007;

Mataix \& Gil 2005).

\begin{tabular}{lccccc} 
Species & Total lipids & n-3 PUFA & EPA & DHA & Total Hg \\
Mackerel & 16.1 & 2.78 & 0.71 & 1.10 & 0.09 \\
Sardine & 8.1 & 2.97 & 1.17 & 1.20 & 0.08 \\
Salmon & 7.8 & 1.85 & 0.55 & 0.86 & 0.05 \\
Tuna & 9.0 & 1.22 & 0.06 & 0.27 & 0.48 \\
Mussel & 2.7 & 0.68 & 0.41 & 0.16 & 0.02 \\
Shrimp & 0.6 & 0.11 & 0.06 & 0.04 & 0.12 \\
\hline
\end{tabular}

Moreover, it is also interesting that some algal and fungal sources of DHA have been recently discovered, without some of the potential contaminants such as methyl mercury found in some fishes (Moyad 2005). Thus seaweed constitutes a vegetarian source of $n-3$ PUFA, but the low lipid content in these marine vegetables $(2 \mathrm{~g} / 100 \mathrm{~g}$ semi-dry sample weight) should be also pointed out (Dawczynski et al. 2007).

Today fish oil is the most popular supplement both in Europe (taken by approximately $20 \%$ of adults) and in the US (Ciriminna et al. 2017). Fish oil is almost exclusively produced from small pelagic species, such as menhaden, sardine, sprat, herring and mackerel; and from liver of white lean fish, mainly cod liver (Van Dijk et al. 2012). Fish oil composition varies markedly depending on the specie and other factors including sex and season (Table I.2). The composition and characteristics of crude fish oil also strongly depend on how the oil is processed and the quality of the raw material.

In general, traditional processes to obtain fish oil involve two stages: oil extraction from raw material and refining. There are many methods to carry out the fish oil extraction. The more traditional ones are heating and pressing small whole fishes, acid and alkaline protein solubilization and conventional liquid extraction with organic solvents. 
Table 1.2. Medium values of main fatty acids typical of fish oils (\%). Adapted from the literature (Young 1986).

\begin{tabular}{lccccc} 
Fatty acid & Menhaden & Sardine & Sprat & Herring & Mackerel \\
Myristic (C14:0) & 9 & 8 & - & 7 & 8 \\
Palmitic (C16:0) & 20 & 18 & 16 & 16 & 14 \\
Palmitoleic (C16:1n-7) & 12 & 10 & 7 & 6 & 7 \\
Oleic (C18:1n-9) & 11 & 13 & 16 & 13 & 13 \\
Gadoleic (C20:1n-11) & 1 & 4 & 10 & 13 & 12 \\
Cetoleic (C22:1n-11) & 0.2 & 3 & 14 & 20 & 15 \\
EPA (C20:5n-3) & 14 & 18 & 6 & 5 & 7 \\
DHA (C22:6n-3) & 8 & 9 & 9 & 6 & 8 \\
\hline
\end{tabular}

This unrefined fish oil is not suitable for human consumption. It contains, to a greater or lesser extent, a wide range of undesirable compounds, such as free fatty acids (FFAs), phospholipids, pigments, lipid oxidation products and environmental pollutants. Therefore it is necessary to include a refining process before obtaining an edible fish oil (Table I.3). The main procedure to remove fish oil impurities involves several steps as degumming, to separate phospholipids; neutralization, to clear free fattyacids and decrease oil acidity; bleaching to absorb pigments orcontaminants and deodorization to remove smelly compounds (Monte et al. 2015). The refined oil obtained as edible fish oil is a complex mixture of triglycerides (TAGs), with around $20-30 \%$ of n-3 PUFA (Solaesa et al. 2014).

Other alternative processes which take place at moderate temperature include enzymatic methods, using food grade proteases to break tissues and cell membranes leading to the release of oil, and supercritical extraction, which is a clean technology used for fish oil extraction from freeze-dried fish muscle and fishery by-products due to its advantages as a green solvent (Rubio-Rodríguez et al. 2012). 
Table I.3. Some characteristics of crude fish oil and refined fish oil.Adapted from Van Dijk et al. (Van Dijk et al. 2012)

\begin{tabular}{lcc} 
Parameter & Crude fish oil & Refined fish oil \\
\hline Peroxide value (meq $\left.\mathbf{~}_{2} / \mathbf{k g}\right)$ & $3-20$ & $<0.1$ \\
Free fatty acids (\% oleic acid) & $1-7$ & $<0.1$ \\
Colour & Up to 14 (Gardner scale) & $<3.0$ Red, 30 Yellow \\
Iron $(\mathrm{mg} / \mathrm{kg})$ & $0.5-7.0$ & $<0.12$ \\
Copper $(\mathrm{mg} / \mathrm{kg})$ & $<0.3$ & $<0.05$ \\
\hline
\end{tabular}

Most of the world fish oil production is used for fish farming, while only approximately $5 \%$ is used to extract its n-3 PUFA contents for use as food ingredient and food supplements (De Meester et al. 2013). Hence, increasing the production of n-3 PUFA rich fish oils has become a good opportunity for valorizing fish by-products and increasing the competitiveness of the fish industry. However, the production of high quality fish oil as source of n-3 PUFA involves, not only searching for n-3 PUFA rich raw materials, but also developing a suitable concentration procedure that could be efficiently carried out at industrial scale (Fiori et al. 2017).

\section{3. n-3 PUFA concentrates}

A popular way to increase n-3 PUFA intake is by available fish oils capsules and foods fortified with n-3 PUFA. These sources can be found both as purified fish oil and as concentrates. The preparation of n-3 PUFA concentrates usually comprises different steps including transesterification, concentration and antioxidant addition. Currently, n-3 PUFA supplements are available in either free fatty acid (FFA), ethyl ester (EE), TAG, diacylglyceride (DAG), monoacylglyceride (MAG) or phospholipid (PL) formulations (Figure I. 2) (Van Dijk et al. 2012; Khaddaj-Mallat et al. 2016). 
TAG
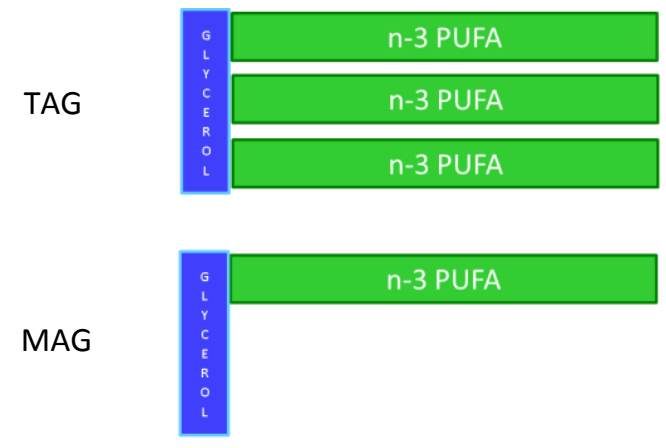

$\mathrm{PL}$

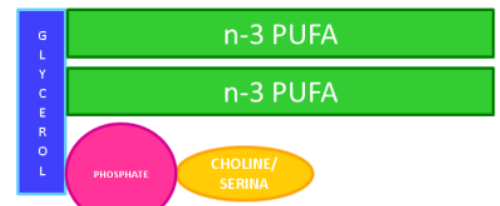

EE

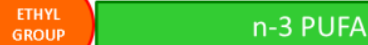

Figure I.2 Molecular structures of different n-3 PUFA formulations (TAG, MAG, PL, EE)

Bioavailability. The influence of the chemical structures on their bioavailability have been widely characterized (Ding et al. 2013; Khaddaj-Mallat et al. 2016; Lawson \& Hughes 1988a; Tou et al. 2011). Lawson et al. (Lawson \& Hughes 1988b) demonstrated in humans that when the fish oil preparations were consumed with a low-fat meal, EPA and DHA as FFAs were completely absorbed, but only two-thirds of the EPA and DHA from the fish oil TAGs and only one-fifth of the EPA and DHA from the fish oil EEs were found to be absorbed. However, different results were found when fish oil preparations were coingested with high-fat meal. In that case, absorption of EPA and DHA from fish oil TAGs increased significantly up to $90 \%$ and absorption of the ethyl esters of both EPA and DHA was increased from 20 to $60 \%$ (Lawson \& Hughes 1988a). Similar results have been observed by Ding et al. (Ding et al. 2013) in their study of the short-term effects of TAG, FFA, EE and PL forms of DHA on its absorption into tissues when mice were fed by highand low- fat diets. They suggested that DHA-PL is safer than the EE and FFA forms of DHA. Moreover, DHA-PL demonstrated good bioactivity, nearly the same as DHA-TAG. So they concluded that DHA-PL and DHA-TAG were more efficient than other DHA forms in 
increasing its concentration in mouse organs. Otherwise, the stereochemistry of fatty acids in acylglycerols does not seem to influence the bioavailability of EPA and DHA (Dyerberg et al. 2010). Dyerberg et al. (Dyerberg et al. 2010) demonstrated a better bioavailability of TAG, especially structured TAG, compared to EEs and FFAs. The bioavailability of EPA+DHA from structured TAG was superior (124\%) even compared with natural fish oil. The superiority of structured TAG over natural TAG could be explained by the hydrolytic activity of pancreatic lipase (Figure I.3) (Dyerberg et al. 2010; Feltes et al. 2013; Khaddaj-Mallat et al. 2016). Once ingested, dietary TAG are hydrolyzed in the small intestine to sn-2 MAGs and FFAs released from the sn-1,3 positions of the original TAG due to the action of the human pancreatic lipase, which is sn-1,3 specific. Therefore, sn-2 MAGs are the most favorable structure for n-3 PUFA to be absorbed by intestinal mucosa (Van Dijk et al. 2012).

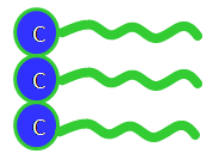

Triglyceride

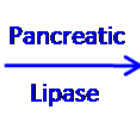

pase

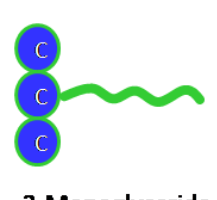

2-Monoglyceride

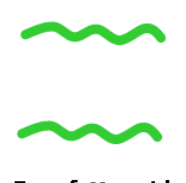

Free fatty acids

Figure I.3 Scheme of hydrolytic activity of pancreatic lipase

The hydrolytic activity of pancreatic lipase is higher towards medium-chain FAs (from 8 to 14 carbon atoms) than for long-chain FAs, especially PUFA. Unfortunately, no natural sources containing Medium-Long-Medium chain FAs in TAG structure have been found. However, these structured lipids can be achieved by enzymatic reactions (Van Dijk et al. 2012). n-3 PUFA from structured TAG can be directly absorbed as sn-2 MAGs after lipase pancreatic digestion, resulting in increasing absorption rates and becoming in a target product for food and pharmaceutical industries. Otherwise, n-3 PUFA concentrates as EE derivatives are poor substrates for pancreatic lipase, hence their absorption is low (Lawson \& Hughes 1988b). On the contrary, although n-3 PUFA in FFA form are absorbed more efficiently than in EE or even in TAG form, FFA could present irritant effects (Ding et al. 2013; Lawson \& Hughes 1988b). 
Oxidative stability. The extremely high susceptibility of n-3 PUFA to oxidative rancidity must be also taken into account. Despite the high nutritional value of these $n-3$ PUFA-rich oils, it is well known that the high susceptibility to oxidation of PUFA, due to their high degree of unsaturation, is the main cause of deterioration and loss of quality of these products. Unfortunately it is difficult to protect them from lipid peroxidation; sometimes this problem is not easily solved by the use of natural antioxidants (Kamal-Eldin \& Yanishlieva 2002). In addition, the oxidizability of lipids rich in PUFA is determined also by the structural organization of these fatty acids in TAG and other lipids. The oxidative stability of n-3 PUFA TAG is influenced by positional distribution. Wijesundera et al. (Wijesundera et al. 2008) established that TAG oxidized more slowly when DHA was located at the sn-2 position (PDP and ODO) compared to the sn-1(3) position (PPD and OOD), as evidenced by slower development of peroxide value, slower depletion of DHA, and slower generation of secondary oxidation products such as propanal and trans, trans-

\section{2,4-heptadienal.}

In case of EE and FFA, some studies have reported that the oxidative stability of n-3 PUFA in EE form is lower than in TAG form, even in presence of antioxidants (Martín et al. 2012); and the n-3 PUFA as FFA are much more prone to auto-oxidation (Ding et al. 2013; Lawson \& Hughes 1988b).

In case of PL, some authors established that n-3 PUFA in PL form has greater stability against lipid oxidation due to their incorporation into cell membranes (AMR 2010). However, Song et al. (Song et al. 2000) reported that rat fed DHA in PL form resulted in similar oxidation as feeding DHA in TAG form. Anyway, krill oil, the main source of $n-3$ PUFA in PL form, contains high amount of a potent antioxidant, astaxanthin, which helps to prevent n-3 PUFA oxidation. The higher drawback is that the production of $n-3$ PUFA in PL form is quite limited since the main source of these PL is krill oil (AMR 2010).

To date, various sources of n-3 PUFA are available with claims that some sources are more beneficial than others. The source of n-3 PUFAs most favorable to health should provide high digestibility and efficient tissue incorporation with the least tissue lipid peroxidation (Tou et al. 2011). Taking these considerations into account, partial acylglycerols (AG), or in a more common term expressed as DAG and MAG, containing n-3 PUFA have potential 
applications as functional ingredients in the food and pharmaceutical industries (Feltes et al. 2013).

\section{Monoacylglycerols}

Extensive research has been applied to the production of new functional ingredients such as MAG and DAG, mainly enriched with n-3 PUFA (Feltes et al. 2013). Some studies have shown that n-3 PUFA as MAG do not require pancreatic lipase to be absorbed, unlike $n-3$ PUFA TAGs which need to be hydrolyzed as FFAs and MAGs prior to intestinal absorption (Cruz-Hernandez et al. 2012; Khaddaj-Mallat et al. 2016). Using pharmacokinetic analysis, a study comparing the effect of PUFA containing MAG, TAG or EE clearly demonstrated that MAG-DHA increases the bioavailability of DHA compared to TAG-DHA or EEDHA (Fortin 2012). Moreover, Morin et al. have widely verified in several studies that MAGEPA, MAG-DHA and MAG-DPA showed beneficial effects in several disorders including chronic airway inflammatory diseases, pulmonary hypertension, rheumatoid arthritis, and lung and colorectal adenocarcinoma (Khaddaj-Mallat et al. 2016; Morin et al. 2014; Morin, Rousseau, et al. 2015; Morin, Fortin, et al. 2013; Morin, Blier, et al. 2015; Morin et al. 2016; Morin, Rousseau, et al. 2013).

Furthermore, MAG and DAG are commercially produced emulsifiers emerging from oleo chemistry. The popularity of partial AG as emulsifiers, especially pure MAG, is due to their dietary safety together with their molecular structure, which combines a hydrophilic and hydrophobic portion. This gives the capability to form a stable and homogenous emulsion in different products where water soluble and water-non soluble compounds are included (Devi et al. 2008). Today, MAG and DAG are widely applied in the food, cosmetic, pharmaceutical and chemical industries. MAGs represent about $70 \%$ of the world wide emulsifier production (Fregolente et al. 2006). Traditionally, MAG and DAG on the global market have been dominated by saturated fatty acid profiles. This is partially due to the uncontrollable damage of the heat-sensitive PUFA structures under the current chemical process performed at very high temperatures $\left(220-260^{\circ} \mathrm{C}\right)$. The chemical catalysis usually applied in the production of MAG and DAG, takes advantage of the low cost of the catalysts and the short reaction times usually applied in this process. However high- 
temperature chemical catalysis leads to oil oxidation, resulting in the development of offflavors and a dark color, which is a particular drawback when dealing with the heatsensitive fish oil. The MAG and DAG obtained by means of chemical catalysis have to be further purified in order to be used in the food industry (Feltes et al. 2013).

In contrast, the recent progress in enzyme technology has made possible more gentle processing methods. Thus, damage of the PUFA rich oils can be avoided due to the much lower temperature required (below $80^{\circ} \mathrm{C}$ ); so that the processing of more heat-sensitive partial AG with PUFA has become feasible (Devi et al. 2008). MAG and DAG containing n-3 PUFA are expected to have plenty application possibilities like incorporation into functional foods and cosmetics, as dietary supplements and as ingredients in pharmaceuticals (Feltes et al. 2013). Different reaction routes have been applied including glycerolysis, hydrolysis, esterificationhave important healthy effects and transesterification reactions like acidolysis a ethanolysis (Devi et al. 2008).

\section{Enzymatic glycerolysis}

The enzymatic glycerolysis seems to be a very promising approach that converts glycerol and fish oils into partial AG containing n-3 PUFA in a simple and relatively cheap way (Figure I.4). For this reaction, the low cost fish oil from fish by-products and the surplus glycerin derived from biodiesel production could be applied as substrates after being purified (Feltes et al. 2013).

The lipase-catalyzed glycerolysis system using an immobilized lipase comprises a hydrophobic oil phase, a hydrophilic glycerol phase, and a solid enzyme phase. In this system, one important drawback is the immiscibility of the reactants, glycerol and oil. However, as the reaction proceeds, the MAG and DAG obtained may act as emulsifiers, improving the contact between both substrates and therefore enhancing the conversion rates. Therefore, the glycerolysis reaction could be carried out by using just the glycerol and the oil as substrates, with no additional solvents or additives, but in this system mass transfer is still limited and the reaction time is long in order to obtain satisfactory conversion (Feltes et al. 2013). 
a)<smiles>[R]C(=O)OCC(COC([R])=O)OC([R])=O</smiles>

Triacylglycerol

Glycerol

Monoacylglycerol

b)<smiles>[R]C(=O)OCC(COC([R])=O)OC([R])=O</smiles>

Triacylglycerol<smiles>OCC(O)CO</smiles>

Glycerol<smiles>[R]C(=O)OCC(O)COC([R])=O</smiles>

Diacylglycerol

Figure I.4. Scheme of glycerolysis reaction to produce MAG (a) and DAG (b). Adapted from Feltes et al. (Feltes et al. 2013).

To overcome the immiscibility of the substrates, one alternative is to use an organic solvent suitable for an efficient glycerolysis reaction. A suitable solvent system to improve the miscibility of substrates will result in an homogeneous system and enhance the conversion of substrate, the reaction rate, and the product distribution in favor of MAG formation (Feltes et al. 2013). Among the different solvents considered in the literature for glycerolysis systems, alcohols with more than four carbons are one of the best options since they contain a polar $\mathrm{OH}$ group and a non-polar carbon chain (Figure I.5).<smiles>CCC(C)(C)O</smiles><smiles>CC(C)(C)O</smiles>

Figure I.5. Molecular structures of tert-pentanol (TP, left) and tert-butanol (TB, right) 
Alcohols are competitors to glycerol; however, tertiary alcohols, due to their tertiary structure, that make them to have a strong steric hindrance for the enzymatic reaction, do not involve in reactions with fatty acids (Yang et al. 2005a). According to Damstrup et al. (Damstrup et al. 2005) the relative low log $P$ values of tert-butanol (0.35) and tertpentanol (0.89) indicate both hydrophilic and hydrophobic characteristics. This fact makes them suitable solvents for both, oil and glycerol, offering high reaction yields in short reaction times. However, one must take into account that organic solvents are usually expensive and may be toxic. Organic solvents may also produce several undesirable physicochemical effects on the enzyme molecules and also require separation steps for solvent removal, increasing the process costs (Feltes et al. 2013).

Another possibility to increase the interfacial area is to use a surfactant. A surfactant is a molecule with amphipathic characteristics capable of forming micellar systems. The formation of a microemulsion of the reactants (glycerol-in-oil) as reverse micelles can help to improve mass transfer rates. Furthermore, lipases demonstrate high interfacial activity in micelle systems and reaction occurs at the interface between the substrates and the enzyme. In addition, a reaction system containing a surfactant, but with no organic solvents, can be considered as a "green" and low cost technology. Also applying ultrasound irradiation has been proposed to reduce mass transfer limitation (Fiametti et al. 2012; Valério et al. 2010).

Another alternative to avoid organic solvents in glycerolysis reactions can be compressed fluids (Moquin et al. 2005) and supercritical fluids (SCFs) used as reaction media. Supercritical carbon dioxide $\left(\mathrm{SC}-\mathrm{CO}_{2}\right)$ is probably the most used SCF due to its numerous benefits (non-toxic, non-flammable, readily available at high purities and low costs, and relatively mild critical conditions). Enzymatic concentration of $n-3$ PUFA in supercritical fluids (SCFs) is an interesting option for the prevention of oxidation during processing of fish oil (Lin \& Chen 2008). Besides, SC- $\mathrm{CO}_{2}$ can be easily separated from the reaction products by simple depressurization and allows fractionation of the reaction products. In case of enzymatic glycerolysis, other compressed fluids such as propane, $\mathrm{n}$-butane, and acetone, have been used (Esmelindro et al. 2008; Tai \& Brunner 2011; Valério et al. 2010). 
Some studies of glycerolysis of vegetable oils in $\mathrm{SC}-\mathrm{CO}_{2}$ at high temperatures can be found but with no enzymatic catalyst (Moquin et al. 2005; Temelli et al. 1996).

The enzymatic process occurs under mild conditions ( $\mathrm{pH}$ and temperature), resulting in reduced energy consumption and thermal degradation of substrates and products, which imply a reduction in the formation of oxidation compounds. This factor is fundamental when working with an oil rich in PUFA, which are extremely heat-sensitive (Feltes et al. 2013). Despite the advantages of enzymatic catalysis, the cost of the lipases is an important point to take into account for large-scale, however using immobilized lipases the cost is reduced since it allows the recovery of the enzyme for subsequent reuses (Brady et al. 1988). The most widely immobilized commercial lipases used in enzymatic glycerolysis are from Candida antarctica (Novozym435 and Lipozyme 435), Thermomyces lanuginosus (Lipozyme TL IM) and Rhizomucor miehei (Lipozyme RM IM) (Table I.4). Lipozyme 435 can act on different substrates such as vegetable oils (Damstrup et al. 2005; Krüger et al. 2010; Yang et al. 2005a) and fish oil (Feltes et al. 2010; Torres et al. 2002). Likewise, it is also capable of acting in the presence of solvents such as tert-alcohols (Damstrup et al. 2005; Yang et al. 2005a) and in the presence of surfactants such as Tweens, Triton X-100 and AOT (Valério, Fiametti, et al. 2009; Valério et al. 2010). Although this commercial lipase is very flexible and robust, it has a high cost, and therefore there is a special interest in the study of alternative biocatalysts, reaction systems, and processes for MAG and DAG production.

\section{Purification of $n-3$ PUFA}

The n-3 PUFA in highly purified form are not easily prepared by any single fractionation technique due to the complex composition of marine oils. It depends on the chemical form of the $n-3$ PUFA, the desired concentration and their purity in the final product (Breivik et al. 1997). Currently combined techniques are used (Fiori et al. 2017).

Several methods have been reported for concentrating n-3 PUFA derivatives, but only a few are suitable for large-scale production. The available technologies include molecular distillation, also known as short path distillation (SPD), low-temperature crystallization, 
urea complexation and supercritical fluid fractionation. The main properties of each acid or acid group, namely boiling and melting point differences, molecular size and degree of unsaturation are considered in developing separation techniques. Each method has its own advantages and drawbacks (Table I.5), but currently, molecular distillation is the most industrially used process for n-3 PUFA separation and concentration (Wanasundara 2011). Separation of fatty acids by distillation depends on the relative volatility of individual fatty acids in a mixture. This method takes advantage of differences in the boiling point and molecular weight of fatty acids under reduced pressure. The boiling point of a mixture of fatty acids varies significantly with the chain length of the fatty acids involved. This fact is used in fractional distillation as a means of separating short- and long-chain fatty acids (Berger \& McPherson 1979). In molecular distillation, compounds with different boiling points are separated under vacuum, which decreases the evaporation temperature and minimizes the residence time enabling heat-sensitive compounds to be separated with minimal thermal degradation. Therefore, this technology has been widely used in lipid areas, for instance, in purification of structured lipids, separation of MAG from acylglycerol mixtures, extraction of valuable FFA from vegetable oil, recovery of polyphenols and tocopherols, etc. (Fregolente et al. 2010; Yeoh et al. 2014; Zha et al. 2014).

Moreover, the melting point of fatty acids changes considerably with the degree of unsaturation and this could be used to separate a mixture of fatty acids into their saturated and unsaturated components. By changing the temperature of the mixture, fatty acids can be separated according to the degree of unsaturation at their respective crystallization temperatures. The low-temperature crystallization process may be carried out on the neat liquid in the absence of a solvent or in a selected solvent mixture, being the last one more effective. At low temperatures, long chain saturated fatty acids which have higher melting points crystallize out and PUFA remain in the liquid form (Shahidi \& Wanasundara 1998). This method requires the least number of steps and the simplest equipment. However, crystallization at low temperature has some technical limitations, such as molecular association and mixed-crystal formation and use of large quantities of organic solvents (Van Dijk et al. 2012). 
Table 1.4. Conditions and results for some of recent studies on enzymatic glycerolysis reactions.

\begin{tabular}{|c|c|c|c|c|}
\hline Lipase & Substrate & Conditions & Products & Reference \\
\hline Novozym & Virgin coconut & $50^{\circ} \mathrm{C}, 5 \%$ of enzyme, & $3.3 \% \mathrm{MAG}$ & (Derawi et al. \\
\hline 435 & oil & $1: 1(\mathrm{G}: O), 24 \mathrm{~h}$ & $3.6 \% \mathrm{DAG}$ & 2017) \\
\hline $\begin{array}{l}\text { CALB on } \\
\text { SBA-15 }\end{array}$ & Corn oil & $\begin{array}{c}50^{\circ} \mathrm{C}, 5 \% \text { of enzyme, } \\
\text { TP, } 1: 2(\mathrm{G}: \mathrm{O}), 12 \mathrm{~h}\end{array}$ & $\begin{array}{l}\text { 15\% MAG, } \\
53.3 \% \text { DAG }\end{array}$ & $\begin{array}{l}\text { (Cai et al. } \\
\text { 2016) }\end{array}$ \\
\hline $\begin{array}{l}\text { Candida } \\
\text { rugosa }\end{array}$ & Olive oil & $\begin{array}{l}40^{\circ} \mathrm{C}, 2 \% \text { of enzyme, } \\
\text { isopropanol:TB (1:3), } \\
\qquad 2: 1(\mathrm{G}: \mathrm{O}), 4 \mathrm{~h}\end{array}$ & $\begin{array}{l}55.8 \% \text { MAG, } \\
\text { 16.4\% DAG }\end{array}$ & $\begin{array}{c}\text { (Singh \& } \\
\text { Mukhopadhy } \\
\text { ay 2016) }\end{array}$ \\
\hline $\begin{array}{c}\text { Novozym } \\
435\end{array}$ & $\begin{array}{l}\text { Soybean and } \\
\text { canola oils }\end{array}$ & $\begin{array}{c}70^{\circ} \mathrm{C}, 10 \% \text { of enzyme, } \\
\text { ultrasound, } 0.8: 1 \\
(\mathrm{G}: \mathrm{O}), 2 \mathrm{~h}\end{array}$ & $\begin{array}{c}\text { 65-75\% } \\
\text { MAG+DAG }\end{array}$ & $\begin{array}{l}\text { (Remonatto } \\
\text { et al. 2015) }\end{array}$ \\
\hline $\begin{array}{l}\text { Lipozyme } \\
\text { TL }\end{array}$ & Fish oil & $\begin{array}{c}40^{\circ} \mathrm{C}, 25 \% \text { of enzyme, } \\
\text { Tween } 65,0.5: 1(\mathrm{G}: 0) \text {, } \\
6 \mathrm{~h}\end{array}$ & $20.8 \%$ DAG & $\begin{array}{l}\text { (Monte } \\
\text { Blanco et al. } \\
\text { 2015) }\end{array}$ \\
\hline
\end{tabular}

Molecular configuration of fatty acids is also utilized in developing separation techniques, especially in urea complexation. Urea has the unique property to form solid complexes (adduct) with straight-chain hydrocarbons. However, the presence of cis double bonds in the carbon chain increases the bulk of the molecule and reduces the likelihood of its complexation with urea. This method presents some technical advantages in fractionation of $n-3$ PUFA such as high efficiency and better selectivity. However, if the starting material for urea fractionation contains residual amounts of phospholipids or partial acylglycerols, the formation of urea complexes will be greatly reduced. In the process of urea complexation, the oil (acylglycerols) is first split into fatty acids using $\mathrm{KOH}$ or $\mathrm{NaOH}$ (Shahidi \& Wanasundara 1998). Alternatively, this procedure can be carried out using 
methyl or ethyl esters of fatty acids rather than free fatty acids, but the yield will be lower. The main disadvantage of this method is that ethyl carbamate, an animal carcinogen, can be formed by the reaction of urea with ethanol, so the use of urea for concentrating PUFAs for human consumption should be avoided (Canas \& Yurawecz 1999).

Supercritical fluid fractionation (SFF) is a relatively new separation process that may avoid some of the problems associated with the use of conventional separation techniques. The conventional methods of $n-3$ PUFA concentration requires high temperatures which result in loss or decomposition of the thermally labile compounds and also leave the toxic residues of solvents in the n-3 PUFA concentrates (Staby \& Mollerup 1993). The transport properties such as viscosity and diffusivity of supercritical fluids are generally gas-like, more favorable than those of typical liquids. Hence, because of their properties, supercritical fluids offer an attractive choice for extraction and fractionation of a variety of raw materials. For food commodities, $\mathrm{CO}_{2}$ is chosen because of its moderate critical temperature and pressure $\left(31.1^{\circ} \mathrm{C}, 73.8 \mathrm{bar}\right)$ and because it is inert, inexpensive, nonflammable, environmentally acceptable, readily available and safe. The separation of PUFA by SFF is dependent on the molecular size of the components involved rather than their degree of unsaturation; therefore, a prior concentration step is needed to achieve a high concentration of PUFA in the final product (Mishra et al. 1993). In case of supercritical fluid fractionation, most studies are directed towards fractionating the FFAs or their esters, which are more soluble in supercritical fluids. To design an efficient process, more knowledge on the solubility and phase equilibria of other compounds in the supercritical fluid is still needed (Van Dijk et al. 2012). 
Table I.5. Comparison of most common omega-3 concentration techniques. Adapted from De Meester et al.(De Meester et al. 2013)

\begin{tabular}{|c|c|c|c|c|}
\hline Characteristic & $\begin{array}{l}\text { Molecular } \\
\text { distillation }\end{array}$ & $\begin{array}{l}\text { Low- } \\
\text { temperature } \\
\text { crystallization }\end{array}$ & $\begin{array}{c}\text { Urea } \\
\text { complexation }\end{array}$ & $\begin{array}{l}\text { Supercritical } \\
\text { Fluid } \\
\text { Fractionation }\end{array}$ \\
\hline $\begin{array}{l}\text { Selective } \\
\text { towards }\end{array}$ & Boiling point & Melting point & Saturated fats & Chain length \\
\hline Operation $\mathbf{T}$ & $140-220^{\circ} \mathrm{C}$ & 0 to $-70^{\circ} \mathrm{C}$ & -10 to $90^{\circ} \mathrm{C}$ & $35-50^{\circ} \mathrm{C}$ \\
\hline Operation p & $0.001 \mathrm{mbar}$ & 1 bar & 1 bar & $>140 \mathrm{bar}$ \\
\hline Toxic solvents & No & Possible & No & No \\
\hline Max EPA/DHA & $65-75 \%$ & $>90 \%$ & $45-65 \%$ & $75-85 \%$ \\
\hline $\begin{array}{l}\text { Decontamina- } \\
\text { tion efficacy }\end{array}$ & Very high & Low & Low & Medium \\
\hline $\begin{array}{l}\text { Mode } \\
\text { operation }\end{array}$ & Continuous & Batch & Batch & Continuous \\
\hline $\begin{array}{l}\text { Risk of } \\
\text { oxidation }\end{array}$ & Low & Possible & Possible & Low \\
\hline $\begin{array}{l}\text { Capital } \\
\text { Investment }\end{array}$ & Low & Low & Low & High \\
\hline
\end{tabular}


OBJECTIVES 



\section{OBJECTIVES}

The main objective of this work is to present new experimental information concerning the effect of relevant process parameters related to the production of monoacylglycerols (MAG) rich in omega 3 polyunsaturated fatty acids (n-3 PUFA) by enzymatic glycerolysis of sardine oil. The substrates of this reaction, glycerol and oil, are immiscible. Different strategies have been developed in this work to solve this drawback.

Along the chapters, different media and lipases are evaluated to carry out a lipasecatalyzed glycerolysis of sardine oil in order to produce in high yield partial acylglycerols, mainly MAG, rich in n-3 PUFA. The main targets of the present work are summarized below:

Firstly, organic solvents, tert-alcohols, were studied as reaction media for the glycerolysis reaction. Chapters $1-4$ evaluate the use of tert-alcohols in lipasecatalyzed glycerolysis of sardine oil.

○ Chapter 1 "Liquid-liquid equilibria for systems glycerol + sardine oil + tert-alcohols": phase equilibrium between the substrates, glycerol and sardine oil, and tert-alcohol, at two different temperatures, 30 and $50^{\circ} \mathrm{C}$, was determined to carry out efficiently the glycerolysis reaction.

○ Chapter 2 "Glycerolysis of sardine oil catalyzed by a water dependent lipase in different tert-alcohols as reaction medium": Lipozyme RM from Rhizomucor miehei was studied to catalyze the glycerolysis reaction of sardine oil in tert-butanol and tert-pentanol. Different reaction conditions were evaluated in these systems to optimize the reaction yield.

○ Chapter 3 "Kinetic study and kinetic parameters of lipase-catalyzed glycerolysis of sardine oil in a homogeneous medium": Tert-butanol as reaction medium and Lipozyme 435 from Candida antarctica as lipase were studied in this chapter to optimize the glycerolysis reaction of sardine oil. 
- Chapter 4 "Production and concentration of monoacylglycerols rich in omega-3 polyunsaturated fatty acids by enzymatic glycerolysis and molecular distillation": In this chapter, glycerolysis reaction was carried out in tert-pentanol as reaction medium using the commercial immobilized lipase Lipozyme 435 as biocatalyst. Molecular distillation was used to fractionate the reaction products obtained at the optimized reaction conditions in order to concentrate the MAG rich n-3 PUFA fraction.

Secondly, to avoid the use of organic solvents, other alternatives were evaluated to carry out the lipase-catalyzed glycerolysis of sardine oil.

- Chapter 5 "Substrates emulsification process to improve lipasecatalyzed sardine oil glycerolysis in different systems. Determination of lipid oxidation of the reaction products": the pre-emulsification of the substrates in solvent free system and the use of surfactants and $\mathrm{SC}-\mathrm{CO}_{2}$, as green solvent, were evaluated in lipase-catalyzed glycerolysis of sardine oil. Moreover, the lipid oxidation of the reaction products was also determined in this chapter.

Finally, regarding lipid oxidation and the high interest of reducing the oxidation products in n-3 PUFA rich oils, Chapter 6 was included in this thesis.

○ Chapter 6 "Effect of temperature on oxidation kinetics and adsorption capacity of commercial immobilized lipases supports to reduce oxidation products in sardine oil": in this chapter it was demonstrated that at temperatures of $90^{\circ} \mathrm{C}$, the supports of the commercial immobilized lipases usually used in enzymatic reactions have the capacity of adsorpt both primary and secondary oxidation products formed during lipase-catalyzed reactions.

Additionally, in order to characterize the raw material used in this work, the TAG composition of the initial sardine oil was also determined. Appendix A includes this analytical method which is a part of a scientific publication. 
RESULTS 



\section{RESULTS}

The more outstanding results of this PhD Thesis are presented as different chapters which correspond to the scientific publications detailed below. A brief summary in Spanish language of each publication is included at the beginning of each chapter.

Chapter 1: Liquid-liquid equilibria for systems glycerol + sardine oil + tert-alcohols.

Chapter 2: Glycerolysis of sardine oil catalyzed by a water dependent lipase in different tert-alcohols as reaction medium.

Chapter 3: Kinetic study and kinetic parameters of lipase-catalyzed glycerolysis of sardine oil in a homogeneous medium.

Chapter 4: Production and concentration of monoacylglycerols rich in omega-3 polyunsaturated fatty acids by enzymatic glycerolysis and molecular distillation.

Chapter 5: Substrates emulsification process to improve lipase-catalyzed sardine oil glycerolysis in different systems. Determination of lipid oxidation of the reaction products.

Chapter 6: Effect of temperature on oxidation kinetics and adsorption capacity of commercial immobilized lipases supports to reduce oxidation products in sardine oil. 



\section{CHAPTER 1}

\section{Liquid-liquid equilibria for systems glycerol + sardine oil + tert-alcohols}

Á. García Solaesa, S.L. Bucio, M.T. Sanz, S. Beltrán and S. Rebolleda (2013).

"Liquid-liquid equilibria for systems glycerol + sardine oil + tert-alcohols".

Fluid Phase Equilibria, 356, 284-290 



\section{Capítulo 1}

\section{Equilibrio líquido-líquido en los sistemas glicerol + aceite de sardina + tert-alcoholes}

\section{Resumen}





\section{Abstract}

Monoacylglycerols (MAGs) can be produced by lipase-catalyzed glycerolysis of oils and fats at atmospheric pressure and low temperature. The use of organic solvents as reaction media helps to create a homogeneous reaction system between the immiscible reactants glycerol and oil. In this work liquid-liquid equilibrium at two different temperatures (303.2 and $323.2 \mathrm{~K}$ ) and at atmospheric pressure has been determined for two solvent-systems in the glycerolysis of fish oil (sardine oil): glycerol + sardine oil + tert-butanol and glycerol + sardine oil + tert-pentanol. From the experimental solubility (binodal) curves and tie-lines, it could be observed that the system mutual solubility does not significantly increaseby increasing temperature from 303.2 to $323.2 \mathrm{~K}$. The Othmer-Tobias correlation was used to analyze the consistency of the tie-line data. The experimental liquid-liquid data were correlated satisfactorily by using the NRTL model for the activity coefficient calculation. $\varkappa^{\circ}$

\section{Introduction}

Fish oil is one of the main sources of omega 3 polyunsaturated fatty acids ( $n-3$ PUFA), specially eicosapentaenoic acid (20:5 n-3, EPA) and docosahexaenoic acid (22:6n-3, DHA). These compounds have been reported to have beneficial effects on cardiovascular diseases, reduction of blood pressure and plasma triglyceride levels, and control of overactive immune functions (Üstün et al. 1997). Glycerolysis of fats and oils is often carried out to concentrate these PUFA in their natural monoacylglycerides form (2-MAG). The enzymatic catalysis in non-aqueous media using lipases is a usual method for synthesizing structured lipids. Lipase-catalyzed glycerolysis of oils using 1,3-specific lipases has been shown as an interesting alternative to the chemical methods due to the mild reaction conditions for reactions involving the highly unstable $n-3$ polyunsaturated fatty acids (Haraldsson et al. 1995). For this bioconversion it is necessary to introduce a solvent in the reaction system to improve the solubility of the reactants, oil and glycerol. Since the work of Zaks and Klibanov (Zaks \& Klibanov 1985), organic solvents have been employed extensively in enzymatic reactions. Among the different solvents considered in the literature for glycerolysis systems, alcohols with more than five carbons are one of the best options since they contain a polar $-\mathrm{OH}$ group and a nonpolar carbon chain. Since 
alcohols are competitors to glycerol, tertiary alcohols are considered because of its tertiary structure that makes them to have a strong steric hindrance for the enzymatic reaction (Yang et al. 2005a). According to Damstrup et al. (Damstrup, Abildskov, et al. 2006) the relative low log $P$ values of tert-butanol and tert-pentanol indicate both hydrophilic and hydrophobic characteristics, with predominant hydrophilic characteristics. This fact makes them suitable solvents for both, oil and glycerol.

Knowledge of the phase behavior for systems containing fish oil, glycerol and the solvent added as reaction media is important for a correct design of the glycerolysis process since this can influence the reaction pathway as well as the further purification steps (Lanza et al. 2009).

Composition of the oil from individual fish species varies, depending on its diet, time of the year and location in the same way as do the oils from vegetable sources (Young 1986) Fish oil is a multicomponent mixture. In the refined process, polar lipids, mainly phospholipids, free fatty acids, and other minor compounds are removed. A neutral lipid analysis for the sardine oil used in this work shows that nearly $99.5 \%$ of the sardine oil are triacylglycerols (TAG). In spite of difference in TAG composition among sardine oil species, determination of phase equilibrium data on natural mixtures is important to estimate the proper process conditions. In literature, phase equilibrium studies concerning vegetable oils are more abundant; however phase equilibrium studies involving mammals or fish oil are scarce.

This work presents liquid-liquid equilibrium data for two ternary systems in the glycerolysis of sardine oil: glycerol + sardine oil + tert-butanol and glycerol + sardine oil + tert-pentanol at $303.2 \mathrm{~K}$ and $323.2 \mathrm{~K}$. Binodal curves were obtained by the cloud-point method. Tie-lines have been directly determined by using a high temperature chromatograph capillary column (HT-GC). The results were compared with indirect measurements of tie lines through density measurement of the two phases. The Othmer Tobias equation was applied to confirm the reliability of experimentally measured tie line data. The experimental data were correlated by the nonrandom two-liquid (NRTL) activity coefficient model, using the simplex minimization method with a weight compositionbased objective function. 


\section{Experimental section}

\subsection{Materials}

Glycerol was purchased from Sigma Aldrich with a purity of $>99.5 \%$ and a water content of $0.04 \%$. Tert-butanol and tert-pentanol were purchased from Merck with a purity of $\geq 99 \%$ and a water content of $0.298 \pm 0.033 \%$ and $0.065 \pm 0.023 \%$ respectively. Refined sardine oil was kindly provided by Industrias Afines S.L. Densities of the compounds were measured by using an Anton Paar DMA 5000 and are presented in Table 1.1 together with some values found in the literature (Domanska 1998; Riddick et al. 1986).

Table 1.1. Density of the pure components. ${ }^{a}$

\begin{tabular}{|c|c|c|c|}
\hline \multirow{2}{*}{ Component } & \multicolumn{2}{|c|}{$\rho_{\text {exp. }} / \mathrm{kg} \cdot \mathrm{m}^{-3}$} & \multirow{2}{*}{$\rho_{\text {literature }} / \mathrm{kg} \cdot \mathrm{m}^{-3}$} \\
\hline & $303.2 \mathrm{~K}$ & $323.2 \mathrm{~K}$ & \\
\hline Glycerol & 1252.11 & 1240.28 & $\begin{array}{l}1255.12^{303.15} \\
\text { (Riddick et al. } \\
1986)\end{array}$ \\
\hline \multirow[t]{2}{*}{ Tert-butanol } & 775.48 & 754.03 & $\begin{array}{l}775.45^{303.15} \\
\text { (Riddick et al. } \\
\text { 1986) }\end{array}$ \\
\hline & & & $\begin{array}{l}775.85^{303.15} \\
\text { (Domanska 1998) }\end{array}$ \\
\hline Tert-pentanol & 801.33 & 783.30 & $\begin{array}{l}805.0^{298.15} \text { (Riddick } \\
\text { et al. } 1986)\end{array}$ \\
\hline Sardine oil & 922.18 & 908.42 & --- \\
\hline
\end{tabular}

${ }^{\mathrm{a}}$ Standard uncertainty $u$ is $u(\rho)=0.05$.

\subsection{Apparatus and procedure}

Binodal curves. The binodal curve of the two ternary systems studied in this work was determined at $303.2 \pm 0.5 \mathrm{~K}$ and $323.2 \pm 0.5 \mathrm{~K}$ and atmospheric pressure by turbidimetric analysis using the titration method. Different binary mixtures of sardine oil + tertiary alcohol and glycerol + tertiary alcohol have been prepared at various concentrations by using an analytical balance (Sartorius Basic, accurate $\pm 0.0001 \mathrm{~g}$ ). These binary mixtures 
were titrated with the third component (glycerol or sardine oil) by using a syringe needle until a change from transparent to turbid was observed by using a turbidimeter (Eutech Instruments TN-100). The uncertainty of the drop has been estimated to be $\pm 0.005 \mathrm{~g}$ for glycerol and $\pm 0.0018 \mathrm{~g}$ for sardine oil. The liquid mixtures have been vigorously agitated by a magnetic stirrer. Experimental points with a high content in glycerol, due to its high viscosity, were stirrer for more than 15 minutes to assurance a sufficient mixture of the compounds at the operating temperature. The temperature was controlled by a thermostatic bath with a precision of $\pm 0.5 \mathrm{~K}$. To determine the mass added of the third component, the mixture was weighed again. The amount of the third component was also determined from the mass change of the syringe before and after titration. The same results were obtained by these two measurements. The cloud point was considered to be a binodal curve point. Samples were collected for density analysis. This way an expression for density as a function of weight fraction for the three components can be obtained. Each experimental point was replicated at least twice.

Tie lines determination. Experiments were carried out in equilibrium cells of $20 \mathrm{~cm}^{3}$. A mixture of sardine oil, glycerol and tertiary alcohol, at a given composition, was prepared directly inside the equilibrium cell by weighing known quantities of each component in an analytical balance (Sartorius Basic, accurate $\pm 0.0001 \mathrm{~g}$ ). The equilibrium temperature was controlled by a thermostatic bath $( \pm 0.5 \mathrm{~K})$. The ternary mixture was then vigorously stirred for at least $3 \mathrm{~h}$ to allow contact between the two liquid phases. After that, the mixture was allowed to stand for at least $24 \mathrm{~h}$ at constant temperature to ensure equilibrium was reached and two transparent liquid phases with a defined interface could be clearly observed. The upper phase was the oil-rich phase and the lower phase was the glycerol-rich phase. Samples of both phases were collected and composition was determined by HT-GC and density measurements. Tie line experiments were replicated twice.

\subsection{Analytical methods}

The sardine oil used in this work was analyzed by gas chromatography to determine the fatty acid profile by the AOAC method (AOAC Official Method 991.39 2000). The fatty acid 
methyl esters were firstly prepared and then analyzed by gas chromatography (GC) in a Hewlett Packard gas chromatograph (6890N Network GC System) equipped with an autosampler (7683B series) and a flame ionization detector (FID). A fused silica capillary column (OmegawaxTM-320, 30m×0.32mm i.d.) was used. Most of the fatty acid methyl esters were identified by comparison of their retention times with those of chromatographic standards (Sigma Chemical Co.). Further details of the gas chromatographic method can be found elsewhere (Rebolleda et al. 2012). Table 1.2 shows the fatty acid composition of the sardine oil. Bandarra et al. (Bandarra et al. 1997) analyzed the seasonal change in lipid composition of sardine oil in terms of fatty acid profile. Although difference can be found among the individual fatty acids, a similar fatty acid profile as the reported in this work (Table 2) is obtained when comparing the total saturated (SFA), monounsaturated (MUFA) and polyunsaturated (PUFA) fatty acids (SFA = $27 \pm 1 ;$ MUFA $=23 \pm 2$ and PUFA $=43 \pm 3$; (Bandarra et al. 1997)). Based on the fatty acid profile obtained in this work, a molecular weight for sardine oil of $879 \mathrm{~g} \cdot \mathrm{mol}-1$ has been estimated.

The free fatty acid (FFA) content of the sardine oil has been determined according to AOCS (AOCS Official Method Ca 5a-40 2009). An automatic titrator Methrom, model Titrando 905 was used. The FFA content for the refined sardine oil was $0.2 \pm 0.1 \%$ expressed as percentage of oleic acid. Due to the low free fatty acid content, the studied systems have been considered as a pseudoternary mixture as it will be explained in section 3.2.

The composition of the tie lines has been determined by using High-Temperature Gas Chromatography (HT-GC). A Hewlett Packard (HP 6890 Series GC System) gas chromatograph equipped with a flame ionization detector (FID), a fused silica capillary column of $30 \mathrm{~m} \times 0.25 \mathrm{~mm}$ i.d. coated with a $0.25 \mathrm{~mm}$ film thickness of $65 \%$ Phenyl Methylpolisiloxane $(65 \mathrm{HT})$ as a stationary phase and Agilent Technologies 7683B Series automatic injector was used. The initial oven temperature was $120^{\circ} \mathrm{C}$ for $2 \mathrm{~min}$, and was then raised to $340{ }^{\circ} \mathrm{C}$ at a rate of $15.0^{\circ} \mathrm{C} \cdot \mathrm{min}^{-1}$. Then it was raised again to $365^{\circ} \mathrm{C}$ at a rate of $1.5^{\circ} \mathrm{C} \cdot \mathrm{min}^{-1}$ and held isothermally for $4 \mathrm{~min}$. The injector temperature was kept at $380^{\circ} \mathrm{C}$, while the detector temperature was $400{ }^{\circ} \mathrm{C}$. Helium $\left(1 \mathrm{~mL} \cdot \mathrm{min}^{-1}\right.$ column constant flow) was used as carrier gas. Split injection mode was used with a ratio of 1:40. 
Table 1.2. Fatty acid composition of the sardine oil and for the two phases (oil and glycerol phases) of a tie line at temperature $\mathrm{T}=303.2 \mathrm{~K}$ for the system glycerol (1) + sardine oil (2) + tert-butanol (3). ${ }^{\text {a }}$

\begin{tabular}{llccc} 
Fatty acid & & Sardine oil, \% & Tie line \\
Myristic & C14:0 & 7.6 & 7.7 & GP, \% \\
Palmitic & C16:0 & 18.1 & 19.1 & 22.1 \\
Palmitoleic & C16:1 & 8.9 & 8.9 & 11.3 \\
Stearic & C18:0 & 3.6 & 3.8 & 2.2 \\
Oleic & C18:1n-9 & 10.0 & 10.1 & 11.7 \\
Vaccenic & C18:1n-7 & 3.8 & 3.9 & 2.2 \\
Linoleic cis (LA) & C18:2n-6 & 2.5 & 2.5 & nd \\
$\alpha$-Linolenic (ALA) & C18:3n-3 & 1.1 & 1.1 & nd \\
Steriadonic & C18:4n-3 & 3.6 & 3.4 & 2.9 \\
Eicosatrienoic & C20:3n-3 & 1.7 & 1.7 & $\mathrm{nd}$ \\
Eicosapentaenoic (EPA) & $\mathrm{C} 20: 5 \mathrm{n}-3$ & 25.9 & 25.0 & 26.3 \\
Docosapentaenoic (DPA) & $\mathrm{C} 22: 5 \mathrm{n}-3$ & 2.7 & 2.7 & $\mathrm{nd}$ \\
Docosahexaenoic (DHA) & $\mathrm{C} 22: 6 \mathrm{n}-3$ & 10.6 & 10.3 & 10.6 \\
\hline
\end{tabular}

GP: glycerol phase; OP: oil phase

${ }^{a}$ Standard uncertainties $u$ are $u$ (percentage $)=0.5$.

Tertiary alcohols and oil have been successfully quantified; however quantification of glycerol was not very reliable due to the bad resolution of the glycerol peak by using this kind of columns. In the last years HT-GC has been proved to be an affective technique to characterize TAGs from different vegetable sources. However, HT-GC could thermally degrade triacylglycerol species that contain polyunsaturated fatty acids, as in fish oils (Lee \& Hastilow 1999). Nevertheless, in this work, the objective is the quantification of sardine oil in terms of total amount of oil and it is not expected to characterize the different TAGs of sardine oil. To quantify total amount of sardine oil a convenient calibration has been performed, as well as for the other components of the mixture. Although degradation of some TAGs species could have been taken place during the HT-GC analysis, this fact would 
be convenient corrected by using the calibration curve. To show the reliability of HT-GC to quantify sardine oil, composition of the three components in the tie line was also determined by density measurements. According to Maduro and Aznar (Maduro \& Aznar 2008), a density calibration curve was obtained from the cloud point determination as a function of the composition of the three components, although the composition of the third component can be obtained by a simple mass balance; therefore, for tie line measurements, density and only one composition, in this work tertiary alcohols composition, must be known to determine the composition of the other components through a density expression of the three components of the mixture and by material balance.

\section{Results and discussion}

\subsection{Experimental data}

The binodal curve and the density data at $303.2 \mathrm{~K}$ and $323.2 \mathrm{~K}$ for two systems: glycerol + sardine oil + tert-butanol and for glycerol + sardine oil + tert-pentanol are presented in Tables 1.3 and 1.4, respectively. In this work, a direct fit of the density data, similar to the approach used in the correlation of the boiling points of ternary mixtures without using binary data suggested by Tamir (Tamir 1981), has been used. The expression is:

$\rho=\sum_{i=1}^{N} w_{i} \rho_{i}+\sum_{i=1}^{N-1} \sum_{j=i+1}^{N} w_{i} w_{j}\left[A_{i j}+B_{i j}\left(w_{i}-w_{j}\right)+C_{i j}\left(w_{i}-w_{j}\right)^{2}+\ldots\right]$

The coefficients of the empirical Eq. 1 were determined by using the Marquardt algorithm.

Table 1.5 lists the values of the adjustable parameters for each system at the two studied temperatures. 
Table 1.3. Experimental (liquid + liquid) equilibrium weight fractions $w$ (binodal curve data) for the system glycerol (1) + sardine oil (2) + tert-butanol (3) at temperature $\mathrm{T}=$ $303.2 \mathrm{~K}$ and $323.2 \mathrm{~K}^{a}$

\begin{tabular}{cccc}
$w_{1}$ & $w_{2}$ & $w_{3}$ & $\rho / k g \cdot m^{-3}$ \\
\multicolumn{4}{c}{$\mathrm{T}=303.2 \mathrm{~K}$} \\
0.0016 & 0.9984 & 0.0000 & 923.48 \\
0.0069 & 0.8648 & 0.1283 & 903.29 \\
0.0256 & 0.7163 & 0.2581 & 884.94 \\
0.0365 & 0.6056 & 0.3579 & 870.28 \\
0.0521 & 0.5144 & 0.4335 & 864.06 \\
0.0586 & 0.4809 & 0.4605 & 861.47 \\
0.0717 & 0.4039 & 0.5244 & 854.94 \\
0.1044 & 0.2851 & 0.6105 & 849.67 \\
0.1271 & 0.1953 & 0.6776 & 845.95 \\
0.1649 & 0.1149 & 0.7202 & 851.99 \\
0.2076 & 0.0689 & 0.7235 & 858.72 \\
0.3122 & 0.0153 & 0.6725 & 887.25 \\
0.3330 & 0.0168 & 0.6502 & 903.10 \\
0.4244 & 0.0039 & 0.5717 & 934.64 \\
0.7361 & 0.0006 & 0.2633 & 1092.49 \\
0.9967 & 0.0000 & 0.0033 & 1244.91
\end{tabular}

\begin{tabular}{lccc}
\multicolumn{3}{c}{$T=323.2 \mathrm{~K}$} \\
0.0043 & 0.9769 & 0.0188 & \\
0.0100 & 0.9048 & 0.0852 & 806.54 \\
0.0302 & 0.6840 & 0.2858 & 867.25 \\
0.0378 & 0.6298 & 0.3324 & 862.68 \\
0.0471 & 0.5743 & 0.3786 & 856.39 \\
0.0612 & 0.5293 & 0.4095 & 853.12 \\
0.1037 & 0.3825 & 0.5138 & 843.60 \\
0.1480 & 0.2437 & 0.6083 & 841.47 \\
0.1844 & 0.1713 & 0.6443 & 846.82 \\
0.2542 & 0.0723 & 0.6735 & 855.92 \\
0.3361 & 0.0285 & 0.6354 & 888.24 \\
0.4725 & 0.0047 & 0.5228 & 942.45 \\
0.4995 & 0.0042 & 0.4963 & 954.57 \\
0.8011 & 0.0019 & 0.1970 & 1095.16 \\
\hline
\end{tabular}

${ }^{\mathrm{a}}$ Standard uncertainties $u$ are $u(T)=0.5 \mathrm{~K}, u(x)=0.0005, u(\rho)=0.05$. 
Table 1.4. Experimental (liquid + liquid) equilibrium weight fractions $w$ (binodal curve data) for the system glycerol (1) + sardine oil (2) + tert-pentanol (3) at $303.2 \mathrm{~K}$ and $323.2 \mathrm{~K}^{a}$

\begin{tabular}{|c|c|c|c|}
\hline$w_{1}$ & $w_{2}$ & $w_{3}$ & $\rho / \mathrm{kg} \cdot \mathrm{m}^{-3}$ \\
\hline \multicolumn{4}{|c|}{$\mathrm{T}=303.2 \mathrm{~K}$} \\
\hline 0.0027 & 0.9056 & 0.0917 & 909.61 \\
\hline 0.0025 & 0.8665 & 0.1310 & 905.46 \\
\hline 0.0045 & 0.8247 & 0.1708 & 900.43 \\
\hline 0.0021 & 0.7361 & 0.2618 & 893.52 \\
\hline 0.0159 & 0.6315 & 0.3526 & 885.58 \\
\hline 0.0523 & 0.5175 & 0.4302 & 881.25 \\
\hline 0.0683 & 0.4783 & 0.4534 & 879.86 \\
\hline 0.1105 & 0.3756 & 0.5139 & 881.20 \\
\hline 0.1635 & 0.2539 & 0.5826 & 888.48 \\
\hline 0.2192 & 0.1589 & 0.6219 & 893.66 \\
\hline 0.3038 & 0.0640 & 0.6322 & 914.05 \\
\hline 0.4170 & 0.0199 & 0.5631 & 953.34 \\
\hline 0.4700 & 0.0101 & 0.5199 & 973.88 \\
\hline 0.6544 & 0.0033 & 0.3423 & 1058.99 \\
\hline \multicolumn{4}{|c|}{$\mathrm{T}=323.2 \mathrm{~K}$} \\
\hline 0.0051 & 0.8414 & 0.1535 & 886.88 \\
\hline 0.0066 & 0.8074 & 0.1860 & 883.61 \\
\hline 0.0223 & 0.6274 & 0.3503 & 868.91 \\
\hline 0.0854 & 0.4406 & 0.4740 & 865.18 \\
\hline 0.1561 & 0.2969 & 0.5470 & 869.77 \\
\hline 0.1843 & 0.2490 & 0.5667 & 874.89 \\
\hline 0.2155 & 0.2005 & 0.5840 & 881.34 \\
\hline 0.2817 & 0.1179 & 0.6004 & 893.46 \\
\hline 0.3124 & 0.0885 & 0.5991 & 902.49 \\
\hline 0.4948 & 0.0147 & 0.4905 & 969.68 \\
\hline 0.7151 & 0.0043 & 0.2806 & 1076.68 \\
\hline 0.8462 & 0.0017 & 0.1521 & 1143.25 \\
\hline
\end{tabular}

${ }^{\mathrm{a}}$ Standard uncertainties $\mathrm{u}$ are $\mathrm{u}(\mathrm{T})=0.5 \mathrm{~K}, \mathrm{u}(\mathrm{x})=0.0005, \mathrm{u}(\rho)=0.05$.

Figures 1.1 and 1.2 show the binodal curves for the systems studied in this work. The large two-phase region shows high immiscibility between glycerol and sardine oil even in the presence of the tertiary alcohols. The information provided by the binodal curve is necessary to optimize the amount of solvent used to create a homogeneous system 
containing the reactants, glycerol and sardine oil, taking into account the corresponding reactant molar ratio of the glycerolysis system. It can be observed that, in the temperature range covered in this work (303.2-323.2 K), the effect of temperature is not significant on decreasing the biphasic region. This behaviour has been also observed for ternary mixtures involving biodiesel and glycerol together with different alcohols (França et al. 2009; Mesquita et al. 2012; Oliveira et al. 2011). This means that the reaction temperature of the glycerolysis system can be determined in terms of kinetic parameters rather than in order to increase the mutual solubility of reactants. Additionally, it can be observed that at a given temperature, the miscibility region is bigger for tert-pentanol than for tert-butanol (see Figures 1.1 and 1.2).

Table 1.5. Parameters of equation 1

\section{System} $\mathrm{T} / \mathrm{K}$

Parameters

Glycerol (1) + sardine oil (2) + tert-butanol (3)

Glycerol (1) + sardine oil (2) + tert-pentanol (3)
303.

$\begin{array}{lll}\mathrm{A}_{12}=562.24 & \mathrm{~B}_{12}=606.77 & \\ \mathrm{~A}_{13}=-181.51 & \mathrm{~B}_{13}=8.20 & 0.9997 \\ \mathrm{~A}_{23}=-77.64 & \mathrm{~B}_{23}=70.33 & \\ \mathrm{~A}_{12}=-828.77 & \mathrm{~B}_{12}=-989.29 & \\ \mathrm{~A}_{13}=-168.48 & \mathrm{~B}_{13}=-199.14 & 0.9986 \\ \mathrm{~A}_{23}=19.24 & \mathrm{~B}_{23}=-68.10 & \\ \mathrm{~A}_{12}=-370.85 & \mathrm{~B}_{12}=334.08 & \\ \mathrm{~A}_{13}=-163.54 & \mathrm{~B}_{13}=-11.81 & 0.9998 \\ \mathrm{~A}_{23}=57.31 & \mathrm{~B}_{23}=-85.09 & \\ \mathrm{~A}_{12}=-386.53 & \mathrm{~B}_{12}=37.75 & \\ \mathrm{~A}_{13}=-160.66 & \mathrm{~B}_{13}=-36.12 & 0.9998 \\ \mathrm{~A}_{23}=39.15 & \mathrm{~B}_{23}=-78.65 & \end{array}$




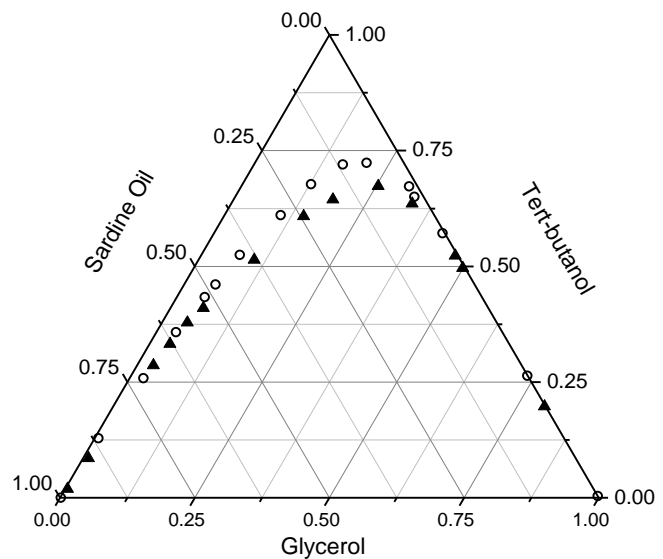

Figure 1.1. Phase diagram of the system glycerol + sardine oil + tert-butanol (o $303.2 \mathrm{~K}$; $323.2 \mathrm{~K})$

The tie line data for the system glycerol + sardine oil + tert-butanol (or tert-pentanol) are presented in Tables $\mathbf{1 . 6}$ and $\mathbf{1 . 7}$ respectively. Composition of the tie lines was determined by HT-GC as well as by density calibration using the tertiary alcohols as key components. By using the parameters from Table 1.5, composition of sardine oil and glycerol could be also determined. Composition of sardine oil and glycerol calculated by density calibration and by HT-GC were similar. Deviations between both methods are lower than $2 \%$ for the major compound in the equilibrium phases. However relative deviations found for the minor compound in the equilibrium phases are noticeably higher. From the shape of the binodal curves for both systems it can be observed that the amount of oil and glycerol in the glycerol and oil phases respectively is very small. This fact can be clearly observed in the composition of oil in the glycerol-rich phase, since for most experimental tie lines, oil content in the glycerol-rich phase is lower than $1 \%$. Therefore, relative deviations between the two analytical methods for oil and glycerol composition in the glycerol-rich phase and in the oil-rich phase, respectively, are higher. Relative deviation can reach values up to $50 \%$, or even higher, specially for oil content in the glycerol-rich phase. Values reported in Tables 6 and 7 correspond to the values obtained by HT-CG analysis. 


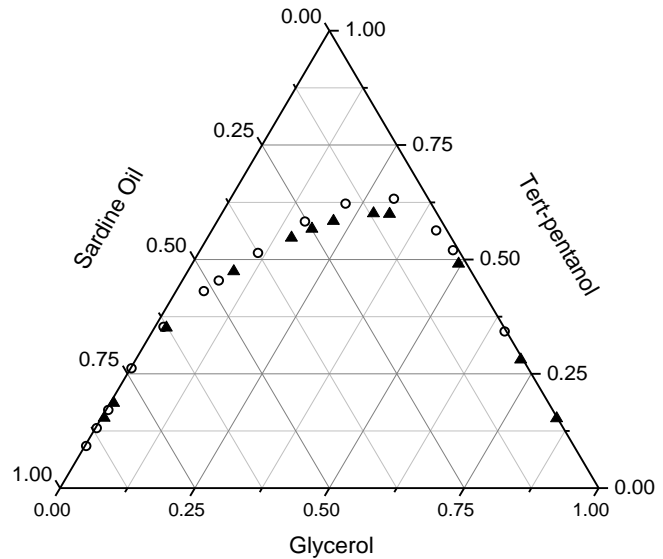

Figure 1.2. Phase diagram of the system glycerol + sardine oil + tert-pentanol (o $303.2 \mathrm{~K}$; $323.2 \mathrm{~K})$

Tie lines have been plotted in Figures 1.3-1.4 and 1.5-1.6 for the systems glycerol + sardine oil + tert-butanol and glycerol + sardine oil + tert-pentanol respectively. Tie lines show that for the tert-butanol system, the glycerol phase is richer in tert-butanol than the oil phase. However for the ternary system with tert-pentanol, the oil phase is richer in the tertiary alcohol, although in this case the slope of the tie line is lower than in the system with tert-butanol. In the literature, it has been also shown a different thermodynamic behaviour of tert-butanol in relation to other 2-methyl-2-alcohols (González et al. 1996). González et al. (González et al. 1996), in the extension of DISQUAC equation to different mixtures with tert-alcohols, concluded that the same quasichemical coefficients characteristic of each tert-alcohols series could be used, excluding tert-butanol. The different behaviour observed for tert-butanol and tert-pentanol could be related with the corresponding values of the dielectric constant for both tert-acohols (Etert-butanol, $25^{\circ} \mathrm{C}=$ 12.47; ctert-pentanol, $25^{\circ} \mathrm{C}=5.78$ ) (Riddick et al. 1986). Dielectric constant of a compound is an index of its polarity. Based on the different values of the dielectric constant for the two tert-alcohols studied in this work, it seems reasonable that tie lines for the tertbutanol system present positive slope since glycerol present a high value of the dielectric constant (cglycerol, $25^{\circ} \mathrm{C}=42.5$ ), especially when comparing this value with the dielectric constant of fish oil $\left(\varepsilon_{\text {fish oil, }} 25^{\circ} \mathrm{C}=2.76\right.$ ) (Americas 2012). The differences observed in the 
$\log \mathrm{P}$ for tert-butanol and tert-pentanol $(\log \mathrm{P}$ tert-butanol $=0.35$ and $\log \mathrm{P}$ tert-pentanol $=$ 0.89) could also support the different slope observed in the tie lines (Damstrup, Abildskov, et al. 2006).

Table 1.6. Experimental (liquid + liquid) equilibrium data for the system glycerol (1) + sardine oil (2) + tert-butanol (3) for weight fractions $\mathrm{w}$ at temperature $\mathrm{T}=303.2 \mathrm{~K}$ and $\mathrm{T}=$ $323.2 \mathrm{~K}^{\mathrm{a}}{ }^{\mathrm{a}}$

\begin{tabular}{|c|c|c|c|c|c|c|c|c|}
\hline \multicolumn{3}{|c|}{ Overall composition } & \multicolumn{3}{|c|}{ Glycerol-rich phase } & \multicolumn{3}{|c|}{ Oil-rich phase } \\
\hline $\mathrm{w}_{1}$ & $w_{2}$ & $w_{3}$ & $w_{1}$ & $w_{2}$ & $w_{3}$ & $w_{1}$ & $w_{2}$ & $w_{3}$ \\
\hline \multicolumn{9}{|c|}{$\mathrm{T}=303.2 \mathrm{~K}$} \\
\hline 0.2503 & 0.2504 & 0.4993 & 0.3470 & 0.0397 & 0.6133 & 0.0125 & 0.7508 & 0.2367 \\
\hline 0.3003 & 0.2998 & 0.3999 & 0.4412 & 0.0223 & 0.5365 & 0.0169 & 0.7930 & 0.1901 \\
\hline 0.3504 & 0.3499 & 0.2997 & 0.5690 & 0.0191 & 0.4119 & 0.0161 & 0.8243 & 0.1596 \\
\hline 0.4002 & 0.3999 & 0.1999 & 0.7280 & 0.0020 & 0.2700 & 0.0075 & 0.8487 & 0.1438 \\
\hline 0.4485 & 0.4500 & 0.1015 & 0.8594 & 0.0191 & 0.1215 & 0.0055 & 0.8744 & 0.1201 \\
\hline \multicolumn{9}{|c|}{$\mathrm{T}=323.2 \mathrm{~K}$} \\
\hline 0.2502 & 0.2504 & 0.4994 & 0.3673 & 0.0238 & 0.6089 & 0.0245 & 0.6994 & 0.2761 \\
\hline 0.2998 & 0.3003 & 0.3999 & 0.4843 & 0.0095 & 0.5062 & 0.0185 & 0.7323 & 0.2492 \\
\hline 0.3499 & 0.3501 & 0.3000 & 0.6070 & 0.0076 & 0.3854 & 0.0106 & 0.7539 & 0.2355 \\
\hline 0.3984 & 0.3963 & 0.2053 & 0.7491 & 0.0066 & 0.2443 & 0.0062 & 0.8299 & 0.1639 \\
\hline 0.4500 & 0.4501 & 0.0999 & 0.8944 & 0.0004 & 0.1052 & 0.0051 & 0.9044 & 0.0905 \\
\hline
\end{tabular}

${ }^{\mathrm{a}}$ Standard uncertainties $u$ are $u(T)=0.5 \mathrm{~K}, u(x)=0.0005$.

The reliability of the tie-line data were tested by Othmer-Tobias equation (Othmer \& Tobias 1942):

$$
\ln \left(\frac{1-\mathrm{w}_{2}^{\mathrm{OP}}}{\mathrm{w}_{2}^{\mathrm{OP}}}\right)=\mathrm{A}+\mathrm{B} \ln \left(\frac{1-\mathrm{w}_{1}^{\mathrm{GP}}}{\mathrm{w}_{1}^{\mathrm{GP}}}\right)
$$


where $w_{2}^{O P}$ is the mass fraction of sardine oil in the oil-rich phase and $w_{1}^{G P}$ is the mass fraction of glycerol in the glycerol-rich phase. The Othmer-Tobias plot for the studied systems is shown in Figure 1.7. The linearity of the plot indicates a good degree of consistency of the experimental data.

Table 1.7. Experimental (liquid + liquid) equilibrium data for the system glycerol (1) + sardine oil (2) + tert-pentanol (3) for weight fractions $\mathrm{w}$ at temperature $\mathrm{T}=303.2 \mathrm{~K}$ and $\mathrm{T}=$ $323.2 \mathrm{~K}^{\mathrm{a}}{ }^{\mathrm{a}}$

\begin{tabular}{|c|c|c|c|c|c|c|c|c|}
\hline \multicolumn{3}{|c|}{ Overall composition } & \multicolumn{3}{|c|}{ Glycerol-rich phase } & \multicolumn{3}{|c|}{ Oil-rich phase } \\
\hline $\mathrm{w}_{1}$ & $w_{2}$ & $w_{3}$ & $\mathrm{w}_{1}$ & $w_{2}$ & $w_{3}$ & $\mathrm{w}_{1}$ & $w_{2}$ & $w_{3}$ \\
\hline \multicolumn{9}{|c|}{$\mathrm{T}=303.2 \mathrm{~K}$} \\
\hline 0.3506 & 0.3502 & 0.2992 & 0.7451 & 0.0027 & 0.2522 & 0.0303 & 0.6238 & 0.3459 \\
\hline 0.4002 & 0.3907 & 0.2091 & 0.8470 & 0.0121 & 0.1409 & 0.0108 & 0.7155 & 0.2737 \\
\hline 0.4199 & 0.4203 & 0.1598 & 0.8776 & 0.0158 & 0.1066 & 0.0123 & 0.7729 & 0.2148 \\
\hline 0.4492 & 0.4433 & 0.1075 & 0.9182 & 0.0085 & 0.0733 & 0.0074 & 0.8476 & 0.1450 \\
\hline \multicolumn{9}{|c|}{$\mathrm{T}=323.2 \mathrm{~K}$} \\
\hline 0.3505 & 0.3501 & 0.2994 & 0.7922 & 0.0007 & 0.2071 & 0.0389 & 0.6117 & 0.3494 \\
\hline 0.3797 & 0.3803 & 0.2400 & 0.8545 & 0.0048 & 0.1407 & 0.0257 & 0.6622 & 0.3121 \\
\hline 0.3994 & 0.4003 & 0.2003 & 0.8914 & 0.0054 & 0.1032 & 0.0105 & 0.7110 & 0.2785 \\
\hline 0.4492 & 0.4486 & 0.1022 & 0.9411 & 0.0015 & 0.0574 & 0.0121 & 0.8317 & 0.1562 \\
\hline
\end{tabular}

${ }^{\mathrm{a}}$ Standard uncertainties $u$ are $u(T)=0.5 \mathrm{~K}, u(x)=0.0005$. 


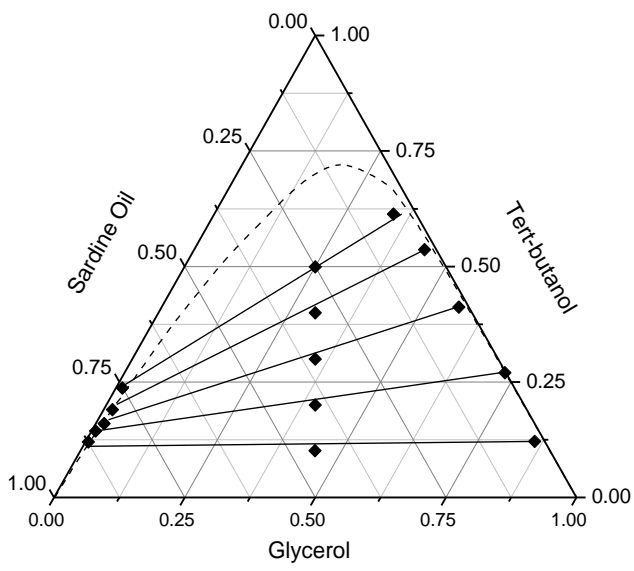

Figure 1.3. Liquid-liquid equilibrium for the system glycerol + sardine oil + tert-butanol at 303.2 K: ---, binodal curve; tie lines; - NRTL $(\alpha=0.5)$.

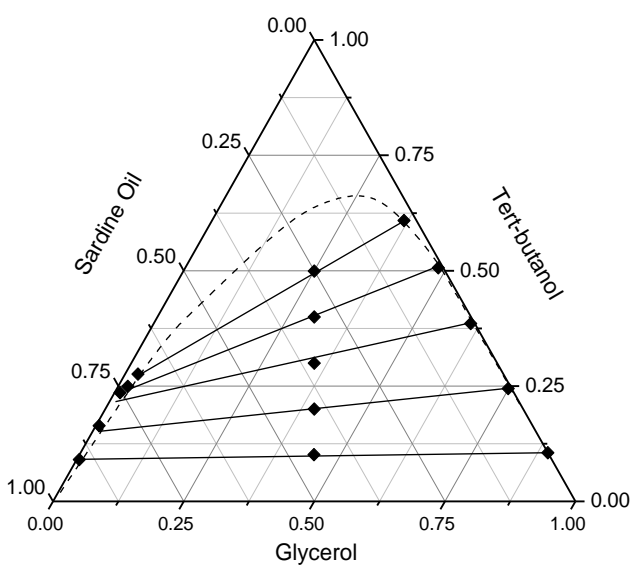

Figure 1.4. Liquid-liquid equilibrium for the system glycerol + sardine oil + tert-butanol at 323.2 K: ---, binodal curve; tie lines; - NRTL $(\alpha=0.5)$. 


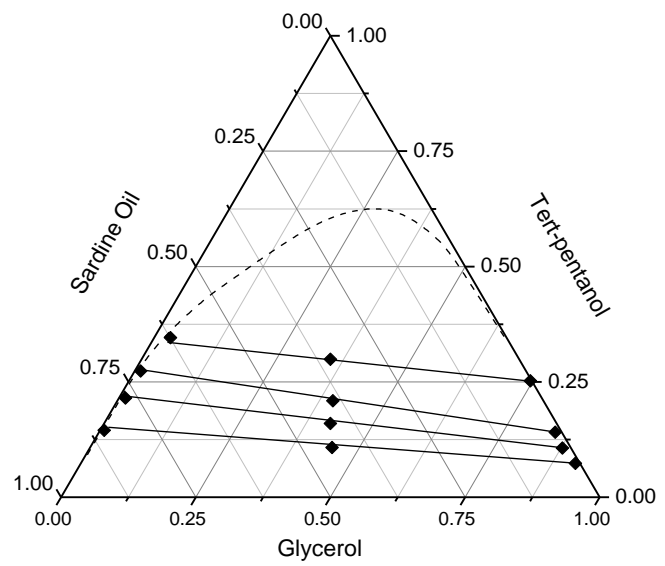

Figure 1.5. Liquid-liquid equilibrium for the system glycerol + sardine oil + tert-pentanol at 303.2 K: ---, binodal curve; tie lines; - NRTL $(\alpha=0.4)$.

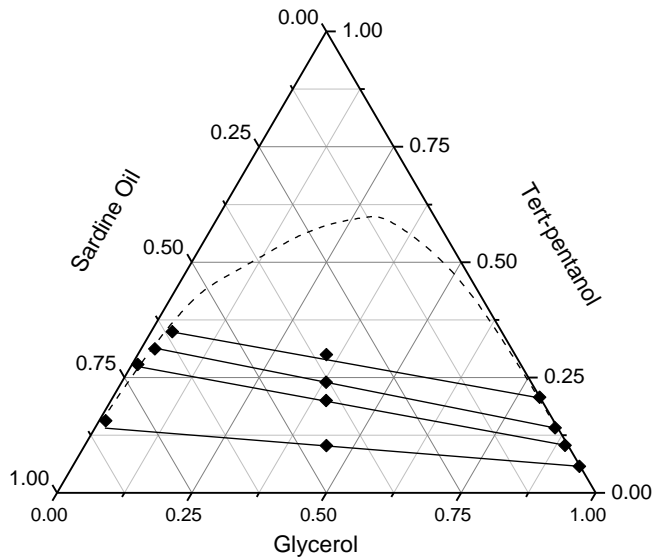

Figure 1.6. Liquid-liquid equilibrium for the system glycerol + sardine oil + tert-pentanol at $323.2 \mathrm{~K}:---$, binodal curve; tie lines; - NRTL $(\alpha=0.4)$. 


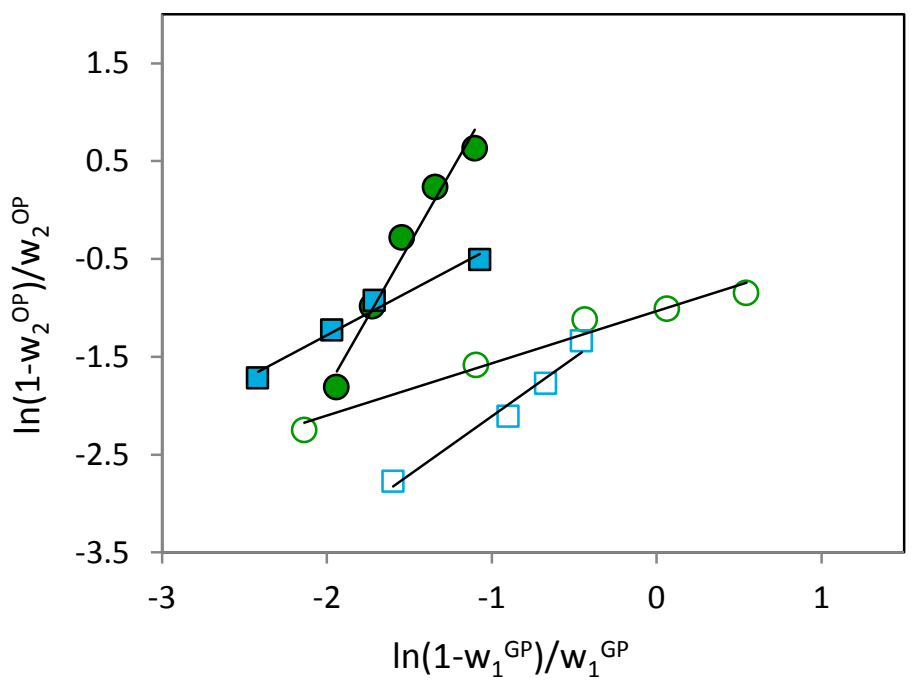

Figure 1.7. Othmer-Tobias plot for the system glycerol (1) + sardine oil (2) + tert-butanol (3) at $303.2 \mathrm{~K}\left(\bullet, \mathrm{r}^{2}=0.9683\right)$ and $323.2 \mathrm{~K}\left(0, \mathrm{r}^{2}=0.9704\right)$ and for the system glycerol (1) + sardine oil (2) + tert-pentanol (3) at $303.2 \mathrm{~K}\left(\square, \mathrm{r}^{2}=0.9775\right)$ and $323.2 \mathrm{~K}\left(\square, \mathrm{r}^{2}=0.9701\right)$.

\subsection{Data correlation}

In the correlation of the liquid-liquid equilibrium data, the sardine oil has been treated as a single compound. This assumption implies that the different triacylglycerols present in the sardine oil behave in a similar way in the liquid-liquid system. Table 1.1 shows, along with the fatty acid composition of the sardine oil, the fatty acid composition of the corresponding oil-rich phase and glycerol-rich phase for a tie line obtained at $303.2 \mathrm{~K}$ in the system glycerol (1) + sardine oil (2) + tert-butanol (3). Fatty acid distribution in the oilrich phase is similar to the fatty acid profile of sardine oil. Relative deviations range between 1.0 \% for oleic acid and $5.6 \%$ for palmitoleic acid. However, deviations found in the fatty acid profile of the glycerol-rich phase are higher due to low content of oil in the glycerol rich phase. Quantification of fatty acids depends on the detection limit of the detector in the GC. In fact, minority fatty acids in sardine oil, such as $\alpha$-linolenic or eicosatrienoic acids, could not be properly detected in the glycerol-rich phase (see Table 6 and 7). Anyway, it can be assumed a similar distribution of the fatty acids in both phases. Similar results were obtained for the other tie lines studied in this work. The same 
approach has been used in the literature when dealing with different types of oil (Rodrigues et al. 2004). This result supports the previous assumption of considering the sardine oil as a single compound.

Experimental tie line data were correlated to the NRTL model. Mass fraction was used as composition unit, instead of mole fraction due to the large difference in molar mass of the components of the system. This is usual in most of the literature dealing with systems including different kinds of vegetable oils (Lanza et al. 2009; Lanza et al. 2008; Rodrigues et al. 2004).This way, the isoactivity criterion of phase equilibrium can be expressed in mass fraction units as follows:

$\left(\gamma_{i}^{w} w_{i}\right)^{G P}=\left(\gamma_{i}^{w} w_{i}\right)^{O P}$

The mass fraction-scale activity coefficient $\gamma_{i}^{w}$ must be related to the NRTL activity coefficient $\gamma_{i}$ by the following equation (Lanza et al. 2008; Rodrigues et al. 2005; Da Silva et al. 2011):

$$
\gamma_{i}^{w}=\frac{\gamma_{i}}{M_{i} \sum_{j}^{n}\left(\frac{w_{j}}{M_{j}}\right)}
$$

where $M_{i}$ and $M_{j}$ are the molecular weight of component $i$ and $j$ respectively and $w_{j}$ is the mass fraction of component j respectively.

Rodrigues et al. and Gonçalves et al. (Gonçalves et al. 2002; Rodrigues et al. 2004) showed an expression for the activity coefficient, $\gamma_{i}$, for the NRTL model using mass fractions as unity of concentration:

$\ln \gamma_{\mathrm{i}}=\frac{\sum_{\mathrm{j}=1}^{\mathrm{K}} \tau_{\mathrm{ji}} \mathrm{G}_{\mathrm{ji}} \mathrm{w}_{\mathrm{j}} / \mathrm{M}_{\mathrm{j}}}{\sum_{\mathrm{j}=1}^{\mathrm{K}} \mathrm{G}_{\mathrm{ji}} \mathrm{w}_{\mathrm{j}} / \mathrm{M}_{\mathrm{j}}}+\sum_{\mathrm{j}=1}^{\mathrm{K}}\left[\frac{\mathrm{w}_{\mathrm{j}} \mathrm{G}_{\mathrm{ji}}}{\mathrm{M}_{\mathrm{j}} \sum_{\mathrm{l}=1}^{\mathrm{n}} \mathrm{G}_{\mathrm{lj}} \mathrm{w}_{\mathrm{l}} / \mathrm{M}_{\mathrm{l}}} \times\left(\tau_{\mathrm{ij}}-\frac{\sum_{\mathrm{l}=1}^{\mathrm{K}} \tau_{\mathrm{lj}} \mathrm{G}_{\mathrm{lj}} \mathrm{w}_{\mathrm{l}} / \mathrm{M}_{\mathrm{l}}}{\sum_{\mathrm{l}=1}^{\mathrm{K}} \mathrm{G}_{\mathrm{lj}} \mathrm{w}_{\mathrm{l}} / \mathrm{M}_{\mathrm{l}}}\right)\right]$ 
where

$\mathrm{G}_{\mathrm{ij}}=\exp \left(-\alpha_{\mathrm{ij}} \tau_{\mathrm{ij}}\right)$

$\tau_{\mathrm{ij}}=\frac{\mathrm{A}_{\mathrm{ij}}}{\mathrm{T}}$

$\alpha_{\mathrm{ij}}=\alpha_{\mathrm{ji}}$

$\mathrm{A}_{\mathrm{ij}}$ and $\alpha_{\mathrm{ij}}$ are parameters of the NRTL model, $\mathrm{w}$ is the mass fraction, $\mathrm{M}$ is the molecular weight and $\mathrm{T}$ is the equilibrium temperature. The NRTL model has three parameters for each binary mixture: $A_{i j}$ and $A_{j i}$ represent the interaction energy between compounds $i$ and $\mathrm{j}$ and $\alpha_{\mathrm{ij}}$ is a nonrandomness parameter derived from the local composition assumption. The parameter estimation was based on the minimization of the following objective function by using the Simplex Nelder Mead method:

$\mathrm{OF}=\sum_{\mathrm{k}}^{\mathrm{D}} \sum_{\mathrm{j}}^{\mathrm{M}} \sum_{\mathrm{i}}^{\mathrm{N}-1}\left[\left(\mathrm{w}_{\mathrm{ijk}}^{\mathrm{GP}, \exp }-\mathrm{w}_{\mathrm{ijk}}^{\mathrm{GP}, \text { calc }}\right)^{2}+\left(\mathrm{w}_{\mathrm{ijk}}^{\mathrm{OP}, \exp }-\mathrm{w}_{\mathrm{ijk}}^{\mathrm{OP}, \text { calc }}\right)^{2}\right]$

where $D$ is the number of data sets (number of studied temperatures for each system), $M$ is the number of tie-lines in each data set, $\mathrm{N}$ is the number of components, the superscripts GP and OP refer to glycerol-rich phase and oil-rich phase respectively, and the superscripts exp and calc refer to the experimental and calculated values of the liquidphase concentration.

In the minimization procedure to calculate the equilibrium concentrations a procedure similar to the proposed by Reyes-Labarta et al. (Reyes-Labarta et al. 2009) has been followed. The concentrations that satisfy the isoactivity criterion and the mass balances in each phase are calculated by the Newton-Raphson method by fixing pressure, temperature and composition of one of the components in one phase.

A constant value for the NRTL non-randomness parameter, $\alpha_{i j}$, has been used in the fitting procedure reducing the number of adjustable parameters to just two for each pair of 
compounds. $\alpha_{\mathrm{ij}}$ was set to different values between 0.1 and 0.5 . The best results were achieved for the values presented in Table $\mathbf{1 . 8}$

Table 1.8. NRTL parameters for the systems: glycerol (1) + sardine oil (2) + tert-butanol (3) and for the glycerol (1) +sardine oil (2) + tert-pentanol (3)

\begin{tabular}{|c|c|c|c|c|}
\hline Pair & $A_{i j} / K$ & $A_{\mathrm{ji}} / K$ & $\alpha_{i j}$ & RMS \\
\hline \multicolumn{5}{|c|}{ System: glycerol (1) + sardine oil (2) + tert-butanol (3) } \\
\hline 12 & 2781.9 & 1365.1 & 0.5 & \multirow{3}{*}{0.58} \\
\hline 13 & 620.6 & 941.1 & 0.5 & \\
\hline 23 & -155.6 & 1649.8 & 0.5 & \\
\hline 12 & 5382.9 & 3825.9 & 0.309 & \multirow{3}{*}{0.48} \\
\hline 13 & 720.0 & 1045.3 & 0.429 & \\
\hline 23 & 614.5 & 1506.2 & 0.501 & \\
\hline \multicolumn{5}{|c|}{ System: glycerol (1) + sardine oil (2) + tert-pentanol (3) } \\
\hline 12 & 4219.8 & 6102.6 & 0.4 & \multirow{3}{*}{0.48} \\
\hline 13 & 840.1 & 1010.3 & 0.4 & \\
\hline 23 & 48.1 & 1744.1 & 0.4 & \\
\hline 12 & 5012.5 & 2523.4 & 0.362 & \multirow{3}{*}{0.31} \\
\hline 13 & 841.1 & 964.56 & 0.408 & \\
\hline 23 & 439.4 & 1626.4 & 0.454 & \\
\hline
\end{tabular}

Figures 1.3-1.6 show the experimental liquid-liquid equilibrium data and the tie lines calculated by using the NRTL model with the parameters listed in Table $\mathbf{1 . 8}$ for the systems studied in this work. As it can be observed, the NRTL model was able to accurately describe the phase behavior. Comparison between experimental and calculated composition of each component in each of the two phases were made through root mean-square (RMS) deviation, given by the following expression (Gonçalves et al. 2002) (see Table 1.8). 
$\delta \mathrm{w}=100 \sqrt{\frac{\sum_{\mathrm{i}}^{\mathrm{M}} \sum_{\mathrm{j}}^{\mathrm{N}}\left(\mathrm{w}_{\mathrm{ijk}}^{\mathrm{GP}, \exp }-\mathrm{w}_{\mathrm{ijk}}^{\mathrm{GP}, \text { calc }}\right)^{2}+\left(\mathrm{w}_{\mathrm{ijk}}^{\mathrm{OP}, \exp }-\mathrm{w}_{\mathrm{ijk}}^{\mathrm{OP}, \text { calc }}\right)^{2}}{2 \mathrm{MN}}}$

Additionally, in the fitting procedure, $\alpha_{i j}$ was considered as an optimization parameter for each pair of substances (see Table 1.8). RMS was reduced from 0.58 to 0.48 and from 0.48 to 0.31 for systems with tert-butanol and tert-pentanol, respectively.

\section{Conclusions}

Liquid-liquid equilibrium for systems containing sardine oil, glycerol and tertiary alcohol as organic solvents has been studied. Specifically, both binodal and tie-lines were determined for the following ternary systems: glycerol + sardine oil + tert-butanol and glycerol + sardine oil + tert-pentanol at two different temperatures, $303.2 \mathrm{~K}$ and $323.2 \mathrm{~K}$.

The biphasic region seems not to be significantly reduced by increasing temperate in the range covered in this work $(303.2 \mathrm{~K}-323.2 \mathrm{~K})$. The miscibility region is bigger for tertpentanol than for tert-butanol. The experimental data presented in this work are of interest in the correct design of a glycerolysis system to create a homogenous reaction system.

The reliability of the tie-lines was verified by applying the Othmer-Tobias correlation. NRTL activity coefficient model was able to describe the liquid-liquid equilibrium for the two studied systems showing a root mean squared deviation lower than $1 \%$. 



\section{CHAPTER 2}

\section{Glycerolysis of sardine oil catalyzed by a water dependent lipase in different tert- alcohols as reaction medium}

Á. García Solaesa, M.T. Sanz, R. Melgosa, S.L. Bucio and S. Beltrán (2015).

"Glycerolysis of sardine oil catalyzed by a water dependent lipase in different tert-alcohols as reaction medium"

Grasas y aceites, 66 (4): e102 



\section{Capítulo 2}

\section{Glicerolisis de aceite de sardina catalizada por una lipasa dependiente de agua en diferentes tert-alcoholes como medio de reacción}

\section{Resumen}

En este capítulo se ha estudiado la producción de monoglicéridos (MAG), ricos en ácidos grasos poliinsaturados (AGPI), mediante glicerolisis enzimática de aceite de sardina. La reacción se ha llevado a cabo en dos tert-alcoholes, tert-pentanol y tert-butanol, para conseguir de esta forma un sistema homogéneo. La cantidad de disolvente añadida al medio se ha calculado en base al equilibrio líquido-líquido entre los componentes del sistema estudiado en el capítulo 1 de esta tesis. La lipasa empleada como biocatalizador ha sido la enzima inmovilizada Lipozyme RM IM de Rhizomucor miehei, una lipasa dependiente de agua. Se ha estudiado el efecto de distintos parámetros cinéticos, así como de la cantidad de agua añadida al medio de reacciónen la producción de MAG. De los resultados obtenidos, se puede concluir que, para una relación molar inicial de reactantes de 3:1 (glicerol:aceite de sardina), un $12 \%$ de agua en base al glicerol y un $10 \%$ de lipasa, en base a los reactantes; se puede llegar a conseguir un rendimiento en MAG alrededor del $70 \%$, con casi un $28 \%$ de AGPI, aunque con un contenido en ácidos grasos libres de alrededor del $18 \%$. 



\section{Abstract}

The production of monoacylglycerol rich in polyunsaturated fatty acids (PUFA) via enzymatic glycerolysis of sardine oil in a homogeneous system was evaluated. Reactions were conducted in two different tert-alcohols. Based on phase equilibrium data, the amount of solvent added to create a homogeneous system has been calculated and optimized. The immobilized lipase used in this work was Lipozyme RM IM from : Rhizomucor miehei, a water dependent lipase. The amount of water added as well as other reaction parameters were studied to evaluate the optimum conditions for monoacylglycerol obtention. An initial reactant mole ratio glycerol to sardine oil 3:1, 12 wt $\%$ of water based on glycerol content and $10 \mathrm{wt} \%$ of lipase loading (based on reactants weight), achieved a MAG yield around $70 \%$, containing nearly 28 wt\% of PUFA, with low free fatty acid content (lower than $18 \mathrm{wt} \%$ ).

- - - - - - - - - - - - - - - - - - - - - il

\section{Introduction}

Marine raw materials are rich sources of omega-3 PUFA, especially eicosapentaenoic acid (EPA) and docosahexaenoic acid (DHA). Health benefits of omega-3 PUFA have been demonstrated (Nichols et al. 2014; Sidhu 2003). Fish oil can be extracted from the whole fish or from its byproducts as skin or viscera. The use of these byproducts is attractive from an economical point of view. Fish oil is a valuable ingredient in its natural form or as different lipid derivatives. Among these compounds, monoacylglycerols (MAG) and diacylglycerols (DAG) have found extensive applications in food and pharmaceuticals products (Blanco et al. 2007; Hernandez 2014; Kim \& Mendis 2006). MAG are non-ionic amphiphilic molecules with excellent emulsifying properties. Commercial food-grade MAG are usually manufactured by chemical glycerolysis of fats and oils. In these reactions high temperature $\left(220-250{ }^{\circ} \mathrm{C}\right)$ and inorganic alkaline catalysts are used. These chemical and physical processes are not suitable for heat-sensitive oils and fats rich in polyunsaturated fatty acids such as fish oils. Due to the disadvantages of conventional glycerolysis, the enzymatic process is an attractive alternative method for polyunsaturated MAG production because the reaction can be carried out under mild conditions (Bornscheuer 1995). 
The overall glycerolysis reaction is given by Equation 1 (Feltes et al. 2013). This reaction takes place in two consecutive steps. In the first step (Eq. 2), the transfer of a fatty acid (FA) from triacylglycerols (TAG) to glycerol gives one molecule of MAG and one molecule of DAG. In the second step (Eq. 3), two molecules of MAG are formed by the transfer of a FA from DAG to glycerol:

$\begin{array}{llll}\mathrm{TAG}+2 \mathrm{GLY} & \rightleftarrows & 3 \mathrm{MAG} & {[1]} \\ \mathrm{TAG}+\mathrm{GLY} & \rightleftarrows & \mathrm{MAG}+\mathrm{DAG} \\ \mathrm{DAG}+\mathrm{GLY} & \rightleftarrows & \text { [2] }\end{array}$

To avoid external mass transfer limitations due to the immiscibility between reactants, oil and glycerol, a solvent can be added to the system to create a homogenous phase. In the literature, other studies about solvent-free glycerolysis have been also found (Fregolente et al. 2010; Torres et al. 2002; Weber \& Mukherjee 2004), but the products conversion was quite low due to the limited contact between the reactants.

According to Damstrup et al. (Damstrup et al. 2005), tert-butanol and tert-pentanol resulted to be excellent solvents to carry out these reactions.Their log $P$ values $(0.35$ and 0.89 , respectively) indicate both hydrophilic and hydrophobic characteristics. Moreover, these alcohols do not involve reactions with fatty acids, most likely due to their tertiary structure, which exerts strong steric hindrance to the enzyme. The liquid liquid equilibrium (LLE) data of these systems (sardine oil + glycerol + tert-alcohols) have been determined in a previous work (Solaesa et al. 2013). LLE data help to optimize the amount of solvent needed to create a homogeneous reaction system, since by increasing the amount of solvent in the reaction medium, concentration of substrates at the interface also decreases leading to lower reaction rates.

The most widely used lipases to carry out enzymatic glycerolysis reactions are from Candida antarctica (Novozym 435), Pseudomonas fluorescens (IM-AK), Thermomyces lanuginosus (Lipozyme TL IM) and Rhizomucor miehei (Lipozyme RM IM) (Kristensen et al. 2005). The carrier supports of Lipozyme TL IM and RM IM are hydrophilic. In the presence of a hydrophilic support, the polar reactant, glycerol, is attracted to the hydrophilic 
support and hence the reaction could be inhibited. On the contrary, the carrier support of Novozym 435 is hydrophobic, therefore glycerol does not inhibit the reaction (Yeoh et al. 2009).

Several authors use Novozym 435 as biocatalyst to carry out the glycerolysis reaction due to its highest activity (Pawongrat et al. 2008; Yang et al. 2005a; Yeoh et al. 2009). However, this enzyme is anexpensive lipase compared with other commercial lipases. On the other hand, natural omega-3 PUFA and specifically DHA and DPA are mainly found in fish oil at the sn-2 position of the glycerol backbone (Solaesa et al. 2014). These fatty acids are better adsorbed as monoacylglycerols esterified at the secondary position. Therefore, the use of 1,3 specific lipase would be more convenient than the use of a nonspecific one, as Novozym 435. However, even using sn-1,3 specific lipases, undesirable and unavoidable side reactions of acyl migration could occur to some extent. Acyl migration depends on several parameters, namely reaction temperature, lipase loading, immobilization supports, water content and water activity, solvent type and reaction system (Xu 2003; Xu 2000).

Some commercially available 1,3 specific lipases are from Rhizopus oryzae, Thermomyces lanuginosus or Rhizomucor miehei. Rendón et al (Rendón et al. 2001) studied the glycerolsis of triolein with Lipozyme RM IM and tert-pentanol as reaction medium. They found that water activity was an important factor in the lipase activity. Some water is needed in the reaction medium to keep the catalytic active conformation of the enzyme (Wongsakul et al. 2003). However, an excess of water causes acyl migration leading to a decrease in MAG yield (Singh \& Mukhopadhyay 2012).

In this work enzymatic glycerolysis of sardine oil in two different tert-alcohols (tertpentanol and tert-butanol) as reaction media has been studied. The commercial 1,3specific lipase Lipozyme RM IM (from Rhizomucor miehei) immobilized on a macroporous anion-exchange resin has been used as biocatalyst. In addition to its specificity, Lipozyme RM IM is cheaper than the non-specific Novozym 435 commonly used in enzymatic glycerolysis (Zhong et al. 2009). The effect of water content in the reaction medium has been analyzed since it affects the lipase activity and therefore the reaction rate and MAG 
yield. This way, optimum water content has been determined by taking into account the MAG yield and the FFA formation.

\section{Experimental section}

\subsection{Materials}

The sn-1,3 specific lipase Lipozyme RM IM (immobilized Rhizomucor miehei lipase) was obtained from Sigma Aldrich. The water content of Lipozyme RM IM was $3.5 \pm 0.3 \%$ determined by Karl-Fisher titration with a Mitsubishi CA-20 moisture meter by triplicate. Glycerol was also purchased from Sigma Aldrich with a purity of $\geq 99.5 \%$ and a water content of $0.04 \pm 0.01 \%$. Tert-butanol and tert-pentanol were purchased from Merck with a purity of $\geq 99 \%$ and a water content of $0.298 \pm 0.033 \%$ and $0.065 \pm 0.023 \%$, respectively. Refined sardine oil was kindly provided by Industrias Afines S.L. (Spain). The FA composition of sardine oil and the mole percentage of fatty acid at the sn-2 position of TAG are presented in Table 2.3 (Solaesa et al. 2014). More than $80 \%$ of DHA was found at sn-2 position.

The standards of MAG, DAG, FFA and fatty acid methyl ester (FAME) for chromatography analysis were purchased from Sigma. All other solvents and reagents were of analytical or chromatographic grades from VWR.

\subsection{Enzymatic Glycerolysis of Sardine Oil}

The mixture of sardine oil, glycerol, water and tert-alcohol (TB or TP) was incubated at $50^{\circ} \mathrm{C}$ in a water bath with orbital agitation in different vials. The reaction was initiated by the addition of the lipase. The enzyme loading was $10 \mathrm{wt} \%$, based on total amount of substrates. At different time intervals, the corresponding vial was withdrawn and filtered through a microfilter $(0.45 \mu \mathrm{m}$, Sartorius $\mathrm{RC})$ to stop the reaction by removing the lipase. All samples were stored at $-18^{\circ} \mathrm{C}$ prior to analysis. Samples were analyzed at least by duplicate. 
The conditions of the kinetic experiments performed in this work are presented in Table

2.1. The amount of water added to the system was varied between 4 to $30 \mathrm{wt} \%$, based on glycerol weight, in the reaction medium. The amount of tert-alcohol added has been calculated on the basis on previous studies on liquid liquid equilibrium for glycerolysis systems of sardine oil with the two tert-alcohols as reaction medium (Solaesa et al. 2013).

Table 2.2 shows the amount of solvent added in other glycerolysis systems found in the literature to avoid mass transfer limitation due to the immiscibility of oil and glycerol. Compared to the amount of solvent added in this work (Table 2.1), it can be observed that in most cases the amount of solvent added is not optimized due to an excess or deficiency from the beginning of the reaction.

Table 2.1. Glycerolysis reaction conditions (mole ratio of substrates, water content and amount of tert-alcohol added to the system) at $50{ }^{\circ} \mathrm{C}$ (Solaesa et al. 2013).

\begin{tabular}{ccccccc}
$\begin{array}{c}\text { Reactants mole ratio } \\
\text { (glycerol:oil) }\end{array}$ & $\begin{array}{c}\text { Tert-alcohol } \\
\text { (wt \%) }\end{array}$ & \multicolumn{5}{c}{$\begin{array}{c}\text { Amount of water (wt \% of } \\
\text { glycerol) }\end{array}$} \\
$1: 1$ & 50 & 4 & 7 & 10 & 20 & 30 \\
$3: 1$ & 60 & - & 7 & 10 & 12 & 24 \\
$5: 1$ & 65 & - & 10 & 12 & 17 & 28 \\
\hline
\end{tabular}

\subsection{Determination of reaction products}

The neutral lipid profile (MAG, DAG, TAG and FFA) was analyzed by a normal-phase high performance liquid chromatography (NP-HPLC). The chromatographic apparatus consisted of a HPLC system (Agilent 1200) formed by a quaternary pump and an auto-injector. The chromatographic separation of the compounds was carried out at room temperature with a Lichrospher Diol column $(5 \mu \mathrm{m}, 4 \mathrm{~mm} \times 250 \mathrm{~mm}$ ) and detection was performed in an evaporative light scattering detector (Agilent 1200 series) at $35^{\circ} \mathrm{C}$ and $0.35 \mathrm{MPa}$. Gradient elution was achieved by mobile phases A (isooctane) and B (methyl tert-butyl ether:acetic acid $=99.9: 0.1, v / v)$. The course of the gradient was as follows: first, solvent $A$ was flowing for $1 \mathrm{~min}$, after that, solvent $B$ was added in three steps, up to $10 \%$ in $10 \mathrm{~min}$, to $44 \%$ in $22 \mathrm{~min}$ and to $100 \%$ in $30 \mathrm{~min}$. Subsequently, solvent B was decreasing down to $0 \%$ in 17 
min to come back to the initial conditions. Finally, the stationary phase was rinsed with solvent A for $2 \mathrm{~min}$. Injection volumes of $10 \mu \mathrm{L}$ and the elution flow-rate of $1 \mathrm{~mL} / \mathrm{min}$ were used in all experiments. The different family of compounds were identified and quantified by using calibration curves of the corresponding standard compounds of DAG, a mixture of dipalmitinand diolein, MAG, a mixture of 1-monopalmitin, 1-monoolein, 2-monooleinand monodocosahexaenoin and FFA, a mixture of oleicacid and palmitic acid in tert-pentanol. In case of TAG, the calibration curve used was made with the refined sardine oil (TAG $\geq 99 \%$ ) because the response factor of the oil as a complex mixture of TAG was very different than the pure standards of TAG. The calibration method has been validated by analyzing two different samples formed by MAG, DAG and FFA standards and sardine oil in the concentration range of the corresponding calibration curves obtaining good reliability.

Table 2.2. Amount of tert-alcohol added in others glycerolysis systems found in the literature.

\begin{tabular}{|c|c|c|c|c|}
\hline Oil & $\begin{array}{l}\text { Mole ratio } \\
\text { (glycerol:oil) }\end{array}$ & $\mathrm{T}\left({ }^{\circ} \mathrm{C}\right)$ & $\begin{array}{c}\% \text { wt of tert- } \\
\text { alcohol }\end{array}$ & Reference \\
\hline Palm & $8: 1$ & 45 & $82 \%$ of TB & $\begin{array}{c}\text { (Majid \& } \\
\text { Cheirsilp 2012) }\end{array}$ \\
\hline Olive & $\begin{array}{c}6: 1,3: 1,0.8: 1 \\
0.5: 1.5\end{array}$ & $40,55,70$ & $44 \%$ of TB & $\begin{array}{c}\text { (Voll et al. } \\
\text { 2011) }\end{array}$ \\
\hline Olive & $9: 1,6: 1,3: 1,1: 1$ & $40,55,70$ & $44 \%$ and $80 \%$ of TB & $\begin{array}{c}\text { (Krüger et al. } \\
\text { 2010) }\end{array}$ \\
\hline Triolein & $2: 1$ & 40 & $93 \%$ of TP & $\begin{array}{c}\text { (Rendón et al. } \\
\text { 2001) }\end{array}$ \\
\hline
\end{tabular}

The analysis of remaining glycerol was performed by using High-Temperature Gas Chromatography (HT-GC). The method and the calibration procedure were previously developed (Solaesa et al. 2013). 


\subsection{Fatty acid profile of MAG fractions}

The MAG fraction separated by NP-HPLC under the optimal reaction conditions with both solvents was collected according to its retention time. Separations were repeated at least six times to obtain enough sample for the fatty acid profile analysis. The fractions were stored in special flasks to evaporate the solvent under vacuum using a rotary evaporator (Heibolph VV2000) at $40^{\circ} \mathrm{C}$. Then, the samples were transferred to screw-capped tubes to carry out the derivatization for conversion to methyl esters by the AOAC method (AOAC Official Method 991.39 2000)and subsequent analysis by Gas Chromatography with an Agilent gas chromatograph (6890N Network GC System) equipped with a flame ionization detector (FID). A fused silica capillary column (OmegawaxTM-320, $30 \mathrm{~m} \times 0.32 \mathrm{~mm}$ i.d.) was used. The separation was carried out with helium $(1.8 \mathrm{~mL} / \mathrm{min})$ as carrier gas. The column temperature was programmed starting at a constant value of $180{ }^{\circ} \mathrm{C}$ during $20 \mathrm{~min}$, heated to $200{ }^{\circ} \mathrm{C}$ at $1{ }^{\circ} \mathrm{C} / \mathrm{min}$, held at $200^{\circ} \mathrm{C}$ during $1 \mathrm{~min}$, heated again to $220^{\circ} \mathrm{C}$ at $5{ }^{\circ} \mathrm{C} / \mathrm{min}$ and finally held at $220^{\circ} \mathrm{C}$ for $20 \mathrm{~min}$. A split injector (50:1) at $250{ }^{\circ} \mathrm{C}$ was used. The FID was also heated at $250^{\circ} \mathrm{C}$. The injection volume was $1 \mu \mathrm{L}$. Most of the fatty acid methyl esters were identified by comparison of their retention times with those of chromatographic standards (Sigma Chemical Co.). The calibration procedure was previously developed and quantification was possible relating the peaks area to the area of an internal standard (methyl tricosanoate) as indicated by the AOAC method (AOAC Official Method 991.39, 1995).

\subsection{Isomeric separation of reaction products}

The separation of the different acylglycerols has been also performed by TLC on silica gel plates (Silica gel 60 F254, Merck). A similar method of Jin et al. (Jin et al. 2011) was used to visualize sn-1(3) and sn-2-MAG isomers. The plates were impregnated by immersion in a hydroethanolic solution (50 \%, v/v) of boric acid (1.2 wt \%). Afterwards, they were activated by heating at $100{ }^{\circ} \mathrm{C}$ for $15 \mathrm{~min} .20 \mu \mathrm{L}$ of the reaction mixture were directly spotted on the TLC plate. The plates were then developed in chloroform/acetone/methanol (95:4.5:0.5, v/v/v). Spots of each lipid were visualized by UV lamp. 


\section{Results and discussion}

In a first step, glycerolysis of sardine oil using both tert-alcohols as reaction media, but without any enzyme as catalyst has been performed. The experimental conditions were the following: $50{ }^{\circ} \mathrm{C}$, initial mole ratio glycerol to oil 3:1 and solvent to substrates ratio of 1.2:1 w/w. It was observed that glycerolysis reaction does not present an auto-catalytic behavior, since under these experimental conditions the observed reaction rate was almost zero. Yang et al. (Yang et al. 2005b) showed that, at room temperature, without the presence of any catalyst, the reaction rate for this reactive system is generally very slow, and the substrates may need weeks to be transformed into the products. The same behavior was also verified by Krüger et al. (Krüger et al. 2010) at $70^{\circ} \mathrm{C}$.

In this work, further glycerolysis experiments were performed at $50{ }^{\circ} \mathrm{C}$, solvent to substrates ratio of 1.2:1 wt/wt and $10 \mathrm{wt} \%$ of Lipozyme RM IM loading at different glycerol to oil mole ratios (1:1, 3:1 and 5:1). In these experiments no water was added and it was observed that the glycerolysis reaction did not occur. This might be because the carrier of Lipozyme RM IM is hydrophilic and the glycerol, as hydrophilic substrate, can be preferably adsorbed on the support depraving the essential water from the enzyme. Therefore, water content in the reaction medium must be an essential factor in the activity of Lipozyme RM IM. In the next section the effect of water addition in the glycerolysis system catalyzed by Lipozyme RM IM will be presented and discussed.

\subsection{Effect of water}

As it has been already mentioned, the carrier support of Lipozyme RM IM is hydrophilic. In the presence of a hydrophilic support, the tert-alcohol and mainly glycerol $(\mu=2.617$, $\log P=-1.84$ and $\left.\varepsilon\left(23^{\circ} \mathrm{C}\right)=41.01\right)$ (Riddick et al. 1986) can be preferably adsorbed on the support. Due to the hydrophilic nature of the reaction medium, some essential water from the lipase can be removed. Therefore, it is important to determine the optimal water content in the reaction medium since it affects lipase activity and therefore the reaction rate and yield product. On the other hand, when the water content is too high does not enhance the MAG yield resulting in an increase of by-product formation, such as FFA 
(Zhong et al. 2009). Therefore water control is important to ensure enzyme activity as well as to minimize hydrolytic side reactions.

First, some preliminary experiments were carried out to study the effect of the polarity medium in the retention of titrable water by the enzyme. The moisture of different reaction media (tert-alcohol + glycerol + sardine oil) at different reactant mole ratio glycerol:oil, from 1:1 to 6:1 was first determined. Then, Lipozyme RM IM with a known amount of titrable water, was added at $10 \% \mathrm{w} / \mathrm{w}$ based on reactants weight. After 24 hours of contact time at $50{ }^{\circ} \mathrm{C}$ (time enough to reach sorption equilibrium) the water content of the reaction medium was again determined. Total titrable water retention by Lipozyme RM IM was evaluated according to Equation 4:

$\%$ water retention $=\left(1-\frac{\text { increase of water in the reaction medium }}{\text { amount of titrable water of the lipase }}\right) \cdot 100$ [4]

Figure 2.1 shows the titrable water retention by Lipozyme RM IM in both tert-alcohols studied in this work. By increasing the amount of glycerol in the reaction medium (higher glycerol:oil mole ratio) more water is removed from the lipase surrounding. This can be related to the higher polarity of the reaction medium when increasing the glycerol content. This behavior could explain previous results on glycerolysis kinetics where no reaction took place even in the presence of Lipozyme RM IM as catalyst, proving that this lipase is water-dependent. When comparing both tert-alcohols, tert-butanol is more hydrophilic than tert-pentanol $(\log P=0.35$ and $\log P=0.89$, respectively), therefore tertbutanol medium deprives more water from the enzyme than TP medium under the same conditions. Consequently, TB as reaction medium would need more added water than TP to obtain similar results under the same conditions.

The effect of water content in the reaction medium on MAG and FFA yield at three different initial reactant mole ratios (glycerol:oil), 1:1, 3:1 and 5:1, was studied at $50{ }^{\circ} \mathrm{C}$ by varying the amount of water added to the reactive system in the range of $4-30 \mathrm{wt} \%$ of water based on glycerol weight (Table 2.1). 


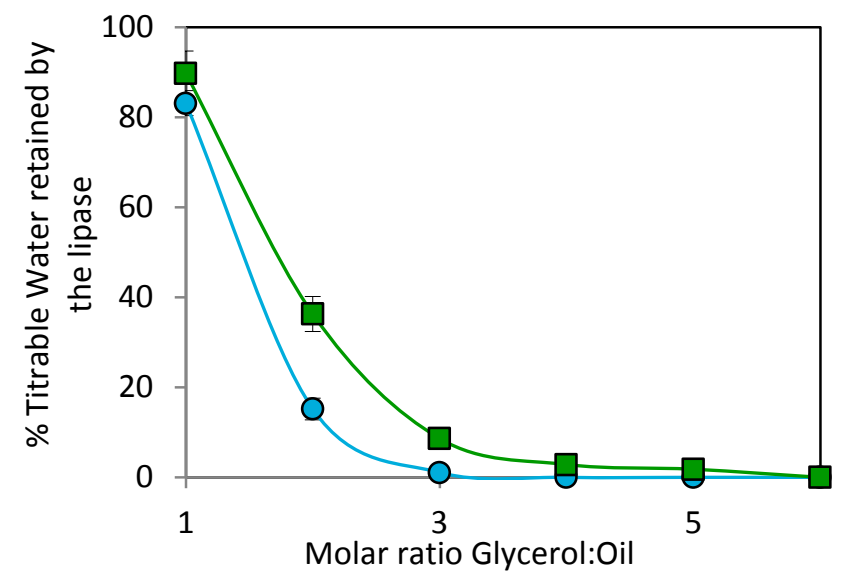

Figure 2.1.Titrable water retention by Lipozyme RM IM at different initial mole ratio glycerol:oil in both tert-alcohols, (•) tert-butanol and ( $\square$ ) tert-pentanol. Lines are to guide the eye.

Figure 2.2 shows the MAG and FFA content obtained by using tert-butanol and tertpentanol as reaction medium after $20 \mathrm{~h}$ reaction time. As a general trend, MAG yield increased with the increase of water content in the reaction medium up to a maximum. The highest yield of MAG was obtained by adding around 7-17 wt\% of water, depending on the substrate mole ratio. For instance at a mole ratio of $1: 1$ the percentage of water added to yield a maximum in the MAG production was around $7 \mathrm{wt} \%$ in TP as reaction medium and $10 \mathrm{wt} \%$ in TB as reaction medium. This behavior agrees with the results presented in Figure 2.1. However at a mole ratio of 5:1 the optimum percentage of water added for both tert-alcohols was of the same order, around $17 \mathrm{wt} \%$. This could be explained by taking into account that at such high mole ratio most of the essential water of the enzyme has been deprived (Figure 2.1) and the effect of solvent is less pronounced. An increase of the amount of water in the reaction medium also leads to an increase in the FFA content, caused by higher hydrolysis rates due to the higher content of water. At a mole ratio of 3:1 FFA content in the reaction medium increased from 5.3 to $32.5 \mathrm{wt} \%$ when increasing the amount of added water from 7 to 24 wt\%, respectively. Therefore it exits an optimal amount of water added to the reaction medium taking into account the MAG yield and the FFA formation at the different mole ratios glycerol:oil. In addition, mass transfer limitations of the lipophilic substrate, the sardine oil, from the reaction 
medium to the vicinity of the enzyme can become important at high water content since, as described in the literature (Cheirsilp 2007; Zhong et al. 2009), an excess of water can surround the particles instead of forming a thin mono hydration layer for catalyticallyactive conformation. Zhong et al. (Zhong et al. 2009) found that the highest yield of MAG was obtained when $10 \mathrm{wt} \%$ of water was added to the glycerolysis sytem of soybean oil with Thermomyces lanuginosus lipase and TB/Isopropanol (80:20) as solvent system.

\subsection{Effect of substrate mole ratio}

Substrate mole ratio, glycerol:oil, has different effects on lipase-catalyzed glycerolysis. According to the equilibrium law, an increase in the glycerol content will shift the equilibrium towards MAG production. In the literature, it has been also described that glycerol can act as an effective stabilizer against thermal and solvent deactivation (Zhong et al. 2009). However, in the case of water-dependent lipases, the glycerol content can also directly affects the lipase activity due to its influence on the polarity of the reaction medium.

The effect of glycerol:oil mole ratio on the reaction rate was studied at $50{ }^{\circ} \mathrm{C}, 10$ wt\% of lipase loading, by varying the mole ratio from 1:1 to 5:1. Figure $\mathbf{2 . 3}$ shows the MAG profile at the different glycerol:oil mole ratio and at high content of water added (from 24 to 30 $w t \%$ in base of glycerol content), for both tert-alcohols. By increasing the initial substrate mole ratio, MAG equilibrium conversion also increases. For instance MAG content at a mole ratio of $1: 1$ is around $50 \%$ in tert-butanol, while it reaches a maximum of $75 \%$ at a mole ratio of 5:1. However, it must be highlighted that there is little difference in MAG contentat 3:1 and 5:1 glycerol:oil mole ratio. Based on these results, glycerol:oil mole ratio of 3:1 was selected as optimum in the present study. This result agrees with some previous studies on glycerolysis of different types of oils. Zhong et al. (Zhong et al. 2009) selected 3.5:1, as optimum glycerol:soybeanoil mole ratio, in their study on glycerolysis reaction in a mixture of TB/Isopropanol (80:20) as reaction medium. Pawongrat et al. (Pawongrat et al. 2007) used MTBE as solvent and showed that the optimum mole ratio of glycerol to tuna oil for MAG production was 3:1. 

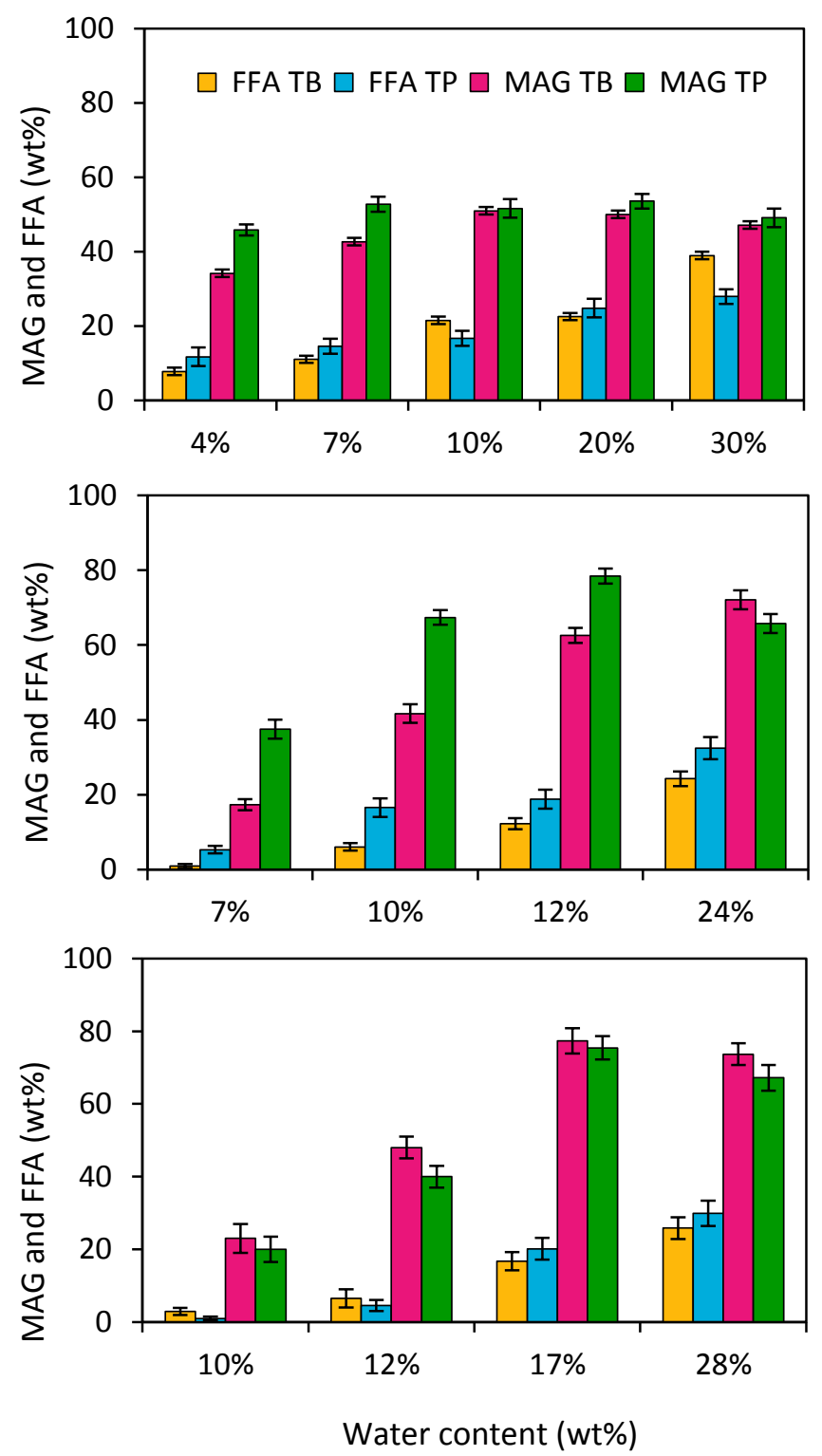

Figure 2.2. Effect of water content, based on glycerol weight, on glycerolysis of sardine oil at different initial mole ratio glycerol:oil (a) 1:1 (b) 3:1 and (c) 5:1. Reaction conditions:

10 wt\% Lipozyme RM IM (based on substrates), $50{ }^{\circ} \mathrm{C}$ at $20 \mathrm{~h}$. 

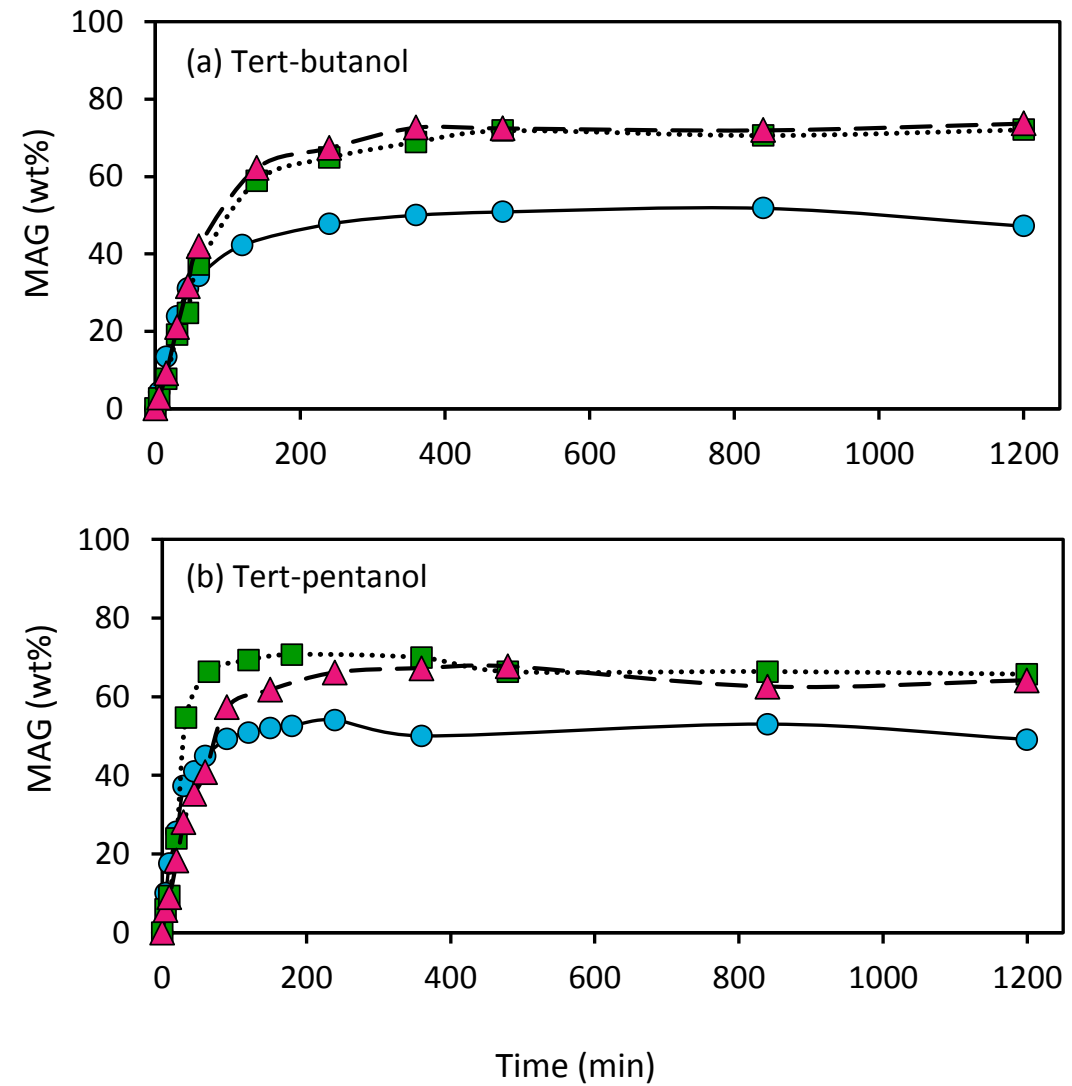

Figure 2.3. Effect of substrate mole ratio on the time course of glycerolysis of sardine oil

(a) TB, (b) TP at different initial mole ratio glycerol:oil (-๑-) 1:1, (-口-) 3:1, (- $\Delta$-) 5:1.

Reaction conditions: 10 wt\% Lipozyme RM IM (based on oil and glycerol), $50{ }^{\circ} \mathrm{C}$ and $24-30$ wt\% of added water based on glycerol weight (see Figure 2.2).

\subsection{Effect of solvent type}

Solvents can have different effects on reaction systems. They help to create homogeneous reaction system and improve the mass transfer by reducing the viscosity of reaction medium. When polar solvents are used, essential water from the lipase can be removedleading to a decrease in the lipase activity. In this work two tertiary alcohols, tertbutanol and tert-pentanol, have been considered as reaction media. Log $\mathrm{P}$ is one of the most widely used parameters to correlate the solvent properties to the enzyme activity. Taking into account the log P of TB and TP ( 0.35 and 0.89 respectively), TB is slightly more 
hydrophilic than TP; therefore water retention by the lipase will be lower when using TB as reaction medium and slower reaction rates can be expected.

Figure 2.4 shows the effect of both tert-alcohols on the time course of glycerolysis of sardine oil under the same reaction conditions at different amounts of water added to the reaction medium. As expected, reaction rates in TB were slower than in TP. Nevertheless, MAG equilibrium yield was of the same order for both tert-alcohols.

\subsection{MAG production under optimal conditions}

Based on the results presented in this work, the optimal conditions for MAG production with 10 wt\% loading of Lipozyme RM IM, based on substrate weight, at $50{ }^{\circ} \mathrm{C}$ were the following: initial mole ratio glycerol:oil 3:1, water content in glycerol $12 \mathrm{wt} \%$ and $60 \mathrm{wt} \%$ of the corresponding tert-alcohol to provide a homogeneous medium. Mole ratio higher than 3:1 does not lead to an increase in MAG yield (Figure 3). At this mole ratio, a maximum in MAG yield (around $78 \mathrm{wt} \%$ ) in TP is reached at $12 \mathrm{wt} \%$ of water added, based on glycerol weight, with less than 20 wt\% of FFA (Figure 2.2). The time course of glycerolysis of sardine oil for all the compounds in both tert-alcohols under the optimal conditions is presented in Figure 2.5.

Figure 2.5a and 2.5b represent the glycerolysis course in TB and TP respectively. From Figure 2.5a can be observed that MAG contentin TB as reaction medium was higher than 65 wt\% after 20 hours of reaction time. At this reaction time TAG content in the reaction medium was still about 20 wt\%. DAG production slightly increased during the first 4 hours and then it remained relatively constant around $7 \mathrm{wt} \%$. At the beginning of the reaction, FFA formation is nearly negligible and then it rose slowly up to 10 wt\%.

From Figure 2.5b can be observed that reaction rate was faster in TP than in TB. TAG decreased sharply during the first $2 \mathrm{~h}$ and then, the reaction rate became slower. MAG content also increased quickly during the first $2 \mathrm{~h}$ reaching a value of $60 \mathrm{wt} \%$. From this time on, reaction rate slowed down and MAG content reached a maximum value of $78 \mathrm{wt} \%$. DAG production achieved a maximum at 2-3 $\mathrm{h}$ reaction time and then its content steadily decreased. FFA formation is also faster in TP than in TB. 


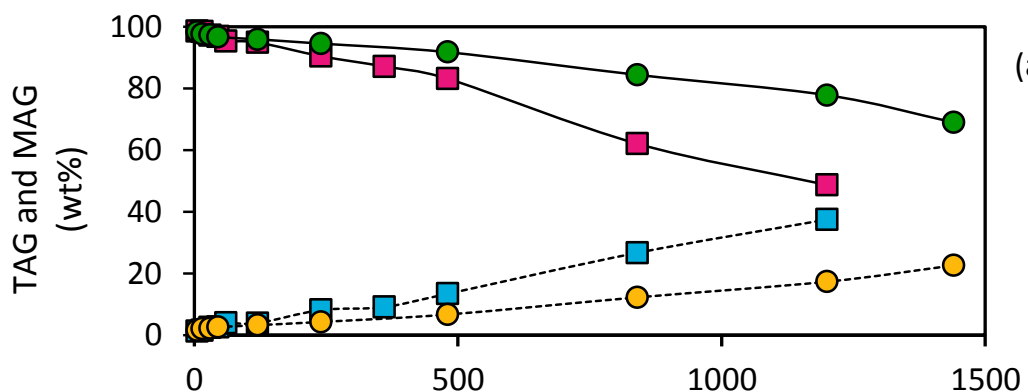

(a) $7 \%$ water

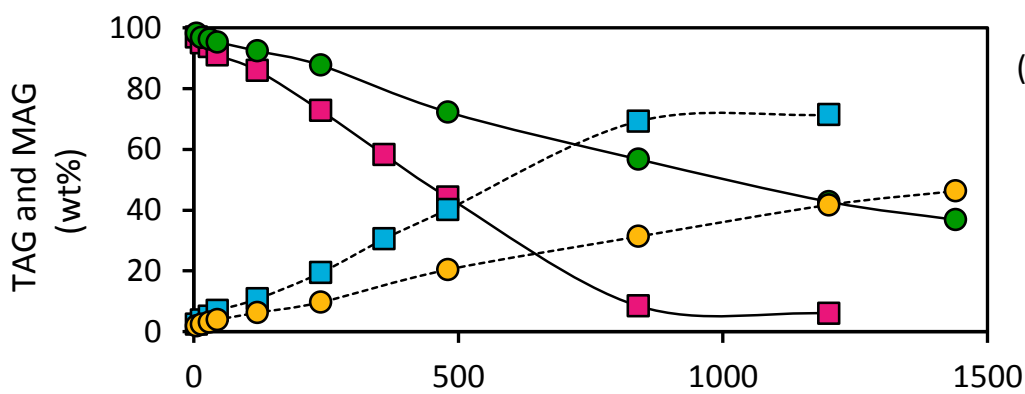

(b) $10 \%$ water

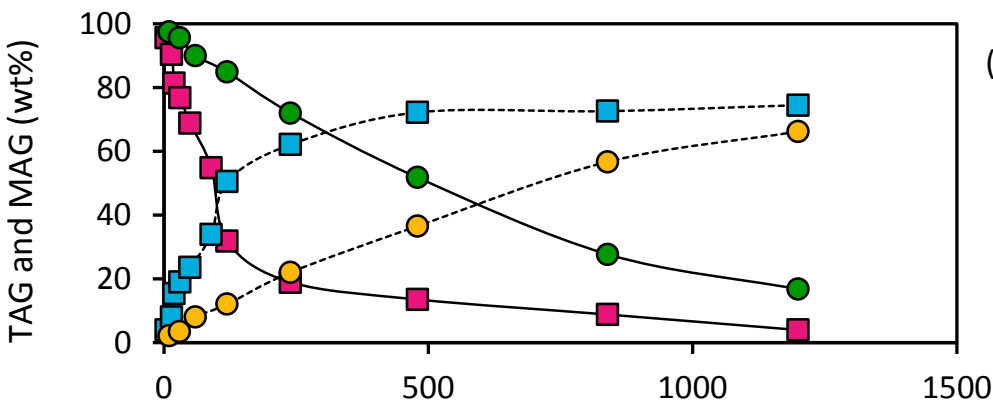

(c) $12 \%$ water

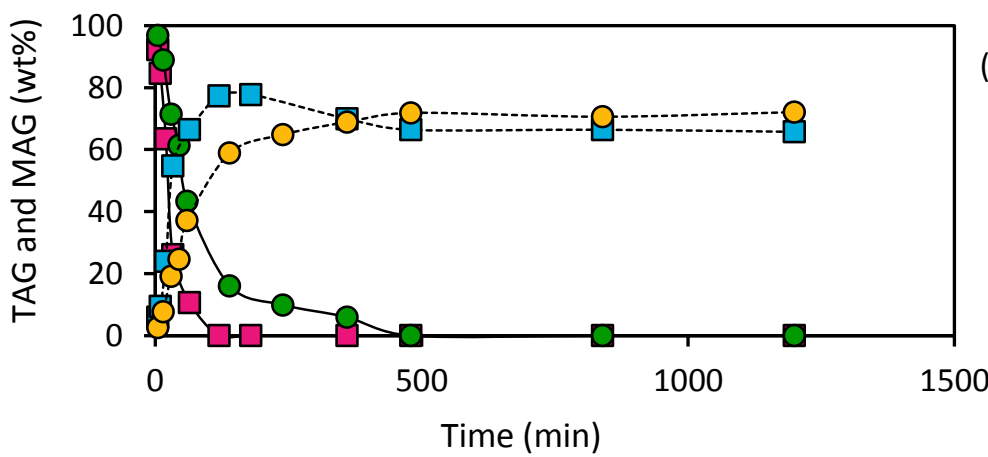

(d) $24 \%$ water

Figure 2.4. Effect of solvent type and water content on the time course of glycerolysis of sardine oil at different amounts of water in TP (grey lines) and in TB (black lines). (a)

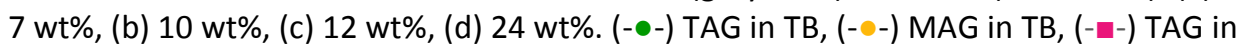
TP, (-m-) MAG in TP. Reaction conditions: glycerol:oil = 3:1 (mol $/ \mathrm{mol}), 10 \mathrm{wt} \%$ Lipozyme RM IM (based on oil and glycerol), $50^{\circ} \mathrm{C}$. 

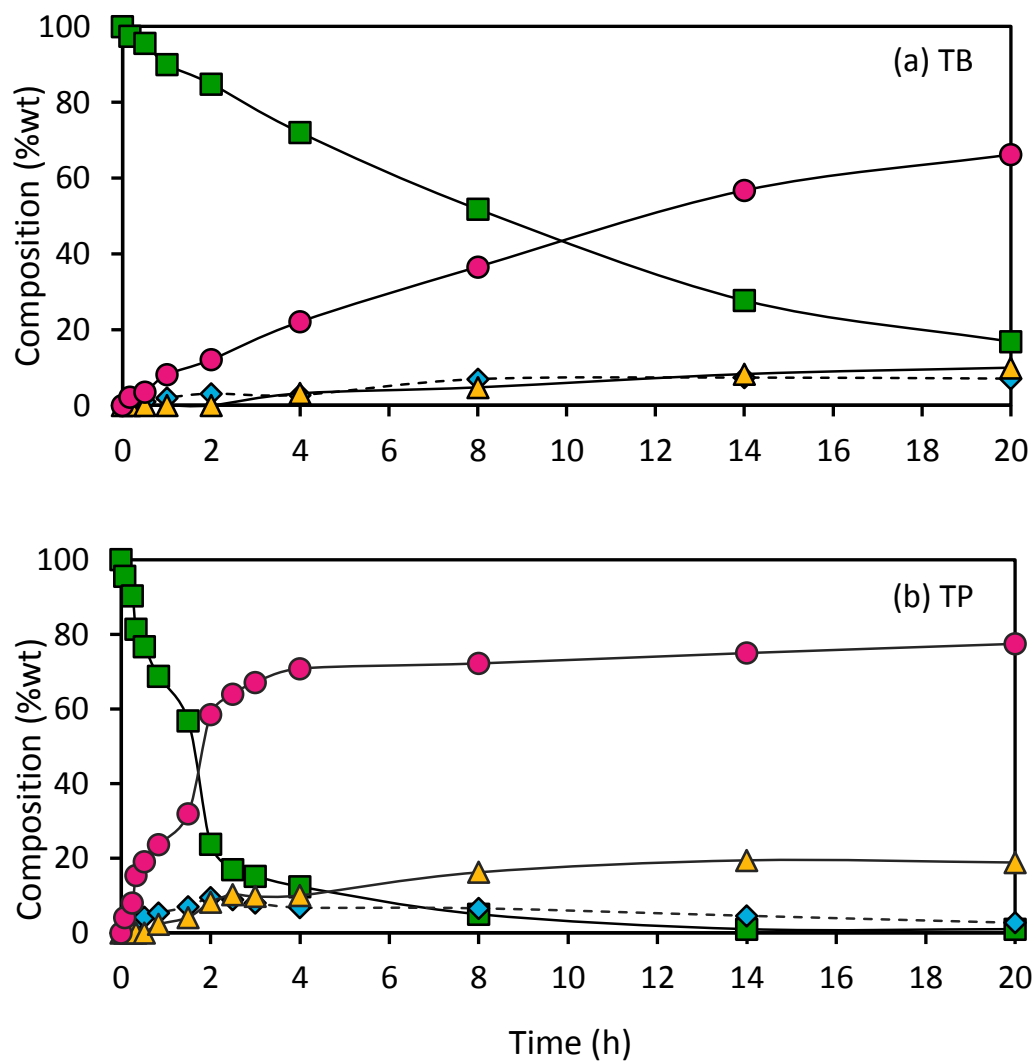

Figure 2.5. Time course of glycerolysis of sardine oil under optimal conditions: glycerol/oil $=3: 1(\mathrm{~mol} / \mathrm{mol}), 10 \mathrm{wt} \%$ Lipozyme RM IM (based on oil and glycerol), $50{ }^{\circ} \mathrm{C}$ and $12 \%$ of water content in the glycerol. (a) in TB and (b) in TP. TAG (---), MAG (-๑-), DAG (- -) and FFA (- $\triangle-)$.

FA profile of different MAG fractions obtained under the optimal conditions was analyzed by GC. MAG fractions at two different reaction times ( $4 \mathrm{~h}$ and $20 \mathrm{~h}$ ) were separated by HPLC and collected to determine their fatty acid profiles (Table 2.3). The MAG content in TP as reaction medium at the selected reaction times, $4 \mathrm{~h}$ and $20 \mathrm{~h}$, was $70 \mathrm{wt} \%$ and $77 \mathrm{wt} \%$ respectively. On the other hand, in TB as reaction medium, the MAG content was 22 and 66 wt\% at 4 and 20 hours, respectively. Much longer reaction time is needed in TB than in TP to reach the same MAG percentage in the reaction medium. 
Tabla 2.6. Fatty acid composition of sardine oil and at the sn-2 position of TAG (\% mol) (Solaesa et al. 2014). Fatty acid composition of MAG fractions after NP-HPLC separation under optimal reaction conditions ( $\% \mathrm{~mol}$ ).

\begin{tabular}{|c|c|c|c|c|c|c|c|}
\hline \multirow{2}{*}{ Fatty acid } & & \multicolumn{2}{|c|}{ Sardine oil } & \multicolumn{2}{|c|}{ TP medium } & \multicolumn{2}{|c|}{ TB medium } \\
\hline & & TAG & $s n-2^{a}$ & $4 h(70 \%)$ & $20 \mathrm{~h}(77 \%)$ & $4 h(22 \%)$ & $20 \mathrm{~h}(66 \%)$ \\
\hline Myristic (M) & $14: 0$ & 12.4 & 41.8 & $12.2 \pm 0.3$ & $12.0 \pm 0.2$ & $12.3 \pm 0.5$ & $11.9 \pm 0.5$ \\
\hline Palmitic (P) & $16: 0$ & 22.8 & 41.9 & $25.2 \pm 0.3$ & $25.1 \pm 0.1$ & $27.1 \pm 0.6$ & $25.6 \pm 0.2$ \\
\hline Palmitoleic (Po) & $16: 1 n-7$ & 12.5 & 38.1 & $13.4 \pm 0.3$ & $12.6 \pm 0.2$ & $13.5 \pm 0.1$ & $13.3 \pm 0.2$ \\
\hline Stearic (S) & 18:0 & 3.6 & 6.7 & $4.4 \pm 0.4$ & $4.3 \pm 0.1$ & $6.0 \pm 0.2$ & $4.6 \pm 0.1$ \\
\hline Oleic (O) & $18: 1 n-9$ & 9.8 & 16.6 & $12.3 \pm 0.3$ & $11.5 \pm 0.3$ & $15.3 \pm 0.3$ & $12.4 \pm 0.5$ \\
\hline Vaccenic (V) & $18: 1 n-7$ & 3.7 & 10.1 & $4.3 \pm 0.2$ & $4.1 \pm 0.1$ & $5.0 \pm 0.1$ & $4.3 \pm 0.1$ \\
\hline Linoleic (Lo) & $18: 2 n-6$ & 2.5 & 32.7 & $3.0 \pm 0.2$ & $2.7 \pm 0.1$ & $3.6 \pm 0.7$ & $3.0 \pm 0.1$ \\
\hline Linolenic (Ln) & $18: 3 n-3$ & 1.0 & 29.0 & $1.1 \pm 0.1$ & $1.0 \pm 0.1$ & $1.2 \pm 0.1$ & $1.1 \pm 0.1$ \\
\hline Steriadonic (St) & $18: 4 n-3$ & 3.3 & 26.4 & $1.9 \pm 0.2$ & $2.5 \pm 0.2$ & $1.1 \pm 0.1$ & $1.8 \pm 0.1$ \\
\hline Eicosatrienoic (Et) & $20: 3 n-3$ & 1.3 & 19.6 & $1.0 \pm 0.1$ & $1.2 \pm 0.1$ & $0.7 \pm 0.1$ & $1.0 \pm 0.1$ \\
\hline Eicosapentaenoic (Ep) & $20: 5 n-3$ & 18.3 & 12.1 & $14.4 \pm 0.3$ & $15.5 \pm 0.2$ & $10.1 \pm 0.2$ & $14.3 \pm 0.2$ \\
\hline Docosapentaenoic (Dp) & $22: 5 n-3$ & 1.8 & 76.4 & $1.7 \pm 0.1$ & $1.8 \pm 0.1$ & $1.1 \pm 0.1$ & $1.7 \pm 0.1$ \\
\hline Docosahexaenoic (Dh) & $22: 6 n-3$ & 7.0 & 82.8 & $5.1 \pm 0.2$ & $5.7 \pm 0.2$ & $3.0 \pm 0.1$ & $5.0 \pm 0.2$ \\
\hline
\end{tabular}

(a) $\% s n-2=[\mathrm{mol} \% s n-2$ fatty acid / (mol \% fatty acid in TAG·3) $] \cdot 100$ 
From Table $\mathbf{2 . 3}$ can be observed a similar fatty acid profile of the MAG fraction obtained in tert-butanol at $20 \mathrm{~h}$ of reaction time (66 wt\% of MAG) as in tert-pentanol at $4 \mathrm{~h}$ of reaction time (70 wt\% of MAG). Although reaction rate is slower in $T B$, it could be concluded that Lipozyme RM IM would behave qualitatively in the same way according to MAG conversion in both tert-alcohols. Figure $\mathbf{2 . 6}$ represents the different types of FA in the MAG fractions as a function of the MAG content in the reaction medium for both tertalcohols. PUFA content in the MAG fraction increased when the conversion degree also increased; while monounsaturated FA (MUFA) and saturated FA (SFA) decreased.

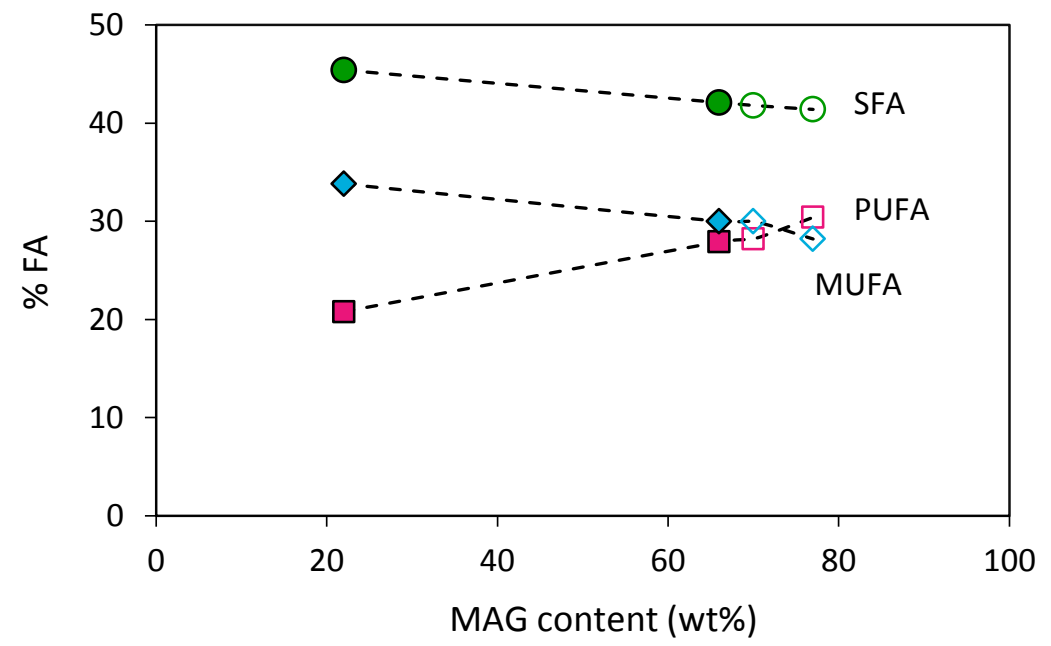

Figure 2.6. Evolution of the different types of FA in MAG fraction according to the conversion degree. Filled symbols represent reaction with TB and empty symbols with TP.

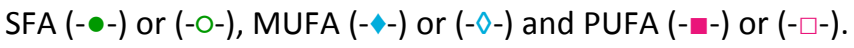

TLC analyses have been performed at certain reaction times (from 0.5 to $20 \mathrm{~h}$ ) to check the formation of 1(3)-MAG and 2-MAG isomers. The FA profile of 1(3)-MAG and 2-MAG fractions at $8 \mathrm{~h}$ of reaction time was known by extraction of the appropriate band and the subsequent methylation for the FAME analysis in GC (data not shown). The results showed that the majority of EPA is found as 1(3)-MAG, while DHA is found mainly in 2-MAG. These outcomes are in agreement with the FA at the sn-2 position in the TAG (Table 2.2). In any case, acyl-migration could take place due to the positive surface charge of the support material, Duolite 568, the water added and the solvent medium (Fureby et al. 1996). 


\section{Conclusions}

In this work, glycerolysis of sardine oil in two different tert-alcohols (TP and TB) as reaction medium has been performed. The amount of solvent added has been optimized to ensure the homogeneity of the reaction medium. The 1,3 specific lipase Lipozyme RM IM has been used as catalyst. This enzyme has been proved to be water dependent in this glycerolysis system, showing no activity without water and a maximum in the MAG production when adding around $12 \%$ of water, based on glycerol, at a mole ratio glycerol:oil of 3:1. High content of MAG, up to $70 \mathrm{wt} \%$, can be reached for both tertalcohols, with a low FFA content. Furthermore, the fatty acid profile of 1(3)-MAG and 2MAG fractions show that the original FA at the sn-2 position in the TAG is preserved, founding the majority of DHA as 2-MAG.

This research could be an economical alternative to take advantage of by-products of marine oils rich in PUFA. Anyway, further work must be done to purify the MAG derivatives rich in PUFA due to their health benefits to be uses in the pharmaceutical and food industries. 



\section{CHAPTER 3}

\section{Kinetic study and kinetic parameters of lipase-catalyzed glycerolysis of sardine oil in a homogeneous medium}

Á. García Solaesa, M.T. Sanz, S. Beltrán and R. Melgosa (2016).

"Kinetic study and kinetic parameters of lipase-catalyzed glycerolysis of sardine oil in a homogeneous medium"

Chinese Journal of Catalysis, 37, 596-606 



\section{Capítulo 3}

\section{Estudio cinético y parámetros cinéticos de la glicerolisis de aceite de sardina catalizada por una lipasa en un medio homogéneo.}

\section{Resumen}

Para obtener concentrados de AGPI en forma de diglicéridos y monoglicéridos (DAG y $M A G)$, en este capítulo se ha estudiado la glicerolisis enzimática de aceite de sardina con la lipasa comercial e inmovilizada Lipozyme 435 de Candida antarctica como biocatalizador y con tert-butanol como medio de reacción. La cantidad de disolvente se ha optimizado según el equilibrio líquido-líquido estudiado para este sistema en el capítulo 1. En primer lugar se analizaron las limitaciones por transferencia de materia del sistema de reacción. También se han estudiado los efectos de diferentes variables de operación como la carga de la lipasa, temperatura de reacción y relación inicial de sustratos y tert-butanol. Se ha utilizado un modelo cinético semi-empírico basado en las reacciones elementales reversibles de la glicerolisis e hidrolisis de los glicéridos para correlacionar los datos cinéticos experimentales. La relación molar glicerol:aceite de sardina determinada como óptima fue de $3: 1$, con la que se obtuvieron $84 \%$ de MAG a $50^{\circ} \mathrm{C}$. También se ha llevado a cabo un estudio comparativo con otros sistemas de glicerolisis relacionando el rendimiento en MAG, la velocidad de la reacción y los parámetros cinéticos más importantes. 

Abstrat

The production of polyunsaturated fatty acids (PUFAs) concentrates by enzymatic catalysis has gained interest in the last years due to their stereospecificity and the milder conditions involved when compared to inorganic catalysis. In this work, enzymatic glycerolysis of sardine oil by Lipozyme 435 in an optimized amount of tert-butanol as organic solvent has been studied to obtain PUFA concentrates in the forms of di- and monoacylglycerols (DAGs, MAGs). First, mass transfer limitations in the reaction system were analyzed. The effect of different operating variables such as lipase loading, temperature and feed composition was investigated. A semi-empirical kinetic model based on the reversible elementary reactions of glycerolysis and hydrolysis of the glycerides was employed to successfully correlate the experimental kinetic data. A mole ratio glycerol:oil of 3:1 has been considered the optimum to produce more than $84 \mathrm{wt} \%$ of MAG at $50^{\circ} \mathrm{C}$. Comparison with other glycerolysis systems has been also performed in terms of MAG yield, reaction rate and significance of kinetic parameters.

1. Introduction

Fish oil is rich in omega-3 (n-3) polyunsaturated fatty acids (PUFAs), such as eicosapentaenoic acid (EPA) and docosahexaenoic acid. Health benefits of $n-3$ fatty acids have been widely established in the literature (De Deckere et al. 1998; Kris-Etherton et al. 2002; Nichols et al. 2014). Among the different types of lipid derivatives containing PUFA concentrates, MAG and DAG present a good bioavailability (Hernandez 2014; Lawson \& Hughes 1988b). Additionally, MAG or their mixtures with DAG account for approximately $75 \%$ of worldwide emulsifier production (Zhong et al. 2009). The process currently used in industry to obtain MAG is glycerolysis by inorganic alkaline catalyst at high temperatures $\left(220-260^{\circ} \mathrm{C}\right)$. This method presents several disadvantages such as dark color and burnt taste development as well as high energy consumption. Furthermore chemical glycerolysis is not suitable to produce MAG rich in PUFA due to oxidization problems. Enzymatic glycerolysis is an attractive alternative for production of MAG rich in PUFA since reaction can be carried out under mild conditions (Bornscheuer 1995) and structured products can be obtained. 
The immiscibility of reactants, glycerol and oil, leads to masstransfer limitations in the glycerolysis of oils. Different approaches have been considered in the literature to improve the contact between reactants and hence reduce mass transfer limitations. This way lipase-catalyzed glycerolysis has been carried out in different reaction media such as organic solvents (Damstrup et al. 2005), compressed fluids (Moquin et al. 2005), or ionic liquids (Guo \& Xu 2006) in order to improve mass transfer. Recently the use of different surfactants that increase the interfacial area (Valério, Krüger, et al. 2009) and ultrasound irradiation (Fiametti et al. 2012) has been also proposed to reduce mass transfer limitations.

This paper is part of a wider project considering the optimization of MAG production by enzymatic glycerolysis of sardine oil. First, the use of different tert-alcohols has been evaluated as solvents to help to create a homogeneous phase (Solaesa et al. 2013). Tertiary alcohols enhance enzyme activity and accelerate the reaction rate compared to solvent-free system (Damstrup, Abildskov, et al. 2006). In a previous work, tert-pentanol was selected as solvent and the effect of glycerol:oil mole ratio was evaluated in the kinetic behavior as well as in terms of MAG yield. The glycerolysis product was subsequently fractionated by 2-step molecular distillation to obtain a concentrated product of MAG and DAG rich in PUFA (Solaesa, Sanz, Falkeborg, et al. 2016). In this work, a different tertiary alcohol, tert-butanol, has been used as solvent. Tert-butanol has been used in different glycerolysis systems of vegetable oils such as olive oil (Krüger et al. 2010; Voll et al. 2011), palm oil (Majid \& Cheirsilp 2012), camellia oil (Zeng et al. 2010)and sunflower oil (Damstrup et al. 2005; Yang et al. 2005a).

The main objective of this work is to present a detailed kinetic study of enzymatic glycerolysis of refined sardine oil in tert-butanol as solvent catalyzed by the commercial lipase Lipozyme 435. The amount of tert-butanol added to create a monophasic system has been optimized based on liquid liquid equilibrium (LLE) data previously determined (Solaesa et al. 2013). This value has been compared with the amount of tert-butanol added to other glycerolysis systems. Results in terms of MAG and DAG yield have been also compared with literature data reported for different type of oils and relating to the high activity of the lipase for short and medium chain length fatty acid. 
First, external and internal mass transfer resistances have been analyzed in the heterogeneous system of the immobilized lipase. Mass transfer limitations can play an important role on the reaction rate. However, in most glycerolysis studies reported in the literature no mass transfer studies were performed.

Mathematical models are needed to predict and also optimize the industrial processes. However, not many works can be found in the literature dealing with kinetic modeling of glycerolysis. One of the first works was carried out by Moquin et al. (Moquin et al. 2005). In that work, the kinetics of the non-catalyzed glycerolysis of soybean oil in $\mathrm{SC}-\mathrm{CO}_{2}$ media were correlated by a sequence of reversible reactions taking into account parallel hydrolysis reaction. The same model was followed by Valerio et al. (Valério, Krüger, et al. 2009) in the kinetic study of solvent-free lipase-catalyzed glycerolysis of olive oil by Novozym 435 in Triton X-100 as surfactant. Although glycerolysis and hydrolysis reactions were proposed, no information about experimental FFA production and rate of change of glycerol is provided and only TAG, MAG and DAG concentrations were taken into account in the fitting procedure to obtain the kinetic parameters. The mechanism of glycerolysis and hydrolysis of pure POP (1,3-palmitin-2-olein) by Rhizopus arrhizus lipase was studied by Tan and Yin (Tan \& Yin 2005)by including different hydrolysis, esterification and isomerization of MAG and DAG. Cheirsilp et al. (Cheirsilp et al. 2007) proposed a PingPong $\mathrm{Bi} \mathrm{Bi}$ model focused on the kinetics of the hydrolysis and esterification steps involved in the glycerolysis reaction of palm oil in acetone/isooctane mixture $(3: 1 \mathrm{v} / \mathrm{v})$. Water was dissolved in glycerol (10\% w/v of water added to glycerol) and therefore high amount of water was present in the reaction media. Recently, Voll et al. (Voll et al. 2011) proposed a kinetic model based on the ordered-sequential Bi Bi mechanism for a lipase-catalyzed glycerolysis system of olive oil in tert-butanol as solvent. In that work, reaction products are expressed as total amount of MAG, DAG, TAG and FFA by weight percentage in a solvent-free basis composition and no experimental information about glycerol concentration rate of change was provided. Fiametti et al. (Fiametti et al. 2012) followed a similar model to the one proposed by Voll et al. (Voll et al. 2011) in the glycerolysis of olive oil by ultrasound irradiation. However, parameters are no provided in the open literature although they can be available upon requesting the authors. 
In this work a similar approach to the previously proposed by Moquin et al. (Moquin et al. 2005) has been followed. Kinetic parameters have been compared, when possible, with previous values reported in the literature. This model has been able to represent the concentration of all compounds involved in the glycerolysis system, TAG, DAG, MAG, FFA, glycerol and water.

\section{Experimental section}

\subsection{Materials}

Refined sardine oil was kindly provided by Industrias Afines S.L. (Spain) with a water content of $0.19 \pm 0.03 \%$. Glycerol was purchased from Sigma Aldrich with a purity of $\geq 99.5 \%$ and a water content of $0.18 \pm 0.04 \%$. Tert-butanol (TB) was purchased from Merck with a purity of $\geq 99 \%$ and a water content of $0.20 \pm 0.03 \%$. Products were stored over activated $3 \AA$ molecular sieves to keep them dried. The food grade lipase Lipozyme435 from Candida antarctica (immobilized on a macroporous hydrophobic acrylic resin) was kindly donated by Novozymes A/S (Bagsvaerd, Denmark). The water content of this lipase was $3.5 \pm 0.3 \%$ as determined by Karl-Fisher titration with a Mitsubishi CA-20 moisture meter in triplicate. According to Novozymes $A / S$, the specific activity of the lipase is $\geq 8000$ propyl laurate units/g. No additional water was added to the system. Therefore, water present in the reaction media comes only from reactants.

\subsection{Enzymatic glycerolysis of sardine oil}

Different vials containing a mixture of sardine oil, glycerol and TB were incubated at different temperatures from 303 to $333 \mathrm{~K}$ in a water bath with orbital agitation. Different mole ratios of substrates and enzyme dosages were also studied. The amount of TB added was fixed at a mass ratio of 1.5:1 (TB:substrates), calculated on the basis of previous studies on LLE (Solaesa et al. 2013). At selected time intervals (from five minutes up to eight hours), the reaction mixture was withdrawn and filtered through a microfilter $(0.45$ $\mu \mathrm{m}$, Sartorius RC) to stop the reaction by removing the lipase. All samples were stored at $-18{ }^{\circ} \mathrm{C}$ prior to analysis. 
The reusability of Lipozyme 435 in this process was tested by recycling the immobilized enzyme in 6 batches. After each run, the lipase was washed once with TB, and subsequently twice with hexane in order to eliminate remaining compounds. Afterwards, the lipase was dried at $303 \mathrm{~K}$ and stored in a desiccator under vacuum. No significant reduction in the enzyme activity was found. Anyway, fresh biocatalyst was used in each run.

Moreover, tert-butanol was evaporated under vacuum using a rotary evaporator (Heibolph VV2000) at $333 \mathrm{~K}$. This way, TB could be reused by using molecular sieves to eliminate the water content.

\subsection{Determination of reaction products}

The neutral lipid profile (TAG, DAG, MAG and FFA) was analyzed by a normal-phase high performance liquid chromatography (NP-HPLC). The chromatographic apparatus consisted of a HPLC system (Agilent 1200) formed by a quaternary pump and an auto-injector. The chromatographic separation of the compounds was carried out at room temperature with a Lichrospher Diol column $(5 \mu \mathrm{m}, 4 \mathrm{~mm} \times 250 \mathrm{~mm}$ ) and detection was performed in an evaporative light scattering detector (Agilent 1200 series) at $35^{\circ} \mathrm{C}$ and $0.35 \mathrm{MPa}$. Gradient elution was achieved by mobile phases A (isooctane) and B (methyl tert-butyl ether:acetic acid $=99.9: 0.1, v / v)$. The method and the calibration procedure were previously developed (Solaesa et al. 2014). The regioisomers of DAG and MAG could not be distinguished with the applied analytical procedure, therefore total amount of MAG and DAG is reported during the kinetic experiments.

The analysis of remaining glycerol was performed by using High-Temperature Gas Chromatography (HT-GC) system (HP 6890 Series GC System) equipped with a flame ionization detector (FID), a fused silica capillary column of $30 \mathrm{~m} \times 0.25 \mathrm{~mm}$ i.d. coated with a $0.25 \mu \mathrm{m}$ film thickness of $65 \%$ Phenyl Methylpolisiloxane (65HT) as stationary phase and Agilent Technologies 7683B Series automatic injector. The method and the calibration procedure were previously developed (Solaesa et al. 2013). 


\subsection{Kinetic modeling}

The overall glycerolysis reaction can be described by:

$\mathrm{TAG}+2 \mathrm{Gly} \rightleftarrows 3 \mathrm{MAG}$

However, glycerolysis reaction is believed to follow a two-step reaction. First, one molecule of glycerol reacts with one molecule of TAG to yield one molecule of DAG and another molecule of MAG. Reaction of one molecule of DAG with one molecule of glycerol can also take place to yield two molecules of MAG:

$\mathrm{TAG}+\mathrm{Gly} \underset{\mathrm{k}_{2}}{\stackrel{\mathrm{k}_{1}}{\rightleftarrows}} \mathrm{DAG}+\mathrm{MAG}$

$\mathrm{DAG}+\mathrm{Gly} \underset{\mathrm{k}_{4}}{\stackrel{\mathrm{k}_{3}}{\rightleftarrows}} 2 \mathrm{MAG}$

The breakdown of TAG due to reaction with MAG can also occur to produce two molecules of DAG (Moquin et al. 2005):

$\mathrm{TAG}+\mathrm{MAG} \underset{\mathrm{k}_{6}}{\stackrel{\mathrm{k}_{5}}{\rightleftarrows}} 2 \mathrm{DAG}$

Even in the presence of small amounts of water from glycerolysis reaction media, unwanted hydrolysis reactions must be considered:

$\mathrm{TAG}+\mathrm{H}_{2} \mathrm{O} \underset{\mathrm{k}_{8}}{\stackrel{\mathrm{k}_{7}}{\rightleftarrows}} \mathrm{DAG}+\mathrm{FFA}$

$\mathrm{DAG}+\mathrm{H}_{2} \mathrm{O} \underset{\mathrm{k}_{10}}{\stackrel{\mathrm{k}_{9}}{\rightleftarrows}} \mathrm{MAG}+\mathrm{FFA}$

$\mathrm{MAG}+\mathrm{H}_{2} \mathrm{O} \underset{\mathrm{k}_{12}}{\stackrel{\mathrm{k}_{11}}{\rightleftarrows}} \mathrm{GLY}+\mathrm{FFA}$

Kinetic models are needed to predict and simulate reactive processes. Formulating the mass balances equation for all the species of the reactive system a description of the 
concentration profile with time can obtained. This way, the global process can be optimized. The rate of change in concentration for each of the reaction components can then be described by the following differential rate equations:

$\frac{d n_{T A G} / n_{\text {total }}}{d t}=-k_{1} x_{T A G} x_{G l y}+k_{2} x_{D A G} x_{M A G}-k_{5} x_{T A G} x_{M A G}+$

$k_{6}\left(x_{D A G}\right)^{2}-k_{7} x_{T A G} x_{H_{2} O}+k_{8} x_{D A G} x_{F F A}$

$\frac{d n_{D A G} / n_{\text {total }}}{d t}=k_{1} x_{T A G} x_{G l y}-k_{2} x_{D A G} x_{M A G}-k_{3} x_{D A G} x_{G l y}+k_{4}\left(x_{M A G}\right)^{2}+$
$2 k_{5} x_{T A G} x_{M A G}-2 k_{6}\left(x_{D A G}\right)^{2}+k_{7} x_{T A G} x_{H_{2} O}-k_{8} x_{D A G} x_{F F A}-k_{9} x_{D A G} x_{H_{2} O}+k_{10} x_{M A G} x_{F F A}[9]$

$\frac{d n_{M A G} / n_{t o t a l}}{d t}=k_{1} x_{T A G} x_{G l y}-k_{2} x_{D A G} x_{M A G}+2 k_{3} x_{D A G} x_{G l y}-2 k_{4}\left(x_{M A G}\right)^{2}-$

$k_{5} x_{T A G} x_{M A G}+k_{6}\left(x_{D A G}\right)^{2}+k_{9} x_{D A G} x_{H_{2} O}-k_{10} x_{M A G} x_{F F A}-k_{11} x_{M A G} x_{H_{2} O}+k_{12} x_{M A G} x_{F F A}[10]$

$\frac{d n_{G l y} / n_{t o t a l}}{d t}=-k_{1} x_{T A G} x_{G l y}+k_{2} x_{D A G} x_{M A G}-k_{3} x_{D A G} x_{G l y}+$

$k_{4}\left(x_{M A G}\right)^{2}+k_{11} x_{M A G} x_{H_{2} O}-k_{12} x_{G l y} x_{F F A}$

$\frac{d n_{F F A} / n_{\text {total }}}{d t}=k_{7} x_{T A G} x_{H_{2} O}-k_{8} x_{D A G} x_{F F A}+k_{9} x_{D A G} x_{H_{2} O}-$

$k_{10} x_{M A G} x_{F F A}+k_{11} x_{M A G} x_{H_{2} O}-k_{12} x_{G l y} x_{F F A}$

$\frac{d n_{\mathrm{H}_{2} \mathrm{O}} / n_{\text {total }}}{d t}=-k_{7} x_{T A G} x_{\mathrm{H}_{2} \mathrm{O}}+k_{8} x_{D A G} x_{F F A}-k_{9} x_{D A G} x_{\mathrm{H}_{2} \mathrm{O}}+$

$k_{10} x_{M A G} x_{F F A}-k_{11} x_{M A G} x_{H_{2} O}+k_{12} x_{G l y} x_{F F A}$ 
As previously explained in the analytical procedure, the stereoisomers of DAG and MAG could not be distinguished and no difference could be made between them in the model. Concentrations of reaction products are expressed in solvent-free basis. TAG, DAG, MAG, FFA and glycerol concentrations have been experimentally determined. Water concentration could not be measured along reaction time. According to Moquin et al. (Moquin et al. 2005) it is possible to estimate the change in water concentration by subtracting the experimental FFA concentration from the initial water concentration since formation of one mole FFA requires one mole of water (Equations 5-7).

The rate constants for the six kinetic equations are obtained by solving the set of differential equations simultaneously. In this work, the differential equations were solved numerically with a fourth order Runge-Kutta method and by reducing the experimental kinetic data minimizing the following objective function (O.F.):

O.F. $=\frac{\sum_{\text {all samples }} \sum_{\mathrm{i}=1}^{\mathrm{n}}\left(\mathrm{x}_{\mathrm{i}, \exp }-\mathrm{x}_{\mathrm{i}, \mathrm{calc}}\right)^{2}}{\mathrm{n}_{\mathrm{samples}}} \cdot 100$

by using the Simplex-Nelder-Mead method. The subscript " $\mathrm{i}$ " refers to the different components in the glycerolysis system: TAG, DAG, MAG, FFA, glycerol and water. The subscripts "exp" and "calc" are referring to the experimental and calculated mole fraction of the different components for each experimental kinetic data $\left(\mathrm{n}_{\text {samples }}\right)$

The root-mean-square deviation ( $\mathrm{rmsd}$ ) has been also calculated to evaluate the quality of the fitting:

$r m s d=\sqrt{\frac{\sum_{i=1}^{N O B S}\left(w_{i}^{\text {exp }}-w_{i}^{\text {calc }}\right)^{2}}{N O B S}}$

where NOBS is the total number of kinetic data for all the kinetic experiments and $w_{i}^{\text {exp }}$ and $w_{i}^{\text {calc }}$ the experimental and calculated weight fraction for all the reaction compounds. 


\section{Results and discussion}

\subsection{Mass transfer analysis}

External and intraparticle mass transfer resistance can influence the observed reaction rate in heterogeneous catalyzed processes such as immobilized lipase biocatalysis. Previous to the study of the effect of some kinetic variables, mass transfer was analyzed.

In this work, tert-butanol has been used as organic solvent to provide an environment where oil and glycerol can interact since both reactants are completely immiscible. Tertbutanol helps to create a homogeneous phase but also decreases the viscosity of the reaction medium since both reactants are highly viscous, especially glycerol (Table 3.1).

Table 3.1. Initial rate of MAG formation as a function of orbital speed ( $\mathrm{T}=303.15 \mathrm{~K}, 2.5 \%$ enzyme loading based on substrate weight, $M R=3: 1$ ). Viscosity of reaction compounds.

\begin{tabular}{lcc} 
Orbital speed, $\mathrm{rpm}$ & $\mathrm{r}_{\mathrm{o}}, \mathrm{mmol} \cdot \mathrm{L}^{-1} \cdot \mathrm{min}^{-1}$ \\
120 & $0.7 \pm 0.1$ & $0.8 \pm 0.2$ \\
170 & $0.8 \pm 0.1$ & $\mathbf{3 2 3 \mathrm { K }}$ \\
200 & \multicolumn{2}{c}{ Viscosity, $\mathrm{mPa} \cdot \mathrm{s}$} \\
& $303 \mathrm{~K}$ & 142 \\
Compound & 612 & $20-30$ \\
Glycerol (Sengwa et al. 2010) & $60-90 *$ & 1.421 \\
Fish oil (Young 1986) & 3.392 & \\
Tert-butanol (Chowdhury \& Saleh 2014) & &
\end{tabular}

*: value at $298.15 \mathrm{~K}$

To evaluate the external mass-transfer resistance glycerolysis reaction was carried out at different orbital speeds, from 120 to $200 \mathrm{rpm}$, while keeping constant the rest of reaction conditions. The results are presented in Table 3.1. From these results it can be concluded that there is no increase in the initial reaction rate of MAG formation in the speed range studied in this work. This result was expected since external diffusion does not usually 
control the overall rate unless the agitation speed is very low or the reaction mixture is very viscous (Sanz et al. 2002). This way, tert-butanol helps to decrease the viscosity of the reaction medium since its viscosity is 100 time less than the viscosity of glycerol (Table 3.1) resulting in a low external mass transfer resistance and acts as an inert carrier for reactants to the active site of the enzyme. Hence, $170 \mathrm{rpm}$ was chosen for all glycerolysis reactions.

Slow intraparticle diffusion may reduce the overall reaction rate, especially if the reactant molecules are large (Helfferich 1962) and have a low mobility in the lipase support. Chesterfield et al. (Chesterfield et al. 2012) analyzed the relative magnitude of the external liquid mass transfer resistance to the combined internal resistances (intraparticle diffusion and reaction resistances) in the ethanolysis of waste cooking oil by using Novozym 435 by plotting the reciprocal initial reaction rate $\left(1 / r_{0}\right)$ as a function of inverse lipase loading $(1 / \mathrm{m})$. This plot should be a straight line, which slope is proportional to the combined internal resistances, and the intercept proportional to the interphase mass transfer resistance. Figure 3.1 illustrates this linear dependence in the glycerolysis of sardine oil. The linear fit proves that the rate controlling step is the combined internal resistances since the intercept can be considered negligible.

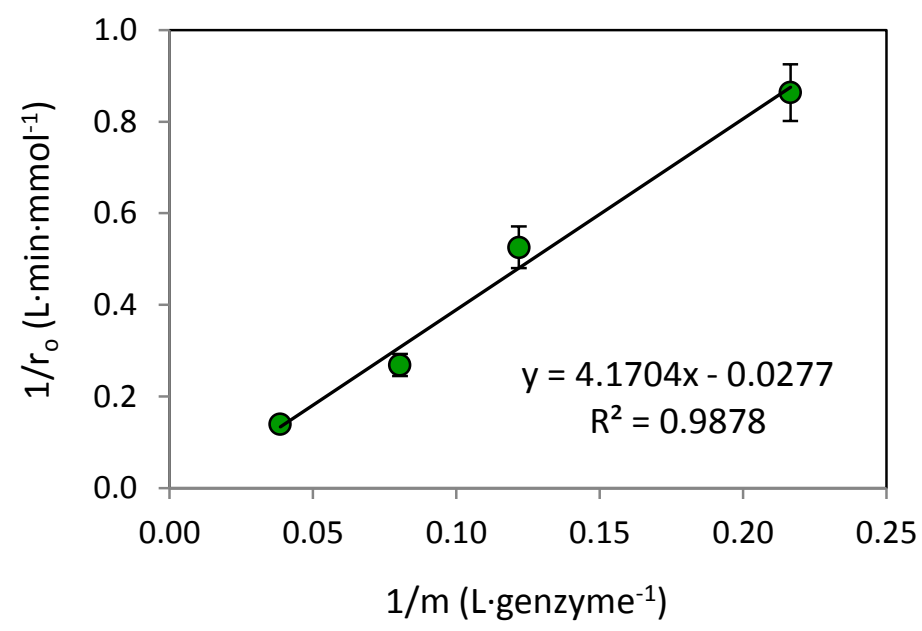

Figure 3.1. Effect of catalyst loading on initial reaction rate of $M A G$ formation $(T=323 \mathrm{~K}$, $M R=3: 1)$ 
To evaluate the intraparticle diffusion effect, the lipase Lipozyme 435 was separated into two fractions by a $400 \mu \mathrm{m}$ sieve (46 wt \% of Lipozyme 435 particles with $\varphi_{\mathrm{p}}>400 \mu \mathrm{m}$ ). Kinetic experiments were carried out with each of the fractions obtained and compared with the results obtained with the unsieved lipase. Figure $\mathbf{3 . 2}$ shows that initial reaction rate of MAG formation increased by decreasing the particle size of Lipozyme 435 what might indicate internal mass transfer limitation for the larger particles, although the same MAG yield is achieved at long reaction time. A significant pore diffusion resistance was also found by Chesterfield et al. (Chesterfield et al. 2012) in the ethanolysis study with Novozym 435 (technical grade of Candida antartica).

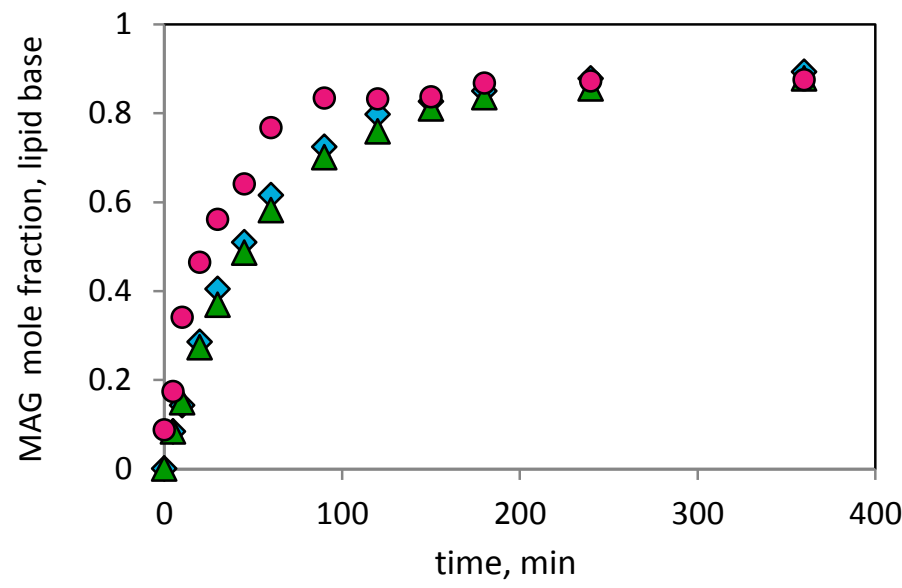

Figure 3.2. Effect of particle size $(\bullet) d_{p}<400 \mu \mathrm{m} ;(\bullet)$ unsieved lipase; $(\Delta) d_{p}>400 \mu \mathrm{m}$ on MAG formation reaction: $\mathrm{T}=323 \mathrm{~K}, 5 \mathrm{wt} \%$ Lipozyme 435 loading, MR =3:1. Standard uncertainty $\mathrm{u}$ (mole fraction) $=0.02$.

The experimental Thiele modulus, $\phi_{\exp }$, was calculated to evaluate intraparticle resistance (Dong et al. 2010):

$\phi_{\text {exp }}=\left(\frac{d_{p}}{6}\right)^{2} \frac{r_{\text {exp }, \text { substrate }}}{D_{\text {eff }} C_{\text {substrate }, o}}$

$d_{p}$ is the mean particle diameter of Lipozyme $435\left(d_{p}=383 \mu \mathrm{m}\right.$, (Chesterfield et al. 2012)). The effective diffusivity, $D_{\text {eff, }}$ was evaluated through (Fogler 1999):

$D_{\text {eff }}=\frac{D_{\text {substrate-solvent }} \varepsilon_{p} \sigma}{\tau}$ 
where $\varepsilon_{p}, \tau$ and $\sigma$ are Lipozyme 435 porosity, tortuosity and constriction factor. These values were taken from Chesterfield (Chesterfield et al. 2012) for Novozym $435\left(\varepsilon_{p}=0.5\right.$, $\tau=6$ and $\sigma=1$ ). $D_{\text {substrate-solvent }}$ is the molecular diffusivity of the reactants (glycerol and fish oil) in the reaction medium (tert-butanol in this work) and it was estimated using the Wilke Chang equation (Reid et al. 1987):

$D_{\text {substrate-solvent }}=\frac{7 \cdot 4 \cdot 10^{-8} T\left(M_{\text {substrate }} \psi_{\text {solvent }}\right)}{\eta_{\text {solvent }} V_{\text {substrate }}^{0.6}}$

where $D_{\text {substrate-solvent }}$ is the diffusion coefficient of the substrate in the solvent $\left(\mathrm{cm}^{2} \cdot \mathrm{s}^{-1}\right)$, $M_{\text {substrate }}$ is the molecular weight of solvent $(\mathrm{g} / \mathrm{mol}), T$ is the temperature $(K), \eta_{\text {solvent }}$ is the viscosity of the solvent, $\mathrm{CP}, \mathrm{V}_{\text {sustrate }}$ is the molar volume of the substrate at its normal boiling temperature, $\mathrm{cm}^{3} / \mathrm{mol}$ and $\psi$ the association factor of solvent (dimensionless, $\psi=1$ for non-associated compounds). Parameters values used in the calculation of $\phi$ are listed in Table 3.2. Molar volumes at the normal boiling point have been estimated by the Tyn and Calus method (Reid et al. 1987):

$V=0.285 V_{c}^{1.048}$

where $V_{c}$ is the critical volume in $\mathrm{cm}^{3} / \mathrm{mol}$. $V_{c}$ for glycerol was $255 \mathrm{~cm}^{3} / \mathrm{mol}$ (Reid et al. 1987). No data of $V_{c}$ for fish oil was found in the literature and the corresponding estimated value for triolein $\left(V_{c}=3235.65 \mathrm{~cm}^{3} / \mathrm{mol}\right)$ was used (Olivares-Carrillo et al. 2014). $\Phi$ has been evaluated for both substrates, glycerol and sardine oil at $323 \mathrm{~K}$ for $\mathrm{r}_{\text {exp,glycerol }}=$ $0.0173 \mathrm{mmol} \cdot \mathrm{L}^{-1} \cdot \mathrm{s}^{-1}, \mathrm{C}_{\text {glycerol,o }}=47.5 \mathrm{mmol} \cdot \mathrm{L}^{-1} \mathrm{r}_{\text {exp }, \text { fish oil }}=0.023 \mathrm{mmol} \cdot \mathrm{L}^{-1} \cdot \mathrm{s}^{-1} \mathrm{C}_{\text {fish oil,o }}=47.5$ $\mathrm{mmol} \cdot \mathrm{L}^{-1}$.

Table 3.2. Thiele module and parameters values used in its calculation

\begin{tabular}{ll} 
Parameter & Value \\
$V_{\text {glycerol }}$ & $94.82 \mathrm{~cm}^{3} \cdot \mathrm{mol}^{-1}$ \\
$\mathrm{~V}_{\text {fish oil = triolein }}$ & $1359.20 \mathrm{~cm}^{3} \cdot \mathrm{mol}^{-1}$ \\
\hline$\Phi_{\text {glycerol }}=0.019 \pm 0.004<0.3$ & $\Phi_{\text {fish oil }}=0.35 \pm 0.09 \sim 0.3$ \\
\hline
\end{tabular}


According to Bailey (Bailey 1986), when $\Phi$ is sufficiently large $(\Phi \geq 3)$, diffusion of substrate is slow relative to its consumption. When $\Phi<0.3$, the limiting rate process is the chemical reaction. $\Phi$ for diffusion of glycerol in the reaction medium was found to be $1.9 \cdot 10^{-2}$, however a value of 0.36 was obtained for diffusion of fish oil in tert-butanol, probably due to the bigger size of oil molecules that could lead to more diffusional limitation (Table 3.2). In any case the value of $\Phi$ is close to the limit of 0.3 and the observed rate can be considered kinetically controlled. Based on the $\Phi$ values the lipase was used in its commercially available size without sieving for further kinetic experiments.

Yang et al. (Yang et al. 2005a) studied the effect of the load of Novozym 435 on the glycerolysis of sunflower oil. They found that an enzyme loading of more than $10 \%$ resulted in only a small increase in MAG yield; therefore they suggested that $10-15 \%$ of enzyme load was enough to obtain maximum reaction performance. Moreover, other authors as Valerio et al. and Fiametti et al. (Fiametti et al. 2012; Valério, Krüger, et al. 2009) showed that high enzyme concentrations could lead to the formation of aggregates, making the enzyme active site not available to the substrates. Based on this finding and the results shown in Figure $\mathbf{3 . 1}$ further glycerolysis kinetics have been performed with a 10 wt\% of Lipozyme 435 based on reactants weight.

\subsection{Glycerolysis reaction system}

The presence of a catalyst is necessary since it has been proved in the literature (Krüger et al. 2010) that under $70^{\circ} \mathrm{C}$ the observed reaction rate without a catalyst is nearly zero. Figure $3 c$ shows a typical glycerolysis profile of fish oil at mole ratio of glycerol:sardine oil of $3: 1$ at $323 \mathrm{~K}$ with $10 \%$ of lipase loading in tert-butanol (68 \% of tert-butanol). The main reaction product at the above conditions is MAG (around $51 \%$ mole percentage), but DAG and FFA production is also observed although mole percentage was around $3 \%$ for both compounds. Initial water content in the reaction media is less than $1 \%$ in weight but it is nearly $10 \%$ of mole content of water in the reaction media; therefore FFA production can be observed. TAG consumption is nearly complete with a mole percentage at equilibrium conditions lower than $2 \%$. 


\section{$\underline{\text { Effect of reactant mole ratio }}$}

Initial mole reactant ratio (MR) was varied between 1 and 9. Figures 3.3a-3.3d show the glycerolysis product profile expressed in mole fraction in a solvent-free basis. Reaction rate formation of MAG was always higher than that of DAG and FFA. The presence of a solvent, tert-butanol, helps both reactants to diffuse to the active site of the enzyme and MAG formation is favored. Valerio et al. (Valério, Krüger, et al. 2009) studied the kinetics of glycerolysis of olive oil in a surfactant system (with Triton X-100 as surfactant) as an alternative to the use of organic solvents and found that DAG initial reaction rate was higher than that of MAG even with an excess of glycerol (MR=9:1). This behavior could be due to mass transfer limitations that can be compared to a situation of low glycerol concentration in the reaction medium.

The optimal MR glycerol:oil must consider the MAG yield as well as the excess of glycerol employed in the glycerolysis reaction. Equilibrium yield of MAG has been calculated as:

EquilibriumMAGyield $(\%)=\frac{\text { MolesofMAGintheequilibrium }}{\text { Initialmolesof } T A G \cdot 3} \cdot 100$

Figure 3.4 shows that MAG equilibrium yield remains practically constant at MR greater than 5:1. A similar behavior was observed by Chesterfield et al. (Chesterfield et al. 2012)in the ethanolysis of waste cottonseed cooking oil by Novozym 435. These authors proposed the following relationship for the equilibrium yield:

EquilibriumMAGyield $(\%)=\frac{a}{1+\exp \left(\frac{R M_{O}-R M}{b}\right)}$

Non-linear regression was performed by using Marquardt algorithm (Statgraphics) obtaining $\mathrm{a}=89.285$ defined as the limiting normalized MAG equilibrium (Chesterfield et al. 2012), $b=0.922$ and $\mathrm{RM}_{\mathrm{o}}=1.35$ with $\mathrm{r}^{2}=0.999$. McNeil and Yamane (McNeill \& Yamane 1991) also found that MAG equilibrium yield was also independent of the glycerol:oil mole ratio from mole ratio greater than 5:1. 

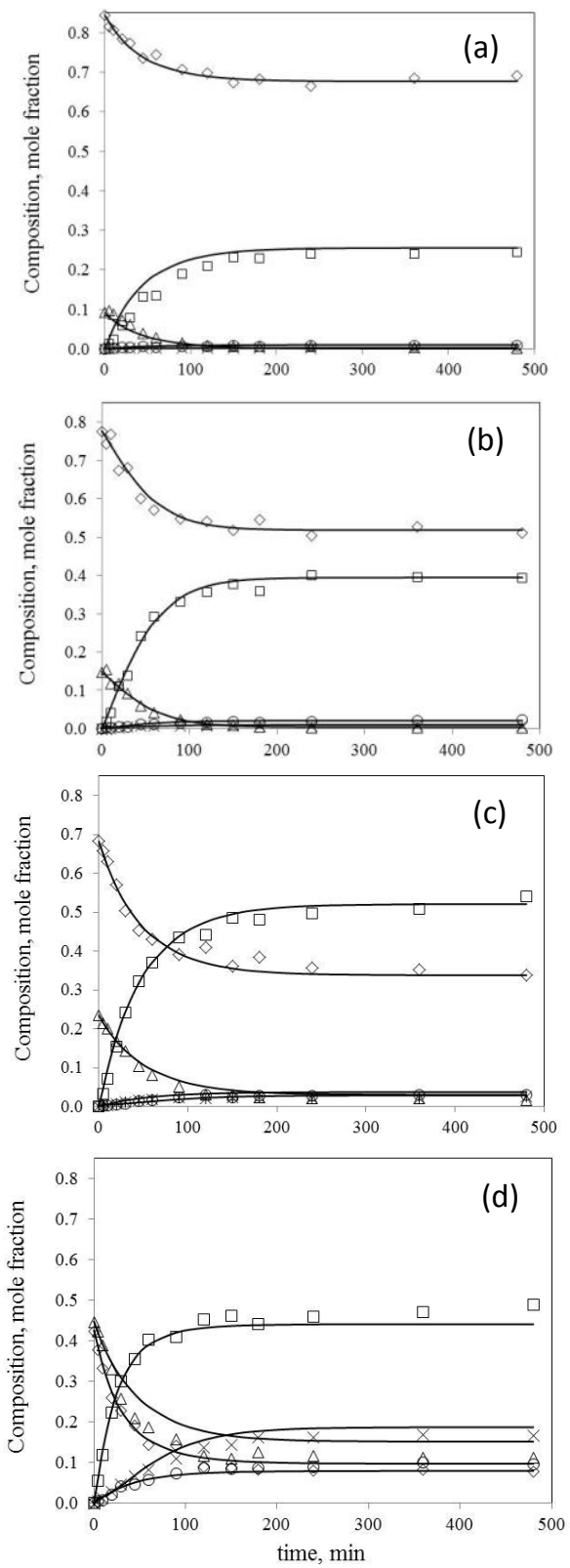

Figure 3.3. Time course for glycerolysis reaction at different mole ratio (MR): (a) 9:1, (b) 5:1, (c) 3:1 (d) 1:1; 323 K, 10 wt \% Lipozyme 435 loading; $\square$ MAG o FFA $\Delta$ TAG $\diamond$ glycerol $\times$ DAG. Continuous lines correspond to the model proposed in this work. Standard uncertainty $\mathrm{u}$ (mole fraction) $=0.02$. 


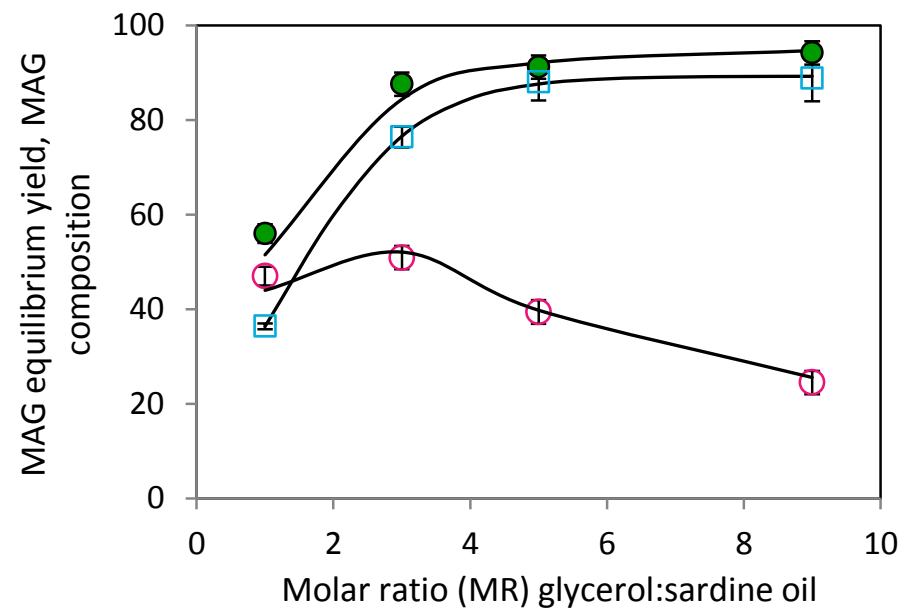

Figure 3.4. MAG equilibrium yield $(\square)$ as a function of initial mole ratio (MR) glycerol:oil. Continuous line corresponds to equation 20. MAG composition as mole percentage in a solvent and glycerol free-basis $(\bullet)$ and in a solvent free-basis $(\circ)$. Continuous lines correspond to the equilibrium composition obtained with the model proposed in this work.

To take into account the excess of glycerol employed, Figure $\mathbf{3 . 4}$ also shows the MAG composition (expressed as mole percentage) in a solvent-free basis and in solvent and glycerol-free basis. In a lipid basis (no glycerol) by increasing the MR, MAG content increases sharply from a MR of 1:1 to $3: 1$ and then MAG content slightly increases in the lipid fraction. In a solvent-free basis, when glycerol is considered in the global composition, a maximum is observed in the MAG content at a MR of $3: 1$, due to the excess of glycerol employed that is not consumed.

Table 3.3 summarizes glycerides equilibrium composition found in this work, as well as for other glycerolysis systems found in the literature that use tert-butanol as solvent and immobilized Candida antarctica as biocatalyst. Results are expressed in weight percentage in a lipid basis, since in most studies composition is usually expressed in this way. Although different lipase loadings can be observed in Table 3.3, data listed in this table correspond in most cases to equilibrium conditions and comparison of MAG yield could be established. Table 3.3 shows different results in terms of MAG and DAG yield even at the 
same initial MR (as it will be explained in section 3.2.2, the effect of temperature in MAG equilibrium yield is not very important). For instance at MR glycerol:oil of $4: 1$, MAG percentage in a lipid basis ranges from $70 \%$ for sunflower oil to $91 \%$ for tuna oil. Regarding the type of oil, fish oils present higher MAG yield than vegetable oils. According to the shape and properties of the respective scissile fatty acid binding sites of Candida antarctica lipase, in the literature it has been reported that this lipase has high activity for short and medium chain length fatty acids (Pleiss et al. 1998). Table 3.4 presents the fatty acid composition of the oils listed in Table 3.3. It can be observed that fish oils have the highest content of medium chain length fatty acids as C14:0, C16:0 and C16:1. Based on these results, a relationship between fatty acid specificity of Candida antarctica lipase and MAG yield for the different type of oils could be established. From Table 3.3, it can also be observed that the amount of tert-butanol added to the system is different ranging from $45 \%$ to $80 \%$. Tert-butanol helps to create a homogeneous reaction system, avoiding mass transfer limitations. Figure $\mathbf{3 . 5}$ represents the binodal curve for the ternary system glycerol + sardine oil + tert-butanol at 303.15 and $323.15 \mathrm{~K}$ (Solaesa et al. 2013). In this graph the initial composition, expressed in weight fraction $\left(w_{\text {glycerol }}, w_{\text {oil }}, w_{\text {tert-butanol }}\right)$, of the different glycerolysis systems listed in Table $\mathbf{3 . 3}$ have been also represented. Although binodal curves could be different for the oils compared in this work, miscibility region is expected to be of the same order. From this graph it can be observed that in most glycerolysis systems a homogenous phase is obtained by adding enough amount of tertbutanol; that is, initial glycerolysis composition lies in the one phase region. However in both glycerolysis studies for olive oil (Krüger et al. 2010; Voll et al. 2011), around $45 \%$ weight percentage of tert-butanol is added to system and this amount seems to be not enough to create a homogenous phase. This could also explain the low MAG yield obtained in these studies (around $65 \%$ ) compared to other systems. In any case, MAG and DAG formed during the glycerolysis could probably act as emulsifier avoiding somehow mass transfer limitations. Nonetheless, in these cases, mass transfer limitations probably would take place at the beginning of the process. Figure $\mathbf{3 . 6}$ shows initial reaction rates as a function of initial MR glycerol:oil . It can be observed an increase of initial reaction rate for MAG and glycerol with MR up to 3. At MR greater than 3, a decay is observed. This could be due to glycerol inhibition of lipase-catalyzed reaction at high MR glycerol:oil. 
Initial reaction rates for TAG consumption and DAG and FFA production continuously decreased by increasing the MR. According to Figures 3.3 and 3.6, DAG production is favored by restricting the glycerol amount in the reaction medium. Similar findings were observed in other glycerolysis studies (Krüger et al. 2010). Krüger et al. (Krüger et al. 2010) reported lower values for the initial reaction rates in the glycerolysis of olive oil at $328 \mathrm{~K}$, 15 wt\% of Novozyme and tert-butanol to substrate volume ratio of 1:1 (approximately 45 wt\% of tert-butanol, see Table 3.3). These authors obtained initial reaction rates of 2.136, 1.301 and $1.293 \mathrm{mmol} / \mathrm{min}$ at $\mathrm{MR}$ of $3: 1,6: 1$ and 9:1, respectively. The low values obtained by Kruger et al. (Krüger et al. 2010) compared to the values obtained in this work (Figure 3.6) could be explained by assuming higher mass transfer limitations at the beginning of the process due to uncompleted miscibility of reactants (see Figure 3.5). These authors also reported initial reaction rates for DAG production at the conditions previously detailed of $0.375,0.221$ and $0.208 \mathrm{mmol} / \mathrm{min}$ at $\mathrm{MR}$ of $3: 1,6: 1$ and 9:1, respectively. These values are of the same order as the one obtained in this work (Figure 3.6). Finally, it has been chosen an optimal mole ratio of $3: 1$, taking into account the different effects of the amount of glycerol on glycerolysis kinetics.

In a previous work, tert-pentanol was used as organic solvent (Solaesa, Sanz, Falkeborg, et al. 2016). In that work, the effect of glycerol:oil mole ratio on MAG equilibrium yield and reaction rate was studied at three different values, 1:1, 3:1 and 5:1. MAG yield up to $90 \%$ was reached at a mole ratio of 3:1, slightly higher than the value found when using tertbutanol as solvent (84\%). No differences in MAG yield at higher mole ratio could be observed for both tert-alcohols. Additionally, higher initial reaction rates were observed when using tert-pentanol as solvent. These findings could be related with the polarity of the solvents $\left(\log \mathrm{P}_{\mathrm{TB}}=0.35\right.$ and $\left.\log \mathrm{P}_{\mathrm{TP}}=0.85\right)$ and the hydrophobicity of the support of Lipozyme 435. Due to the higher hydrophobicity of tert-pentanol the diffusion of reactants to the active site of the enzyme could be favored. 
Table 3.3. Equilibrium composition of glycerolysis reaction found in this work and for other glycerolysis systems found in the literature that use tert-butanol as solvent and immobilized Candida antarctica as biocatalyst.

\begin{tabular}{|c|c|c|c|c|c|c|c|c|c|}
\hline Oil & $\mathrm{T}, \mathrm{K}$ & $\% E$ & MR & $\%$ TB & $\%$ MAG & $\%$ DAG & $\%$ TAG & $\%$ FFA & Reference \\
\hline \multirow[t]{4}{*}{ Sardine } & \multirow[t]{4}{*}{323} & \multirow[t]{4}{*}{10} & $1: 1$ & 63 & $43.0 \pm 1.5$ & $25.8 \pm 1.9$ & $24.6 \pm 1.5$ & $6.6 \pm 1.1$ & \multirow[t]{4}{*}{ This work } \\
\hline & & & $3: 1$ & 68 & $83.3 \pm 2.1$ & $6.9 \pm 1.1$ & $5.9 \pm 1.0$ & $3.8 \pm 1.1$ & \\
\hline & & & $5: 1$ & 68 & $89.1 \pm 1.8$ & $3.7 \pm 0.8$ & $3.0 \pm 1.0$ & $4.0 \pm 1.3$ & \\
\hline & & & $9: 1$ & 74 & $92.9 \pm 1.5$ & $2.0 \pm 0.7$ & $2.4 \pm 0.8$ & $2.8 \pm 1.1$ & \\
\hline Sunflower & 323 & 21 & $4: 1$ & 73 & 71.3 & 22.1 & 0.6 & 5.2 & (Damstrup et al. 2005) \\
\hline Sunflower ${ }^{a}$ & 313 & 15 & $4.5: 1$ & 60 & 70 & 25 & 1 & 4 & (Yang et al. 2005a) \\
\hline Tuna & 318 & 15 & $4: 1$ & 58.6 & 90.8 & 2.5 & 5.5 & 1.2 & (Pawongrat et al. 2008) \\
\hline Camelia & 323 & 5 & $4: 1$ & 66 & $74.1 \pm 2.7$ & $24.6 \pm 0.1$ & $1.3 \pm 0.1$ & $--^{b}$ & (Zeng et al. 2010) \\
\hline \multirow[t]{5}{*}{ Olive $^{a, c}$} & 328 & 10 & $6: 1$ & 45 & 67 & 17 & 12 & 4 & \multirow[t]{5}{*}{ (Krüger et al. 2010) } \\
\hline & 328 & 2.5 & $3: 1$ & 45 & 34 & 15 & 50 & 1 & \\
\hline & 328 & 2.5 & $3: 1$ & 80 & 42 & 19 & 36 & 3 & \\
\hline & 343 & 2.5 & $9: 1$ & 45 & 53 & 11 & 33 & 3 & \\
\hline & 343 & 2.5 & $9: 1$ & 80 & 60 & 14 & 23 & 3 & \\
\hline Olive $^{a}$ & 328 & 10 & $6: 1$ & 45 & $\sim 62$ & $\sim 19$ & $\sim 15$ & $\sim 4$ & (Voll et al. 2011) \\
\hline
\end{tabular}

(a) Graphical lecture

(b) No reference to FFA formation

(c) Data at 720 min of reaction time. 
Table 3.4. Composition of medium chain length fatty acids in the oils used in the glycerolysis systems listed in Table 3.3.

\begin{tabular}{lccc|c} 
Oil & \multicolumn{2}{c|}{ Medium chain length fatty acids (\%) } & \\
& C14:0 & C16:0 & C16:1 & Reference \\
Sardine & $12.4 \pm 0.4$ & $22.8 \pm 0.2$ & $12.5 \pm 0.1$ & This work \\
Tuna & 4.2 & 30.6 & 4.7 & (Pawongrat et al. 2007) \\
Sunflower & 0.1 & 6.7 & 0.2 & $\begin{array}{c}\text { (Damstrup et al. 2005) } \\
\text { (Krüger et al. 2010; Voll et }\end{array}$ \\
Olive & $0.1-1.2$ & $7.0-16.0$ & - & al. 2011) \\
Camellia & - & 8.2 & - & (Zeng et al. 2010) \\
\hline
\end{tabular}

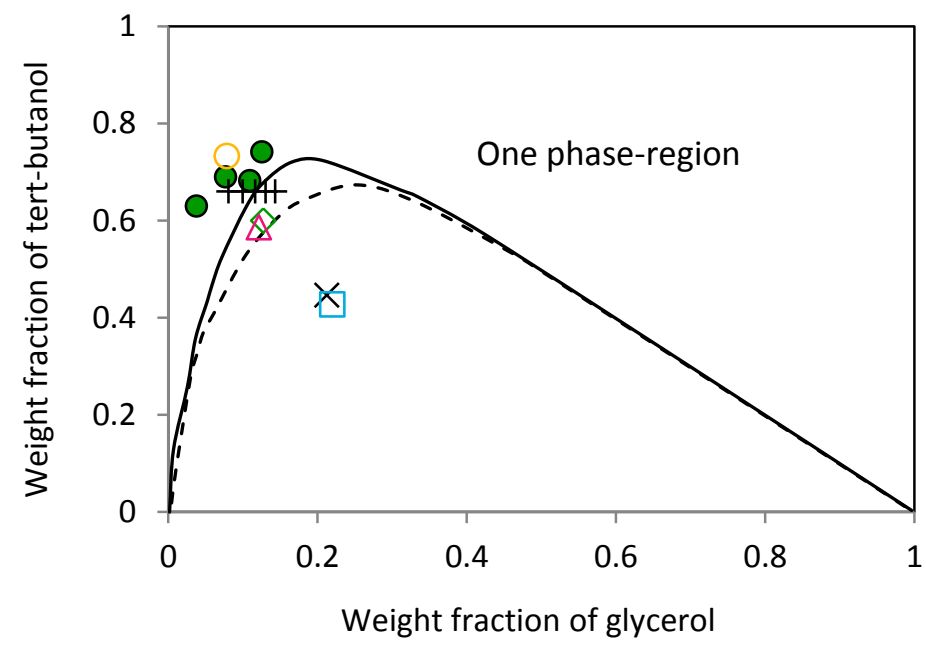

Figure 3.5. Binodal curve of the ternary system glycerol + fish oil + tert-butanol at $303.15 \mathrm{~K}$ $(-)$ and $323.15 \mathrm{~K}(---)$. Initial composition of glycerolysis reaction in tert-butanol medium: this work, $\bigcirc$ Sunflower oil (Damstrup et al. 2005), $>$ Sunflower oil (Yang et al. 2005a), $\Delta$ Tuna oil (Pawongrat et al. 2008), + Camellia oil (Zeng et al. 2010), $\square$ Olive oil (Voll et al. 2011), $\times$ Olive oil (Krüger et al. 2010) 


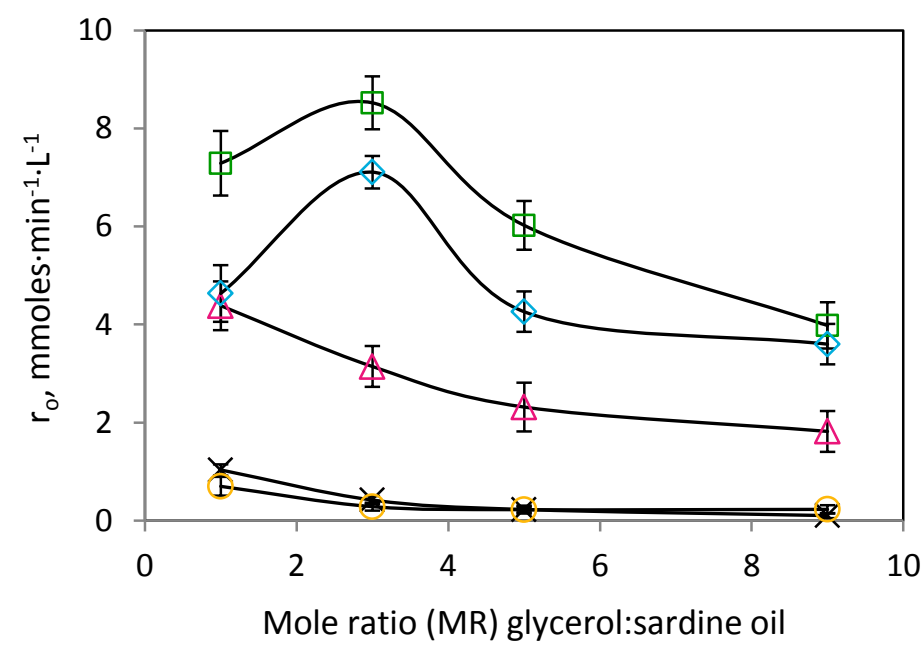

Figure 3.6. Initial reaction rate as a function of initial mole ratio glycerol:oil ( $323 \mathrm{~K}$, 10 wt \% Lipozyme 435 loading): $\square$ MAG $\circ$ FFA $\triangle$ TAG $\diamond$ glycerol $\times$ DAG.

Table 3.5 lists the kinetic parameters $\left(k_{1}-k_{12}\right)$ of the model proposed in this work and the values of the objective function for the different kinetic experiments. At the different mole ratios studied in this work, the rate of the second step, $k_{3}$, (DAG to produce MAG) is larger than the first step, $k_{1}$, (TAG to DAG). Therefore, the initial breakdown of TAG is slower and it is the rate-limiting step. Formation of MAG due to equation 3 has been found negligible $\left(k_{6}=0\right)$, as well as hydrolysis of TAG, $k_{7}$. From the values of model parameters, it can be concluded that esterification rates for glycerol, $k_{12}, M A G, k_{10}$, and DAG, $k_{8}$, with free fatty acids follows the sequence $k_{8}<k_{10}<k_{12}$. This result comes from steric hindrance of these groups (Tan \& Yin 2005). This tendency has been also observed by Moquin et al. (Moquin et al. 2005) in their kinetic modeling of glycerolysis of soybean oil in supercritical carbon dioxide media and Voll et al. (Voll et al. 2011) when using an ordered-sequential $\mathrm{Bi} \mathrm{Bi}$ mechanism in the glycerolysis of olive oil in tert-butanol. However, Valerio et al. (Valério, Krüger, et al. 2009) reported the following order $k_{10}<<$ $\mathrm{k}_{12}<\mathrm{k}_{8}$ for a solvent-free Novozym 435 catalyzed glycerolysis of olive oil in a surfactant system. Based on the values of model parameters (Table 3.5) the production rate of MAG by esterification of glycerol, $\mathrm{k}_{12}$, is of the same order as the production rate by hydrolysis reaction of $D A G, k_{9}$. 
Table 3.5. Calculated kinetic model parameters at different glycerol:oil mole ratio $(T=$ $323.15 \mathrm{~K}, 10 \mathrm{wt} \%$ Lipozyme 435 based on substrate weight). Objective function and root mean square deviation (wt \%) for glycerolysis products.

\begin{tabular}{lcccc} 
Model & \multicolumn{5}{c}{ Mole ratio } \\
parameter & $1: 1$ & $3: 1$ & $5: 1$ & $9: 1$ \\
\hline$k_{1}$ & 0.0350 & 0.0264 & 0.0263 & 0.0285 \\
$k_{2}$ & 0.0023 & 0.0248 & 0.0292 & 0.0276 \\
$k_{3}$ & 0.7638 & 0.9167 & 0.9411 & 0.9411 \\
$k_{4}$ & 0.0749 & 0.0400 & 0.0348 & 0.0338 \\
$k_{5}$ & 0.0108 & 0.0096 & 0.0047 & -- \\
$k_{6}$ & -- & -- & -- & -- \\
$k_{7}$ & -- & -- & -- & -- \\
$k_{8}$ & 0.0711 & 0.0046 & -- & -- \\
$k_{9}$ & 1.8168 & 1.9017 & 1.9022 & 1.9019 \\
$k_{10}$ & 0.4052 & 0.0234 & 0.0202 & 0.0209 \\
$k_{11}$ & 0.5853 & 0.8911 & 0.8942 & 0.8983 \\
$k_{12}$ & 1.9714 & 1.8052 & 1.8033 & 1.8008 \\
\hline O.F. & 0.0019 & 0.0013 & 0.0009 & 0.0012 \\
\hline Root mean squared deviation (wt \%) & & \\
\hline TAG & 4.7 & 3.4 & 5.6 & 4.6 \\
DAG & 1.0 & 0.9 & 0.4 & 0.2 \\
MAG & 3.8 & 2.7 & 5.8 & 5.6 \\
FFA & 1.1 & 0.7 & 0.5 & 0.3 \\
Glycerol & 0.4 & 1.3 & 1.7 & 2.2 \\
Water & 0.2 & 0.3 & 0.1 & 0.3 \\
\hline
\end{tabular}

It has been described that MAG yield is favored at mole ratios greater than the stoichiometry (2:1). Voll et al. (Voll et al. 2011) proposed as most obvious hypothesis that the excess of glycerol could react with DAG to produce 2 moles of MAG (see equations 12). This fact is reflected in the value of $k_{3}$ parameter as a function of mole ratio. $k_{3}$ increased sharply from MR of 1 to 3 and then remains constant, similar to MAG equilibrium yield dependence with MR (Figure 4). On the contrary, Voll et al. (Voll et al. 2011) found in their kinetic model that the kinetic parameter for the DAG to MAG step was negligible and attributed the increase in MAG yield with an excess of glycerol to the 
hydrolysis/esterification steps (equations 4-6). In our study, the kinetic parameter of the hydrolysis of TAG, $k_{7}$, has been found negligible; although, parameter model for DAG hydrolysis, $k_{9}$, and esterification of FFA formed, $k_{12}$, are of considerable importance.

The continuous lines in Figure $\mathbf{3 . 3}$ correspond to the model proposed in this work. Good agreement can be observed between experimental and calculated product concentrations.

\section{Effect of temperature}

Different kinetic experiments were carried out at different reaction temperatures, from 303 to $333 \mathrm{~K}$, with an enzyme concentration of $10 \mathrm{wt} \%$ (based on substrates weight) and at the previous identified optimal glycerol:sardine oil ratio of 3:1. Figure 3.7 shows the experimental kinetic data at the different reaction temperatures. Reaction rates of MAG, DAG and FFA formation as well as TAG and glycerol consumption increase by increasing reaction temperature (initial reaction rates values at 303, 313, 323 and $333 \mathrm{~K}$ for MAG production were found $2.69,4.26,8.16$ and $9.69 \mathrm{mmol} \cdot \mathrm{min}^{-1} \cdot \mathrm{L}^{-1}$ respectively). An increase of reaction rate by 3.0 times is obtained from 303 to $323 \mathrm{~K}$. Guo and Xu (Guo \& Xu 2006) found an increase by 2.2 times from 303 to 323 K, and Krüger et al. (Krüger et al. 2010) by 1.8 times from 313 to $343 \mathrm{~K}$. An optimal working temperature in the range of $313-338 \mathrm{~K}$ has been reported for Novozyme 435 (Valério, Krüger, et al. 2009). From Figure 3.7 it can be also observed that reaction temperature has a slight effect on equilibrium product concentrations. This behavior with temperature has been also observed for other transesterification reactions (Bucio et al. 2015; Krüger et al. 2010).

Table 3.6 lists the kinetic parameters $\left(k_{1}-k_{12}\right)$ of the semi-empirical model and the values of the objective function at the different temperatures essayed in this work. The continuous lines in Figure 3.7 correspond to the model. Good agreement can be observed between experimental and calculated product concentrations. An Arrhenius type dependence of temperature on reaction rate has been found:

$k_{i}=k_{i, o} \exp \left(\frac{E_{a c t}}{R T}\right)$ 
where $\mathrm{k}_{\mathrm{i}, \mathrm{o}}$ is the preexponential factor, $\mathrm{E}_{\mathrm{act}}$ the activation energy and $\mathrm{R}$ is the gas constant.

Table 3.7 lists the values of the activation energy calculated by fitting the kinetic reaction rate constants of Table 6 to the Arrhenius equation. In this work, the most dependent steps on temperature correspond to the steps 1, 2 and $5\left(E_{a 1}, E_{a 2}\right.$ and $\left.E_{a 5}\right)$ that correspond to TAG consumption and reaction of DAG and MAG. The other reaction steps are small or nearly no dependent on reaction temperature. Only a few activation energy data have been found in the literature for the different steps involved in the glycerolysis process and comparison with other literature data is difficult. Guo and Xu (Guo \& Xu 2006) reported a single activation energy of $33.20 \mathrm{~kJ} / \mathrm{mol}$ for MAG formation by glycerolysis of sunflower oil with Novozym 435 in tert-butanol in a temperature range of 298-328 K. This value is of the same order as the highest values listed in Table $\mathbf{3 . 7}$ for some of the glycerolysis steps. Table 3.7 also presents activation energy values reported by Voll et al. (Voll et al. 2011) when using an order sequential $\mathrm{Bi}-\mathrm{Bi}$ mechanism in the fitting procedure for the glycerolysis of olive oil by Novozym 435 in tert-butanol. As can be clearly observed, different order of activation energies are obtained for the different steps proposed, especially for the activation energy values in the hydrolysis of MAG (step 11) and the reverse esterification of the free fatty acid (step 12). Table $\mathbf{3 . 7}$ also presents the activation energy values obtained by Valerio et al. (Valério, Krüger, et al. 2009) in the glycerolysis of olive oil by Novozym 435 in a surfactant system. Comparison among solvent systems (tertbutanol in this case) and surfactant systems is difficult since kinetic parameters for the description of enzyme behavior depend strongly on the solvent medium (Guo \& Xu 2006). In any case, TAG consumption by reaction with glycerol seems to be temperature dependent in all cases presented in Table 3.7. Valerio et al. (Valério, Krüger, et al. 2009) found that the most sensitive step to temperature was the reaction of TAG with MAG $\left(E_{a 5}\right)$ presenting an extremely high activation energy value.

Table 3.8 shows the root mean square deviation, rmsd, calculated from equation 14 for all the kinetic experiments performed in this work at different temperatures and mole ratio. The low rmsd values obtained for all the products composition, always less than $5 \mathrm{wt} \%$, proves that the model proposed fits the studied glycerolysis system at the experimental conditions essayed. Table $\mathbf{3 . 8}$ summarized the rmsd obtained by Voll et al. (Voll et al. 2011) when correlating kinetic data to an ordered-sequential $\mathrm{Bi} \mathrm{Bi}$ mechanism and the 
values obtained in this work. Similar values of rmsd are obtained for both model approaches.

Table 3.6. Calculated kinetic model parameters at different reaction temperatures ( $M R=$ 3:1, 10 wt\% Lipozyme 435 based on substrate weight). Objective function and root mean square deviation (wt \%) for glycerolysis products.

\begin{tabular}{|c|c|c|c|c|}
\hline \multirow{2}{*}{$\begin{array}{l}\text { Model } \\
\text { parameter }\end{array}$} & \multicolumn{4}{|c|}{ Reaction Temperature, $\mathrm{K}$} \\
\hline & 303 & 313 & 323 & 333 \\
\hline $\mathrm{k}_{1}$ & 0.0104 & 0.0164 & 0.0264 & 0.0357 \\
\hline $\mathrm{k}_{2}$ & 0.0106 & 0.0201 & 0.0248 & 0.0494 \\
\hline$k_{3}$ & 0.9132 & 0.9132 & 0.9167 & 0.9194 \\
\hline $\mathrm{k}_{4}$ & 0.0380 & 0.0395 & 0.0400 & 0.0436 \\
\hline$k_{5}$ & 0.0072 & 0.0114 & 0.0096 & 0.0174 \\
\hline $\mathrm{k}_{6}$ & -- & -- & -- & -- \\
\hline $\mathrm{k}_{7}$ & -- & -- & -- & -- \\
\hline $\mathrm{k}_{8}$ & 0.0041 & 0.0049 & 0.0046 & 0.0049 \\
\hline$k_{9}$ & 1.9020 & 1.9017 & 1.9017 & 1.9021 \\
\hline $\mathrm{k}_{10}$ & 0.0207 & 0.0223 & 0.0234 & 0.0241 \\
\hline $\mathrm{k}_{11}$ & 0.8967 & 0.8923 & 0.8911 & 0.8970 \\
\hline $\mathrm{k}_{12}$ & 1.8017 & 1.8041 & 1.8052 & 1.8069 \\
\hline O.F. & 0.0027 & 0.0021 & 0.0013 & 0.0030 \\
\hline \multicolumn{5}{|c|}{ Root mean squared deviation (wt \%) } \\
\hline TAG & 4.7 & 4.7 & 3.4 & 5.5 \\
\hline DAG & 0.6 & 0.7 & 0.9 & 0.6 \\
\hline MAG & 2.8 & 3.1 & 2.7 & 4.7 \\
\hline FFA & 0.4 & 0.6 & 0.7 & 0.7 \\
\hline Glycerol & 1.9 & 2.3 & 1.3 & 1.7 \\
\hline Water & 0.4 & 0.2 & 0.3 & 0.3 \\
\hline
\end{tabular}



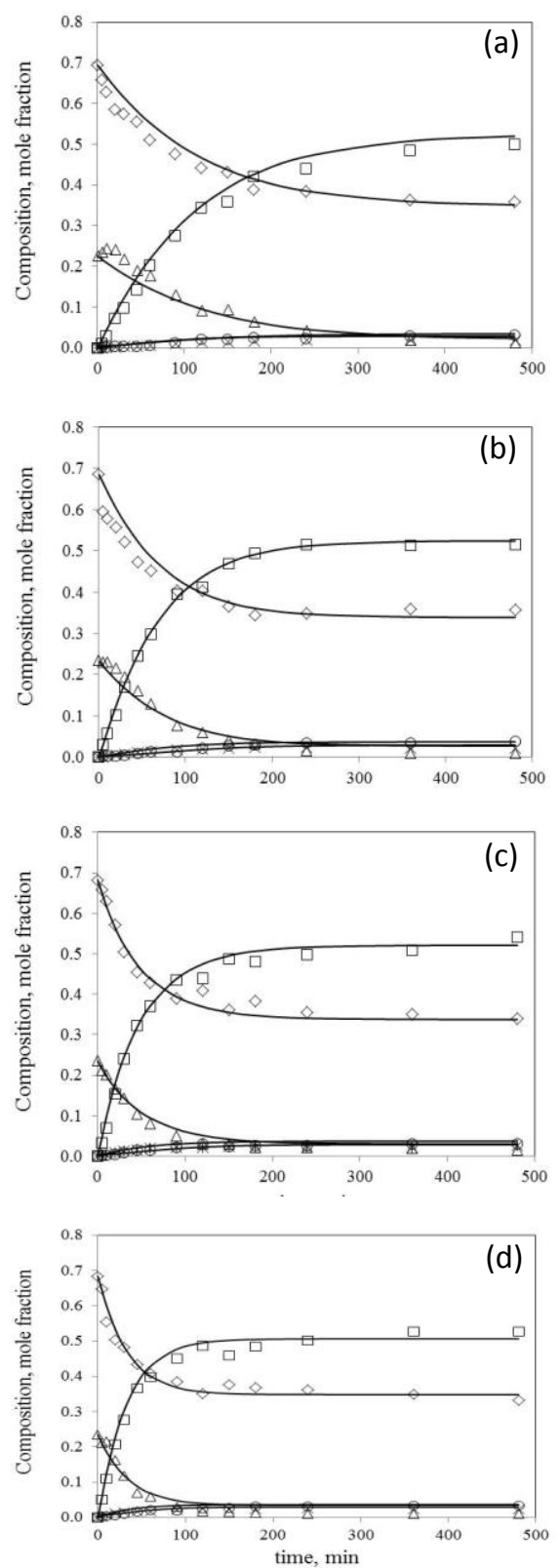

Figure 3.7. Time course for glycerolysis reaction at different temperatures: ( a) $303 \mathrm{~K}$, (b) $313 \mathrm{~K}$, (c) $323 \mathrm{~K}$, (d) $333 \mathrm{~K}$; 10 wt \% Lipozyme435 loading, MR =3:1; $\square$ MAG o FFA $\triangle$ TAG $\diamond$ glycerol $\times$ DAG. Continuous lines correspond to the model proposed in this work. Standard uncertainty $\mathrm{u}$ (mole fraction) $=0.02$. 
Table 3.7. Activation energy, $\mathrm{kJ} / \mathrm{mol}$, for the different steps in some glycerolysis systems

\begin{tabular}{lccc} 
Step & Ea,i (this work) & $\begin{array}{c}\text { Ea,i (Voll et al. } \\
\mathbf{2 0 1 1})^{*}\end{array}$ & $\begin{array}{c}\text { Ea,i (Valério, Krüger, } \\
\text { et al. 2009)** }\end{array}$ \\
1 & 35.18 & 18.30 & 27.91 \\
2 & 40.12 & $5.36 \cdot 10^{-5}$ & 0.06 \\
3 & 0.20 & -- & 47.08 \\
4 & 3.52 & $1.097 \cdot 10^{-4}$ & $2.68 \cdot 10^{-12}$ \\
5 & 20.64 & $8.397 \cdot 10^{-4}$ & 208.17 \\
6 & -- & 0.35 & 11.46 \\
7 & -- & 15.55 & 62.83 \\
8 & 4.10 & -- & 13.33 \\
9 & 0.001 & 2.33 & $1.11 \cdot 10^{-13}$ \\
10 & 4.20 & -- & 69.46 \\
11 & 0.009 & 45.46 & 71.48 \\
12 & 0.08 & 15.77 & 81.77 \\
\hline
\end{tabular}

$\left({ }^{*}\right)$ Solvent $=$ tert-butanol

$\left.{ }^{* *}\right)$ Surfactant system

Table 3.8. Root mean square deviation (wt\%), for glycerolysis products obtained with model equation (1-6)

\begin{tabular}{lllllll} 
TAG & DAG & MAG & FFA & Glycerol & Water & Reference \\
4.74 & 0.63 & 4.02 & 0.60 & 1.65 & 0.25 & This work \\
4.19 & 2.73 & 3.58 & 1.04 & -- & -- & (Voll et al. \\
& & & & & & 2011) \\
\hline
\end{tabular}

(-) data not reported

\section{Conclusions}

Glycerolysis of sardine oil using Lipozyme 435 has been carried out at different catalyst concentration, glycerol:oil mole ratio and reaction temperature. A homogeneous phase has been created by adding an optimized amount of tert-butanol based on phase equilibrium calculations. It has been proved that the external and internal diffusion can be 
considered negligible being the surface rate the controlling step. A lipase loading of 10 wt\% of unsieved Lipozyme 435 based on reactants weight has been used in all kinetic experiments. A mole ratio of glycerol:oil to $3: 1$ have been considered the optimum to produce more than 84 wt\% of MAG at $50^{\circ} \mathrm{C}$. Experimental kinetic data have been successfully correlated to a kinetic model based on the reversible elementary reactions. TAG consumption by reaction with glycerol and the reverse reaction are the steps more dependent on temperature. In the literature this results agrees for the TAG consumption; however different significance of kinetic parameters for most of the different steps involved in the glycerolysis system can be found in the literature and further studies are needed to be sure of the effect of the kinetic variables on the different steps. 


\section{CHAPTER 4}

\section{Production and concentration of}

monoacylglycerols rich in omega-3 polyunsaturated fatty acids by enzymatic glycerolysis and molecular distillation

Á. García Solaesa, M.T. Sanz, M. Falkeborg, S. Beltrán and Z. Guo (2016).

"Production and concentration of monoacylglycerols rich in omega-3 polyunsaturated fatty acids by enzymatic glycerolysis and molecular distillation"

Food Chemistry, 190, 960-967 



\section{Capítulo 4}

\section{Producción y concentración de monoglicéridos ricos en ácidos grasos poliinsaturados omega-3 por glicerolisis enzimática y destilación molecular}

\section{Resumen}

Como en capítulos anteriores, en este capítulo se ha estudiado la producción de monoglicéridos (MAG) ricos en ácidos grasos poliinsaturados omega-3 (AGPI n-3) por glicerolisis enzimática de aceite de sardina, con Lipozyme 435 y tert-pentanol, pero a mayor escala. Además la mezcla de reacción obtenida (con un 67\% de MAG) fue fraccionada por destilación molecular. El proceso de destilación se desarrolló por etapas en un equipo UIC KDL 5 (presión de vacío $10^{-3}$ mbar y flujo de alimentación $1.0 \mathrm{~mL} / \mathrm{min}$ ). La primera destilación se llevó a cabo a una temperatura de evaporación $\left(T_{E}\right)$ de $110{ }^{\circ} \mathrm{C}$, en la que se eliminó totalmente el glicerol y la mayoría de los ácidos grasos libres. La segunda destilación se optimizó a $155^{\circ} \mathrm{C}$, dando lugar a una corriente de destilado con un $91 \%$ deMAGy una recuperación global del 94\%. También se ha demostrado que la destilación molecular es capaz de concentrar los AGPI n-3 en forma de MAG en una tercera destilación a $125{ }^{\circ} \mathrm{C}$, quedando concentrados los MAG ricos en AGPI n-3 en la corriente más pesada. Por lo tanto, en este capítulo se desarrolla un proceso completo para la producción escalable de concentrados de AGPI n-3 en forma de MAG que pueden ser utilizados como emulsionantes e ingredientes alimentarios. 



\section{Abstract}

Production of monoacylglycerols (MAGs) rich in $\omega-3$ polyunsaturated fatty acids ( $n-3$ PUFAs) was conducted through short path distillation (SPD) of an acylglyceride mixture (containing 67\% MAGs) produced by enzymatic glycerolysis of sardine oil with glycerol. A stepwise SPD process in a UIC KDL 5 system (vacuum $10^{-3}$ mbar, feeding flow $1.0 \mathrm{~mL} / \mathrm{min}$ ) was proceeded: the first distillation performed at evaporator temperature $\left(T_{E}\right)$ of $110^{\circ} \mathrm{C}$ to remove glycerol completely and most of FFAs; and the second distillation at optimized $T_{E}$ $155^{\circ} \mathrm{C}$; resulting in a stream distillate with $91 \%$ purity and $94 \%$ overall recovery of MAGs. This work also demonstrated that SPD is able to concentrate n-3 PUFAs in MAG form by distilling at proper $T_{E}$ e.g. $125^{\circ} \mathrm{C}$, where n-3 PUFAs are concentrated in the residues. Moreover, this work mapped out a complete processing diagram for scalable production of n-3 PUFAs enriched MAGs as potential food emulsifier and ingredient.

\section{Introduction}

Fish oil is rich in $\omega-3$ (n-3) polyunsaturated fatty acids (PUFAs), such as eicosapentaenoic acid (EPA) and docosahexaenoic acid (DHA). The n-3 PUFAs play important roles in human health, and epidemiological and clinical studies have shown that EPA and DHA reduce the risk of coronary heart disease and help in brain, neural, and retinal developments (De Deckere et al. 1998; Komprda 2012; Kris-Etherton et al. 2002; Nichols et al. 2014).

There are several reasons for producing concentrates of n-3 PUFAs, but their chemical form has to be taken into account, as n-3 PUFAs in free fatty acids (FFAs), ethyl esters (EE), acylglycerols (AG), or phospholipids have different bioavailabilities. Studies have shown that human absorption of $n-3$ PUFAs in EE form is poor, primarily because EEs are poor substrates for pancreatic lipase. Although PUFAs in FFA form are absorbed more efficiently than PUFAs in EE or TAG form, they could present irritant effects and are very prone to auto-oxidation (Hernandez 2014; Lawson \& Hughes 1988b). Some studies have reported that the oxidative stability of PUFAs in TAG form is higher than PUFAs in EE form. Taking these considerations into account, MAG and DAG containing n-3 PUFAs are expected to have good application potentials, e.g. in functional foods, dietary 
supplements, or pharmaceuticals. Furthermore, these molecules, especially MAG, have excellent emulsifying properties, and MAG represent about $70 \%$ of the synthetic emulsifiers currently produced. For some applications, at least 90\% pure MAG is required (Fregolente et al. 2006). MAGs are usually produced by chemical glycerolysis employing inorganic alkaline catalysts at high temperatures. These processes are not suitable for heat-sensitive oils such as fish oils. Therefore, the enzymatic process has become an attractive alternative approach for polyunsaturated MAG production because the reaction can be carried out under mild conditions (Feltes et al. 2013).

The most widely used immobilized lipases used in enzymatic glycerolysis reactions are from Candida Antarctica (lipase B, Novozym 435), Rhizomucor miehei (Lipozyme RM IM), Pseudomonas fluorescens (IM-AK), and Thermomyces lanuginosus (Lipozyme TL IM) (Feltes et al. 2013). Several researchers use Novozym ${ }^{\circledR} 435$ as biocatalyst to mediate glycerolysis reactions due to its high MAG yield (Damstrup, Jensen, et al. 2006; Voll et al. 2011; Yang et al. 2005a). This lipase is not water-dependent, and works successfully in glycerolysis systems, even in the presence of solvents (Feltes et al. 2013). This is important, as solvents are usually required in glycerolysis reactions due to the immiscibility of the reactants (glycerol and oil or free fatty acids). Damstrup et al. (Damstrup et al. 2005) evaluated various solvents and concluded that the highest MAG yield by glycerolysis was obtained with tertiary alcohols, tert-butanol (TB) and tert-pentanol (TP). The relatively low log P value of these tertiary alcohols ( 0.35 and 0.89 , respectively) indicates both hydrophilic and hydrophobic characteristics, ensuring miscibility of the reactants. Moreover, Candida antarctica lipase B does not act on tertiary alcohols due to steric hindrance (Yang et al. 2005a). The liquid-liquid equilibria (LLE) of sardine oil + glycerol + tert-alcohols have been determined previously (Solaesa et al. 2013). LLE data help to minimize the amount of solvent needed to create a homogeneous reaction system, which leads to an improvement in mass transfer, obtaining high reactions yields in short reaction time.

For commercial interests, several methods have been reported for concentrating n-3 PUFA derivatives. Generally, a combination of techniques is used, such as an enzymatic reaction (hydrolysis, ethanolysis or glycerolysis) followed by molecular distillation, also known as short path distillation (SPD), low-temperature crystallization, urea complexation or 
supercritical fluid fractionation (Gámez-Meza et al. 2003; Lin et al. 2006; Xu 2003). Crystallization at low temperature has some technical limitations, such as molecular association and mixed-crystal formation and use of large quantities of organic solvents (Brown \& Kolb 1955). On the other hand, urea complexation presents some technical advantages in fractionation of PUFAs such as high efficiency and better selectivity. However, for concentrating PUFAs for human consumption the use of urea should be avoided, where formation of ethyl carbamate, an animal carcinogen is reported (Canas \& Yurawecz 1999). In case of supercritical fluid fractionation, most studies are directed towards fractionating the FFAs or their esters, which are more soluble in supercritical fluids. To design an efficient process, more knowledge on the solubility and phase equilibria of other compounds in the supercritical fluid is still needed (Van Dijk et al. 2012). Whereas, in SPD, compounds with different boiling points are separated under vacuum, which decreases the evaporation temperature and minimize the residence time, which enable heat-sensitive compounds to be separated with minimal thermal degradation. Therefore, this technology has been widely used in lipid areas. Most of the studies about the separation of MAG from DAG and TAG mixtures by SPD are focused on purification of saturated and monounsaturated compounds such as monoolein by glycerolysis from coconut oil (Zha et al. 2014), or high oleic sunflower oil (Zhu et al. 2011). Concentration of MAG and DAG by SPD from glycerolysis of palm olein (Yeoh et al. 2014), soybean oil (Fregolente et al. 2010) or camellia oil (Zheng et al. 2014) have been also reported. Processing of marine oils by SPD to remove pollutants and oxidation products has been reported (Oliveira \& Miller 2014; Oterhals \& Berntssen 2010), however, the studies focused on PUFA purification mainly obtained the PUFA concentrate in FFA or EE form (Breivik et al. 1997; Kahveci \& Xu 2011; Rossi et al. 2012; Zhang et al. 2013). Other studies have used SPD to remove ethyl esters subsequently to ethanolysis in order to obtain an acylglycerol product (mainly DAG) rich in DHA and EPA (Lyberg \& Adlercreutz 2008; Martín Valverde et al. 2012; Martín Valverde et al. 2013). However, the preparation of a product with a high concentration of MAG rich in n-3 PUFA has not been well documented yet.

In this work, acylglycerols rich in n-3 PUFA were prepared by enzymatic glycerolysis of sardine oil using immobilized Candida antarctica Lipase B (Lipozyme 435) in TP. The MAG 
fraction was purified using SPD. Different evaporation temperatures were evaluated to maximize the MAG recovery at high purity in the distillate. The main purpose of this study was to develop an effective distillation process for concentration of $n-3$ PUFA in MAG form.

\section{Experimental section}

\subsection{Materials}

Refined sardine oil was kindly provided by Industrias Afines S.L. (Spain). The fatty acid (FA) composition of sardine oil and the mole percentage of FAs at the sn-2 position of TAG is presented in Table 4.1 (Solaesa et al. 2014). More than $80 \%$ of DHA was found at sn-2 position.

Table 4.1. Fatty acid composition of sardine oil and at the sn- $2^{\mathrm{a}}$ position of TAG (\% mol) (Solaesa et al. 2014).

\begin{tabular}{lcc|c|cc}
\multirow{2}{*}{ Fatty acid } & \multicolumn{2}{c}{ Sardine oil } & \multicolumn{3}{c}{ Reaction compounds $^{b}$} \\
& TAG & $s n-2^{\mathrm{a}}$ & TAG & DAG & MAG \\
\hline $14: 0$ & $12.4 \pm 0.4$ & $41.8 \pm 0.1$ & $12 \pm 0.3$ & $12.5 \pm 0.4$ & $12.4 \pm 0.3$ \\
$16: 0$ & $22.8 \pm 0.2$ & $41.9 \pm 0.2$ & $29.4 \pm 0.7$ & $23.2 \pm 0.8$ & $23.0 \pm 0.6$ \\
$16: 1 \mathrm{n}-7$ & $12.5 \pm 0.1$ & $38.1 \pm 0.1$ & $11.9 \pm 0.3$ & $11.4 \pm 0.4$ & $13.1 \pm 0.3$ \\
$18: 0$ & $3.6 \pm 0.1$ & $6.7 \pm 0.1$ & $5.2 \pm 0.4$ & $3.2 \pm 0.2$ & $2.9 \pm 0.3$ \\
$18: 1 \mathrm{n}-9$ & $9.8 \pm 0.1$ & $16.6 \pm 0.1$ & $12.3 \pm 0.2$ & $8.3 \pm 0.5$ & $10.7 \pm 0.4$ \\
$18: 1 \mathrm{n}-7$ & $3.7 \pm 0.1$ & $10.1 \pm 0.1$ & $4.6 \pm 0.2$ & $2.8 \pm 0.2$ & $4.0 \pm 0.2$ \\
$18: 2 \mathrm{n}-6$ & $2.5 \pm 0.1$ & $32.7 \pm 0.1$ & $2.1 \pm 0.1$ & $2.6 \pm 0.1$ & $2.1 \pm 0.1$ \\
$18: 3 \mathrm{n}-3$ & $1.0 \pm 0.1$ & $29.0 \pm 0.1$ & $0.8 \pm 0.1$ & $1.1 \pm 0.1$ & $0.9 \pm 0.1$ \\
$18: 4 \mathrm{n}-3$ & $3.3 \pm 0.1$ & $26.4 \pm 0.1$ & $2.0 \pm 0.1$ & $3.2 \pm 0.2$ & $2.9 \pm 0.1$ \\
$20: 3 \mathrm{n}-3$ & $1.3 \pm 0.3$ & $19.6 \pm 0.1$ & $0.9 \pm 0.1$ & $1.0 \pm 0.1$ & $1.2 \pm 0.1$ \\
$20: 5 \mathrm{n}-3$ & $18.3 \pm 0.3$ & $12.1 \pm 0.1$ & $11.9 \pm 0.6$ & $19.4 \pm 0.5$ & $18.7 \pm 0.6$ \\
$22: 5 \mathrm{n}-3$ & $1.8 \pm 0.1$ & $76.4 \pm 0.1$ & $1.4 \pm 0.1$ & $1.9 \pm 0.1$ & $1.7 \pm 0.1$ \\
$22: 6 \mathrm{n}-3$ & $7.0 \pm 0.2$ & $82.8 \pm 0.1$ & $5.5 \pm 0.4$ & $9.4 \pm 0.6$ & $6.4 \pm 0.3$ \\
\hline
\end{tabular}

(a) $\% s n-2=[\mathrm{mol} \% s n-2$ fatty acid / (mol \% fatty acid in TAG.3) $] \cdot 100$

(b) Obtained at optimum conditions 


\subsection{Enzymatic glycerolysis of sardine oil}

Different vials containing one gram of mixture of sardine oil, glycerol and TP were incubated at $50^{\circ} \mathrm{C}$ in a water bath with orbital agitation. Different molar ratios of substrates and enzyme dosages were studied. The amount of TP added was calculated on the basis of previous studies on LLE (Solaesa et al. 2013). It was fixed at mass ratio of 1.5:1 (TP:substrates). At selected time intervals (from ten minutes up to eight hours), the reaction mixture was withdrawn and filtered through a microfilter $(0.45 \mu \mathrm{m}$, Sartorius $\mathrm{RC})$ to stop the reaction by removing the lipase. All samples were stored at $-18^{\circ} \mathrm{C}$ prior to analysis. Samples were analyzed by HPLC to quantify the lipid compounds (TAG, DAG, MAG and FFA).

\subsection{Fractionation of lipid classes by HPLC}

The lipid compounds (MAG, DAG, TAG and FFA) were quantified by normal-phase high performance liquid chromatography (NP-HPLC). The chromatographic apparatus consisted of a HPLC system (Agilent Technologies 1200 Series Model, Santa Clara, CA, USA) formed by a quaternary pump and an auto-injector. The chromatographic separation of the compounds was carried out at room temperature with a Lichrospher Diol column $(5 \mu \mathrm{m}, 4$ $\mathrm{mm} \times 250 \mathrm{~mm}$ ) and detection was performed in an evaporative light scattering detector (ELSD) at $35^{\circ} \mathrm{C}$ and $0.35 \mathrm{MPa}$. Gradient elution was achieved by mobile phases A (isooctane) and B (methyl tert-butyl ether/acetic acid, 99.9:0.1, v/v). The course of the gradient was as follows: first, solvent A was flowing for $1 \mathrm{~min}$, after that, solvent B was added in three steps, up to $10 \%$ in $10 \mathrm{~min}$, to $44 \%$ in $22 \mathrm{~min}$ and to $100 \%$ in $30 \mathrm{~min}$. Subsequently, solvent B was decreasing down to $0 \%$ in 17 min to come back to the beginning conditions. Finally, the stationary phase was rinsed with solvent $A$ for 2 min. Injection volumes of $10 \mu \mathrm{L}$ and the elution flow-rate of $1 \mathrm{~mL} / \mathrm{min}$ were used in all experiments. Individual compounds were identified and quantified using a calibration curve of the corresponding standard compound of DAG (mixture of dipalmitin and diolein), MAG (mixture of monopalmitin, 1-monoolein, 2-monoolein and monodocosahexaenoin) and FFA (oleic and palmitic acids) in tert-pentanol. In case of TAG, the calibration curve used was made with the refined sardine oil (TAG $\geq 99 \%$ ) because the 
response factor of the oil as a complex mixture of TAG was very different than the pure standards of TAG. The fractions detected by NP-HPLC under optimal reaction conditions were collected according to their retention times. Separations were repeated at least six times in order to obtain enough sample to evaluate the FA profile of each compound (MAG, DAG and TAG).

\subsection{Scaling up lipase-catalyzed glycerolysis}

Eight hundred grams of reaction mixture with a reactant mole ratio 3:1 (glycerol/oil), with a TP/substrates weight ratio of $1.5: 1$ and 5 wt\% of Lipozyme 435 were placed in a $1 \mathrm{~L}$ jacketed batch reactor and heated to $50{ }^{\circ} \mathrm{C}$ under $200 \mathrm{rpm}$ stirring. The glycerolysis reaction took place for $2 \mathrm{~h}$. After that, the enzyme particles were removed by double filtration. The first one was through the filter sited at the bottom of the reactor and the second filtration was under vacuum. The whole procedure was carried out four times to produce enough reaction mixture to perform the SPD experiments. Before SPD, the solvent was evaporated under vacuum using a rotary evaporator (Vacuubrand PC 3001 VARIOpro) at $55{ }^{\circ} \mathrm{C}$. It could be reused by using molecular sieves to eliminate the water content.

\subsection{Purification of MAG by SPD}

The enzymatic glycerolysis reaction products were separated using SPD (KDL 5, UIC GmbH, Alzenau-Hoerstein, Germany) at Aarhus University. To obtain a highly pure MAG fraction, two-step distillations were performed varying the evaporation temperature from $110{ }^{\circ} \mathrm{C}$ to $195^{\circ} \mathrm{C}$. The first step was optimized at $110^{\circ} \mathrm{C}$ to remove glycerol and most of FFA. The residue obtained in the first distillation at $110{ }^{\circ} \mathrm{C}$ was the feeding material in the second distillation. The evaporator temperatures were varied in the second distillation from 135 to $195{ }^{\circ} \mathrm{C}$ to distillate the MAG fraction with the minimum of DAG possible. The other operation variables were fixed as follows: $375 \mathrm{rpm}$ as rotation speed, $1 \mathrm{~mL} / \mathrm{min}$ as feed rate, $10^{-3} \mathrm{mbar}$ of vacuum, $45^{\circ} \mathrm{C}$ as temperature of feed reservoir, and $50{ }^{\circ} \mathrm{C}$ as condenser temperature. 
Moreover, once MAG was purified, a third distillation was carried out to distillate MC-SFA (medium chain-saturated fatty acid) MAG, leaving a concentrate fraction of LC-PUFA MAG in the residue.

The lipid profiles of the residue and distillate fractions were analyzed by thin layer chromatography coupled with a flame ionization detector (TLC-FID). The fractions from the third distillation and some fractions from the second one were also analyzed by GCFID to determine their FA composition.

\subsection{Acylglycerol composition analysis by TLC-FID}

Samples from residue and distillate fractions obtained from SPD were analyzed by TLC-FID (latroscan MK-6 s, Bechenheim, Germany) at Aarhus University to quantify the reaction compounds (TAG, DAG, MAG, FFA and glycerol). The samples were diluted to $20 \mathrm{mg} / \mathrm{mL}$ in chloroform/methanol $(3: 1, v / v)$ and $1 \mu \mathrm{L}$ was spotted onto silica-coated quartz rods. Samples were developed with the developing system of hexane/diethyl ether/acetic acid $(40: 30: 1, \mathrm{v} / \mathrm{v} / \mathrm{v})$. The rods were dried for $5 \mathrm{~min}$ at $120{ }^{\circ} \mathrm{C}$ prior to analysis. The area percentages of lipid classes were used for the calculation of product yields.

\subsection{Fatty acid composition by GC-FID}

The samples were transferred to screw-capped tubes to carry out the conversion to methyl esters by the AOAC method(AOAC Official Method 991.39 2000)and subsequent analysis by gas chromatography (GC) with an Agilent gas chromatograph (6890N Network GC System, Santa Clara CA, United States) equipped with a flame ionization detector (FID). A fused silica capillary column (OmegawaxTM-320, $30 \mathrm{~m} \times 0.32 \mathrm{~mm}$ i.d.) was used. The separation was carried out with helium $(1.8 \mathrm{~mL} / \mathrm{min})$ as carrier gas. The column temperature was programmed starting at a constant value of $180{ }^{\circ} \mathrm{C}$ during $20 \mathrm{~min}$, heated to $200{ }^{\circ} \mathrm{C}$ at $1{ }^{\circ} \mathrm{C} / \mathrm{min}$, held at $200{ }^{\circ} \mathrm{C}$ during $1 \mathrm{~min}$, heated again to $220^{\circ} \mathrm{C}$ at $5{ }^{\circ} \mathrm{C} / \mathrm{min}$ and finally held at $220^{\circ} \mathrm{C}$ for $20 \mathrm{~min}$. A split injector $(50: 1)$ at $250^{\circ} \mathrm{C}$ was used. The FID was also heated at $250{ }^{\circ} \mathrm{C}$. The injection volume was $1 \mu \mathrm{L}$. Most of the FAMEs were identified by comparison of their retention times with those of chromatographic standards (Sigma Chemical Co.).The method and the calibration procedure was developed 
previously and the compounds were quantified related to the area of an internal standard (methyl tricosanoate) as indicated by the AOAC method (AOAC Official Method 991.39 2000).

\section{Results and discussion}

\subsection{Effect of substrate molar ratio and enzyme concentration in the production of MAG}

To determine an optimum substrate mole ratio, the effect of glycerol/oil mole ratio on the reaction rate was studied at three levels $(1: 1,3: 1$ and $5: 1)$ at $50{ }^{\circ} \mathrm{C}$ with 10 wt\% of lipase. The results are shown in Fig. 4.1A. The substrate mole ratio can influence the reaction in different ways. An increase in glycerol amount will increase the theoretical equilibrium conversion, and shift the equilibrium towards MAG production. On the other hand, an excess of glycerol will affect the polarity as well as the stability of the system (Yang et al. 2005a). The MAG yield at a mole ratio of $1: 1$ was around $60 \%$, whereas values up to $90 \%$ were reached at a mole ratio of 5:1. There was little difference in MAG yield between the 3:1 and 5:1 glycerol/oil mole ratios, reaching in both cases around $90 \mathrm{wt} \%$ of MAG yield. Based on these results, a glycerol/oil molar ratio of 3:1 was selected as optimum in the present study. This result agrees with previous studies on glycerolysis of different types of oils. Zhu et al. (Zhu et al. 2011) chose a substrate molar ratio of 3:1 using high oleic sunflower oil in a mixture of TP and TB medium. Zhong et al. (Zhong et al. 2009) selected 3.5:1 glycerol/soybean oil molar ratio in their study on glycerolysis reaction in a mixture of TB/isopropanol (80:20, v/v). Pawongrat et al. (Pawongrat et al. 2007) used methyl tertbutyl ether as solvent and showed that the optimum mole ratio of glycerol to tuna oil for MAG production was 3:1

In the enzyme load study, glycerolysis of sardine oil was carried out using Lipozyme ${ }^{\circledR} 435$ at a load of 5 and 10 wt\% based on reactants weight, with a glycerol/oil mole ratio of 3:1. The results are shown in Fig. 4.1B. As expected, the MAG yield increased with increasing lipase concentration, which may be due to higher number of active sites. MAG production was around 90 and $80 \%$ (expressed free of glycerol and TP) at 10 and 5\% of lipase loading, 
respectively. When using $10 \%$ lipase, around $80 \%$ of MAG was reached in less than 30 minutes. To achieve the same yield of MAG with $5 \%$ lipase, 4 hours was needed. The best enzyme load should be evaluated from an economical point of view, evaluating the effect of longer reaction time and higher enzyme loading. In this work, 5\% Lipozyme 435 and 2 hours of reaction was chosen to scale up the process with $67 \%$ of MAG.

\subsection{Scale-up reaction under optimal conditions for MAG} production

According to previous results (section 3.1), a high yield of MAG (67 wt\%) can be reached in 2 hours of reaction time, with a reactants molar ratio 3:1 (glycerol/sardine oil) and a lipase load of $5 \%$ based on reactants weight. Large-scale reaction was performed at these conditions in a $1 \mathrm{~L}$ batch reactor at $50{ }^{\circ} \mathrm{C}$ under $200 \mathrm{rpm}$ stirring. The final MAG content obtained by glycerolysis of $1 \mathrm{~g}$ and $800 \mathrm{~g}$ of reaction mixture were of the same order (67 and $70 w t \%$, respectively), hence, scaling up did not affect the yield of the enzymatic process. The slight increase in MAG yield at large scale could be due to the use of mechanic propeller agitation instead of orbital agitation in vials. The lipase and solvent were removed by filtration and evaporation, respectively, before SPD. The lipase and recovered TP could be reused for further reactions.

The FA composition of acylglycerols produced at optimal conditions can be seen in Table 4.1. These results revealed that the TAG fraction contained more palmitic, stearic and oleic acids, compared to the DAG and MAG fractions. On the other hand, EPA and DHA were higher in the DAG and MAG fractions. The produced MAG had a similar FA profile to the starting sardine oil, and the sum of EPA, DPA (docosapentaenoic acid) and DHA was almost $27 \%$ as in the sardine oil.

\subsection{Purification of MAG by SPD}

The products from glycerolysis consist mainly of MAG and DAG, some glycerol, and a small amount of FFA and TAG. SPD was performed to obtain a highly pure MAG fraction after the glycerolysis reaction. The order of removalin the distillate stream would follow the 
same order as vapor pressure of different acylglycerols: Glycerol $<$ FFA $<$ MAG $<$ DAG $<$ TAG (Yeoh et al. 2014).
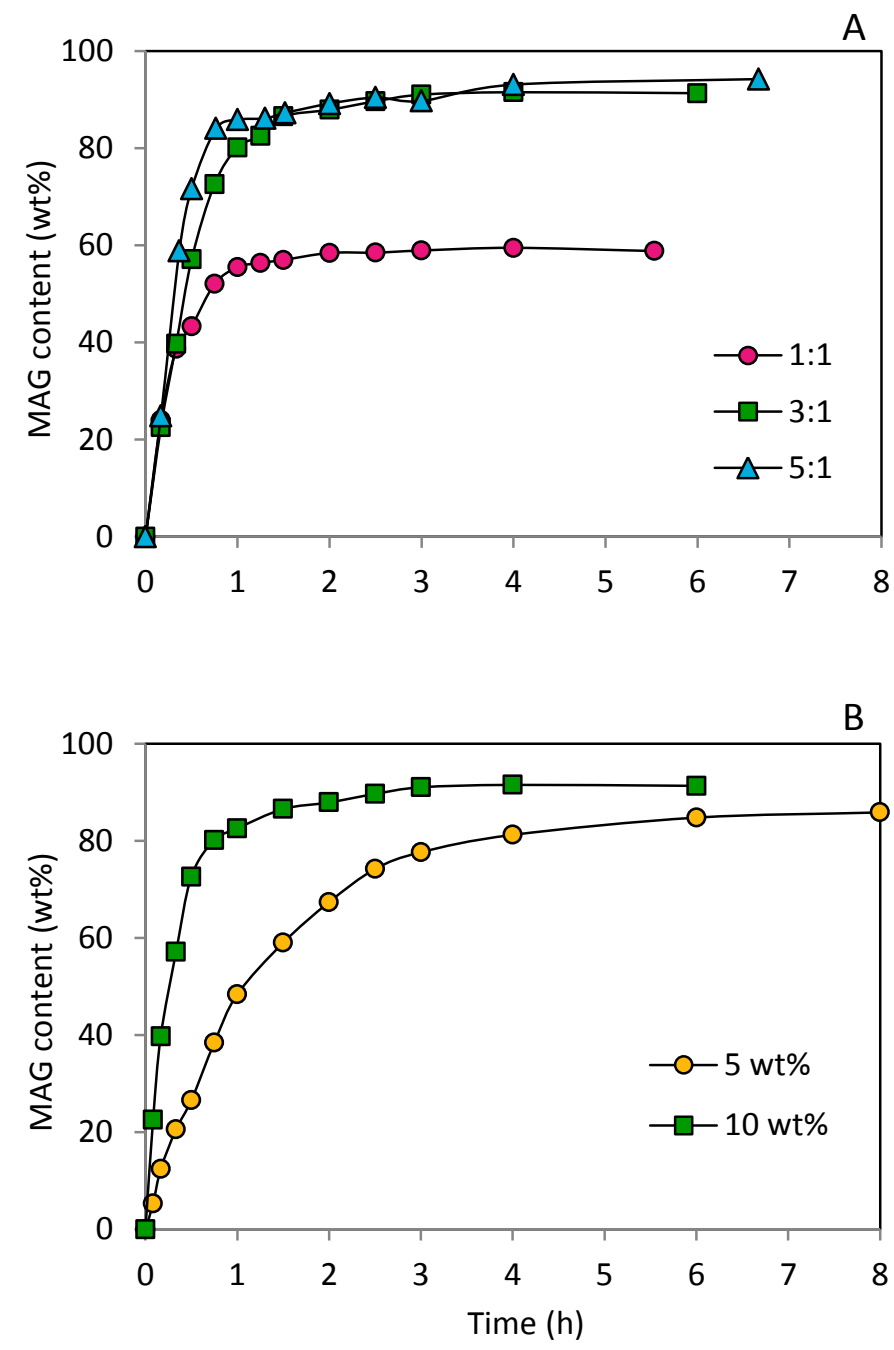

Fig. 4.1. Effect of kinetic parameters in MAG production by glycerolysis of sardine oil. (A) Effect of glycerol to oil molar ratio (1:1, 3:1, 5:1); reaction conditions: 10 wt\% of Lipozyme $435,50{ }^{\circ} \mathrm{C}$ and 1.5:1 (weight ratio. tert-pentanol:substrates). (B) Effect of lipase dosage (5 and $10 \mathrm{wt} \%$, based on total substrates); reaction conditions: 3:1 glycerol to sardine oil molar ratio, $50{ }^{\circ} \mathrm{C}$ and $1.5: 1$ (weight ratio, tert-pentanol:substrates). 
However, the chain length and the number of double bonds in the fatty acid also influence the distillation order. The rate of fatty acids evaporation follows the order of 14:0 > 16:0> 18:1 > EPA > DHA (Liang \& Hwang 2000; Rossi et al. 2012). Due to the variability in the FA profile of fish oils, it is expected that different acylglycerols could be distilled the same evaporator temperature; hence, MAG with the highest boiling points could be distillated together with DAG with the lowest boiling points.

Many parameters can influence the separation performance in SPD. Previous studies about SPD show that the operating parameters having greater effect on the separation efficiency are evaporator temperature, feed flow rate, and vacuum pressure (Fregolente et al. 2010; Yeoh et al. 2014). The feed temperature, the condenser temperature, and rotational speed have little influence on the separation (Zhang et al. 2013; Zhu et al. 2011), and in this work, these parameters were fixed at $45^{\circ} \mathrm{C}, 50^{\circ} \mathrm{C}$ and $375 \mathrm{rpm}$, respectively. For practical operation, the lowest vacuum should be used to maximize the separation efficiency (Xu et al. 2002). In this case, the vacuum system included a diffusion pump and a rotary vane pump, achieving $10^{-3}$ mbar. Feed flow rate is an important operating variable in SPD. High feed flow rate reduces the operating time but results in decreased separation and lower acylglycerol purity; while low flow rate fosters efficient separation, yetincreases the processing time (Yeoh et al. 2014). In several studies, the feeding rate was around $1.5 \mathrm{~mL} / \mathrm{min}$ for a UIC KDL1 system (Zha et al. 2014; Zhang et al. 2013) and for MD-S80 distiller (Zhu et al. 2011). In this work, the feed flow rate for a UIC KD5 system was set to $1 \mathrm{~mL} / \mathrm{min}$.

Some studies showed that the evaporator temperature is the key factor in the separation of the compounds (Fregolente et al. 2007; Zha et al. 2014). In this work, a two-step distillation was performed to study the effect of $T_{E}$ in the separation of acylglycerols. The first step was done at $110^{\circ} \mathrm{C}$ to remove the glycerol and the FFA. In the second distillation, the temperatures were varied in the range from $135^{\circ} \mathrm{C}$ to $195^{\circ} \mathrm{C}$. When feeding the glycerolysis product mixture to the SPD, it was divided into two streams. Part of the feed (F) was collected in the distillate stream (D) while the rest remained in the residue stream (R). The yield and the recovery are important indexes to evaluate the performance of SPD process. The yield of $R$ or $D$ is calculated by the following equation (Eq. 1): 
Yield $_{\mathrm{R} / \mathrm{D}}(\%)=\frac{M_{R / D}}{M_{\text {Feed }}} \times 100$

where $\mathrm{M}$ is the amount in grams of $\mathrm{R}$ or $\mathrm{D}$ collected. The recovery of a certain component was determined by using Eq. (2):

Recovery of a substance, $\mathrm{i}(\%)=\frac{M_{R / D} \times C_{i, R / D}}{M_{\text {Feed }} \times C_{i, \text { Feed }}} \times 100$

where $\mathrm{C}$ is the concentration as area \% measured by TLC-FID of each compound in the residue or distillate collected, and “i” represents TAG, DAG, MAG, FFA or glycerol. The acylglycerol compositions, yield, recovery and loss of the compounds in the $\mathrm{D}$ and $\mathrm{R}$ streams for different temperatures are shown in Table 4.2. The sum of $R$ and $D$ recoveries were sometimes above or below $100 \%$, due to weight losses, which are expected in the process (Kahveci \& Xu 2011), and analytical errors.

As shown in Table 4.2, the sample initially fed to SPD at the beginning contains $10.4 \%$ TAG, 17.4\% DAG, 64.6\% MAG, 0.6\% FFA, and 7.0\% glycerol. As a general trend, higher yields of distillate were obtained at higher $\mathrm{T}_{\mathrm{E}}$. Increasing $\mathrm{T}_{\mathrm{E}}$ leads to higher heat transfer rates, and therefore, more molecules could be evaporated (Fregolente et al. 2010). As the results shown in Table 4.2, the first step of SPD is a pre-distillation at $110^{\circ} \mathrm{C}$ in order to remove the glycerol and part of FFA (D1 in Fig. 4.3). The glycerol was completely distillated at this temperature. However, the small vapor pressure difference between some FFA and some MAG make a complete separation of both components difficult. Therefore, a complete removal of FFAs was not possible if MAG would be not distillated at all. $A T_{E}$ of $135^{\circ} \mathrm{C}$ would be necessary to remove all FFAs, as it can be seen in Table 4.2, no FFA were detected in the residue stream under this temperature. This fact coincides with the study of Zhu et al (Zhu et al. 2011), where they concluded that most of the glycerol and FFA could be separated from their glycerolysis reaction mixture when the evaporation temperature was set over $140{ }^{\circ} \mathrm{C}$, under vacuum of $1-5 \mathrm{~Pa}$ (operation pressure higher than in this work), a rotational speed of $200 \mathrm{rpm}$, and feed flow rate of $1.5 \mathrm{~mL} / \mathrm{min}$. However, at this temperature the loss of MAG in the distillate is important. In this work, the recovery of FFA in the distillate stream at $110{ }^{\circ} \mathrm{C}$ reached values up to $63.5 \%$. At this $T_{E}$, nearly all TAG and DAG were collected in the residue stream, while $8 \%$ of the MAG fraction was lost in 
the distillate stream. On the other hand, a good MAG recovery in the residue is obtained, almost $90 \%$.
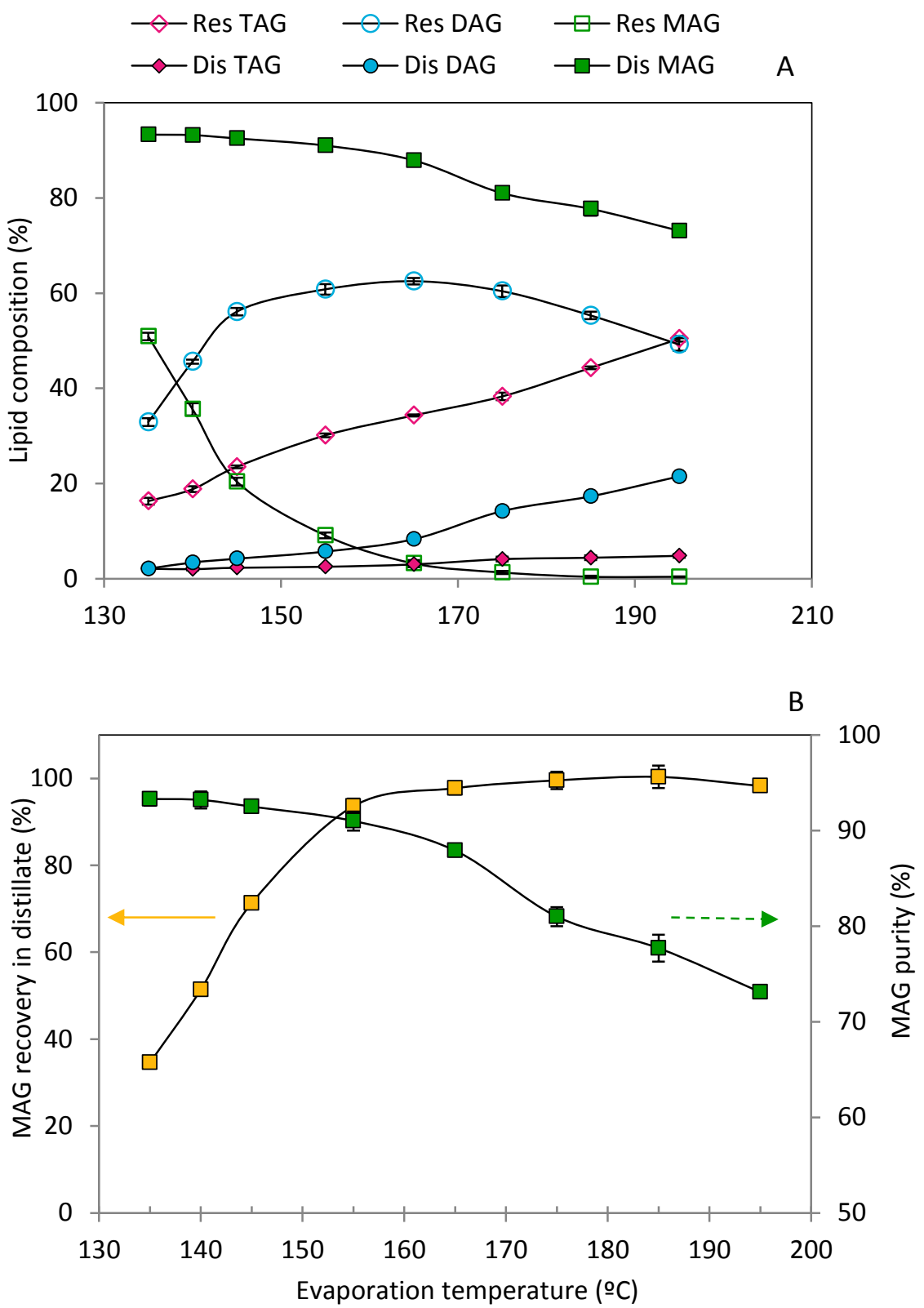

Fig. 4.2. (A) Acylglycerols composition in residue and distillate fractions at different evaporation temperatures. (B) MAG recovery and purity in distillate at different evaporation temperatures. 
Table 4.2. Acylglycerol compositions, yield, and recovery of all components obtained in two steps distillation at different evaporation temperatures.

\begin{tabular}{|c|c|c|c|c|c|c|c|c|c|c|c|c|}
\hline \multirow{2}{*}{$\begin{array}{l}\text { Evaporator } \\
\text { temperature } \\
\left({ }^{\circ} \mathrm{C}\right)\end{array}$} & \multirow[b]{2}{*}{ Streams } & \multirow[b]{2}{*}{$\begin{array}{l}\text { Yield } \\
(\%)\end{array}$} & \multicolumn{2}{|c|}{ TAG } & \multicolumn{2}{|c|}{ DAG } & \multicolumn{2}{|c|}{ MAG } & \multicolumn{2}{|c|}{ FFA } & \multicolumn{2}{|c|}{ Glycerol } \\
\hline & & & Area \% & $\begin{array}{c}\text { Recovery } \\
\%\end{array}$ & Area \% & $\begin{array}{c}\text { Recovery } \\
\%\end{array}$ & Area \% & $\begin{array}{c}\text { Recovery } \\
\%\end{array}$ & Area \% & $\begin{array}{c}\text { Recovery } \\
\%\end{array}$ & Area \% & $\begin{array}{c}\text { Recovery } \\
\%\end{array}$ \\
\hline $1^{\text {st }}$ distillation & Feed & - & $10.4 \pm 0.1$ & & $17.4 \pm 0.3$ & & $64.6 \pm 0.5$ & & $0.63 \pm 0.08$ & & $7.0 \pm 0.8$ & \\
\hline \multirow[t]{2}{*}{110} & Residue & 85.4 & $11.9 \pm 0.5$ & $97 \pm 5$ & $20.0 \pm 0.3$ & $98.4 \pm 3.2$ & $67.8 \pm 1.6$ & $89.6 \pm 2.8$ & $0.33 \pm 0.02$ & $44.7 \pm 8.4$ & - & - \\
\hline & Distillate & 14.6 & $4.5 \pm 0.3$ & $6.4 \pm 0.5$ & $7.0 \pm 0.5$ & $5.9 \pm 0.5$ & $36 \pm 1$ & $8.1 \pm 0.3$ & $2.74 \pm 0.03$ & $63.5 \pm 8.8$ & $49.8 \pm 0.5$ & $104 \pm 13$ \\
\hline $2^{\text {nd }}$ distillation & Feed & - & $10.6 \pm 0.5$ & & $25.6 \pm 0.9$ & & $63.3 \pm 0.7$ & & $0.52 \pm 0.04$ & & & \\
\hline \multirow[t]{2}{*}{135} & Residue & 76.5 & $16.3 \pm 0.7$ & $118 \pm 11$ & $32.9 \pm 0.8$ & $98.2 \pm 5.8$ & $50.9 \pm 0.8$ & $61.5 \pm 1.5$ & - & - & & \\
\hline & Distillate & 23.5 & $2.1 \pm 0.2$ & $4.6 \pm 0.7$ & $2.1 \pm 0.3$ & $1.9 \pm 0.3$ & $93.3 \pm 0.7$ & $34.6 \pm 0.5$ & $2.50 \pm 0.04$ & $114 \pm 11$ & & \\
\hline \multirow[t]{2}{*}{140} & Residue & 65.0 & $18.8 \pm 0.6$ & $116 \pm 9$ & $45.6 \pm 0.4$ & $116 \pm 5$ & $35.6 \pm 1.3$ & $36.5 \pm 1.6$ & - & - & & \\
\hline & Distillate & 35.0 & $2.0 \pm 0.2$ & $7 \pm 1$ & $3.4 \pm 0.2$ & $4.6 \pm 0.4$ & $93.2 \pm 0.9$ & $51.5 \pm 0.9$ & $1.51 \pm 0.02$ & $103 \pm 9$ & & \\
\hline \multirow[t]{2}{*}{145} & Residue & 51.2 & $23.5 \pm 0.3$ & $114 \pm 7$ & $56.1 \pm 0.8$ & $112 \pm 6$ & $20.4 \pm 0.8$ & $16.7 \pm 0.8$ & - & & & \\
\hline & Distillate & 48.8 & $2.3 \pm 0.1$ & $10 \pm 1$ & $4.2 \pm 0.4$ & $8 \pm 1$ & $92.5 \pm 0.4$ & $71.3 \pm 0.9$ & $1.06 \pm 0.05$ & $100 \pm 13$ & & \\
\hline \multirow[t]{2}{*}{155} & Residue & 34.9 & $30.1 \pm 0.4$ & $99 \pm 6$ & $60.8 \pm 1.1$ & $82.9 \pm 4.4$ & $9.1 \pm 0.6$ & $5 \pm 0.4$ & - & - & & \\
\hline & Distillate & 65.1 & $2.5 \pm 0.2$ & $15 \pm 2$ & $5.7 \pm 0.4$ & $14.5 \pm 1.5$ & $91 \pm 1$ & $93.6 \pm 1.8$ & $0.82 \pm 0.04$ & $107 \pm 13$ & & \\
\hline \multirow[t]{2}{*}{165} & Residue & 29.6 & $34.3 \pm 0.2$ & $95.8 \pm 5.1$ & $62.5 \pm 0.7$ & $72.3 \pm 3.4$ & $3.2 \pm 0.7$ & $1.5 \pm 0.3$ & - & - & & \\
\hline & Distillate & 70.4 & $3.0 \pm 0.6$ & $19.9 \pm 4.9$ & $8.3 \pm 0.7$ & $22.8 \pm 2.7$ & $87.9 \pm 0.6$ & $97.7 \pm 1.4$ & $0.78 \pm 0.04$ & $110 \pm 14$ & & \\
\hline \multirow[t]{2}{*}{175} & Residue & 22.2 & $38.3 \pm 0.8$ & $80.3 \pm 5.5$ & $60.4 \pm 1.2$ & $52.4 \pm 2.9$ & $1.3 \pm 0.3$ & $0.5 \pm 0.1$ & - & - & & \\
\hline & Distillate & 77.8 & $4.1 \pm 0.5$ & $30.1 \pm 5.1$ & $14.2 \pm 0.6$ & $43.1 \pm 3.3$ & $81 \pm 1$ & $99.5 \pm 2.0$ & $0.71 \pm 0.02$ & $111 \pm 12$ & & \\
\hline \multirow[t]{2}{*}{185} & Residue & 18.3 & $44.3 \pm 0.3$ & $76.3 \pm 4.1$ & $55.3 \pm 0.8$ & $39 \pm 2$ & $0.4 \pm 0.2$ & $0.1 \pm 0.1$ & - & - & & \\
\hline & Distillate & 81.7 & $4.4 \pm 0.5$ & $33.9 \pm 5.5$ & $17.3 \pm 0.3$ & $55.2 \pm 2.9$ & $77.7 \pm 1.4$ & $100.3 \pm 2.6$ & $0.62 \pm 0.03$ & $101 \pm 13$ & & \\
\hline \multirow[t]{2}{*}{195} & Residue & 14.9 & $50.4 \pm 0.6$ & $70.9 \pm 4.2$ & $49.2 \pm 1.3$ & $28.7 \pm 1.8$ & $0.4 \pm 0.1$ & $0.1 \pm 0.1$ & - & - & & \\
\hline & Distillate & 85.1 & $4.8 \pm 0.4$ & $39 \pm 5$ & $21.5 \pm 0.6$ & $71.5 \pm 4.5$ & $73.1 \pm 0.2$ & $98 \pm 1$ & $0.54 \pm 0.04$ & $92 \pm 14$ & & \\
\hline
\end{tabular}


Sardine oil + Glycerol + TP

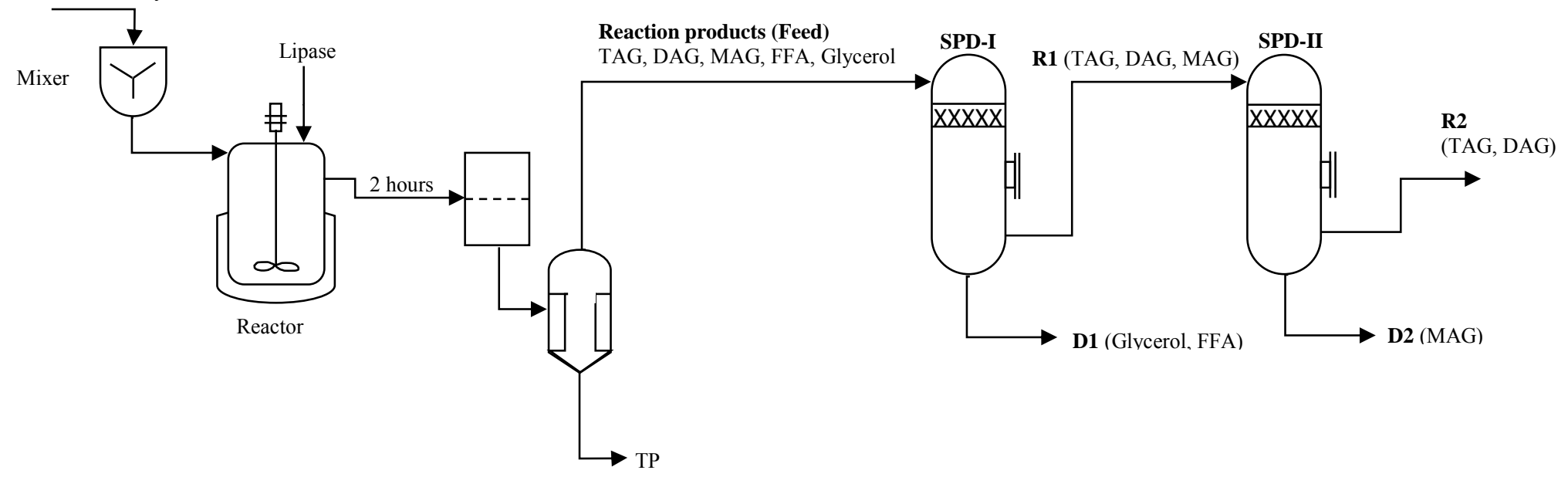

Fig. 4.3. Global scheme of the process to produce concentrated MAG fraction by glycerolysis reaction and two-step distillation at selected evaporation temperatures with the main components in each stream. 
The variation in the lipid profile of $D$ and $R$ streams are represented as function of evaporation temperatures in Fig. 4.2A. It can be observed that TAG and DAG start to evaporate at $155^{\circ} \mathrm{C}$, so at this temperature, the purity of MAG in the distillate decreased. However, TAG in D fraction was lower than $5 \%$ for all temperatures; while DAG, being more volatile than TAG, steadily increases from $155^{\circ} \mathrm{C}$, and consequently a maximum in DAG profile with temperature in the $\mathrm{R}$ stream is observed.

Fig. 4.2B shows the relation between MAG recovery in the distillate and MAG purity at different $T_{E}$. It can be seen that the MAG recovery increased until $155^{\circ} \mathrm{C}$, reaching more than 93\%. Up from there, it kept constant because MAG distillation was complete. On the other hand, the MAG purity starts to decrease at $T_{E}$ over $155^{\circ} \mathrm{C}$ since mainly DAG starts to be distillated. At this temperature, the composition of the D stream was $91.0 \%$ MAG, 5.7\% DAG, and $2.5 \%$ TAG while just $5 \%$ MAG was lost in the R stream. At $165^{\circ} \mathrm{C}$, the MAG loss in the R stream was only $1.5 \%$, but the recovery of DAG in the distillate was more than $20 \%$ decreasing MAG concentration in the D stream. With these results, it can be concluded that the optimal $T_{E}$ to distillate MAG fraction is $155^{\circ} \mathrm{C}$. At this $T_{E}$, it was possible to concentrate MAG to $91 \%$ from a mixture that initially contained $63 \%$ of MAG.

Several authors demonstrated that SPD is useful to concentrate n-3 PUFA in EE form or even as FFA (Breivik et al. 1997; Kahveci \& Xu 2011; Liang \& Hwang 2000; Zhang et al. 2013). In this work, GC analyses were performed to identify the FA profile of the residue and distillate fractions obtained in the second distillation at 135,145 and $155^{\circ} \mathrm{C}$. No significant differences were observed in the FA profile of the residue fractions at these temperatures. The FA profile was found to be in the range of $28-30 \%$ myristic and palmitic acids as MC-SFA, and $24-28 \%$ of the sum of EPA, DPA and DHA as LC-PUFA. However, despite the similar purity of MAG found in the distillate fractions (around 90\% of MAG in the three cases, as shows Fig.4.2B), there was a clear difference in their FA profiles, as can be seen in Fig. 4.4A. The distillate obtained at $135^{\circ} \mathrm{C}$ contained almost $40 \%$ of MC-SFA (C14:0 and C16:0), while the $155{ }^{\circ} \mathrm{C}$ distillate contained only $23 \%$ of these fatty acids and more than $31 \%$ of LC-PUFA. It means that low $T_{E}$ is more suitable to obtain a distillate rich in MC-SFA. On the other hand, the content of n-3 LC-PUFA (EPA, DPA and DHA) increased in the distillate at high $\mathrm{T}_{\mathrm{E}}$. According to these results, a third distillation was carried out to 
remove MAG with MC-SFA in the D stream, while concentrating MAG with LC-PUFA in the $\mathrm{R}$ fraction, using fraction with more than 90\% MAG as feeding material (D2 in Fig. 4.3). The distillation was run at $125{ }^{\circ} \mathrm{C}$ as $T_{E}$. As shown in Fig. 4.4A, the distillation step resulted in almost $50 \%$ of MC-SFA and only around $10 \%$ of n-3 LC-PUFA in D fraction. Accordingly, the most of the EPA, DPA and DHA were remained in the $\mathrm{R}$ fraction after distillation at $125^{\circ} \mathrm{C}$.

A complete FA profile of $\mathrm{R}$ and $\mathrm{D}$ fractions collected in the third distillation is represented in Fig. 4.4B. The main differences between the FA compositions of the two streams were found in C14:0, EPA and DHA. In this way, almost all C14:0 was concentrated in the D fraction and on the contrary, EPA and DHA were found predominantly in the R fraction. In the case of the series of C18 FA with double bonds, as well as C18:0, no important differences between the streams were observed, because their concentration in the starting sardine oil was low. In summary, these results demonstrated that the third distillation process at $125^{\circ} \mathrm{C}$ contributed to the recovery of $n-3$ LC-PUFA in the residue. However, the level of concentration is highly affected by initial content of these FA (Kahveci \& Xu 2011). Further experiments with high initial content of n-3 LC-PUFA in the substrate should be done to achieve higher yield and purity of MAG with n-3 LC-PUFA.

\section{Conclusions}

This work reported the optimization of an SPD process for separating a mixture of acylglycerols, free fatty acids and glycerol, obtained from an enzymatic glycerolysis of sardine oil, which produced high concentration of MAG (67\%). The products (TAG, DAG, MAG, and FFA) contain n-3 PUFAs, and SPD is a suitable process to preserve their stability. A two-step distillation was applied to remove firstly at $110^{\circ} \mathrm{C}$ as $T_{E}$ the glycerol and part of FFA (63.5\%), and then a second distillation at $155^{\circ} \mathrm{C}$ produced a distillate with a MAG purity of 91 and $5.7 \%$ of DAG and with more than $31 \%$ of $n-3$ PUFA, which might be the emulsifier for some specific uses. This work further demonstrated that a third distillation process at $125{ }^{\circ} \mathrm{C}$ could contribute to the concentration of omega-3 LC-PUFA as MAG form in the residue. However, high initial content of $n-3$ LC-PUFA in the substrate is required to achieve higher yield and purity of MAG with n-3 LC-PUFA. 

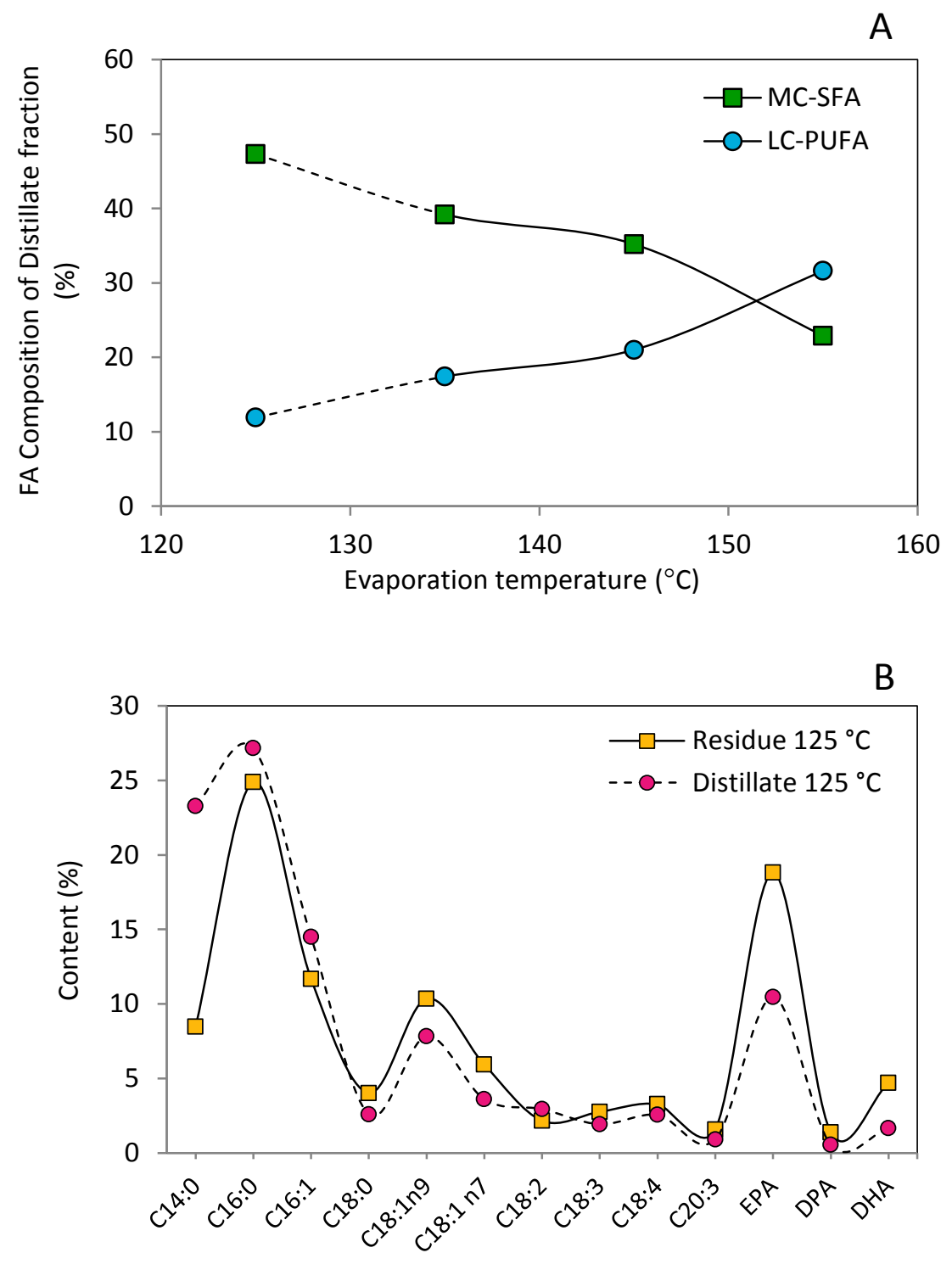

Fatty acid

Fig. 4.4. (A) Fatty acid composition of distillate fractions from the second distillation at 135,145 and $155^{\circ} \mathrm{C}$, and with MAG fraction as the feed material at $125{ }^{\circ} \mathrm{C}$, MC-SFA included C14:0 and C16:0, while LC-PUFA included EPA, DPA and DHA. (B) Comparison of the fatty acid profiles of the residue and distillate fractions at $125^{\circ} \mathrm{C}$. 


\section{CHAPTER 5}

\section{Substrates emulsification process to improve lipase-catalyzed sardine oil glycerolysis in different systems. Determination of lipid oxidation of the reaction products}

Á. García Solaesa, M.T. Sanz, R. Melgosa and S. Beltrán (2017).

"Substrates emulsification process to improve lipase-catalyzed sardine oil glycerolysis in different systems. Determination of lipid oxidation of the reaction products"

Food Research International. Article in Press. Available online 21 July 2017 



\title{
Capítulo 5
}

\author{
Proceso de emulsificación de los sustratos para mejorar la \\ glicerolisis de aceite de sardina catalizada por una lipasa en \\ diferentes sistemas. Determinación de la oxidación lipídica de \\ los productos de reacción.
}

\section{Resumen}

Como ya se ha visto en los capítulos anteriores, el principal inconveniente en la producción de MAG y DAG por glicerolisis es la inmiscibilidad de los sustratos, glicerol y aceite. Para mejorar las velocidades de transferencia de materia evitando el uso de disolventes orgánicos, en este capítulo se aborda la emulsificación de los sustratos como micelas reversas (glicerol en aceite) previa a la glicerolisis enzimática de aceite de sardina La emulsificación de los sustratos dio lugar a mayores velocidades de reacción que en las cinéticas sin emulsificación previa, aunque estas fueron más lentas que con los tertalcoholes como medio de reacción obtenidas en los capítulos anteriores. También en este capítulo se abordó la adición de surfactantes, Tween 80 y AOT, al sistema; así como el uso de $\mathrm{CO}_{2}$ supercrítico $\left(\mathrm{CO}_{2}-\mathrm{SC}\right)$ como medio de reacción. Sin embargo no se observaron ventajas significativas en las cinéticas de reacción en ningún caso, ni en las velocidades iniciales ni en el rendimiento. Por otro lado se estudió también el estado de oxidación de los productos de reacción, y se comprobó que un aumento de la temperatura de reacción no sólo se asociaba con un aumento en las velocidades iniciales de reacción; si no que no conllevaba un aumento en la oxidación final de los productos de reacción, si no todo lo contrario. Lipozyme 435 tiene la capacidad de adsorber los compuestos de oxidación, siendo esta capacidad mayor a mayor temperatura. 



\section{Abstract}

Mono- and diacylglycerols rich in omega-3 have a great interest due to their good bioavailability and oxidation stability compared with other kind of omega-3 concentrates. The main drawback in mono- and diacylglycerols production by glycerolysis is the immiscibility of the substrates, oil and glycerol. To improve mass transfer rates, avoiding the use of organic solvents, emulsification of both reactants as reverse micelles (glycerolin-oil) was carried out previous to lipase-catalyzed sardine oil glycerolysis. Substrate emulsification yielded higher reaction rates compared to kinetics with no previous emulsification, but still lower than in organic solvents. To avoid the use of organic solvents, $\mathrm{SC}-\mathrm{CO}_{2}$ was used as reaction medium but no kinetic advantages were demonstrated in the pressure range from 15 to $25 \mathrm{MPa}$. By increasing temperature, from 40 to $90^{\circ} \mathrm{C}$, reaction rates increased both in a solvent-free system and in SC-CO medium. It was also found that an increase in temperature does not lead to an increase in the final oxidation status of the reaction products. This behavior was due to the adsorption capacity of the Lipozyme 435 support, giving lower oxidation status at the highest temperature, $80-90{ }^{\circ} \mathrm{C}$

\section{Introduction}

The importance of omega-3 polyunsaturated fatty acids ( $n-3$ PUFA), especially eicosapentaenoic acid (EPA) and docosahexaenoic acid (DHA), in human nutrition and disease prevention is fully recognized scientifically (Kris-Etherton et al. 2002; Riediger et al. 2009). n-3 PUFA supplements are available in different chemical forms. Among the different types of lipid derivatives, monoacylglycerols (MAG) and diacylglycerols (DAG) have good bioavailability and oxidation stability (Hernandez 2014; Lawson \& Hughes 1988b). Additionally, it must be also considered that dietary TAG are hydrolyzed in the small intestine to sn-2-MAG being the most favorable structure for $n-3$ PUFA to be absorbed by intestinal mucosa (Van Dijk et al. 2012). In addition, MAG or its mixtures with DAG account for $75 \%$ of the worldwide emulsifier production (Zhong et al. 2009). In industry, glycerolysis is carried out by inorganic alkaline catalysis at high temperatures $\left(220-260^{\circ} \mathrm{C}\right)$ (Solaesa, Sanz, Beltrán, \&Melgosa, 2016). The well-known drawbacks of this 
technique (energy intensive, low yields (30-40\%), oxidized products) have prompted a growing interest in the development of alternative processes for the production of MAG and DAG rich in n-3 PUFA. Enzyme-catalyzed reaction is an attractive alternative since the reaction can be carried out under mild conditions (Bornscheuer 1995; Feltes et al. 2013).

To overcome the problem of the immiscibility of glycerol and oil, different approaches have been used in the literature to improve the contact between the reactants and hence reduce mass transfer limitation. Lipase-catalyzed glycerolysis has been carried out in different reaction media such as organic solvents (Damstrup, Jensen, et al. 2006), compressed fluids (Moquin et al. 2005) and ionic liquids (Guo \& Xu 2006), in order to improve the mass transfer. The cost, toxicity and energy required for solvent removal from the product mixture, are important aspects to be considered when dealing with conventional solvent systems (Prat et al. 2014). Recently, the uses of different surfactants to increase the interfacial area, and ultrasound irradiation have been also proposed to reduce mass transfer limitation (Fiametti et al. 2012; Valério et al. 2010). Biocatalytic processing in microemulsion system has received attention in order to increase contact between substrates. The formation of a microemulsion of the reactants (glycerol-in-oil) as reverse micelles can help to improve mass transfer rates. Furthermore, lipases demonstrate high interfacial activity in micelle systems because the formation of the active site during the reaction occurs at the interface between the substrates and the enzyme. Several food grade surfactants are able to stabilize the micellar system improving system homogeneity (Carvalho \& Cabral 2000; Stamatis et al. 1999). Nevertheless, it must be taken into account that some food grade surfactants have chemical functions that could be modified by lipases. For instance, the lipase Novozym 435 presented activity at particular conditions towards some surfactants as soy lecithin and Tween in glycerolysis reactions (Feltes et al. 2012). To avoid this problem, other synthetic surfactants, such as sodium (bis-2-ethyl-hexyl) sulfosuccinate (aerosol-OT or AOT), have been used. AOT has been reported to form micelles in a great number of nonpolar substances and several other polar solvents such as glycerol (Fiametti et al. 2009). Good results have been obtained in glycerolysis systems when adding more that $7.5 \%$ of AOT (Fiametti et al. 2009). However, the high amount of this surfactant may generate problems during removal processes (Stamatis et al. 1994). 
Another alternative to organic solvents is the use of the supercritical fluids (SCFs) as reaction medium. Supercritical carbon dioxide $\left(\mathrm{SC}-\mathrm{CO}_{2}\right)$ is probably the most used SCF due to its additional benefits (non-toxic, non-flammable, readily available at high purities and low costs, and relatively mild critical conditions) that are appealing when choosing environmental replacement for organic solvents (Matsuda 2013; Rezaei et al. 2007). SC$\mathrm{CO}_{2}$ has liquid-like density but gas-like viscosity resulting in high mass transfer being a clean alternative to replace organic solvents. Enzymatic concentration of $n-3$ PUFA in supercritical fluids (SCFs) is an interesting option for the prevention of oxidation during processing of fish oil (Lin et al. 2006; Roh et al. 2015). Besides, SC- $\mathrm{CO}_{2}$ can be easily separated from the reaction products by simple depressurization and allows fractionation of the reaction products. Some previous studies of enzymatic reactions of different lipid sources in $\mathrm{SC}-\mathrm{CO}_{2}$ have been reported in the literature. However, in case of enzymatic glycerolysis, other compressed fluids such as propane, n-butane, and acetone, have been used (Esmelindro et al. 2008; Tai \& Brunner 2011; Valério et al. 2010). Some studies of glycerolysis of vegetable oils in $\mathrm{SC}-\mathrm{CO}_{2}$ at high temperatures can be found but with no enzymatic catalyst (Moquin et al. 2005; Temelli et al. 1996).

In a previous work, a detail kinetic study of the glycerolysis of sardine oil using Lipozyme 435 from Candida antarctica B as biocatalyst in tert-butanol was performed (Solaesa, Sanz, Beltrán, et al. 2016). Tert-butanol helped to create a homogeneous phase and reduce mass transfer limitations. However, organic solvents present different environmental concerns. To improve contact between substrates, avoiding the use of organic solvents, emulsification of glycerol and oil before glycerolysis reaction was considered in this work. Glycerolysis reaction has been performed in a solvent free system at atmospheric pressure and in $\mathrm{SC}-\mathrm{CO}_{2}$ as reaction medium with previous substrates emulsification. The effect of adding a surfactant, AOT or Tween 80 , to stabilize the emulsion, on glycerolysis performance has been also studied. Glycerolysis has been determined at different operating temperatures at atmosphere pressure, $0.1 \mathrm{MPa}$, and in $\mathrm{SC}-\mathrm{CO}_{2}$ medium in the pressure range from 15 to $25 \mathrm{MPa}$. Since $\mathrm{n}-3$ PUFA are highly susceptible to oxidation; the oxidative status of the final reaction products was evaluated through the peroxide and anisidine values. Reaction yields of the reaction products 
obtained in this work were compared with previous results obtained in tert-butanol as reaction medium

\section{Experimental section}

\subsection{Materials}

Refined sardine oil was provided by Industrias Afines S.L. (Spain) with $18.3 \%$ of EPA and $7 \%$ of DHA and a water content of $0.2 \%$ (Solaesa et al. 2014). Glycerol was purchased from Sigma Aldrich with a purity of $\geq 99.5 \%$ and a water content of $0.18 \%$. The food grade lipase Lipozyme 435 from Candida antarctica B (immobilized on a macroporous hydrophobic acrylic resin), was donated by Novozymes A/S (Bagsvaerd, Denmark). Carbon dioxide (99.9\%) was supplied by Air Liquide S.A. (Spain). Polyoxyethylene sorbitan monooleate (Tween 80) and sodium bis (2-ethylhexyl) sulfosuccinate (Aerosol AOT or AOT), used as food grade surfactants, were purchased by Sigma Aldrich. All other chemicals used in different analyses were of analytical or HPLC grade.

\subsection{Emulsification process}

Microemulsions of the glycerolysis system of sardine oil were prepared at a fixed mole ratio of 3:1 (glycerol:oil) since this mole ratio was found as the optimum in a previous kinetic study (Solaesa, Sanz, Beltrán, et al. 2016). A high-speed blender (Miccra D9 equipped with a DS-20/PF EMR rotor-stator) at different speeds, from 16000 to 35000 rpm, was used by pulses during 3 minutes. To prepare the surfactant-free emulsion as reverse micelles, the appropriate amount of glycerol (10 g) was added drop by drop to the suitable amount of oil (30 g) while being completely mixed at high speed. Reverse micelles formation was confirmed by the dilution test (Davies 1957). Furthermore, different concentrations $(0.5,1$ and $1.5 \%$ in glycerol or oil as indicated in Table 5.2) of two food grade surfactants, AOT and Tween 80 , were tested in order to improve the stability of the emulsion. The characterization of the emulsions was performed $10 \mathrm{~min}$ after emulsification to avoid any creaming or coalescence effect. Dispersed (glycerol) and continuous (sardine oil) phases were identified in the emulsions formed in this work by the dilution test (Mize et al. 2013). Particle size distribution (PSD), mean droplet diameter 
and polydispersity index (PDI) of samples were measured by dynamic light scattering (DLS), using a Zetasizer Nano ZS apparatus (Malvern Instruments Ltd., UK) to evaluate the best conditions to produce a stable emulsion with small (or the smallest) droplet size.

\subsection{Lipase-catalyzed glycerolysis of sardine oil in different systems}

A comparative study of lipase-catalyzed glycerolysis in different systems was carried out. All the experiments were conducted in a batch mode keeping constant the enzyme concentration at $5 \mathrm{wt} \%$ (by weight of substrates) and the substrate mole ratio (MR) (3:1, glycerol to oil) according to previous work (Solaesa, Sanz, Beltrán, et al. 2016). Table 5.1 summarizes all glycerolysis reactions that have been done in this work. Experiments 1 - 6 have been carried out in a solvent free system at atmospheric pressure in a $100 \mathrm{~mL}$ jacketed batch reactor. First of all, experiments 1 and 2 were carried out to evaluate the effect of previous substrates emulsification on reaction rate. Experiments 3 and 4 were performed with emulsified substrates stabilized by adding a food grade surfactant, AOT and Tween 80 respectively, at the optimum concentration previously determine in section 2.2. Experiments 2, 5 and 6 were performed to evaluate the effect of reaction temperature, 50, 80 and $90^{\circ} \mathrm{C}$ respectively. Glycerolysis reaction was carried out as follows. Once emulsion was prepared, it was charged into the reactor. Later, the lipase was added and a nitrogen stream was applied. The reactor was then closed and the stirring system by impellers was connected. A thermostatic water bath allows working at the desired temperature. The reactor was covered with foil paper to avoid the light exposure.

On the other hand, experiments 7-13 have been carried out in $\mathrm{SC}-\mathrm{CO}_{2}$ as reaction medium. Kinetics were performed in a high pressure batch stirred tank reactor made of stainless steel, having an internal volume of $100 \mathrm{~mL}$ (Melgosa et al. 2017). A freshly prepared emulsion and the lipase were charged into the reactor with magnetic agitation and then it was closed, placed in a thermostatic water bath and connected to the pressure circuit. Subsequently, $\mathrm{SC}-\mathrm{CO}_{2}$ was fed into the reactor by means of a high pressure pump (ISCO $260 \mathrm{D})$ up to the desired pressure, which was maintained by a digital pressure controller. 
Operating pressure and temperature have been varied in the range between $15-25 \mathrm{MPa}$ (Exp.7-9) and $40-90^{\circ} \mathrm{C}$ (Exp. 7 and 10-13), respectively.

In both systems, samples were taken periodically during $8 \mathrm{~h}$, filtered and stored at $-18^{\circ} \mathrm{C}$ up to analysis.

Table 5.1. Summary of the reaction conditions for lipase-catalyzed sardine oil glycerolysis reactions carried out in this work.

\begin{tabular}{ccccc} 
Exp. & $\begin{array}{c}\text { Reaction } \\
\text { medium }\end{array}$ & $\begin{array}{c}\text { Pressure } \\
(\mathrm{MPa})\end{array}$ & $\begin{array}{c}\text { Temperature } \\
\left({ }^{\circ} \mathrm{C}\right)\end{array}$ & $\begin{array}{c}\text { Emulsification } \\
1\end{array}$ \\
2 & & 0.1 & 50 & No \\
3 & Solvent & 0.1 & 50 & Yes \\
4 & free & 0.1 & 50 & Yes $^{1}$ \\
5 & & 50 & Yes $^{2}$ \\
6 & & 0.1 & 80 & Yes \\
\hline 7 & & 15 & 90 & Yes \\
8 & & 20 & 50 & Yes \\
9 & SC-CO & 25 & 50 & Yes \\
10 & as & 15 & 40 & Yes \\
11 & solvent & 15 & 65 & Yes \\
12 & & 15 & 80 & Yes \\
13 & & 15 & 90 & Yes \\
\hline
\end{tabular}

${ }^{1}$ AOT as surfactant, ${ }^{2}$ Tween 80 as surfactant

\subsection{Analysis of the reaction products}

The neutral lipid profile (TAG, DAG, MAG and FFA) was analyzed by a normal phase high performance liquid chromatography (NP-HPLC). The chromatographic apparatus consisted of a HPLC system (Agilent 1200) formed by a quaternary pump and an auto-injector. The chromatographic separation of the compounds was carried out at room temperature with a Lichrospher Diol column $(5 \mu \mathrm{m}, 4 \mathrm{~mm} \times 250 \mathrm{~mm}$ ) and detection was performed by an 
evaporative light scattering detector (Agilent 1200 series) at $35^{\circ} \mathrm{C}$ and $0.35 \mathrm{MPa}$. Gradient elution was achieved by mobile phases A (isooctane) and B (methyltert-butyl ether:acetic acid $=99.9: 0.1, v / v)$. The method and calibration procedure have been previously reported (Solaesa, Sanz, Falkeborg, et al. 2016). The regioisomers of DAG and MAG could not be distinguished by the applied analytical procedure, so the total amount of MAG and DAG was reported for the kinetic experiments. The lipid profile results were expressed in glycerol free basis.

\subsection{Lipid oxidation analysis}

The oxidation status has been determined using two assays: peroxide value (PV) and anisidine value (AV). The PV measures the concentration of hydroperoxides formed in the initial stages of lipid oxidation (primary oxidation). PV was determined following the AOAC Official Method 965.33 by an automatic titrator Methrom 905 Titrando (AOAC Official Method 965.33 2000). The AV is an estimation of the concentration of non-volatile secondary oxidation products (mainly 2-alkenals and 2,4-dienals). The AV was measured according to AOCS official method (Cd 18-90), using a UV-Visible spectrophotometer (AOCS Official Method Cd 18-90 2017). PV and AV allow calculating total oxidation (TOTOX) by the formula:

TOTOX $=2 \mathrm{PV}+\mathrm{AV}$

PV and AV have been determined for the supplied refined sardine oil and the final reaction mixtures obtained after $8 \mathrm{~h}$ at the different temperatures assayed. The lipid phase was separated for analysis from the lipase and the remained glycerol by centrifugation at $5000 \mathrm{rpm}$ and $35^{\circ} \mathrm{C}$ during 10 minutes. The upper phase, free of glycerol, formed by the lipid fraction (TAG, DAG, MAG and FFA) was collected under $\mathrm{N}_{2}$ atmosphere and stored at $-18^{\circ} \mathrm{C}$ up to analysis.

\subsection{Statistical analysis}

All analyses were conducted using software Statgraphics X64. The results are presented as a mean \pm standard deviation of at least three replicates. The significance of the differences 
was determined based on an analysis of the variance with the Tukey's honestly significant difference (HSD) method at $p$-value $\leq 0.05$.

\section{Results and discussion}

\subsection{Optimization of the emulsification process and characterization of the emulsion}

\section{$\underline{\text { Surfactant-free emulsions }}$}

The effect of emulsification speed on emulsion stability without the addition of surfactants has been evaluated by measuring the polydispersity index (PDI) and the droplet diameter of the emulsion obtained in the range from 16000 to $35000 \mathrm{rpm}$. At any of the emulsification speeds essayed, droplet diameter was lower than $2 \mu \mathrm{m}$ but 29000 rpm were needed to obtain a PDI below 1 . The lowest polydispersity index was obtained at the highest speed assayed in this work, $35000 \mathrm{rpm}$; however, foaming was observed. Therefore $29000 \mathrm{rpm}$ was selected for further substrate emulsifications. At this speed the mean droplet diameter of the emulsion was $301 \pm 44 \mathrm{~nm}$ and the PDI around 0.4. The surfactant-free emulsion presented a PDI lower than 1 only up to 20 minutes, although at longer times, still a translucent and homogeneous system was visually observed. In any case, the emulsion was prepared and immediately used as reaction media.

\section{Surfactant stabilized emulsions}

The use of a surfactant was also tested in this work to improve the emulsion stability and reaction rates. Two food grade surfactants, AOT and Tween 80 , with hydrophilic lipophilic balance (HLB) values of 10 and 15 respectively, were used at different amounts $(0.5,1$ and 1.5\%). PDI of the emulsions prepared adding these surfactants were measured at specific times to evaluate their stability. Although a surfactant was added to stabilize the emulsions, PDI in the different emulsions increased with time in all cases (Table 5.2). The higher stability was observed when $0.5 \%$ of Tween 80 and $1.5 \%$ of AOT were previously dissolved in glycerol. In these cases the emulsions were found to be stable for at least $1 \mathrm{~h}$. PSD was evaluated for emulsion formed by adding $0.5 \%$ of Tween 80 and $1.5 \%$ of AOT in 
glycerol and compared with the PSD obtained for the surfactant-free emulsion. Smaller micelles were obtained when a surfactant was added to the system with medium particle sizes values of $67 \pm 5,94 \pm 2$ and $301 \pm 34$ for AOT $1.5 \%$ and $0.5 \%$ of Tween 80 dissolved in glycerol and surfactant-free emulsion, respectively.

Table 5.2. Polydispersity index (PDI) as measurement of reverse micelles stability when food grade surfactants, Tween 80 and AOT, are used at different concentrations dissolved in the glycerol phase $(\mathrm{G})$ or in the oil phase $(\mathrm{O})$ at different times.

\begin{tabular}{|c|c|c|c|c|}
\hline \multirow{2}{*}{ Surfactant } & \multirow{2}{*}{$\begin{array}{l}\text { Concentration of } \\
\text { surfactant (wt\%) }\end{array}$} & \multicolumn{3}{|c|}{ PDI after different times } \\
\hline & & $10 \mathrm{~min}$ & $30 \mathrm{~min}$ & $1 \mathrm{~h}$ \\
\hline \multirow{2}{*}{$\begin{array}{l}\text { Tween } 80 \\
(H L B=15)\end{array}$} & 0.5 in $\mathrm{G}$ & $0.17 \pm 0.04^{a b}$ & $0.29 \pm 0.01^{a}$ & $0.78 \pm 0.15^{b}$ \\
\hline & 1 in $\mathrm{G}$ & $0.63 \pm 0.09^{d}$ & $0.83 \pm 0.19^{b}$ & 1 \\
\hline \multirow{6}{*}{$\begin{array}{c}\text { AOT } \\
(H L B=10)\end{array}$} & 0.5 in $\mathrm{G}$ & $0.51 \pm 0.19^{c d}$ & $0.79 \pm 0.09^{b}$ & 1 \\
\hline & 1 in $G$ & $0.27 \pm 0.06^{\mathrm{ab}}$ & $0.50 \pm 0.01^{\mathrm{ab}}$ & 1 \\
\hline & 1.5 in G & $0.28 \pm 0.04^{\mathrm{abc}}$ & $0.45 \pm 0.13^{a}$ & $0.43 \pm 0.06^{a}$ \\
\hline & 0.5 in 0 & $0.07 \pm 0.02^{a}$ & $0.64 \pm 0.07^{a b}$ & 1 \\
\hline & 1 in 0 & $0.34 \pm 0.06^{\mathrm{bc}}$ & $0.43 \pm 0.03^{a}$ & 1 \\
\hline & 1.5 in 0 & $0.14 \pm 0.05^{\mathrm{ab}}$ & $0.43 \pm 0.09^{\mathrm{a}}$ & 1 \\
\hline
\end{tabular}

${ }^{\mathrm{a}} \mathrm{G}=$ dissolved in glycerol; $\mathrm{O}=$ dissolved in oil

Values with different letters in each column are significantly different when applying the Tukey's honestly significant difference (HSD) method at $p$-value $\leq 0.05$.

\subsection{Glycerolysis reaction of sardine oil by Lipozyme 435}

\section{Effect of substrates emulsification on the reaction rate}

Fig. 5.1 compares the kinetics of the glycerolysis reaction in a solvent free medium at atmospheric pressure with and without previous emulsification of the substrates (Exp 1 and 2 respectively). As it can be observed, when no previous emulsification of the reactants was carried out, mass transfer limitations lead to lower initial reaction rate. These limitations are reflected in the values of the initial slope of TAG composition as function of time being $0.15 \pm 0.01\left(\mathrm{~mol} \mathrm{TAG} \% \cdot \mathrm{min}^{-1}\right.$ ) without substrates emulsification 
and $0.279 \pm 0.008\left(\mathrm{~mol}\right.$ TAG $\left.\% \cdot \mathrm{min}^{-1}\right)$ with substrate emulsification. In a reverse micelle system, higher interfacial area is provided, which favors lipase-catalyzed reactions. At longer reaction times, reaction rates become similar due to the MAG and DAG formation that can act as emulsifiers. The low HLB values of MAG and DAG mean that they tend to stabilize reverse micelles systems (O'Brien 2004). Similar results were observed by Awadallak et al. (Awadallak et al. 2013) in the enzymatic palm oil hydrolysis under ultrasound irradiation to produce DAG. They also performed a control reaction (without ultrasound influence) to compare the degree of hydrolysis in both systems, being around $20 \%$ after $12 \mathrm{~h}$ in the control reaction and almost $40 \%$ when ultrasound was used before the reaction. But at longer reaction times $(24 \mathrm{~h})$ the degree of hydrolysis becomes similar. Therefore, they also concluded that ultrasound used before reaction to promote emulsification improved kinetics.

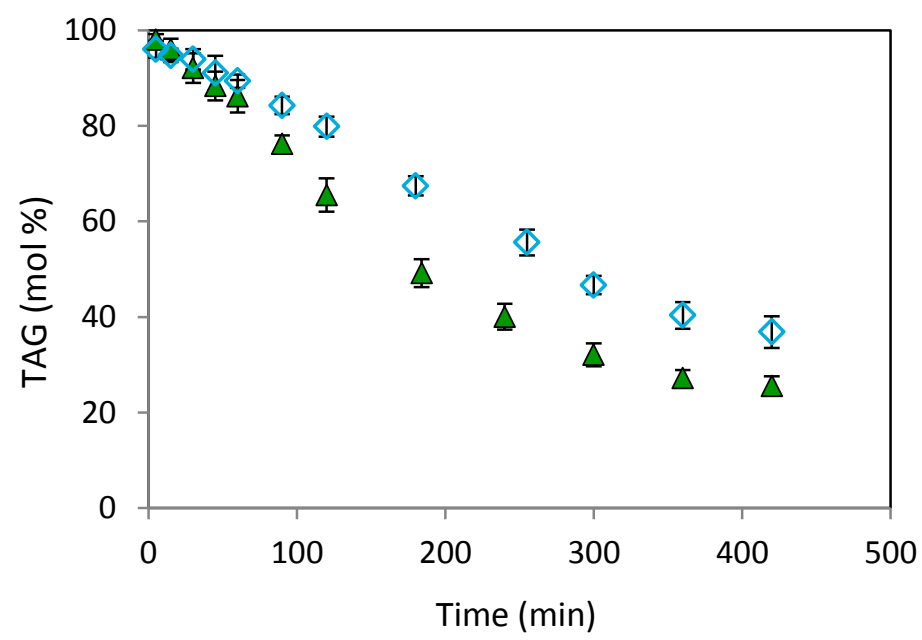

Fig. 5.1. TAG composition as a function of time in the glycerolysis of sardine oil in solvent free medium with $(\mathbf{\Delta})$ and without $(\diamond)$ substrates emulsification at atmospheric pressure (0.1 MPa). Reactions were performed at $\mathrm{MR}=3: 1$ (glycerol:oil), $\mathrm{T}=50^{\circ} \mathrm{C}$, enzyme loading $5 \%$ wt. of substrates.

Fig. 5.2 represents the reaction time course of TAG when surfactants, AOT and Tween 80, were added to the system at the concentration reported in Table $\mathbf{5 . 2}$ for the most stable emulsions, $1.5 \%$ of AOT and $0.5 \%$ of Tween 80 in glycerol. As previously shown, particle 
diameter was smaller when both surfactants, AOT or Tween 80 , were added to the emulsions; however this size reduction did not lead to significant improvement in the reaction rate or equilibrium conversion. Based on these results, further kinetics studies on glycerolysis were performed by previously emulsifying both reactants and no addition of surfactant.

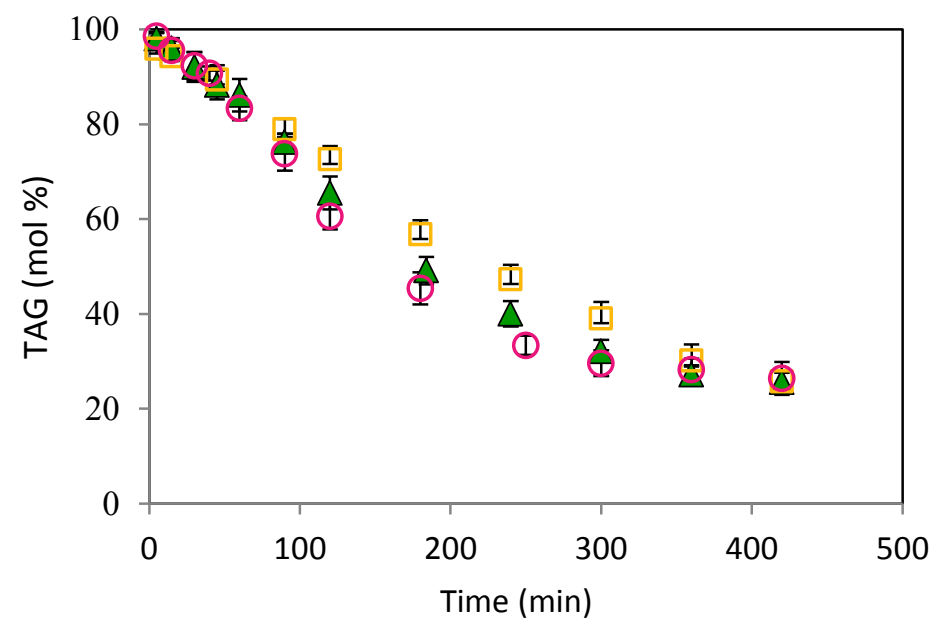

Fig. 5.2. TAG composition as a function of time in the glycerolysis of sardine oil previous substrates emulsification with $1.5 \%$ of AOT in glycerol ( $\square$ ), $0.5 \%$ of Tween 80 in glycerol (o) and surfactant free $(\boldsymbol{\Delta})$ at atmospheric pressure $(0.1 \mathrm{MPa})$. Reactions were performed at $\mathrm{MR}=3: 1$ (glycerol:oil), $\mathrm{T}=50^{\circ} \mathrm{C}$, enzyme loading $5 \%$ wt. of substrates.

In the literature, surfactants have been also used to improve the contact between substrates, however much higher concentration was employed. Fiametti et al. (Fiametti et al. 2009) evaluated AOT concentrations from 5 to $20 \%$ in the MAG production of olive oil, and obtained conversion values of around $60 \%$ for AOT concentration of 20 wt $\%$. On the other hand, Camino Feltes et al. (Feltes et al. 2012) employed 10\% of different surfactants (Tween 65, Tween 80, Tween 85 and soy lecithin) and they noticed that Tween as well as soy lecithin were partially modified by the lipase during the glycerolysis reaction. However, they acted as surfactants rather than as substrates at concentrations as low as $0.4-0.8 \%$. Additionally, the use of high amounts of surfactants may imply additional steps for the products recovery. 
In a previous work, lipase catalyzed glycerolysis have been performed in tert-butanol as organic reaction medium to avoid mass transfer limitations (Solaesa, Sanz, Beltrán, et al. 2016). Fig. $\mathbf{5 . 3}$ compares the glycerolysis reaction time course in a lipid base for TAG, DAG, MAG and FFA when tert-butanol was used as reaction media (Solaesa, Sanz, Beltrán, et al. 2016) with a solvent free media with previous substrate emulsification (Exp. 2). It can be observed that reaction rates are much higher in tert-butanol than in a free solvent media. This proves that mass transfer controls the reaction process since tert-butanol helps to create an homogeneous system but also to reduce the viscosity of the medium (viscosity of glycerol, fish oil and tert-butanol at $50^{\circ} \mathrm{C}$ are $142,20-30$ and $1.421 \mathrm{mPa} \cdot \mathrm{s}$, respectively). Due to mass transfer limitations, reaction rates are slower and the intermediate, DAG, accumulates in a greater extent in a solvent free media. After $7 \mathrm{~h}$ DAG accounts for $17.2 \%$, compared to less than $5 \%$ in tert-butanol. It seems that by controlling the viscosity of the medium selective formation of MAG and DAG can be obtained. In any case, although higher reaction rates and reaction yield were obtained in tert-butanol, organic solvents present a number of environmental concerns and its use should be avoided.

\section{$\underline{\text { Pressure effect }}$}

To avoid the use of organic solvents to improve the mass transfer and therefore increase glycerolysis reaction rate, reaction has been carried out in $\mathrm{SC}-\mathrm{CO}_{2}$ medium with previous substrates emulsification. Fig. 5.4 shows the effect of operating pressure (from 15 to $25 \mathrm{MPa}$ ) at $50^{\circ} \mathrm{C}$, on the reaction conversion and products yield after $7 \mathrm{~h}$ of reaction time. Based on these results it can be concluded that pressure has no significant effect on MAG and DAG yield or global TAG conversion in $\mathrm{SC}-\mathrm{CO}_{2}$ as reaction media in the pressure range studied in this work. TAG conversion ranged from 71 to $74 \%$ and MAG and DAG yield was 53 and $17 \%$, respectively. In this regard, the effect of pressure on enzyme catalysis in SC$\mathrm{CO}_{2}$ is difficult to predict since pressure affects the density and transport properties of SC$\mathrm{CO}_{2}$, but also has an effect on reaction rate since concentrations of reactants and products can be modified due to partitioning between the phases (Rezaei et al. 2007). 


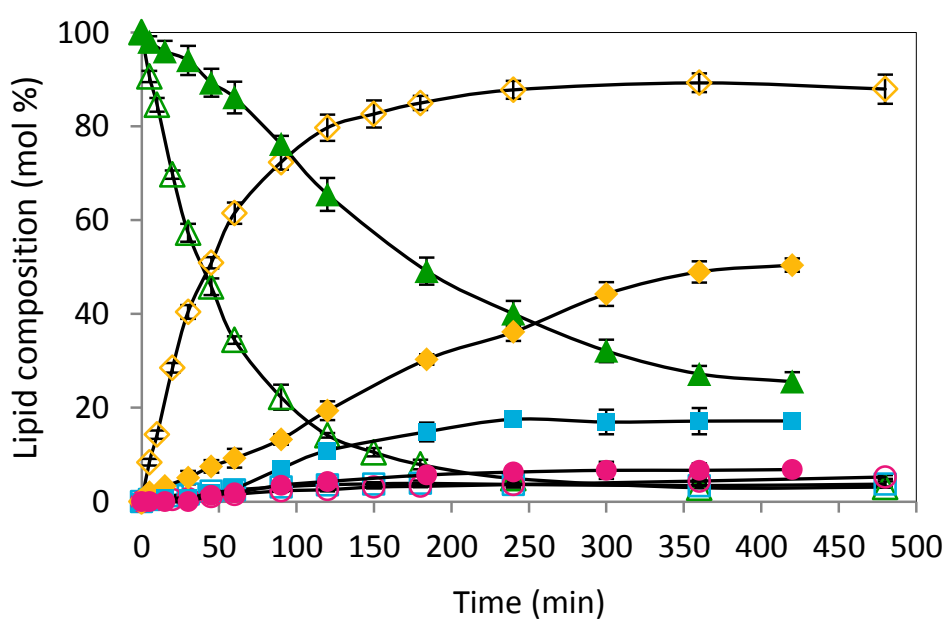

Fig. 5.3. Time course of lipase-catalyzed glycerolysis reaction of sardine oil in tert-butanol medium (hollow symbols) and in solvent free (solid symbols) with previous emulsification of the substrates at atmospheric pressure (0.1 MPa): TAG $(\Delta, \Delta), \operatorname{DAG}(\square, \square), \operatorname{MAG}(\diamond, \diamond)$ and FFA $(\bigcirc, \bullet)$. Reactions were performed at $M R=3: 1$ (glycerol:oil), $T=50^{\circ} \mathrm{C}$, enzyme loading $5 \%$ wt. of substrates.

In a reverse micelle system, sardine oil behaves as the continuous phase. Solubility of $\mathrm{CO}_{2}$ in fish oil increases with pressure at constant temperature, for instance, at $40^{\circ} \mathrm{C}$, solubility at $15 \mathrm{MPa}$ is $29.1 \%$ mass and at $25 \mathrm{MPa}$ is $33.1 \%$ mass (Borch-Jensen \& Mollerup 1997). This increase in solubility with pressure could improve the diffusivity in the reaction medium. However, fish oil solubility increases in $\mathrm{SC}-\mathrm{CO}_{2}$ by increasing pressure at constant temperature (Bucio et al. 2016). In the literature, it has been suggested (Temelli et al. 1996) that due to this solubility increment of TAG in the $\mathrm{SC}-\mathrm{CO}_{2}$, TAG could be in the supercritical phase, not being available in the liquid phase to react with glycerol that remains in the liquid phase due to its low solubility values in $\mathrm{SC}-\mathrm{CO}_{2}$ (Medina-Gonzalez et al. 2013; Nunes et al. 2013). These phenomenon's could cancel each other showing no effect of pressure on glycerolysis performance. Temelli et al. (Temelli et al. 1996) obtained similar results in the glycerolysis of soybean oil in $\mathrm{SC}-\mathrm{CO}_{2}$ at high temperatures (150$250^{\circ} \mathrm{C}$ ) in the pressure range from 20.7 to $62.1 \mathrm{MPa}$ and Tao et al. (Tao et al. 2013) in the enzymatic synthesis of dipalmitin from palmitic acid and glycerol at $65^{\circ} \mathrm{C}$ in the pressure range from 8.5 to $18.5 \mathrm{MPa}$ (Tao et al. 2013). Fig. 5.4 also presents the results obtained at atmospheric pressure at $50{ }^{\circ} \mathrm{C}$ in a solvent-free system with previous substrate 
emulsification (section 3.2.1). Lipid products profile was similar in both systems, $\mathrm{SC}-\mathrm{CO}_{2}$ and in a solvent-free media.

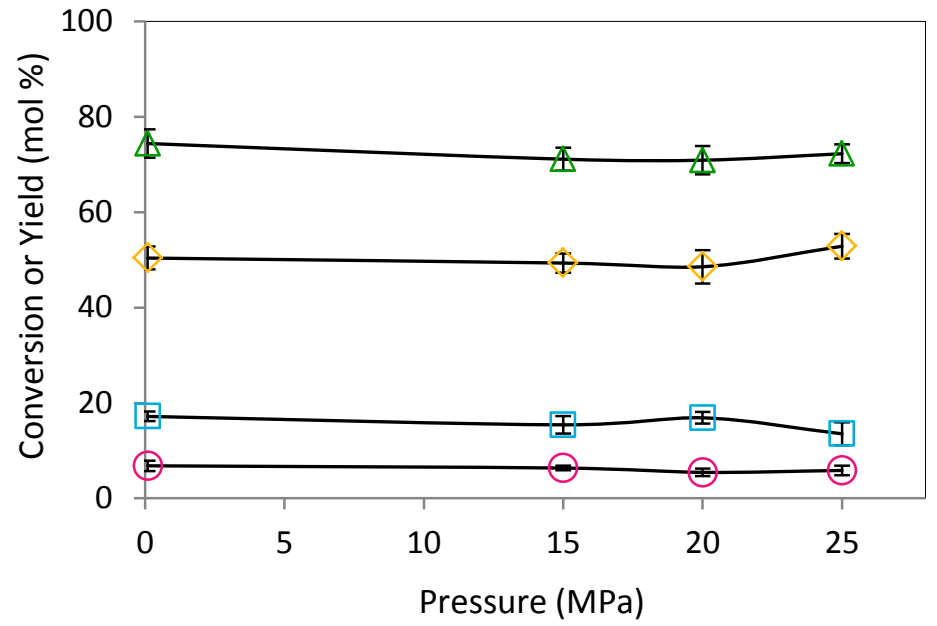

Fig. 5.4. Effect of pressure in SC-CO2 as reaction medium on glycerolysis conversion of sardine oil and products yields with previous substrates emulsification conversion of $\operatorname{TAG}(\Delta)$, yields of DAG, MAG and FFA ( $\square, \diamond$, and $\circ)$, respectively, after $7 \mathrm{~h}$ of reaction time. Results at atmospheric pressure in a solvent free system are also included (0.1 MPa). Reactions were performed at $\mathrm{MR}=3: 1$ (glycerol:oil), $\mathrm{T}=50^{\circ} \mathrm{C}$, enzyme loading $5 \% \mathrm{wt}$. of substrates.

To compare reaction rates, Table $\mathbf{5 . 3}$ summarizes the different initial reaction rates for TAG, DAG, MAG estimated by the initial slopes of the plot of the different lipid percentages profile as a function of time in both systems. Based on the properties of SC$\mathrm{CO}_{2}$, such as low viscosity and high diffusivity, kinetics are expected to be faster than in a solvent free system. However, due to the TAG solubility in $\mathrm{SC}-\mathrm{CO}_{2}$, fish oil is less available in the liquid phase to react with the glycerol. This could explain the similar results obtained at atmospheric pressure and in $\mathrm{SC}-\mathrm{CO}_{2}$. Therefore, from the reaction rate or product composition no advantages on the use of $\mathrm{SC}-\mathrm{CO}_{2}$ can be demonstrated. Although, it must be emphasized that the use of $\mathrm{SC}-\mathrm{CO}_{2}$ could be advantageous to the fractionation of the reaction products (Castillo et al. 1994). 


\section{Temperature effect}

To assess the effect of temperature on the kinetics of the glycerolysis of sardine oil by Lipozyme 435 in $\mathrm{SC}^{-} \mathrm{CO}_{2}$ media and in a solvent free system, operating temperature has been varied from 40 to $90^{\circ} \mathrm{C}$ (experiments $8,10-13$ ) and 50 to $90{ }^{\circ} \mathrm{C}$ (experiments 2,5 and 6), respectively. Initial substrate molar ratio (3:1 glycerol:sardine oil) and enzyme loading ( $5 \%$ wt. of substrates) remained unchanged. Fig. 5.5 shows the MAG + DAG production at different temperatures in $\mathrm{SC}_{-} \mathrm{CO}_{2}$. For a solvent free system, as it has been described in section "Pressure effect", the time reaction course overlap with those carried out in $\mathrm{CO}_{2}$ medium and it has not been represented. Equilibrium conversion is essentially temperature independent, although, at $40^{\circ} \mathrm{C}, 8$ hours was not sufficient time to achieve equilibrium concentration. Rising temperature from 40 to $90^{\circ} \mathrm{C}$ resulted in an increase of the initial reaction rate, due to the higher kinetic energy of the molecules that leads to lower viscosity and higher diffusivity of the solvent and substrates (Rezaei et al. 2007). It must be highlighted that enzyme activity was not negatively affected by temperature even at $90^{\circ} \mathrm{C}$.

Table 5.3. Initial slopes of TAG, DAG and MAG composition at different operating pressures previous substrates emulsification in $\mathrm{SC}-\mathrm{CO}_{2}$ (pressure range: $15-25 \mathrm{MPa}$ ) and at atmospheric pressure in a solvent free system $(0.1 \mathrm{MPa})$. Reaction conditions: $50^{\circ} \mathrm{C}, 5 \%$ enzyme loading based on substrate weight, $M R=3: 1$.

\begin{tabular}{lccc} 
Pressure, MPa & \multicolumn{3}{c}{ Initial slopes $\left(\mathrm{mol} \% \cdot \mathrm{min}^{-1}\right)$} \\
\hline $0.1^{*}$ & TAG & DAG & MAG \\
15 & $0.279 \pm 0.008^{\mathrm{a}}$ & $0.045 \pm 0.005^{\mathrm{a}}$ & $0.150 \pm 0.006^{\mathrm{a}}$ \\
20 & $0.296 \pm 0.010^{\mathrm{a}}$ & $0.052 \pm 0.002^{\mathrm{a}}$ & $0.202 \pm 0.007^{\mathrm{b}}$ \\
& $0.271 \pm 0.008^{\mathrm{a}}$ & $0.062 \pm 0.005^{\mathrm{a}}$ & $0.162 \pm 0.009^{\mathrm{a}}$ \\
& & & \\
& $0.284 \pm 0.012^{\mathrm{a}}$ & $0.052 \pm 0.008^{\mathrm{a}}$ & $0.204 \pm 0.009^{\mathrm{b}}$
\end{tabular}

* Solvent free.Values with different letters in each column are significantly different when applying the Tukey's honestly significant difference (HSD) method at $p$-value $\leq 0.05$. 
In both systems, $\mathrm{SC}-\mathrm{CO}_{2}$ medium and solvent-free, initial reaction rates followed an Arrhenius type dependence with temperature. From the slope of the Arrhenius plot, an estimation of the activation energy can be calculated as $52 \pm 2 \mathrm{~kJ} / \mathrm{mol}$ in SC-CO medium and a similar value for the solvent free system, $49 \pm 2 \mathrm{~kJ} / \mathrm{mol}$.

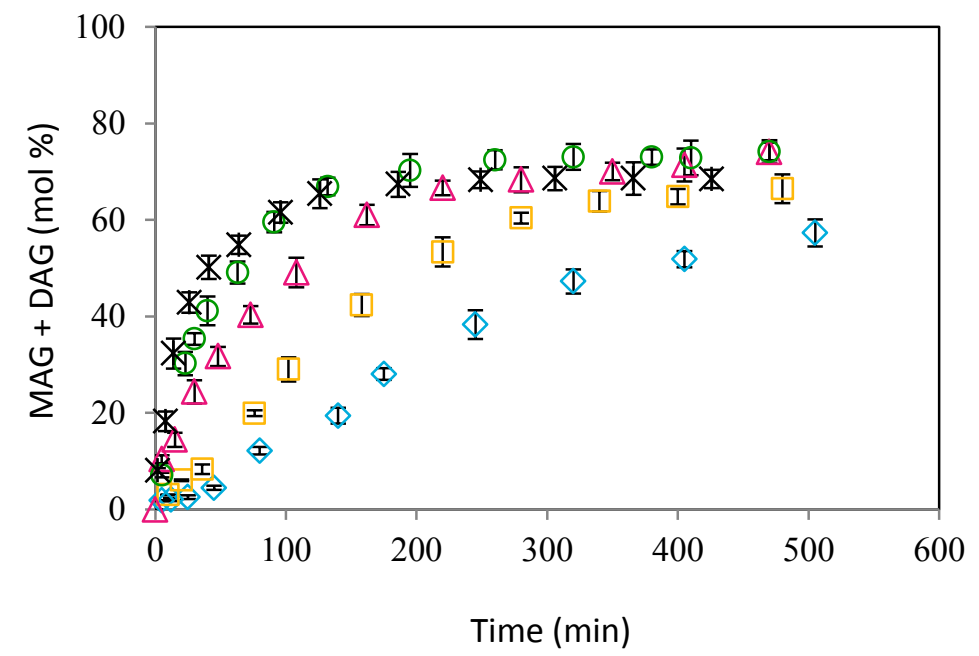

Fig. 5.5. Effect of temperature on MAG + DAG composition as a function of time in the glycerolysis of sardine oil with previous substrates emulsification in SC-CO2 at $15 \mathrm{MPa}$ : $40^{\circ} \mathrm{C}(\diamond), 50^{\circ} \mathrm{C}(\square), 65^{\circ} \mathrm{C}(\Delta), 80^{\circ} \mathrm{C}(\circ)$ and $90^{\circ} \mathrm{C}(\mathrm{x})$. Reactions were performed at MR = 3:1 (glycerol:oil) and enzyme loading $5 \%$ wt. of substrates.

\subsection{Comparison of lipid oxidation in both systems}

In section "Temperature effect" has been demonstrated that the enzyme Lipozyme 435 presents high thermal stability since the highest reaction rate has been obtained at the highest reaction temperature assayed in this work, $90^{\circ} \mathrm{C}$. However, when working with easily oxidizable compounds, such as n-3 PUFA, the oxidation status of the final reaction products must be taken into account. In this work, PV and AV have been determined for the refined sardine oil and the reaction mixtures obtained at the different temperatures after 7 hours of reaction in solvent free and in $\mathrm{SC}-\mathrm{CO}_{2}$ media. 
PV and AV for the supplied refined sardine oil were $4.8 \pm 0.1$ meq $\mathrm{O}_{2} / \mathrm{kg}$ and $23.0 \pm 0.1$ respectively. These values can be considered "acceptable" because they do not exceed the limit allowed (10 meq $\mathrm{O}_{2} / \mathrm{Kg}$ oil for $\mathrm{PV}$ and 30 for $\mathrm{AV}$ ) according to European Pharmacopeia Standard (European Pharmacopoeia 5.0 2005). Fig. 5.6 shows the PV and the $\mathrm{AV}$ of the reaction products obtained from 40 to $90{ }^{\circ} \mathrm{C}$. This figure shows unexpected results since PV decreased as reaction temperature increased, obtaining values of around 3 meq $\mathrm{O}_{2} / \mathrm{kg}$ at the highest temperatures assayed in this work $\left(80-90^{\circ} \mathrm{C}\right)$ which are even lower than that obtained for the supplied refined sardine oil. Similar results regarding oxidation status have been obtained in solvent free system in the temperature range from 50 to $90^{\circ} \mathrm{C}$. AV remained unchanged by increasing temperature (see Fig. 5.6).

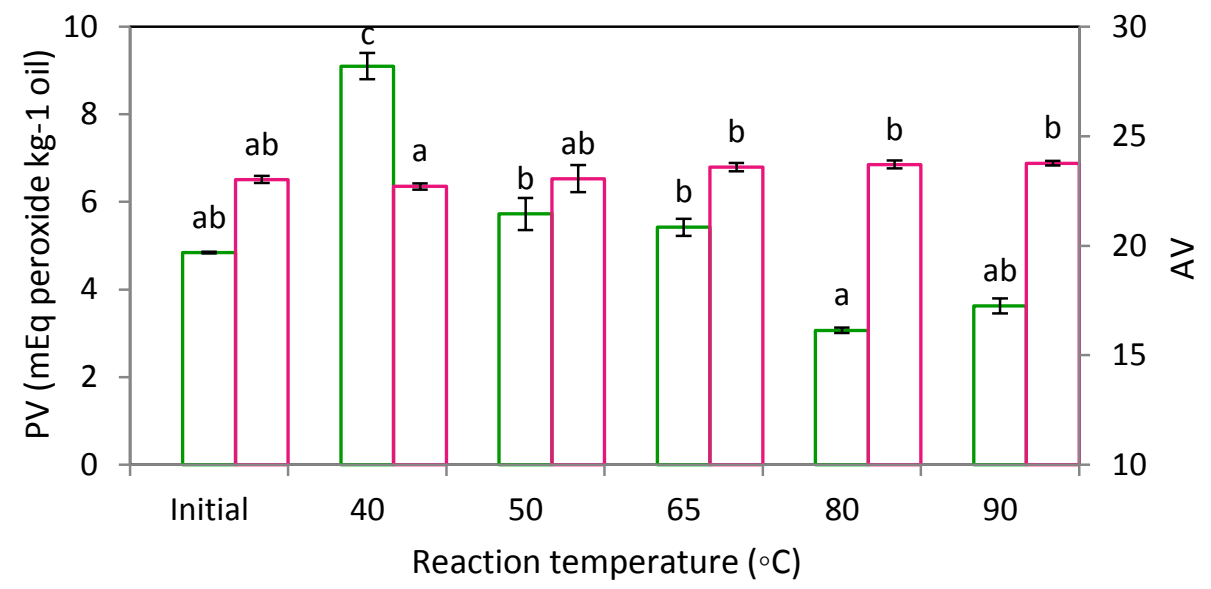

Fig. 5.6. Influence of reaction temperature on PV (green bars) and AV (pink bars) in the final reaction mixture after $7 \mathrm{~h}$ at $15 \mathrm{MPa}$ in $\mathrm{SC}-\mathrm{CO}_{2}$ as reaction medium with previous substrates emulsficiation. Measurements given are mean values based on four determinations. Limit allowed is the maximum of each axis. Values with different letters in each type of analysis (PV or AV) are significantly different when applying the Tukey's honestly significant difference (HSD) method at $p$-value $\leq 0.05$.

To determine the effect of temperature on autoxidation of sardine oil, oil samples were heated at 40,65 and $90^{\circ} \mathrm{C}$ for 7 hours and the oxidation status was analyzed. Table $\mathbf{5 . 4}$ shows that, both PV and AV are strongly depend on temperature, increasing as temperatures increased; however, the opposite trend was found in the oxidation status of the reaction products at the same temperatures. 
Table 5.4. PV, AV and TOTOX values obtained after 7 hours heating at different temperatures sardine oil and sardine oil in contact with Lipozyme 435 ( $28 \mathrm{~g}$ sardine oil : 1 g Lipozyme 435).

\begin{tabular}{llccccc} 
& \multicolumn{2}{c}{$\mathrm{PV}, \mathrm{meqO}_{2} / \mathrm{kg}$} & \multicolumn{2}{c}{ AV } & \multicolumn{2}{c}{ TOTOX } \\
$\mathrm{T}^{\mathrm{o}}{ }^{\mathrm{C}}$ & \multicolumn{1}{c}{ Sardine oil } & $\begin{array}{c}\text { Sardine oil } \\
+ \text { lipase }\end{array}$ & Sardine oil & $\begin{array}{c}\text { Sardine oil } \\
+ \text { lipase }\end{array}$ & Sardine oil & $\begin{array}{c}\text { Sardine oil } \\
+ \text { lipase }\end{array}$ \\
\hline 40 & $11.6 \pm 0.1^{\mathrm{a}}$ & $6.1 \pm 0.1^{\mathrm{c}}$ & $24.4 \pm 0.3^{\mathrm{a}}$ & $23.5 \pm 0.7^{\mathrm{a}}$ & $47.6 \pm 0.6^{\mathrm{a}}$ & $35.7 \pm 0.9^{\mathrm{a}}$ \\
65 & $22.3 \pm 0.2^{\mathrm{b}}$ & $4.8 \pm 0.1^{\mathrm{b}}$ & $30.2 \pm 0.8^{\mathrm{b}}$ & $25.5 \pm 0.6^{\mathrm{b}}$ & $75 \pm 1^{\mathrm{b}}$ & $35.1 \pm 0.8^{\mathrm{a}}$ \\
90 & $47 \pm 1^{\mathrm{c}}$ & $3.8 \pm 0.1^{\mathrm{a}}$ & $54.5 \pm 0.5^{\mathrm{c}}$ & $30.3 \pm 0.2^{\mathrm{c}}$ & $148 \pm 3^{\mathrm{c}}$ & $37.9 \pm 0.4^{\mathrm{b}}$ \\
\hline
\end{tabular}

Values with different letters in each column are significantly different when applying the Tukey's honestly significant difference (HSD) method at $p$-value $\leq 0.05$.

The lipase used in this work, Lipozyme 435, is immobilized on a macroporous hydrophobic acrylic resin; therefore it was assumed that peroxides could be adsorbed on the resin. To verify this assumption, sardine oil was mixed with the immobilized lipase at the same ratio sardine oil:lipase loading as for the glycerolysis reaction (28:1 g sardine oil:g lipase) and heated at 40,65 and $90^{\circ} \mathrm{C}$ for 7 hours under agitation. After that, PV and AV were determined. It can be clearly observed (see Table 5.4) that the amount of peroxides in the sardine oil decreased at each temperature when the immobilized lipase is present compared with the control sardine oil. Furthermore, lower peroxide values were obtained by increasing temperature, proving that sorption process of lipid peroxide in the enzyme support is favored by increasing temperature due to improved diffusivity. Regarding the $\mathrm{AV}$, lower values were also obtained in the sardine oil in contact with the immobilized lipase than in the sardine oil heated at the same temperature without lipase. Therefore, the support of the enzyme could also have capacity to adsorb secondary oxidation products. According to the PV and AV, TOTOX values also decreased in the sardine oil that has been in contact with Lipozyme 435 (Table 5.4). These results can explain the trend observed in the oxidation status of the reaction products with increasing temperature. In any case, it must be highlighted that during the glycerolysis reaction MAG and DAG are formed and sorption behavior could be modified. 


\section{Conclusions}

In this work, it has been demostrated that the emulsification of substrates before enzymatic glycerolysis reaction improved process efficiency in solvent free and in $\mathrm{SC}-\mathrm{CO}_{2}$ media, reducing mass transfer limitations in the three-phase system glycerol/oil/lipase, giving around $75 \%$ of MAG and DAG in 4 hours. Furthermore, when food grade surfactants, AOT or Tween 80, were added to the emulsified system, no significant improvement was observed neither in the reaction rate nor in the equilibrium conversions. It has been also found that pressure has no significant effect on reaction conversion and reaction rate, showing no kinetic advantages on the use of $\mathrm{SC}-\mathrm{CO}_{2}$ in glycerolysis reaction, although $\mathrm{SC}-\mathrm{CO}_{2}$ could be used to fractionate the reaction products. An increase in temperature from 40 to $90{ }^{\circ} \mathrm{C}$ produces higher reaction rates in both systems. Regarding the oxidation status of the reaction products, it has been concluded that higher reaction temperature results in a higher adsorption of the oxidation products on the support of the lipase, giving lower oxidation values. 



\section{CHAPTER 6}

\section{Effect of temperature on oxidation kinetics and adsorption capacity of commercial immobilized lipases supports to reduce oxidation products in sardine oil}

Á. García Solaesa, M.T. Sanz, R. Melgosa and S. Beltrán

"Effect of temperature on oxidation kinetics and adsorption capacity of commercial immobilized lipases supports to reduce oxidation products in sardine oil”

(Submitted in Food Research International) 



\title{
Capítulo 6
}

\author{
Efecto de la temperatura en las cinéticas de oxidación y \\ capacidad de adsorción de los soportes de las lipasas \\ comerciales inmovilizadas para reducir los productos de \\ oxidación en el aceite de sardina.
}

\begin{abstract}
Resumen
En este capítulo se ha estudiado la autooxidación del aceite de sardina a distintas temperaturasanalizando el valor de peróxidos como índice de oxidación primaria y el valor de anisidina y TBARS (sustancias reactivas al ácido tiobarbitúrico) como indicadores de oxidación secundaria. Debido al alto contenido en ácidos grasos poliinsaturados (AGPI) del aceite de sardina, la formación de hidroperóxidos y TBARS fue mayor que la de los compuestos que reaccionan con la anisidina, que aumentó más lentamente. Además, en este capítulo se ha evaluado la capacidad de adsorción de tres lipasas inmovilizadas comerciales (Lipozyme 435, Lipozyme RM y Lipozyme TL) por los compuestos de oxidación lipídica a distintas temperaturas. Las tres lipasas mostraron una buena capacidad de adsorción para reducir tanto los productos de oxidación primaria como secundaria formados a 65 y $90^{\circ} \mathrm{C}$, siendo más selectivas para los hidroperóxidos y TBARS que para los compuestos que reaccionan con la anisidina. Por lo tanto el uso de estas lipasas inmovilizadas en reacciones enzimáticas de aceites de pescado a temperaturas elevadas $\left(90^{\circ} \mathrm{C}\right)$ no sólo aumentará la velocidad de la reacción sino que también puede reducir los productos de oxidación que se formen.
\end{abstract}





\title{
Abstract
}

\begin{abstract}
Autoxidation of sardine oil has been studied at 40,65 and $90{ }^{\circ} \mathrm{C}$ by determining peroxide (PV), anisidine (AV) and thiobarbituric acid reactive substances (TBARS) values. The adsorption of these oxidation products have been evaluated by three commercial $j$ immobilized lipases (Lipozyme 435, Lipozyme RM and Lipozyme TL) at the same temperatures. These immobilized lipases have shown a good adsorption capacity to reduce oxidation products formed at the highest temperatures. Adsorption capacity has been attributed to the different enzyme supports. All supports seem to be more selective for hydroperoxides and TBARS than for anisidine reacted compound. The best adsorption capacity was observed for Lipozyme RM. Hence the use of these immobilized commercial lipases in lipase-catalyzed reactions of fish oils at high temperatures $\left(90^{\circ} \mathrm{C}\right)$ not only yields high reaction rates but also reduces the oxidation products formed due to their adsorption onto the enzyme support.
\end{abstract}

1. Introduction

Fish oil has high amounts of omega-3 long-chain polyunsaturated fatty acids ( $n-3$ PUFA), mainly eicosapentaenoic acid (EPA, 20:5n-3) and docosahexaenoic acid (DHA, 22:6n-3) which have been reported to protect against the development of many diseases, namely coronary heart diseases, hypertension and inflammatory disorders (Solaesa, Sanz, Falkeborg, et al. 2016). The consumers are becoming increasingly concerned about the nutritional value of n-3 PUFA. A popular way to increase n-3 PUFA intake is by available fish oils capsules and foods fortified with omega-3. These sources can be found both as purified fish oil and as concentrates in the form of triacylglycerols (TAG), mono and diacylglycerols (MAG and DAG), phospholipids, free fatty acids (FFA) and ethyl esters (EE) (Martín et al. 2012). These omega-3 concentrates can be produced by lipase-catalyzed reactions since they can be carried out under mild conditions in short reaction times compared with chemical process. Some widely used commercial immobilized lipases used in enzymatic reactions are from Candida antarctica (Novozym 435 and Lipozyme 435), Rhizomucor miehei (Lipozyme RM IM) and Thermomyces lanuginosus (Lipozyme TL IM) (Solaesa et al. 2015). Despite the high nutritional value of these products, it is well known 
that the high degree of unsaturation makes fish oils very prone to autoxidation, resulting in breakdown products that cause off-flavors and rancidity, loss of nutritional value and finally consumer rejection of the food product (Gómez-Alonso et al. 2004). The degree and rate of lipid oxidation is influenced not only by the unsaturation of fatty acids, but also by the oxygen concentration, temperature, surface area, water activity and presence of antiand prooxidants. PUFA are highly susceptible to oxidation since free radicals formed in the oxidation process are stabilized via delocalization of the unpaired electron (Kamal-Eldin \& Yanishlieva 2005). Temperature has also an important impact on lipid oxidation since an increase in temperature accelerates oxidation rates.

The process of lipid oxidation can be described in three general steps: initiation, propagation and termination. Hydroperoxides are the main primary oxidation products, accumulating during the initiation and propagation steps of the oxidation process. Decomposition of lipid hydroperoxides constitutes a very complicated process and produces a multitude of compounds. Hydroperoxides can form breakdown products, react again with oxygen and condense into dimers and polymers that can also breakdown and produce volatile materials. Among nonvolatile breakdown products, different compounds are formed such as aldehydes, ketones, epoxides, hydrocarbons, and others, that influence oil quality and stability (Frankel 1984). The concentrations of primary and secondary oxidation products can be measured quantitatively and therefore give an indication of the oxidative status of the oil. Peroxide value (PV) measures the hydroperoxides content as primary oxidation products. Secondary oxidation products can be determined by anisidine value (AV) and thiobarbituric acid reactive substances (TBARS) assay. AV measures mainly 2-alkenals and 2,4-dienals. These are acyclic unsaturated aldehydes containing at least one carbon-carbon double bond. On the other hand, the cyclic peroxides formed by autoxidation from polyunsaturated fatty acids with three or more double bonds are the most important precursors of malonaldehyde and hence source of TBARS (Hoyland \& Taylor 1991). Polyene index (PI) is also used as a good indicator of PUFA deterioration in fish oils, defined as (EPA + DHA): palmitic acid ratio (Pazhouhanmehr et al. 2016). 
The main challenge to produce high quality omega-3 concentrates is oxidation. To prevent oxidation and undesirable off flavours the industry has developed a wide variety of newantioxidants, encapsulation processes and other techniques to reduce its presence. Bleaching is one of the most important steps in edible oil refining to reduce the oxidation products and other undesirables compounds such as soaps, trace metals, pigments and phospholipids improving quality of the deodorized fish oil (Rossi et al. 2003). The adsorption process involves mass transfer of the adsorbates from the fluid phase to the adsorbent surface until the thermodynamic equilibrium of the adsorbates concentration is reached. The basic types of adsorbents used in edible-oil bleaching are neutral clays, activated earths, activated carbon, and synthetic amorphous silica (O'Brien 2004). In previous work (Solaesa et al. 2017), it was found that commercial immobilized lipase, Lipozyme 435, typically used in lipid modification reactions had also adsorption capacity for primary oxidation products in the temperature range from 40 to $90{ }^{\circ} \mathrm{C}$ where lipid modification reactions were performed. In that work it was found that primary oxidation products decreased in a greater extend at the highest reaction temperature essayed, $90^{\circ} \mathrm{C}$. Therefore an increase in temperature, not higher than the maximum denaturation temperature for the lipase, is positive since reaction rate increased and no increase of the final oxidation status was observed. Adsorption process in case of lipid oxidation products is the result of a balance between the formation, decomposition and adsorption of these products (Toro-Vazquez et al. 1991); therefore, the thermodynamic equilibrium results difficult to determine. Resins can adsorb hydroperoxides and catalyze their degradation into secondary oxidation products. Besides they can also have adsorption capacity for secondary oxidation products (Rossi et al. 2003). Moreover in case of immobilized lipases, not only the enzyme support may catalyze oxidation products formation or degradation, but also it could be possible that lipase, as a biocatalyst, could promote some oxidation reactions.

Based on those previous results, in this work, a systematic study of the adsorption capacity of three commercial immobilized lipases for adsorbing oxidation products formed in sardine oil during oxidation has been carried out in the temperature range where lipase catalyzed reactions usually take place. The commercial lipases used, immobilized onto different supports, were Lipozyme 435, Lipozyme RM and Lipozyme TL. To determine the 
adsorption capacity of the enzyme support, oxidation kinetics were first determined at the temperatures selected in this work $\left(40,65\right.$ and $\left.90^{\circ} \mathrm{C}\right)$ to know the net formation rate of the primary and secondary oxidation products. Afterwards, sardine oil was also incubated at these temperatures in contact with the immobilized lipases to evaluate the adsorption capacity of the lipases supports.

\section{Experimental section}

\subsection{Materials}

Refined sardine oil was provided by Industrias Afines S.L. (Spain). Three commercial immobilized lipases were used in this work. Lipozyme 435 from Candida antarctica B and Lipozyme TL IM from Thermomyces lanuginosus were kindly donated by Novozymes A/S (Bagsvaerd, Denmark). Lipozyme RM IM, from Rhizomucor miehei, was purchased in Sigma Aldrich. The support characteristics of these immobilized lipases are summarized in Table 6.1. All other chemicals used in the different analyses performed in this work, were of analytical or HPLC grade.

Table 6.1. Characteristics of the immobilized commercial lipases used in this work.

\begin{tabular}{|c|c|c|c|}
\hline Immobilized lipase & Support & Particle size & Reference \\
\hline $\begin{array}{c}\text { Lipozyme } 435 \\
\text { (Candida antarctica B) }\end{array}$ & $\begin{array}{l}\text { Macroporous resin of poly- } \\
\text { methyl methacrylate } \\
\text { (Lewatit VP OC 1600) }\end{array}$ & $315^{\sim} 1000 \mu \mathrm{m}$ & $\begin{array}{c}\text { (Chen et al. } \\
\text { 2008) }\end{array}$ \\
\hline $\begin{array}{c}\text { Lipozyme RM } \\
\text { (Rhizomucor miehei) }\end{array}$ & $\begin{array}{l}\text { Weak base anion-exchange } \\
\text { resin based on phenol- } \\
\text { formaldehyde copolymers } \\
\text { (Duolite ES 562) }\end{array}$ & $200 \sim 600 \mu \mathrm{m}$ & $\begin{array}{l}\text { (Rodrigues \& } \\
\text { Fernandez- } \\
\text { Lafuente } \\
\text { 2010) }\end{array}$ \\
\hline $\begin{array}{l}\text { Lipozyme TL } \\
\text { (Thermomyces } \\
\text { lanuginosus) }\end{array}$ & Granulated silica & $250 \sim 1000 \mu \mathrm{m}$ & (Zhang 2007) \\
\hline
\end{tabular}




\subsection{Oxidation kinetics of sardine oil and adsorption} kinetic experiments

Oxidation kinetics of sardine oil were evaluated at three different temperatures 40, 65 and $90{ }^{\circ} \mathrm{C}$. For each experiment, stoppered erlenmeyer glass flasks were filled with $70 \mathrm{~g}$ of refined sardine oil and immediately applied a nitrogen stream in the flask. Afterwards they were perfectly closed and covered with foil paper to avoid the light exposure. Sardine oil samples were incubated in a water bath with orbital agitation at the corresponding temperature. At different time intervals, during $10 \mathrm{~h}$, aliquots were withdrawn to follow the oxidation kinetics.

Adsorption experiments were carried out in a similar way, but in this case sardine oil was put in contact with the different immobilized commercial lipases, Lipozyme 435, Lipozyme RM and Lipozyme TL, that will act as adsorbents. The amount of immobilized lipase was fixed at $3.5 \% \mathrm{w} / \mathrm{w}$ in all the experiments. This concentration was found to be suitable for lipid modification reactions (Solaesa, Sanz, Falkeborg, et al. 2016). As for the oxidation kinetics, samples were withdrawal at different time intervals during $10 \mathrm{~h}$.

All samples were stored at $-18^{\circ} \mathrm{C}$ up to oxidation products analysis.Duplicate experiments were carried out at each temperature.

\subsection{Determination of primary oxidation products: peroxide value (PV)}

The PV measures the concentration of hydroperoxides formed in the initial stages of lipid oxidation (primary oxidation). PV was determined by iodometric titration following the AOAC Official Method (AOAC Official Method 965.33 2000)by an automatic titrator Methrom 905 Titrando. The reaction between a saturated solution of potassium iodide and an oil sample is the basis of the method. PV is expressed as milliequivalents of $\mathrm{O}_{2}$ per kilogram of sample. All samples were analyzed in triplicate. 


\subsection{Determination of secondary oxidation products}

\section{Anisidine value (AV)}

The method is based on the reaction between $\mathrm{p}$-anisidine and non-volatile secondary oxidation products (mainly 2-alkenals and 2,4-dienals) present in oil samples at acidic conditions producing yellow-colored compounds. The AV was measured according to AOCS Official Method (AOCS Official Method Cd 18-90 2017), using a UV-Visible spectrophotometer at $350 \mathrm{~nm}$. All samples were analyzed in triplicate.

\section{Thiobarbituric acid reactive substances (TBARS) assay}

The method is based on the formation of a pink complex with strong absorbance at 532 $535 \mathrm{~nm}$ when thiobarbituric acid (TBA) and malondialdehyde from decomposition of unsaturated fatty acids react. Because the reaction can involve several secondary oxidation products, the reacting secondary products are generally referred to as TBAreacting substances. TBARS were determined according to the spectrophotometric method described by Norveel Semb in her Master's Thesis (Norveel Semb 2012). TBARS is expressed as mg of malondialdehyde (MDA) per kilogram of oil. All samples were analyzed in triplicate.

\subsection{Fatty acid analysis}

The initial sardine oil and the oil samples after $10 \mathrm{~h}$ of incubation at 40,65 and $90^{\circ} \mathrm{C}$ were analyzed by the AOAC method (AOAC Official Method 991.39 2000) to evaluate the fatty acid profile and consequently the polyene index (EPA+DHA/16:0) as a good factor for determination of lipid oxidation in fishery products(Pirestani et al. 2010). An Agilent gas chromatograph (6890N Network GC System) equipped with a flame ionization detector (FID) and a fused silica capillary column (OmegawaxTM-320, $30 \mathrm{~m} \times 0.32 \mathrm{~mm}$ i.d.) was used. The method and the calibration procedure was developed previously (Solaesa, Sanz, Falkeborg, et al. 2016). The samples were analyzed in triplicate. 


\subsection{Statistical analysis}

Statistical analyses were conducted using software Statgraphics X64. The results are presented as a mean \pm standard deviation of at least three replicates. The significance of the differences was determined based on an analysis of the variance with the Tukey's honestly significant difference (HSD) method at $p$-value $\leq 0.05$. The estimation of the parameters for the model tested in this work was performed by using the Marquardt algorithm (Statgraphics X64).

\section{Results and discussion}

\subsection{Chemical quality of initial sardine oil}

The fatty acid profile and some quality parameters of the supplied refined sardine oil used in this work are presented in Table 6.2. The fatty acid composition reveals that sardine oil is a good source of PUFA (40\%), with $15.7 \%$ of EPA and $8.2 \%$ of DHA. The fatty acid profile is similar to those previously reported for sardine oil (Homayooni et al. 2014; Noriega-Rodríguez et al. 2009; Okada \& Morrisset 2007; Solaesa et al. 2014). The polyene index $(\mathrm{PI})$, evaluated as EPA+DHA/C16:0, of the supplied refined sardine oil used in this work is 1.39 (Table 6.2). This value is not significantly different to the PI value reported for sardine oil (Homayooni et al. 2014). Other important quality parameters such as acid value, $\mathrm{PV}$ and $\mathrm{AV}$ for the supplied refined sardine oil were $0.45 \mathrm{mg} \mathrm{KOH} / \mathrm{g}, 4.8 \mathrm{meq} \mathrm{O}_{2} / \mathrm{kg}$ and 19.7, respectively. These values are very close to the limits allowed by GOED Voluntary Monograph (GOED 2015), which are $0.5 \mathrm{mg} \mathrm{KOH} / \mathrm{g}$ for acid value, 5 meq $\mathrm{O}_{2} / \mathrm{kg}$ oil for PV and 20 for AV. Therefore, the supplied refined sardine oil used in this work is partially oxidized and although it can be still considered as "acceptable" oxidation kinetics could be faster due to the presence in the medium of oxidation products. Regarding TBARS, the maximum value expressed as $\mathrm{mg}$ of malondialdehyde (MDA)/kg of fish oil is not clearly specified. The TBARS levels of 5-8 mg MDA $/ \mathrm{kg}$ flesh are generally regarded as the limit of acceptability for fish stored (Kadir Topuz et al. 2015). In case of fish oils, Okada et al. (Okada \& Morrisset 2007) obtained TBARS values around $10 \mathrm{mg}$ MDA/kg for freshly extracted sardine oil. The refined sardine oil used in this work presents a value of $46 \mathrm{mg}$ of 
$\mathrm{MDA} / \mathrm{kg}$ of oil, however, as it was refined and stored at $-18^{\circ} \mathrm{C}$ up to experiments, it is difficult compared to previous values. It can be also found a document by FAO that establishes for fresh fish oil a TBARS value of $50 \mathrm{mg}$ of $\mathrm{MDA} / \mathrm{kg}$ fish oil, although it is referred to fishes feed (Masson S 1994). The Rancimat method was also used to estimate the oxidative stability of refined sardine oil. The time until secondary reaction products are detected is called induction time. The induction period (h) by Rancimat, determined at $70^{\circ} \mathrm{C}$ and $20 \mathrm{~L} / \mathrm{h}$ of air flow rate was 7.2 hours, which is a similar oxidative stability index (OSI) compared with those obtained by Noriega et al. (Noriega-Rodríguez et al. 2009) for crude and deodorized sardine oil (10.4 and $17.7 \mathrm{~h}$, respectively) using Rancimat at $60^{\circ} \mathrm{C}$ and $7 \mathrm{~L} / \mathrm{h}$ of air flow rate.

\subsection{Oxidation kinetics of sardine oil at different temperatures}

\section{Kinetic aspects of oxidation products}

Peroxide values at the three temperatures studied in this work, 40,65 and $90^{\circ} \mathrm{C}$ were determined over time during $10 \mathrm{~h}$ (Fig.6.1A). At any incubation time, peroxide value increased with incubation temperature. For all temperatures assayed in this work, no induction period was observed, probably due to the high amount of n-3 PUFA of sardine oil (40\%) and the initial content of peroxides ( 4.8 meq $\mathrm{O}_{2} / \mathrm{kg}$ oil). In a typical curve of oxidation, the PV increases up to a certain maximal and then it begins to decompose into secondary oxidation products. Fig.6.1A shows that peroxide value continuously increased with time for $10 \mathrm{~h}$. This means that hydroperoxide rate formation is still higher than hydroperoxide rate decomposition in the period of time covered in this work for all the temperatures. The kinetics of lipid oxidation is not an easy task. Reactions are usually described by a pseudo-zero, pseudo-first and pseudo second order reactions. In any case, the order or the reaction rate does not comply with the stoichiometry of the reaction (Kamal-Eldin \& Yanishlieva 2005). Labuza and Bergquist (Labuza \& Bergquist 1983) found that lipid oxidation were half-order with respect to pure lipids in model systems. However, in complex food systems the data sometimes fit zero order as well (Labuza \& Bergquist 1983). 
Table 6.2. Fatty acid composition and chemical characteristics of the refined sardine oil used in this work.

\begin{tabular}{|c|c|c|}
\hline \multirow{18}{*}{ Fatty acid profile (wt\%) } & C14:0 & $6.3 \pm 0.2$ \\
\hline & $\mathrm{C} 16: 0$ & $17.3 \pm 0.3$ \\
\hline & C16:1n-7 & $8.4 \pm 0.1$ \\
\hline & C16:2n-4 & $1.1 \pm 0.0$ \\
\hline & C16:3n-4 & $1.2 \pm 0.0$ \\
\hline & C16:4n-1 & $2.1 \pm 0.0$ \\
\hline & C18:0 & $3.7 \pm 0.0$ \\
\hline & C18:1n-9 & $13.9 \pm 0.1$ \\
\hline & C18:1n-7 & $3.7 \pm 0.0$ \\
\hline & C18:2n-6 & $2.9 \pm 0.0$ \\
\hline & C18:4n-3 & $2.1 \pm 0.0$ \\
\hline & C20:1n-9 & $2.6 \pm 0.0$ \\
\hline & $C 20: 3 n-3$ & $1.1 \pm 0.0$ \\
\hline & $C 20: 5 n-3$ & $15.7 \pm 0.3$ \\
\hline & $C 22: 1 n-11$ & $1.9 \pm 0.0$ \\
\hline & $C 22: 5 n-3$ & $1.9 \pm 0.1$ \\
\hline & $C 22: 6 n-3$ & $8.2 \pm 0.3$ \\
\hline & Others $<1 \%$ & $6 \pm 0.1$ \\
\hline PUFA \% & & $39.6 \pm 0.8$ \\
\hline n3 \% & & $31.2 \pm 0.7$ \\
\hline $\mathrm{EPA}+\mathrm{DHA}$ & & $23.9 \pm 0.6$ \\
\hline Polyene index & & $1.39 \pm 0.01$ \\
\hline Acid value (mg KOH/kg)* & & $0.45 \pm 0.04$ \\
\hline Peroxide value (meq. $\mathrm{O}_{2} / \mathrm{kg}$ ) & & $4.8 \pm 0.6$ \\
\hline Anisidine value & & $19.7 \pm 0.4$ \\
\hline TBARS (mg malondialdehido/kg) & & $46 \pm 4$ \\
\hline Induction period (h) by Rancimat & $\left.0^{\circ} \mathrm{C}\right)$ & $7.2 \pm 0.7$ \\
\hline
\end{tabular}

*According to AOCS, Ca 5a-40. 


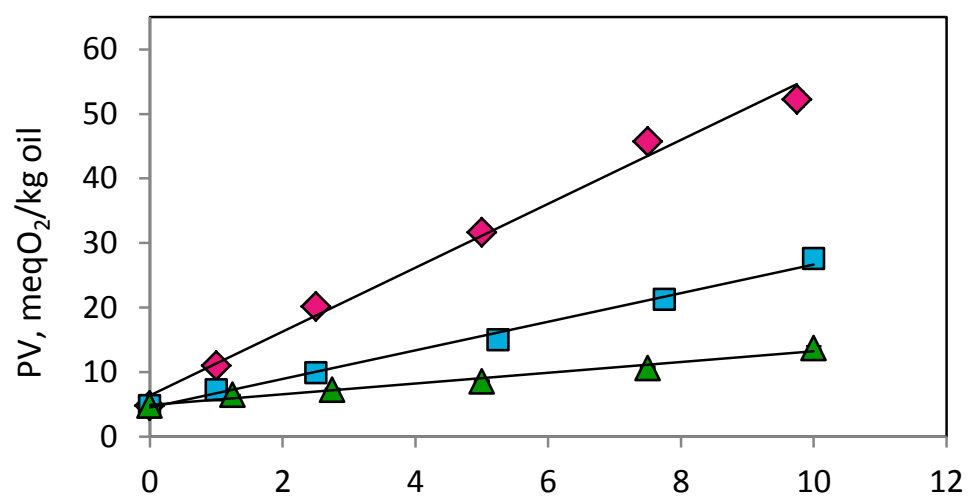

A)

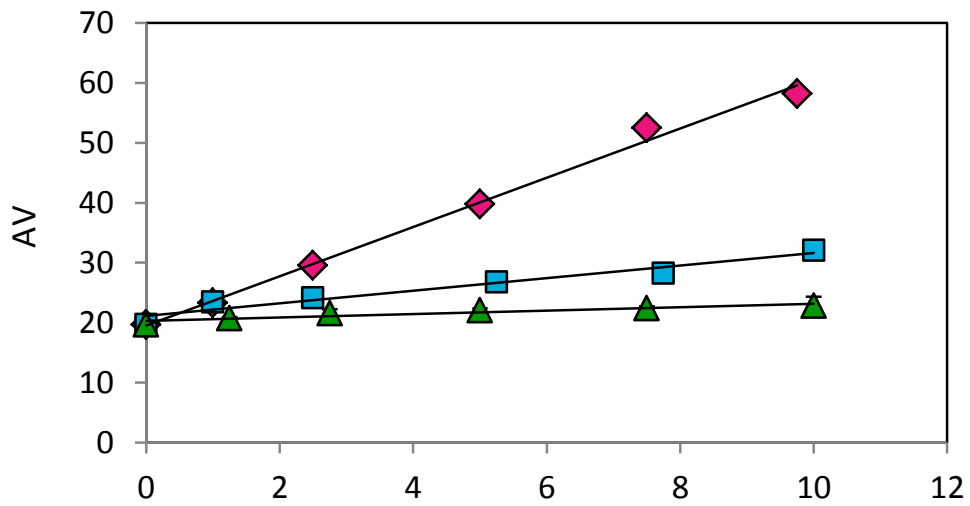

B)

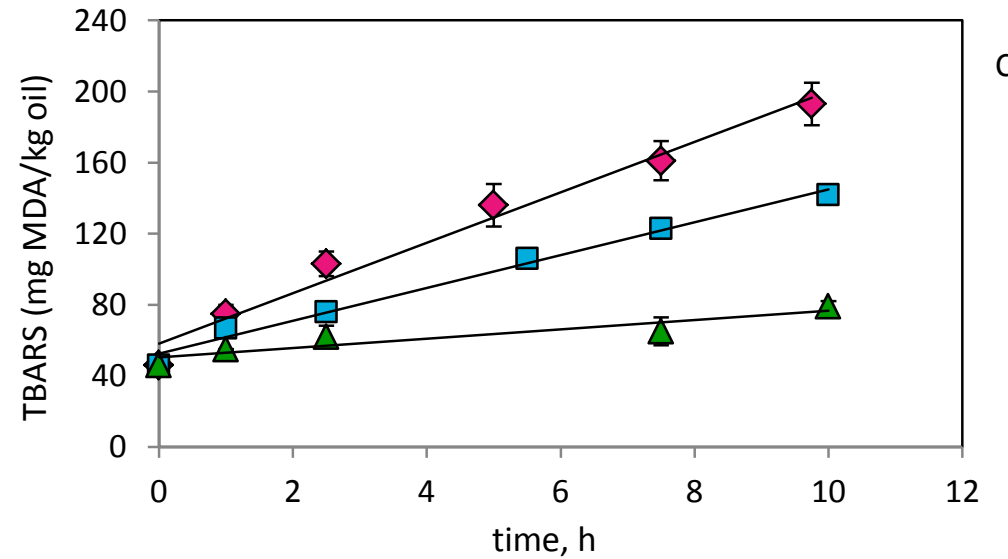

Fig.6.1. $P V(A), A V(B)$ and TBARS $(C)$ of sardine oil during incubation time at $40^{\circ} \mathrm{C}(\mathrm{A})$, $65^{\circ} \mathrm{C}(\square)$ and $90^{\circ} \mathrm{C}(\Delta)$. 
In this work, formation of primary oxidation products follows a pseudo-zero order kinetic model being independent of the oxidizing substrate

$\mathrm{PV}=\mathrm{PV} \mathrm{o}_{\mathrm{o}}+\mathrm{k} \cdot \mathrm{t}$

where $\mathrm{PV}_{\mathrm{o}}$ denotes the initial concentration of peroxide value and $\mathrm{k}$ is the reaction rate constant for a zero order reaction $\left(\right.$ meq $\mathrm{O}_{2} \cdot \mathrm{kg} \mathrm{oil}^{-1} \cdot \mathrm{h}^{-1}$ ). Table 6.3 lists the reaction rate constant for the three temperatures assayed in this work, together with the quality of the fitting. Pseudo-first and pseudo second order kinetics were also tried, but the fitting was worse than for pseudo-zero order reaction. Gomez-Alonso et al. (Gómez-Alonso et al. 2004) also found that kinetics of primary oxidation products of olive oil in the temperature range from 25 to $75^{\circ} \mathrm{C}$ followed a pseudo-zero order kinetics.

Regarding the oxidation kinetics of secondary oxidation products, Fig.6.1B and 6.1C show the variation of the $\mathrm{AV}$ and the TBARS, respectively, over time at the three temperatures studied in this work. It can be observed that, both AV and TBARS, continuously increased with time and temperature yielding a high amount of secondary oxidation products in the first $10 \mathrm{~h}$ of incubation time, mainly at the highest temperatures, 90 and $65^{\circ} \mathrm{C}$. At $40^{\circ} \mathrm{C}$ the oxidation kinetics were much slower and the oxidation values did not increase in a great extent. Comparing Fig.6.1A, 6.1B and 6.1C it can be observed that generation of secondary oxidation products is taking place simultaneously with hydroperoxides formation. However, in the temperature range covered in this work for $10 \mathrm{~h}$ of incubation time, PV formation is still higher than its decomposition into secondary oxidation products. Similar behavior has been also observed for vegetables oils, such as olive and rapeseed oil, in which it was observed that secondary oxidation products begin to be formed nearly at the same time as hydroperoxides generation (Guillen \& Cabo 2002). Gomez-Alonso et al. (Gómez-Alonso et al. 2004) proposed a pseudo-first order reaction for the formation of secondary oxidation products, AV, in olive oil. However, similar to PV formation, the best fitting for AV and TBARS was obtained for a pseudo-zero order reaction. Reaction rate constants for this model are collected in Table 6.3 together with the quality of the fitting. The kinetics of formation of secondary oxidation products of PUFA has not yet been well investigated (Kamal-Eldin \& Yanishlieva 2005). From Fig.6.1B and 6.1C and the values of the kinetic constant, it can be observed that TBARS formation is faster than AV. Some 
authors support the theory that TBARS are formed in considerable amounts mainly from PUFA containing three or more double bonds (Hoyland \& Taylor 1991). Therefore the faster increase of TBARS regarding AV could be due to the high PUFA content of the refined sardine oil.
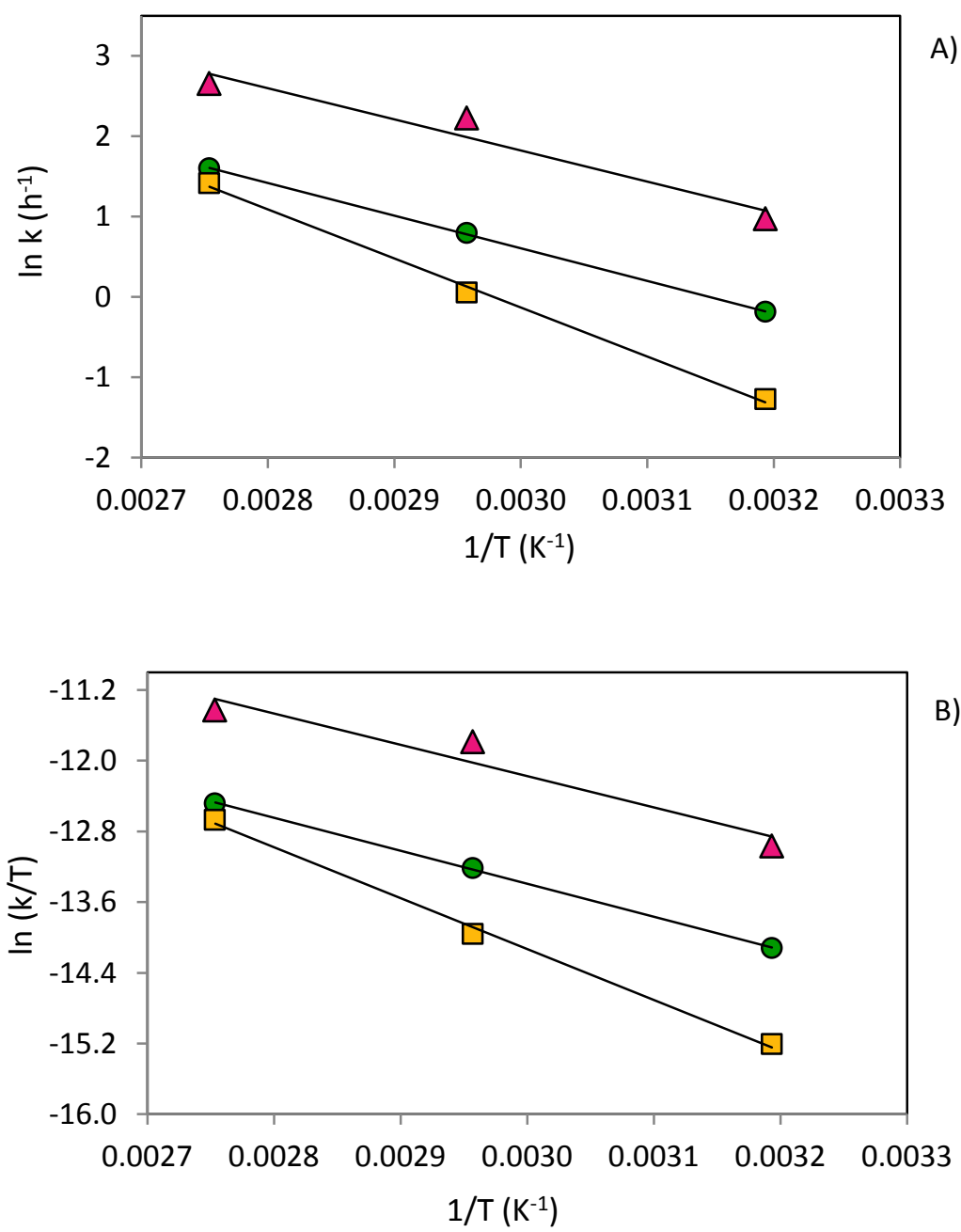

Fig.6.2. Arrhenius plot (A) and Eyring plot (B) of the oxidation of sardine oil, PV $(\bullet), A V(\square)$, $\operatorname{TBARS}(\Delta)$ 
Table 6.3. Kinetic rate constant $(k)$, activation energy $\left(E_{a}\right)$, enthalpy $\left(\Delta H^{\neq}\right)$, entropy $\left(\Delta S^{\neq}\right)$and Gibb's free energy $\left(\Delta G^{\ddagger}\right)$ of activation for primary and secondary oxidation of the sardine oil at different temperatures

\begin{tabular}{|c|c|c|c|c|c|c|c|c|c|c|}
\hline & & \multicolumn{3}{|c|}{ PV } & \multicolumn{3}{|c|}{ AV } & \multicolumn{3}{|c|}{ TBARS } \\
\hline & & k & $\mathbf{R}^{2}$ & $\begin{array}{c}\Delta \mathrm{G}^{\neq} \\
\left(\mathrm{kJ} \cdot \mathrm{mol}^{-1}\right)\end{array}$ & k & $\mathbf{R}^{2}$ & $\begin{array}{c}\Delta G^{f} \\
\left(k J \cdot \mathrm{mol}^{-1}\right)\end{array}$ & k & $\mathbf{R}^{2}$ & $\begin{array}{c}\Delta G^{F} \\
\left(k J \cdot \mathrm{mol}^{-1}\right)\end{array}$ \\
\hline & 40 & $0.831 \pm 0.06$ & 0.9797 & $98.5 \pm 4$ & $0.280 \pm 0.05$ & 0.8690 & $101.4 \pm 1$ & $2.631 \pm 0.60$ & 0.8669 & $95.2 \pm 19$ \\
\hline \multirow[t]{2}{*}{$\mathrm{T},{ }^{\circ} \mathrm{C}$} & 65 & $2.217 \pm 0.09$ & 0.9933 & $103.6 \pm 4$ & $1.053 \pm 0.13$ & 0.9444 & $105.2 \pm 1$ & $9.245 \pm 0.53$ & 0.9869 & $100.2 \pm 20$ \\
\hline & 90 & $4.951 \pm 0.23$ & 0.9912 & $108.8 \pm 4$ & $4.109 \pm 0.15$ & 0.9944 & $109.1 \pm 1$ & $14.202 \pm 1.04$ & 0.9791 & $105.3 \pm 21$ \\
\hline \multicolumn{2}{|c|}{$\mathrm{E}_{\mathrm{a}}\left(\mathrm{kJ} \cdot \mathrm{mol}^{-1}\right)$} & \multicolumn{3}{|c|}{$37.1 \pm 2$} & \multicolumn{3}{|c|}{$55.9 \pm 0.5$} & \multicolumn{3}{|c|}{$35 \pm 10$} \\
\hline \multicolumn{2}{|c|}{$\Delta \mathrm{H}^{\neq}\left(\mathrm{kJ} \cdot \mathrm{mol}^{-1}\right)$} & \multicolumn{3}{|c|}{$34 \pm 2$} & \multicolumn{3}{|c|}{$53 \pm 1$} & \multicolumn{3}{|c|}{$32 \pm 10$} \\
\hline \multicolumn{2}{|c|}{$\Delta \mathrm{S}^{\neq}\left(\mathrm{J} \cdot \mathrm{mol}^{-1} \cdot \mathrm{K}^{-1}\right)$} & \multicolumn{3}{|c|}{$-206 \pm 6$} & \multicolumn{3}{|c|}{$-155 \pm 2$} & \multicolumn{3}{|c|}{$-202 \pm 30$} \\
\hline
\end{tabular}


Regarding the polyene index, it was determined at the end of the kinetics at the three temperatures. The EPA and DHA content and palmitic acid (C16:0) did not change significantly compared to the initial composition of sardine oil. Small variations were observed but those were within the variability associated to the chromatographic method.

This result agrees with the work of Sullivan et al. (Sullivan et al. 2015) in the study of oxidation rates of triacylglycerols and ethyl estersof fish oil. These authors suggested that GC-FID might not be sensitive enough to detect changes in EPA and DHA concentration that could take place as a consequence of oxidation.

\section{Thermodynamic aspects of sardine oil oxidation}

The dependence of the kinetic rate constant on temperature is usually represented by an Arrhenius type relationship:

$\operatorname{lnk}=\ln k_{\mathrm{o}}-\frac{\mathrm{E}_{\mathrm{a}}}{\mathrm{RT}}$

where $k$ is the reaction rate constant, $k_{o}$ is the pre-exponential factor, $E_{a}$ the activation energy and $\mathrm{R}$ the universal gas constant. Fig.6.2A shows the Arrhenius plot for the kinetics of the primary and secondary oxidation products of sardine oil. The activation energy for each oxidation product formation is reported in Table 6.3. For peroxide formation, an activation energy of $37.1 \mathrm{~kJ} / \mathrm{mol}$ was obtained. This value was compared to other studies found in the literature, which are summarized in Table 6.4. Yin et al. (Yin \& Sathivel 2010) obtained a similar value for the $E_{a}(30.9 \mathrm{~kJ} / \mathrm{mol})$ in the study of peroxides formation in the unrefined menhaden oil in the temperature range from 45 to $85{ }^{\circ} \mathrm{C}$ and Sathivel et al. (Sathivel et al. 2008) also reported similar $E_{a}(33.2 \mathrm{~kJ} / \mathrm{mol})$ for the hydroperoxides formation in the unrefined pollock oil. The lower $E_{a}$ for fish oils is expected because less energy should be required to initiate oxidation due to the higher $n-3$ PUFA content compared to vegetable oils. Furthermore, vegetable oils have high content of natural antioxidants, such as tocopherols (Lee et al. 2007). Therefore as it can be seen in Table 6.4, the autoxidation of vegetable oils were more temperature-dependent than fish oils autooxidation.In case of omega-3 concentrated fish oils such as NutraSea, the high $E_{a}$ is 
due to the amount of antioxidant added, $E_{a}$ increasing with increased concentration of antioxidant (Sullivan et al. 2011).

The dependence on temperature of the reaction rate constants of the secondary oxidation products, AV and TBARS, also followed an Arrhenius type relationship with an activation energy of $55.9 \mathrm{~kJ} / \mathrm{mol}$ for $\mathrm{AV}$ and $35 \mathrm{~kJ} / \mathrm{mol}$ for TBARS. The higher activation energy for the non-volatile secondary oxidation products (mainly 2-alkenals and 2,4-dienals) demonstrates that AV formation is more temperature sensitive than hydroperoxides and TBARS formation. For these oxidation compounds, PV and TBARS, similar values of $E_{a}$ were obtained. This similarity could be related to the mechanism proposed by some authors for malonaldehyde formation from peroxides which are produced from fatty acids containing three or more double bonds (Hoyland \& Taylor 1991).

Table 6.4. $E_{a}$ from the Arrhenius equation for the formation of hydroperoxides in different kind of oils found in literature.

\begin{tabular}{|c|c|c|c|}
\hline Type of oil & $\begin{array}{l}\text { T range } \\
\left({ }^{\circ} \mathrm{C}\right)\end{array}$ & $\begin{array}{c}\mathrm{E}_{\mathrm{a}} \\
\mathrm{kJ} / \mathrm{mol}\end{array}$ & Reference \\
\hline Refined sardine oil & $40-90$ & 37.1 & This work \\
\hline Unrefined menhaden oil & $45-85$ & 30.9 & (Yin \& Sathivel 2010) \\
\hline Unrefined pollock oil & $24-90$ & 33.2 & (Sathivel et al. 2008) \\
\hline $\begin{array}{l}\text { NutraSea }(18 \% \text { EPA + 12\% DHA })^{1} \\
\text { NutraSea HP }(30 \% \text { EPA + 10\% DHA })^{1}\end{array}$ & $20-60$ & $\begin{array}{l}76 \\
55\end{array}$ & (Sullivan et al. 2011) \\
\hline Virgin olive oil ${ }^{2}$ & $25-75$ & 32.1 & $\begin{array}{c}\text { (Gómez-Alonso et al. } \\
\text { 2004) }\end{array}$ \\
\hline Refined soybean oil & & 73.6 & \\
\hline Refined sunflower oil & $25-80$ & 79.5 & (Lee et al. 2007) \\
\hline Virgin olive oil & & 52.3 & \\
\hline
\end{tabular}

${ }^{1}$ with antioxidants added

${ }^{2}$ stripped of pro- and antioxidants

Enthalpy $\left(\Delta \mathrm{H}^{\neq}\right)$and entropy $\left(\Delta \mathrm{S}^{\neq}\right)$of activation were also estimated by applying the equation of Eyring (Eq. 3) by plotting $\ln (\mathrm{k} / \mathrm{T})$ vs $1 / \mathrm{T}$ : 
$\ln \frac{k}{T}=\ln \frac{k_{B}}{h}+\frac{\Delta S^{\neq}}{R}-\frac{\Delta H^{\neq}}{R T}$

where $k_{B}$ is the Boltzmann constant $\left(1.38065 \cdot 10^{23} \mathrm{~J} / \mathrm{K}\right)$ and $\mathrm{h}$ is the Planck's constant $\left(6.62608 \cdot 10^{-34} \mathrm{~J} \cdot \mathrm{s}\right)$. Fig.6.2B shows the Eyring plot for kinetics of the primary and secondary oxidation products of sardine oil. From the slope and the intercept, the values of $\Delta \mathrm{H}^{\neq}$and $\Delta \mathrm{S}^{\neq}$have been calculated and presented in Table 6.3. $\Delta \mathrm{H}^{\neq}$is the energy involved in the process during oxidation, the positive value of $\Delta \mathrm{H}^{\neq}$for the primary and secondary oxidation products indicates that the oxidation process is endothermic. According to the transition-state theory, $E_{a}$ is directly proportional to the enthalpy $\left(E_{a}=\Delta H^{\neq}\right)$ (Pazhouhanmehr et al. 2016). The higher value of $\Delta \mathrm{H}^{\neq}$for $\mathrm{AV}$, compared to PV and TBARS indicates lower reactivity. $\Delta S^{\neq}$is related to the degree of disorder of a system, in a spontaneous process the energy of the system tends to decrease, while the total entropy tends to increase (Silva Rodrigues et al. 2017). The negative value for $\Delta S^{\neq}$, in all the oxidation reactions, is a result of association mechanism between reacting species to form the transition state during the reaction. The Gibb's free energy of activation $\left(\Delta \mathrm{G}^{\neq}\right)$was calculated for each temperature:

$\Delta G^{\neq}=\Delta H^{\neq}-T \Delta S^{\neq}$

Negative values of $\Delta G^{\neq}$indicate a spontaneous reaction, while positive values indicate nonspontaneous reaction. Table 6.3 also reported the $\Delta \mathrm{G}^{\neq}$at the three temperatures for all the oxidation kinetics. The positive values of the Gibbs's free energy of activation also show the non-spontaneity of this process. The increase of $\Delta G^{\neq}$with temperature observed is related to the endothermic oxidation mechanism (Pazhouhanmehr et al. 2016).

\subsection{Oxidation status of sardine oil in contact with different immobilized lipases}

Table 6.5 presents the different PV, AV and TBARS over time for sardine oil in contact with the three immobilized lipases selected in this work, at different incubation temperatures. 
Results will be discussed by comparing the values obtained in the absence of immobilized lipase (section 3.2), at different temperatures and support characteristics.

The initial PV value for sardine oil was $4.8 \mathrm{meq}_{2} / \mathrm{kg}$ and, in the previous oxidation kinetic studies, it was observed that PV increased continuously with time at the three temperatures reaching values of 52,25 and 10 meq $\mathrm{O}_{2} / \mathrm{kg}$ at 90,65 and $40^{\circ} \mathrm{C}$, respectively after $10 \mathrm{~h}$. However, when sardine oil was in contact with the immobilized lipases, PV were much lower than the values corresponding to the normal kinetic oxidation process (Fig.6.1A), being even lower than the initial PV for sardine oil. This decrease in primary oxidation products could be attributed to the adsorption capacity of the different lipases supports. In any case, as it has been explained in the introduction section, this decrease in primary oxidation products is a complex balance between formation, decomposition and adsorption. Additionally, supports could even catalyzed degradation of peroxides.

The adsorption process seems to be favored by an increase of temperature for the three immobilized lipases, since from the values listed in Table 6.5 it can be observed that PV are lower the higher the incubation temperature. Nevertheless different behavior can be observed between the three supports where the lipases are immobilized. At $10 \mathrm{~h}$ of contact time, at the highest temperature, $90^{\circ} \mathrm{C}$, PV of sardine oil in contact with Lipozyme RM, immobilized on a weak base anion exchange resin (Rodrigues \& Fernandez-Lafuente 2010), reached the lowest value, followed by Lipozyme 435, immobilized on a macroporous resin of poly-methylmethacrylate and the highest PV was obtained for Lipozyme TL, $1.9<3.3<4.0$ for each support respectively. The same trend was observed for 65 and $40^{\circ} \mathrm{C}$. However, at $40^{\circ} \mathrm{C}$ the PV of sardine oil in contact with the immobilized lipases was no significantly different than those reported previously for oxidation of sardine oil, even in some cases higher values were obtained, especially in those samples in contact with Lipozyme RM and Lipozyme TL. Therefore, at $40^{\circ} \mathrm{C}$ hydroperoxides formation was probably promoted. Similar results have been reported by Toro et al. (Toro-Vazquez et al. 1991), who indicated that peroxide adsorption and/or decomposition predominated at $50{ }^{\circ} \mathrm{C}$, while peroxide formation predominated at $30^{\circ} \mathrm{C}$, especially at low contact times. These results probe that although an increase in temperature leads to an increase in peroxide formation, adsorption of peroxides is much more effective at higher 
temperatures. This agrees with several works in which high temperature is preferred for bleaching process with other type of adsorbents (García-Moreno et al. 2013; NoriegaRodríguez et al. 2009; Strieder et al. 2017).

Regarding secondary oxidation products, AV and TBARS, sardine oil incubated with the immobilized lipases presented lower values than the corresponding to the normal kinetic oxidation process at the highest temperatures 90 and $65^{\circ} \mathrm{C}$ (see Fig.6.1B and $6.1 \mathrm{C}$ ). Lipozyme RM was the only immobilized lipase that slightly decreased the AV below the initial value of sardine oil, 19.7, down to 17.4. For the other two immobilized lipases, AV continuously increased, although much less than in the control sardine oil in the absence of immobilized lipases. Similar results have been found in the literature. García-Moreno et al. (García-Moreno et al. 2013), in their work about bleaching of sardine oil,found a

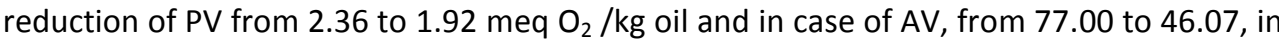
$60 \mathrm{~min}$ at $90^{\circ} \mathrm{C}$, with $3 \%$ of acid-activated clay.

In the case of TBARS, at the highest temperatures, 90 and $65^{\circ} \mathrm{C}$, much lower values were obtained when comparing with the values reported in the absence of immobilized lipases. Similar to PV, oil samples in contact with Lipozyme RM at $90{ }^{\circ} \mathrm{C}$ and $65{ }^{\circ} \mathrm{C}$, reduced their TBARS content below the initial value for sardine oil, from $46 \mathrm{mg} \mathrm{MDA} / \mathrm{kg}$ of oil to 15 $\mathrm{mg} \mathrm{MDA} / \mathrm{kg}$ of oil, almost a $70 \%$ less. On the contrary, at $40{ }^{\circ} \mathrm{C}$, values of the same order or even higher were obtained, in case of samples in contact with Lipozyme TL, than for the control oil in the absence of immobilized lipases.

Based on the oxidation status of sardine oil in contact with the immobilized lipases, it could be concluded that lipase supports have adsorption capacity for both primary and secondary oxidation products at high temperatures $65-90{ }^{\circ} \mathrm{C}$. For all the oxidation products, adsorption capacity follows the order Lipozyme RM > Lipozyme 435 > Lipozyme TL. These differences could be attributed to the different lipase supports (Table 6.1). 
Table 6.5. PV for treated samples in contact with Lipozyme 435, Lipozyme RM and Lipozyme TL at the different times at the three incubation temperatures, 40,65 and $90^{\circ} \mathrm{C}$.

\begin{tabular}{|c|c|c|c|c|c|c|c|}
\hline \multirow{2}{*}{$\begin{array}{l}\text { Oxida- } \\
\text { tion } \\
\text { Index }\end{array}$} & \multirow{2}{*}{$\begin{array}{c}\text { Lipa } \\
\text {-se }\end{array}$} & \multirow{2}{*}{$\begin{array}{c}\mathrm{T} \\
(\mathrm{o})\end{array}$} & \multicolumn{5}{|c|}{ Contact time (h) } \\
\hline & & & 1 & 2.5 & 5 & 7.5 & 10 \\
\hline \multirow{9}{*}{$\begin{array}{c}\mathrm{PV} \\
\text { (meq } \\
\mathrm{O}_{2} / \\
\mathrm{kg} \text { of } \\
\text { oil) }\end{array}$} & \multirow{3}{*}{$\begin{array}{l}\text { Lip. } \\
435\end{array}$} & 90 & $4.3 \pm 0.4^{a}$ & $3.8 \pm 0.1^{\mathrm{ab}}$ & $3.1 \pm 0.1^{\mathrm{ab}}$ & $3.1 \pm 0.1^{\mathrm{ab}}$ & $3.3 \pm 0.3^{b}$ \\
\hline & & 65 & $4.6 \pm 0.1^{a}$ & $4.6 \pm 0.1^{b c}$ & $4.3 \pm 0.5^{c d}$ & $3.9 \pm 0.1^{b}$ & $3.9 \pm 0.1^{c}$ \\
\hline & & 40 & $4.6 \pm 0.2^{a}$ & $5.2 \pm 0.0^{c d}$ & $6.5 \pm 0.0^{e}$ & $5.3 \pm 0.5^{c}$ & $5.0 \pm 0.1^{d}$ \\
\hline & \multirow{3}{*}{$\begin{array}{l}\text { Lip. } \\
\text { RM }\end{array}$} & 90 & $4.8 \pm 0.3^{a}$ & $3.6 \pm 0.3^{\mathrm{a}}$ & $2.7 \pm 0.2^{\mathrm{a}}$ & $2.2 \pm 0.1^{a}$ & $1.9 \pm 0.1^{a}$ \\
\hline & & 65 & $4.4 \pm 0.3^{a}$ & $3.6 \pm 0.2^{a}$ & $2.9 \pm 0.1^{\mathrm{ab}}$ & $2.5 \pm 0.2^{\mathrm{a}}$ & $2.4 \pm 0.1^{\mathrm{a}}$ \\
\hline & & 40 & $4.6 \pm 0.1^{a}$ & $5.4 \pm 0.2^{c d}$ & $8.3 \pm 0.3^{f}$ & $7.4 \pm 1.0^{d}$ & $5.1 \pm 0.4^{d}$ \\
\hline & \multirow{3}{*}{$\begin{array}{c}\text { Lip. } \\
\text { TL }\end{array}$} & 90 & $5.0 \pm 0.0^{a}$ & $5.3 \pm 0.2^{c d}$ & $3.6 \pm 0.0^{b c}$ & $2.9 \pm 0.1^{\mathrm{ab}}$ & $4.0 \pm 0.3^{c}$ \\
\hline & & 65 & $5.4 \pm 0.2^{\mathrm{a}}$ & $5.6 \pm 0.2^{d}$ & $4.6 \pm 0.2^{d}$ & $3.9 \pm 0.1^{b c}$ & $3.5 \pm 0.2^{b c}$ \\
\hline & & 40 & $5.2 \pm 0.0^{\mathrm{a}}$ & $11.9 \pm 0.7^{\mathrm{e}}$ & $15.0 \pm 0.1^{g}$ & $11.9 \pm 0.5^{\mathrm{e}}$ & $9.1 \pm 0.0^{\mathrm{e}}$ \\
\hline \multirow{9}{*}{ AV } & \multirow{3}{*}{$\begin{array}{l}\text { Lip. } \\
435\end{array}$} & 90 & $22.0 \pm 0.0^{\mathrm{de}}$ & $26.0 \pm 0.0^{\mathrm{e}}$ & $25.1 \pm 0.4^{d}$ & $28.2 \pm 0.2^{\mathrm{e}}$ & $26.5 \pm 0.5^{c}$ \\
\hline & & 65 & $22.1 \pm 0.5^{\mathrm{de}}$ & $22.3 \pm 1.2^{\mathrm{cd}}$ & $22.2 \pm 0.2^{c}$ & $23.4 \pm 0.6^{b c}$ & $25.9 \pm 0.8^{c}$ \\
\hline & & 40 & $21.0 \pm 0.4^{\mathrm{cd}}$ & $21.5 \pm 0.3^{b c}$ & $21.6 \pm 0.2^{c}$ & $21.5 \pm 0.7^{b}$ & $22.1 \pm 0.9^{b}$ \\
\hline & \multirow{3}{*}{$\begin{array}{l}\text { Lip. } \\
\text { RM }\end{array}$} & 90 & $19.5 \pm 0.4^{\mathrm{ab}}$ & $19.2 \pm 0.7^{\mathrm{a}}$ & $19 \pm 0.3^{\mathrm{ab}}$ & $17.9 \pm 0.7^{a}$ & $17.4 \pm 0.2^{a}$ \\
\hline & & 65 & $20.0 \pm 0.3^{b c}$ & $19.8 \pm 0.3^{\mathrm{ab}}$ & $18.2 \pm 0.1^{a}$ & $17.7 \pm 0.7^{\mathrm{a}}$ & $18.9 \pm 0.5^{a}$ \\
\hline & & 40 & $18.8 \pm 0.1^{\mathrm{a}}$ & $19.4 \pm 0.2^{\mathrm{a}}$ & $19.7 \pm 0.1^{b}$ & $18.2 \pm 1.5^{\mathrm{a}}$ & $19.2 \pm 0.0^{\mathrm{a}}$ \\
\hline & \multirow{3}{*}{$\begin{array}{c}\text { Lip. } \\
\text { TL }\end{array}$} & 90 & $22.8 \pm 0.1^{\mathrm{e}}$ & $24.1 \pm 0.1^{\text {de }}$ & $24.8 \pm 0.1^{d}$ & $26.8 \pm 0.7^{\mathrm{de}}$ & $33.1 \pm 1.2^{d}$ \\
\hline & & 65 & $21.4 \pm 0.3^{d}$ & $21.7 \pm 0.2^{b c}$ & $24.8 \pm 0.5^{d}$ & $24.5 \pm 0.6^{\mathrm{cd}}$ & $25.1 \pm 0.5^{c}$ \\
\hline & & 40 & $21.4 \pm 0.5^{d}$ & $22.0 \pm 0.3^{c}$ & $23.1 \pm 0.8^{c}$ & $22.3 \pm 0.5^{b c}$ & $22.4 \pm 0.2^{b}$ \\
\hline \multirow{9}{*}{$\begin{array}{l}\text { TBARS } \\
\text { (mg } \\
\text { MDA/ } \\
\text { kg of } \\
\text { oil) }\end{array}$} & \multirow{3}{*}{$\begin{array}{l}\text { Lip. } \\
435\end{array}$} & 90 & $60 \pm 4^{c d}$ & $58 \pm 4^{c d}$ & $37 \pm 3^{a b}$ & $34 \pm 6^{b}$ & $42 \pm 2^{b}$ \\
\hline & & 65 & $55 \pm 4^{\mathrm{abcd}}$ & $48 \pm 4^{\mathrm{abc}}$ & $43 \pm 2^{b c}$ & $49 \pm 5^{c}$ & $54 \pm 4^{c}$ \\
\hline & & 40 & $57 \pm 3^{b c d}$ & $56 \pm 3^{b c d}$ & $54 \pm 0^{c}$ & $64 \pm 0^{d}$ & $70 \pm 2^{d}$ \\
\hline & \multirow{3}{*}{$\begin{array}{l}\text { Lip. } \\
\text { RM }\end{array}$} & 90 & $42 \pm 4^{a}$ & $39 \pm 4^{a}$ & $28 \pm 1^{a}$ & $20 \pm 0^{a}$ & $15 \pm 1^{a}$ \\
\hline & & 65 & $51 \pm 4^{a b c}$ & $45 \pm 4^{\mathrm{ab}}$ & $33 \pm 2^{a b}$ & $22 \pm 3^{\mathrm{ab}}$ & $14 \pm 0^{\mathrm{a}}$ \\
\hline & & 40 & $43 \pm 3^{\mathrm{ab}}$ & $59 \pm 3^{c d}$ & $73 \pm 4^{d}$ & $85 \pm 2^{\mathrm{e}}$ & $69 \pm 3^{d}$ \\
\hline & \multirow{3}{*}{$\begin{array}{c}\text { Lip. } \\
\text { TL }\end{array}$} & 90 & $66 \pm 3^{d}$ & $61 \pm 4^{d}$ & $30 \pm 1^{a}$ & $24 \pm 1^{a b}$ & $22 \pm 2^{a}$ \\
\hline & & 65 & $64 \pm 3^{c d}$ & $61 \pm 1^{d}$ & $54 \pm 2^{c}$ & $50 \pm 4^{c d}$ & $37 \pm 5^{b}$ \\
\hline & & 40 & $52 \pm 9^{\mathrm{abcd}}$ & $110 \pm 11^{\mathrm{e}}$ & $143 \pm 7^{e}$ & $120 \pm 5^{f}$ & $105 \pm 2^{\mathrm{e}}$ \\
\hline
\end{tabular}

Values with different letters in each column are significantly different when applying the Tukey's honestly significant difference (HSD) method at $p$-value $\leq 0.05$. 
Lipase support for Lipozyme RM is a weak-base anion exchange resin, having good capability for adsorption process. Besides that, its hydrophilic nature could result in a higher affinity for oxidation products. On the contrary, Lipozyme 435 presents a hydrophobic support (Chen et al. 2008). The secondary oxidation products, as well as hydroperoxides, are considered polar molecules. It is well known that the higher the degree of oxidation of an oil, the more polar the oxidation products usually are (KamalEldin \& Yanishlieva 2005). This could explain the difference observed among both immobilized lipases. In case of Lipozyme TL, although it is immobilized on silica gel, a hydrophilic material, its particle size range is bigger $(250-1000 \mu \mathrm{m})$ and also the true density reported by Zhang et al. (Zhang 2007$)$ is high $\left(1830 \mathrm{~kg} / \mathrm{m}^{3}\right)$. Therefore, its surface area is considerably lower. Particle size is a major physical parameter affecting bleaching performance since adsorption is a surface phenomenon ( $O^{\prime}$ Brien 2004). In Table 6.1 it can be seen than the average values of Lipozyme RM particle size are smaller $(200 \sim 600 \mu \mathrm{m})$ than for the others two immobilized lipases $(300-1000 \mu \mathrm{m})$. When using adsorbents of smaller size, the adsorption increased due to a higher frequency of collisions between adsorbate and adsorbent.In any case, it must be highlighted that the average particle of these immobilized lipases is much higher than the particle size for commercial absorbents such as Tonsil bleaching earths which range from 25 to $150 \mu \mathrm{m}$ (Strieder et al. 2017). Furthermore, the contact time in this work is well above the usual time required in a typical bleaching process (usually $20 \mathrm{~min}$ ) (Monte et al. 2015; Strieder et al. 2017), but in this work the focus is different, since the lipases will be used as biocatalysts with adsorption capacity of oxidation products generated during reaction due to the effect of temperature.

The oxidation status for sardine oil in contact with the immobilized lipases $\left(C_{\text {immob-lipase }}\right)$ has been compared with the kinetic data for sardine oil previously determined in section 3.2 at the same temperature and incubation times $\left(C_{\text {control }}\right), C_{\text {immob-lipase }} / C_{\text {control, for each }}$ oxidation product. The value of PV, AV and TBARS change over time as a result of formation, decomposition and adsorption of oxidation products, therefore the comparison of the oxidation status was done at the different incubation times and it was not considered a constant initial concentration (Gomes de Oliveira \& Marques Porto 2005). Figures $6.3 \mathrm{~A}, 6.3 \mathrm{~B}, 6.4 \mathrm{~A}, 6.4 \mathrm{~B}, 6.5 \mathrm{~A}$ and $6.5 \mathrm{~B}$ show the ratio $C_{\text {immob-lipase }} / \mathrm{C}_{\text {control }}$ for 
the PV, AV and TBARS at 90 and $65^{\circ} \mathrm{C}$, respectively. The oxidation status of sardine oil in contact with the immobilized lipases at $40^{\circ} \mathrm{C}$ showed no difference with the sardine oil in the absence of immobilized lipases, or even higher oxidation values and results have not been plotted. For the three immobilized lipases tested in this work at 65 and $90{ }^{\circ} \mathrm{C}$, the ratio $\mathrm{C}_{\text {immob-lipase }} / \mathrm{C}_{\text {control }}$ decreased over time. But higher reduction of oxidation products was observed at the highest temperature. Comparing Fig.6.3-6.5 higher reduction of primary oxidation products (PV) was reached than for secondary oxidation products. Among secondary oxidation products, TBARS are reduced in a higher extent than AV. As it has been explained previously, Lipozyme RM, immobilized on an anion exchange resin presents the lower $C_{\text {immob-lipase }} / C_{\text {control }}$ ratio for all the oxidation products. Based on the shape of the Fig.6.3-6.5 the following equation has been used to correlate the data over time:

$\mathrm{C}_{\text {immob-lipase,t }} / \mathrm{C}_{\text {control,t }}=\exp \left(-\mathrm{kt}^{\mathrm{n}}\right)$

where $\mathrm{C}_{\text {immob-lipase, }}$ is the concentration of oxidation compounds in sardine oil in contact with immobilized lipases at a certain contact time, $t, C_{\text {control, } t}$ is the concentration of oxidation compounds in control sardine oil (in the absence of immobilized lipases) at the same incubation time, $\mathrm{t}, \mathrm{n}$ is an adjustment parameter and $\mathrm{k}$ is similar to the adsorption constant. The dependence of $k$ on temperature was represented by an Arrhenius type relationship:

$k=k_{o} \cdot \exp \left(-\frac{E_{a}}{R T}\right)$

where $k_{o}$ is the preexponential factor, $E_{a}$ the activation energy and $R$ the universal gas constant. The equation [5] is based on the modified model of Brimberg proposed by Monte et al. (Monte et al. 2015) in the study of reduction of color and oxidation products of carp oil with blends of bleaching earth and activated carbon. In that work, $\mathrm{C}_{\text {control }}$ was considered to be an initial constant value for each product. However, in this work, $\mathrm{C}_{\text {control,t }}$ was the concentration at the corresponding time when heating the oil in the absence of the immobilized lipases. 

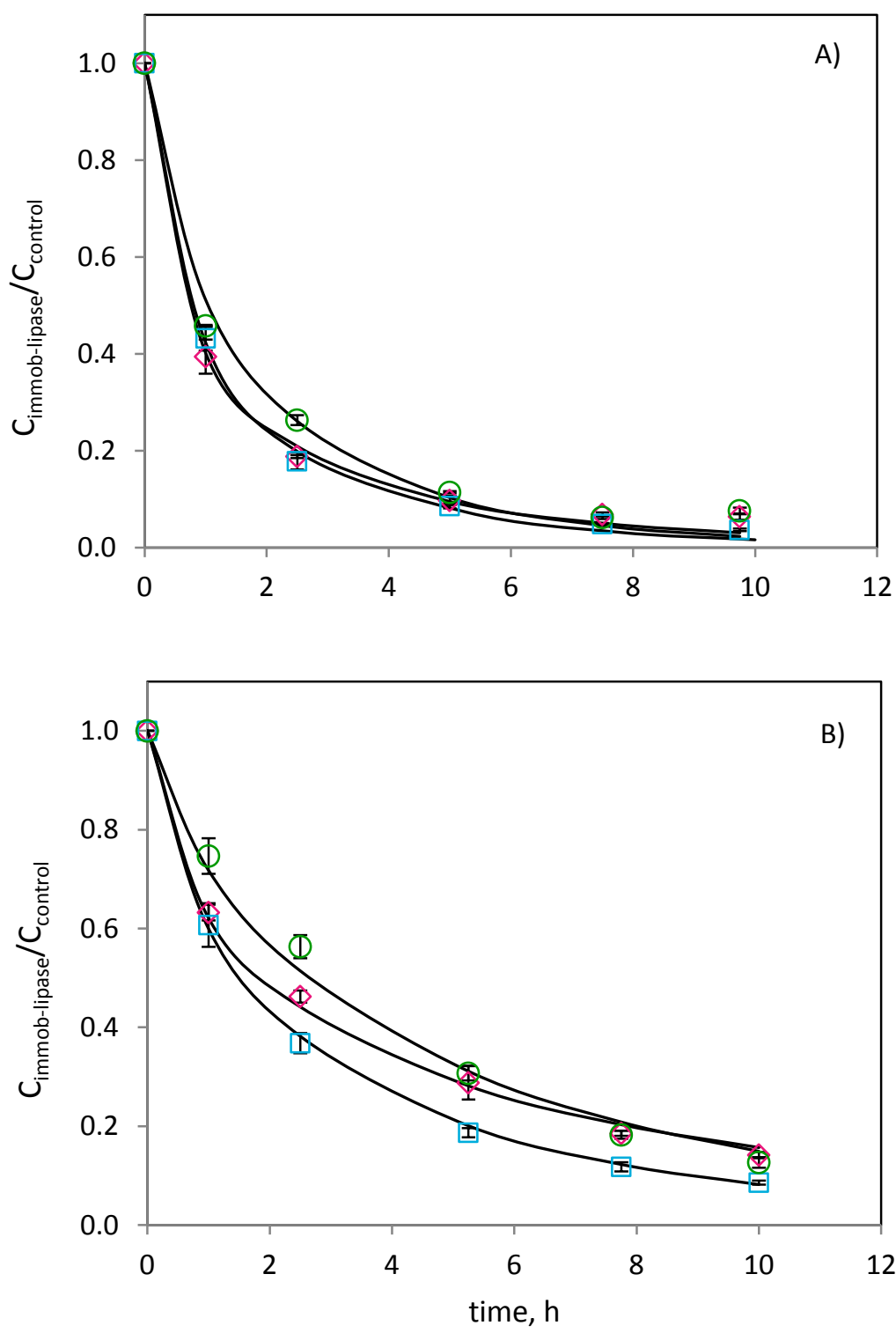

Fig. 6.3. PV ratio between samples in contact with lipases, ( $\diamond)$ Lipozyme 435, ( $\square$ ) Lipozyme $\mathrm{RM},(\mathrm{O})$ Lipozyme $\mathrm{TL}$, and control samples at $90^{\circ} \mathrm{C}(\mathrm{A})$ and $65^{\circ} \mathrm{C}(\mathrm{B})$. Continuous lines represent the values obtained by the equation 5 . 

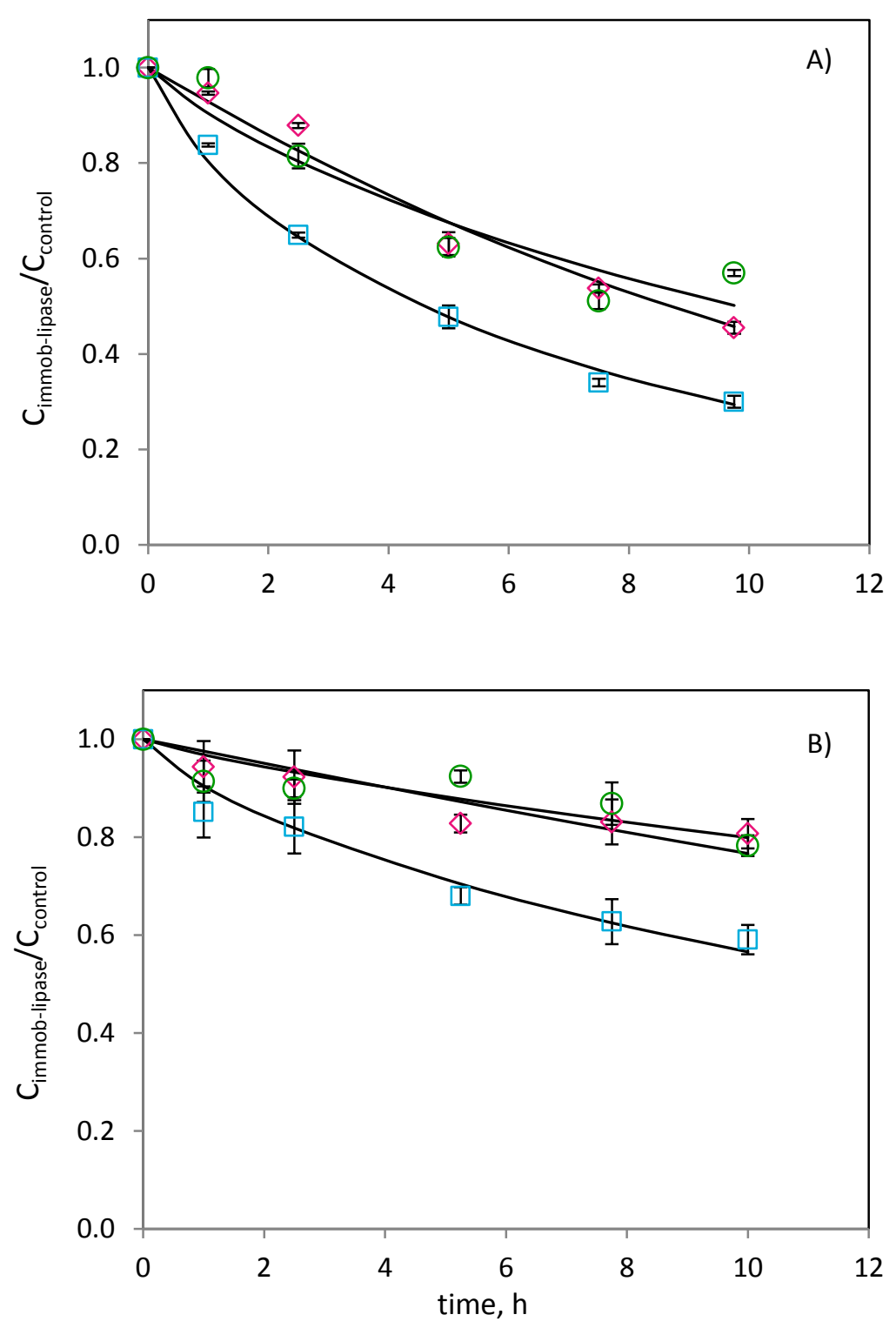

Fig. 6.4. AV ratio between samples in contact with lipases, $(\diamond)$ Lipozyme 435 , ( $\square$ ) Lipozyme $\mathrm{RM},(\mathrm{O})$ Lipozyme $\mathrm{TL}$, and control samples at $90^{\circ} \mathrm{C}(\mathrm{A})$ and $65^{\circ} \mathrm{C}(\mathrm{B})$. Continuous lines represent the values obtained by the equation 5 . 

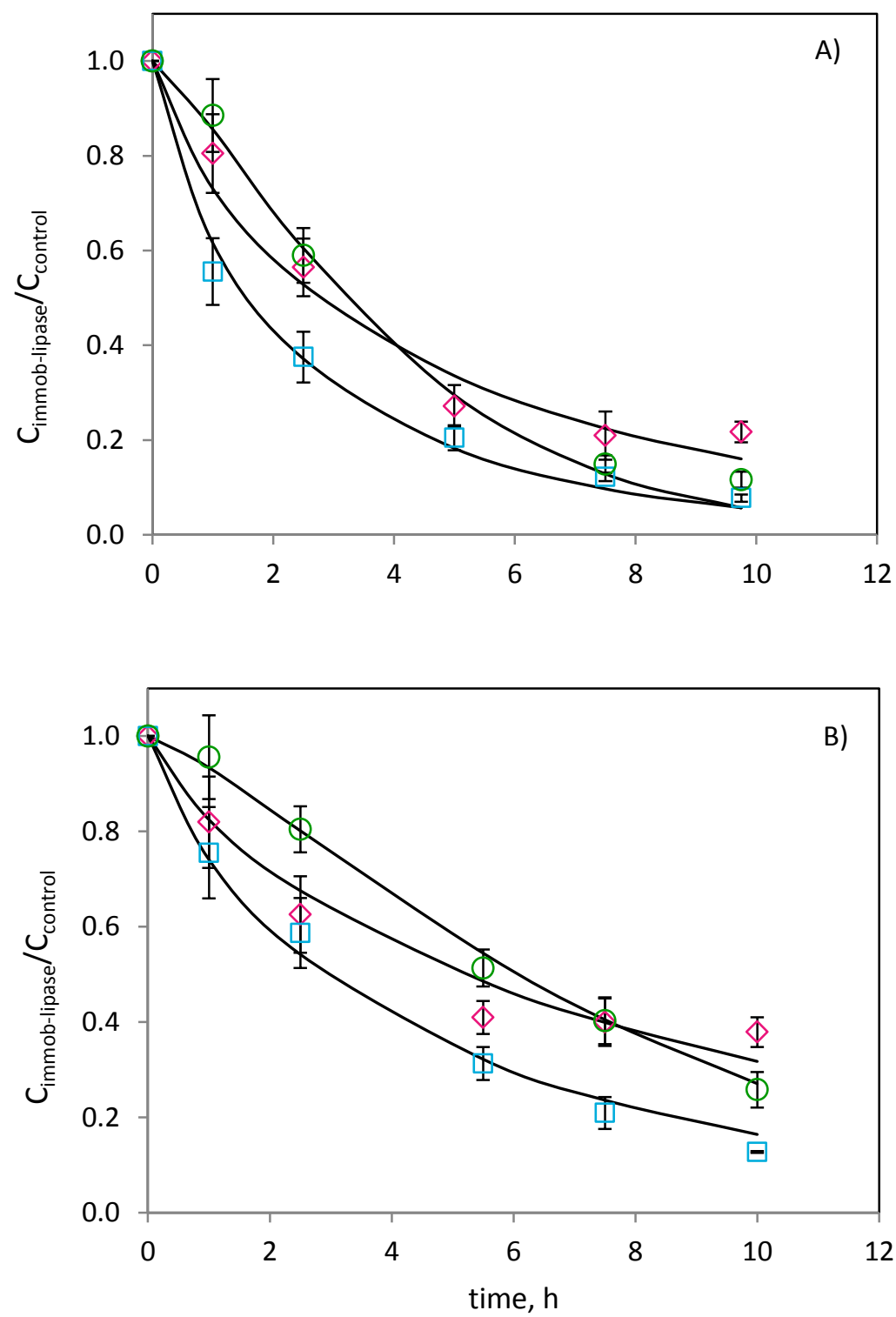

Fig. 6.5. TBARS ratio between samples in contact with lipases, $(\diamond)$ Lipozyme $435,(\square)$ Lipozyme RM, (O) Lipozyme TL, and control samples at $90{ }^{\circ} \mathrm{C}(\mathrm{A})$ and $65^{\circ} \mathrm{C}(\mathrm{B})$. Continuous lines represent the values obtained by the equation 5 . 
Table 6.6. Parameters (equation 5-6) for adsorption of oxidation products quantified by PV, AV and TBARS for the samples in contact with Lipozyme 435, Lipozyme RM and Lipozyme TL.

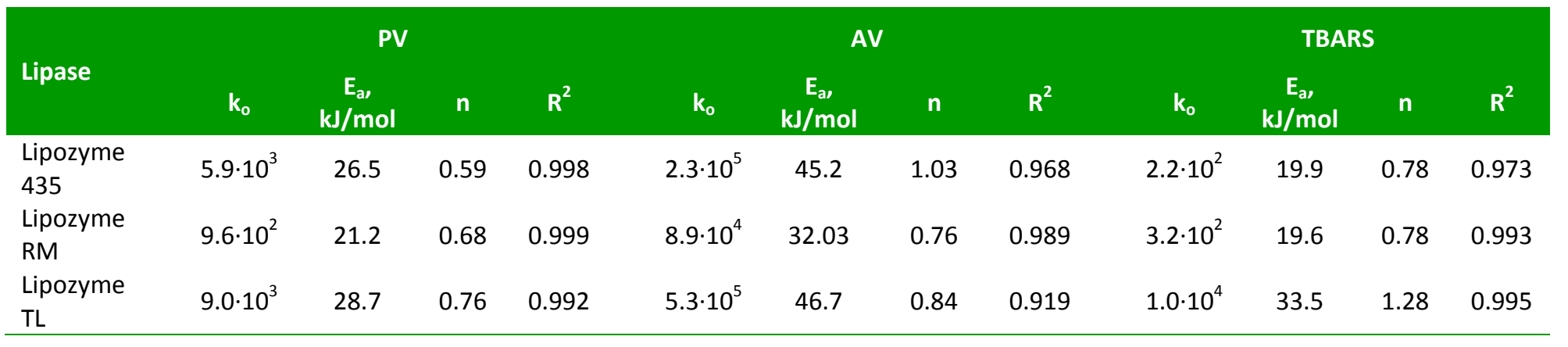


Table 6.6 lists the parameters obtained for the reduction of PV, AV and TBARS for the three immobilized lipases. The lowest $E_{a}$ was obtained for PV and TBARS. For all the oxidation products, the highest $E_{a}$ was found for Lipozyme TL proving that this immobilized lipase is more temperature-dependent on the adsorption of oxidation products. This correlates with the behavior of the lipase supports at different temperatures and their characteristics previously explained. The continuous lines in Fig.6.3-6.5 represent the equation 6 showing good fitting.

On the other hand, the PI was also evaluated in the samples in contact with immobilized lipases after $10 \mathrm{~h}$ at different temperatures but as described in the control sardine oil samples no relevant differences have been observed.

\section{Conclusions}

The oxidation kinetics of sardine oil reveal that PV and TBARS formation is higher than AV, due to the high PUFA content. Commercial immobilized lipases showed good adsorption capacity for sardine oil oxidation products. Lipase supports can both adsorb hydroperoxides and secondary oxidation products at high temperatures, 65 and $90{ }^{\circ} \mathrm{C}$. They seem to be more selective for hydroperoxides and TBARS than for anisidine reacted compounds. However, at $40{ }^{\circ} \mathrm{C}$, oxidation products formation seems to be promoted. Therefore, the use of these immobilized commercial lipases in lipase-catalyzed reactions of fish oils at high temperatures $\left(90^{\circ} \mathrm{C}\right)$, yields high reaction rates but also presents an added benefit by reducing the oxidation values in terms of PV, AV and TBARS. Lipozyme RM results the best immobilized lipase to reduce oxidation indices, quite below to those of the initial fish oil. 
CONCLUSIONS 



\section{CONCLUSIONS}

Although each chapter contains its own conclusions, from the different experimental studies carried out in this $\mathrm{PhD}$ Thesis, the following general conclusions can be established:

- $\quad$ Refined sardine oil contains $30 \%$ of EPA + DHA, being a good source of $n-3$ PUFA. It can be used as a valuable raw material in the production of $n-3$ PUFA concentrates. Among the different lipid derivatives of n-3 PUFA, MAG presents good bioavailability and oxidation stability.

- Lipase-catalyzed glycerolysis reaction is an attractive alternative for polyunsaturated MAG production because the reaction can be carried out under mild conditions, obtaining high reactions yields in short reaction time.

- Knowledge of the phase behavior for systems containing fish oil, glycerol and tert-alcohols as reaction media is important for a correct design of the glycerolysis process since this can influence the reaction pathway as well as the further purification steps. LLE data help to minimize the amount of solvent needed to create a homogeneous reaction system, while too much solvent in the reaction medium leads to decrease in the concentration of substrates at the interface, leading to lower reaction rates.

- Lipozyme 435 from Candida antarctica presents high activity in glycerolysis systems, even in the presence of organic solvents. This lipase is not waterdependent and its use as biocatalyst to mediate glycerolysis reaction results in high MAG yields. However, Lipozyme RM from Rhizomucor miehei has been proved to be a water dependent lipase in glycerolysis systems in tert-alcohols, showing no activity without water addition to the reaction system. Although high content of MAG was reached, up to $70 \mathrm{wt} \%$, the present of water in the system leads to FFA production.

- Although the use of tert-alcohols in glycerolysis reaction leads the highest reaction rates and reaction yields, the cost, toxicity and energy required for solvent removal from the product mixture, are important reasons to avoid its use. The pre-emulsification of substrates, glycerol and oil, improved process 
efficiency giving around 75\% of MAG and DAG in 4 hours. The use of SC- $\mathrm{CO}_{2}$ as reaction medium does not show any kinetic advantages in glycerolysis reaction at the pressure range studied in this work ( $15-25 \mathrm{MPa}$ ). Although $\mathrm{SC}-\mathrm{CO}_{2}$ could be used to fractionate the reaction products.

- Molecular distillation has resulted to be an effective process to separate a mixture of acylglycerols, free fatty acids and glycerol, obtained from the enzymatic glycerolysis of sardine oil. This fractionation technique has been also suitable to concentrate n-3 PUFA in MAG form.

- Commercial immobilized lipases showed a good adsorption capacity of lipid oxidation products. Lipase supports can adsorb mainly hydroperoxides and TBARS at high temperatures reducing the oxidation status even bellow the initial one. 


\section{APPENDIX A}

\section{Characterization of triacylglycerol composition of sardine oil by using chromatographic techniques}

Á. García Solaesa, S.L. Bucio, M.T. Sanz, S. Beltrán and S. Rebolleda (2014).

"Characterization of triacylglycerol composition of fish oils by using chromatographic techniques"

Journal of Oleo Science, 63, (5) 449-460 



\section{Abstract}

! Triacylglycerols (TAG) of refined sardineoil was separated by reverse phase high performance liquid chromatography (RP-HPLC) with a binary solvent gradient of acetone/acetonitrile. Different fractions were observed in the chromatogram and TAG species were tentatively identified by subsequent analysis of the fatty acid (FA) profile in each fraction by capillary Gas Chromatography (GC). Peak identities were assigned on the basis of a multiple linear regression analysis by using factors such as carbon number, number of double bonds, number of monounsaturated fatty acids (MUFA) and number of polyunsaturated fatty acids (PUFA) in the molecule as predictors for TAG retention time. A successful correlation was obtained between retention times and the equivalent carbon number (ECN) of triacylglycerols. Regiospecific analysis of fatty acids in the TAG has been conducted by ethanolysis of the sardine oil by using an immobilized lipase. The subsequent separation of 2-monoacylglycerol (2-MAG) by TLC (thin layer chromatography) analysis showed that ethanolysis system is effective for analysis of FA composition at the 2-position in oils containing PUFA. Principal components analysis (PCA) has been also applied to establish correlations between the different fatty acids in the TAG.

\section{Introduction}

The nutritional benefits attributed to fish oils, rich in omega-3 PUFA, have been the basis for the study of the structural composition of triacylglycerols in fish oil (López-Hernández et al. 2004; Perona \& Ruiz-Gutierrez 1999). Typical fish oil may contain more than 150 different TAG molecule species as a complex mixture. The great variety of fatty acids in fish oil, from myristic acid (14:0) to docosahexaenoic (22:6, n-3) acid, causes enormous difficulties in the approach to the TAG analysis of fish oils.

HPLC using refractive index and evaporative light-scattering detectors (ELSD), has been shown to be effective in the analysis of triacylglycerols. Other methods, such as hightemperature gas chromatography (HT-GC) with flame-ionization detector (FID) or mass spectrometry (MS), have been also reported to be effective in the separation, identification and quantitation of triacylglycerols (Lee \& Hastilow 1999). However, analysis 
of highly unsaturated TAG, such as fish oils, by HT-GC cannot be recommended due to thermal degradation and polymerization of highly unsaturated molecules at high elution temperatures. The analysis of edible oils by their triacylglycerol content is also of great importance as quality control and possible origin determination (Moreda et al. 2003).

In this work, relative responses of ELSD in RP-HPLC have been firstly determined by using model mixtures of standard triacylglycerols. Afterwards, analysis of TAG from sardine oil has been carried out by RP-HPLC technique by using gradients of acetone/acetonitrile mixtures. Fractionation of TAG was performed according to their elution time. To identify the TAG present in the sardine oil samples the concept of equivalent carbon number (ECN) has been used. An approach similar to that proposed by Perona et al. and LópezHernández et al. (López-Hernández et al. 2004; Perona \& Ruiz-Gutierrez 1999) has been followed. This procedure considers the retention time of a certain TAG as a function of its total carbon number (CN), its number of double bonds (DB), and the unsaturated FA (mono and polyunsaturated) present in the TAG molecule of interest.

Ethanolysis of sardine oil with immobilized Candida antarctica lipase has been applied to determine the FA composition at the 2-position in TAG. 2-MAG were detected by TLC and separated by two different chromatographic techniques, normal phase HPLC (NP-HPLC) and TLC. 2-MAG were analyzed by GC.

Furthermore, principal components analysis, PCA, has been performed to confirm the most probable fatty acid association in the different fractions obtained by RP-HPLC.

\section{Experimental section}

\subsection{Materials}

Refined sardine oil was kindly provided by Industrias Afines S.L. The triacylglycerols standards were tricaprin (CCC), trilaurin (LLL), trimyristin (MMM), tripalmitin (PPP), tripalmitolein (PoPoPo), tristearin (SSS), triolein (OOO), trilinolein (LoLoLo), trilinolenin $(\operatorname{LnLnLn)}$, triarachidin (AAA) and tridocosahexaenoin (DhDhDh) of purity greater than $98 \%$. They were obtained from Sigma Aldrich. Standards of fatty acid methyl esters were also 
purchased from Sigma-Aldrich.Ethanolysis of sardine oil was carried out with Lipozyme 435, from Candida antarctica lipase immobilized on a macrosporous resine, which was kindly donated by Novozymes (Bagsvaerd, Denmark). Chemicals used for all chromatographic analysis were HPLC grade from VWR.

\subsection{RP-HPLC analysis}

The HPLC separations were done at $30^{\circ} \mathrm{Con}$ a Lichrospher $100 \mathrm{RP}-18(5 \mu \mathrm{m})$ column $(25 \mathrm{~cm} \times 4 \mathrm{~mm}$ i.d.) in a HPLC with ELSD detector (Agilent Technologies 1200 Series Model, Santa Clara CA, United States). The mobile phase was a mixture of acetone-acetonitrile at a flow-rate of $1 \mathrm{~mL} / \mathrm{min}$. The solvent gradient used is shown in Table A.1. The evaporator temperature was $35^{\circ} \mathrm{C}$, air pressure 3.5 bars and gain 9. Between 5 and $15 \mu \mathrm{L}$ of a standard solution (1-10 mg/mL of each standard TAG) in hexane:acetone (1:1) were injected to calibrate the method. These measurements were performed by triplicate. Sardine oil samples (100 mg/mL of hexane:acetone) injected by RP-HPLC were fractionated according to the times obtained from the TAG profile. Separations were repeated at least six times to collect enough sample for the fatty acid methyl esters (FAMEs) analysis. The fractions were stored in special flasks to evaporate the solvent under vacuum using a rotary evaporator (Heibolph VV2000, Schwabach, Germany) at $40^{\circ} \mathrm{C}$. Then, the samples were transferred to screw-capped tubes to carry out the derivatization.

Table A.1. Solvent gradient used to separate TAG in fish oils (mobile phase content expressed as vol \%)

\begin{tabular}{ccc} 
Time, $\min$ & \% Acetone & \% Acetonitrile \\
\hline 0 & 10 & 90 \\
50 & 60 & 40 \\
60 & 100 & 0 \\
65 & 100 & 0 \\
70 & 10 & 90 \\
\hline
\end{tabular}




\subsection{Ethanolysis of fish oils}

Ethanolysis of TAG with immobilized Candida antarctica lipase has been reported to produce an accumulation of 2-MAG in the early stage of the reaction. Although this lipase is non regiospecific, it behaves as 1,3 specific with a great excess of ethanol (Muñío et al. 2008). Ethanolysis of the sardine oil studied in this work has been conducted as describe by Shimada et al.(Shimada et al. 2003): A mixture of oil/ethanol (1:3, w/w) was shaken at $30^{\circ} \mathrm{C}$ during 4 hours with $4 \%$ immobilized Candida antarctica lipase by weight of oil + ethanol. Fish oil and ethanol are not miscible (Bucio et al. 2013) at the weight ratio proposed by Shimada et al. (Shimada et al. 2003). However, after $4 \mathrm{~h}$ of reaction time, the reaction mixture was a homogeneous phase due to the polarity of MAG and the ethyl esters formed during the reaction.

Quantification of the reaction products (monoacylglycerols, diacylglycerols, triacylglycerols and ethyl esters) after $4 \mathrm{~h}$ of reaction time has been carried out by normal phase HPLC (NP-HPLC). Separations were carried out at room temperature in a Lichrospher Diol column ( $5 \mathrm{~mm}, 4 \mathrm{~mm} \times 250 \mathrm{~mm}$ ) and detection was performed in an ELSD (Agilent Technologies 1200 Series Model, Santa Clara CA, United States) at $35^{\circ} \mathrm{C}$ and $0.35 \mathrm{MPa}$. NP-HPLC method and calibration procedure have been previously described in detail (Rebolleda et al. 2012). Fraction corresponding to 2-MAG was collected in special flasks to evaporate the solvent under vacuum using a rotary evaporator (Heibolph VV2000, Schwabach, Germany) at $40^{\circ} \mathrm{C}$. Then, the samples were transferred to screwcapped tubes to carry out the derivatization for conversion to methyl esters and subsequent fatty acid analysis by GC. 2-MAG fraction was also separated by TLC on silicagel plates (Silica gel $60 \mathrm{~F} 254$, Merck) activated by heating at $105^{\circ} \mathrm{C}$ for $30 \mathrm{~min} .200 \mu \mathrm{L}$ of the reaction mixture were directly spotted on the TLC plate. A mixture of standards was also spotted. The plates were then developed in chloroform/acetone/methanol (95:4.5:0.5, v/v/v). Spots of each lipid were visualized by UV lamp. The fraction corresponding to 2-MAG was scraped off from the plates and methylated according to the method of Wewer et al. (Wewer et al. 2013). 
To check that the MAG fraction does not contain 1(3)-MAG, isomers of the 2-MAG, separation of the different acylglycerols has been also performed by TLC on silica gel plates following a procedure similar to the one reported by Muñio et al. (Muñío et al. 2008). In this case, the plates were impregnated by immersion in a hydroethanolic solution $(50 \%, v / v)$ of boric acid $\left(1.2 \%\right.$ in weight) activated again by heating at $100{ }^{\circ} \mathrm{C}$ for $15 \mathrm{~min}$.

\subsection{FAMEs analysis}

Sardine oil, the different fractions obtained by RP-HPLC, the 2-MAG fraction obtained by NP-HPLC and from the bands scraped off from the TLC, were analyzed by gas chromatography to determine the fatty acid profile by the AOAC method (AOAC Official Method 991.39 2000). The fatty acid methyl esters were firstly prepared and then analyzed in a Hewlett Packard gas chromatograph (6890N Network GC System, Santa Clara CA, United States) equipped with an auto-sampler (7683B series) and a flame ionization detector (FID). A fused silica capillary column (OmegawaxTM-320, 30m×0.32mm i.d.) was used. Most of the fatty acid methyl esters were identified by comparison of their retention times with those of chromatographic standards.

\subsection{Calculation of TAG composition}

In order to identify the individual triacylglycerols, the concept of equivalent carbon number (ECN) has been used. The ECN is the carbon number of a hypothetical saturated triacylglycerol, which is eluted at the same retention time as the unsaturated triacylglycerol under study (Podlaha \& Töregård 1982). In a reversed-phase HPLC system, the elution order of the acylglycerols depends on their chain length, degree of unsaturation and the presence of certain functional groups (López-Hernández et al. 2004). Therefore, the ECN value for each TAG is based on these parameters. Different empirical equations have been proposed to predict retention times of TAG species. In literature (Hierro et al. 1992; Najera et al. 1998; Perona \& Ruiz-Gutierrez 1999), the following equation has been used to correlate the dependent variable, $\log k^{\prime}$, related with the retention time of each TAG: 
$\log k^{\prime}=b_{0}+b_{1}^{*}(C N)+b_{2} *(D B)+b_{3} *(N U F A)$

where $\mathrm{CN}$ is the total carbon number of the three fatty acids, DB is the total number of double bonds and NUFA is the number of unsaturated fatty acids of the TAG molecule. Based on equation (1) the ECN value was evaluated according to Equation 2 (Hierro et al. 1992; Najera et al. 1998; Perona \& Ruiz-Gutierrez 1999):

$E C N=C N+a_{1} * D B+a_{2} * N U F A$

Coefficients $a_{1}$ and $a_{2}$ were obtained from the quotient between the coefficients $b_{2}$ and $b_{1}$, and $b_{3}$ and $b_{1}$, respectively.

In this work, instead of equation (1), the equation proposed by López-Hernández et al. (López-Hernández et al. 2004) has been used to correlate the dependent variable, retention time (RT), with the following independent variables $C N, D B, M U F A$ and PUFA:

$R T=b_{0}+b_{1}^{*}(C N)+b_{2}^{*}(D B)+b_{3}^{*}(M U F A)+b_{4}^{*}(P U F A)$

In equation (3) the factor NUFA of equation (1) has been replaced by two different factors: MUFA and PUFA; therefore, the effect of both types of fatty acids, present in high concentrations in fish oils, on elution time is considered. ECN values were evaluated as:

$E C N=C N+a_{1} * D B+a_{2} *$ MUFA $+a_{3} *$ PUFA

where $a_{1}$ is the quotient between the coefficients $b_{2}$ and $b_{1}, a_{2}$ between the coefficients $b_{3}$ and $b_{1}$ and $a_{3}$ between the coefficients $b_{4}$ and $b_{1}$. Retention times have been expressed as relative retention times (RRT) of the peaks with reference to tricaprin peak in the chromatogram. Parameters $\left(b_{0}-b_{4}\right)$ of equation (3) have been obtained by multiple linear regression analyses of relative retention time for the different TAG standards by using the commercial software Statgraphics Centurion XVI.I. ECN values have been related with the RRT by a simple linear regression:

$E C N=a+b * R R T$ 
Identification of TAG in the analysis of fish oil samples has been performed on the basis of their retention characteristics, and their ECN value was calculated through equation (5) to assign TAG to each chromatographic peak.

For each fraction recovered from RP-HPLC a number of fatty acids were obtained by GC. These fatty acids were combined three by three assuming that the three positions on the glycerol molecules were equivalent, since HPLC analysis cannot separate positional isomers. In this procedure only those fatty acids present with an area higher than $5 \%$ have been taken into account. This way, a theoretical ECN value can be calculated (eq. 4) and compared with the experimental value obtained from its characteristic retention time (eq 5) and TAG species could be assigned to the different chromatographic peaks.

\section{Results and discussion}

\subsection{Fatty acid profile of sardine oil}

The FA profile of the original sardine oil by GC-FID is presented in Table A.2. Sardine oil presents high amounts of polyunsaturated fatty acids. The most abundant FA was palmitic acid (around $23 \% \mathrm{~mol})$, followed by eicosapentaenoic acid $(18.3 \% \mathrm{~mol})$ and by palmitoleic and mirystic acids (12.5\% mol). Perona et al. (Perona \& Ruiz-Gutierrez 1999) analyzed the fatty acid profile for sardine oil and found palmitic acid as the most abundant $(21.54 \%)$ followed by oleic acid (15.82\%), eicosapentaenoic acid (14.83\%) and docosahexaenoic acid (13.82\%). The differences found in fatty acid and TAG composition of sardine oil can be expected.

\subsection{Reverse-Phase Liquid chromatographic analyses}

\section{Mixture of standards TAG}

The mixture of TAG standards was clearly separated in the order CCC $<$ DhDhDh $<\operatorname{LnLnLn}$ $<$ LLL $<$ LoLoLo $<$ PoPoPo $<$ MMM $<$ OOO < PPP < SSS < AAA (Fig. A.1). 


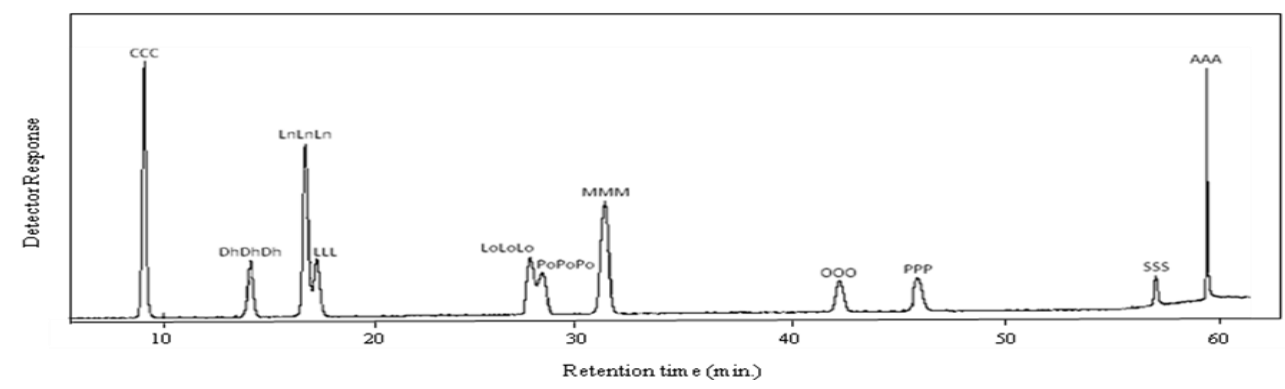

Fig.A.1. RP-HPLC of TAG standards

Table A.2. Fatty acid composition of sardine oil (\% mol) and of $s n-2^{\mathrm{a}}$ of TAG in oils studied in this work

\begin{tabular}{|c|c|c|c|}
\hline \multicolumn{2}{|l|}{ Fatty acid } & TAG & sn-2 \\
\hline Myristic (M) & $14: 0$ & 12.4 & 41.8 \\
\hline Palmitic (P) & $16: 0$ & 22.9 & 41.9 \\
\hline Palmitoleic (Po) & $16: 1 n-7$ & 12.5 & 38.1 \\
\hline Stearic (S) & 18:0 & 3.6 & 6.7 \\
\hline Oleic (O) & $18: 1 n-9$ & 9.8 & 16.6 \\
\hline Vaccenic (V) & $18: 1 n-7$ & 3.7 & 10.1 \\
\hline Linoleic (Lo) & $18: 2 n-6$ & 2.5 & 32.7 \\
\hline Linolenic (Ln) & $18: 3 n-3$ & 1.0 & 29.0 \\
\hline Steriadonic (St) & $18: 4 n-3$ & 3.3 & 26.4 \\
\hline Eicosatrienoic (Et) & $20: 3 n-3$ & 1.3 & 19.6 \\
\hline Eicosapentaenoic (Ep) & $20: 5 n-3$ & 18.3 & 12.1 \\
\hline Docosapentaenoic (Dp) & $22: 5 n-3$ & 1.8 & 76.4 \\
\hline Docosahexaenoic (Dh) & $22: 6 n-3$ & 7.0 & 82.8 \\
\hline
\end{tabular}

(a) $\% s n-2=[\mathrm{mol} \% s n-2$ fatty acid / mol \% fatty acid in TAG.3 $] \cdot 100$

Detector response: The same standard solutions used to determine retention times of the pure TAG were employed to obtain calibration curves for all the standards used in this work. Perona et al. (Perona \& Ruiz-Gutierrez 1999) proposed the use of cubic or fourth regression curves. That means the determination of four or five coefficients in the 
regression analysis. In this work the approach followed by Ruiz-Sala et al. (Ruiz-Sala et al. 1996) has been preferred and linearity has been reached by plotting log area versus log amount injected. Parameters of the regression for all the standards used in this work can be found in Table A.3. Figure A.2 shows the calibration curve of DhDhDh. In general, a good behavior has been observed for the light-scattering detector (ELSD), taking into account its non-linear response (Gotoh et al. 2006; Perona \& Ruiz-Gutierrez 1999).

Table A.3. Parameters of linear regression curves obtained for calibration of standards in RP-HPLC. $\log$ area $=a \cdot(\log$ amount injected $)+b$.

\begin{tabular}{lccc} 
TAG standard & $\mathbf{a}$ & $\mathbf{b}$ & $\mathbf{r}^{2}$ \\
CCC & 1.4522 & 2.524 & 0.9980 \\
LLL & 1.9837 & 2.3262 & 0.9954 \\
MMM & 1.6518 & 2.4137 & 0.991 \\
PPP & 2.8336 & 1.9691 & 0.9975 \\
PoPoPo & 1.6548 & 2.4287 & 0.9998 \\
SSS & 3.7912 & 1.957 & 0.9997 \\
OOO & 1.812 & 2.1294 & 0.9972 \\
LoLoLo & 2.0601 & 1.9225 & 0.9998 \\
LnLnLn & 1.7775 & 2.1997 & 0.9956 \\
AAA & 1.0191 & 0.6597 & 0.9916 \\
DhDhDh & 1.7192 & 2.3328 & 0.9919 \\
\hline
\end{tabular}

\section{$\underline{\text { Sardine oil samples }}$}

The chromatogram of sardine oil is showed in Fig. A.3. Forty four peaks have been detected.

As it has been explained in section 2.2 different fractions were collected from RP-HPLC chromatogram to obtain the different FAME composition of each fraction by GC. Based on Fig.A.3, a total of seven fractions were collected from HPLC. The fatty acid profiles of these fractions are presented in Table A.4. It can be observed that the first fractions (1-3) 
contain most of the highly unsaturated FAME species as eicosapentaenoic, docosapentaenoic and docosahexaenoic acids. In the last fractions, the content of PUFA continuously decreases in each fraction. The highly saturated FAME species, especially palmitic and stearic acids, appear mainly in the last fractions (5-7). Concentration of linolenic and eicosatrienoic acids was lower than $2 \%$ in the FAME analysis of sardine oil; for this reason these fatty acids were nearly absent in the analysis of fractions.

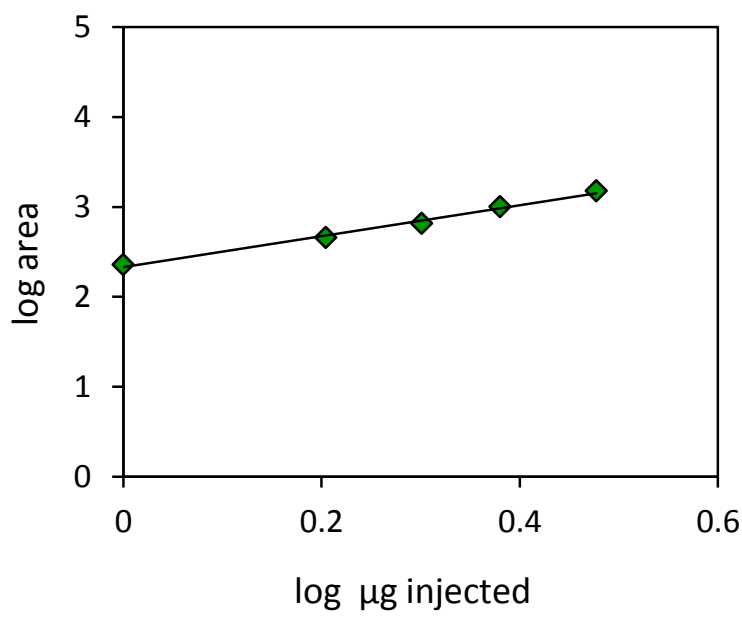

Fig.A.2. Calibration curve for DhDhDh standard analyzed by RP-HPLC. The continuous line corresponds to the linear regression. Log area $=1.7192 \cdot(\log \mu g$ injected $)+2.3328$

The fatty acid profiles of each fraction would help to predict the most probable TAG composition for the sardine oil studied in this work, without regard of the regiospecificity of each fatty acid in the glycerol molecule.

\subsection{Qualitative analysis of TAG composition of sardine oil}

\section{Calculation of TAG composition}

Multiple linear regression analysis has been performed to obtain the parameters of equation (3) using the relative retention time of TAG standards. When all the standards were included, the correlation was not very successful. To improve data correlation, TAG 
species of little relevance in fish oil, in this case trilaurin and triarachidin were excluded in the fitting procedure. The same behavior in the fitting procedure has been observed by López-Hernández (López-Hernández et al. 2004) in the analysis of the TAG composition of reaction mixture of hydrogenation of fish oil with hydrogenated soybean oil. Nonlinear multiple regression analysis leads the following values of the model parameters:

RRT $=-64.228+2.138 \cdot \mathrm{CN}-3.360 \cdot \mathrm{DB}-1.877 \cdot \mathrm{MUFA}-3.571 \cdot \mathrm{PUFA}$

Fig.A.3. TAG analysis of sardine oil using the gradient solvent RP-HPLC technique. Numbers correspond to the peaks of Table 7.

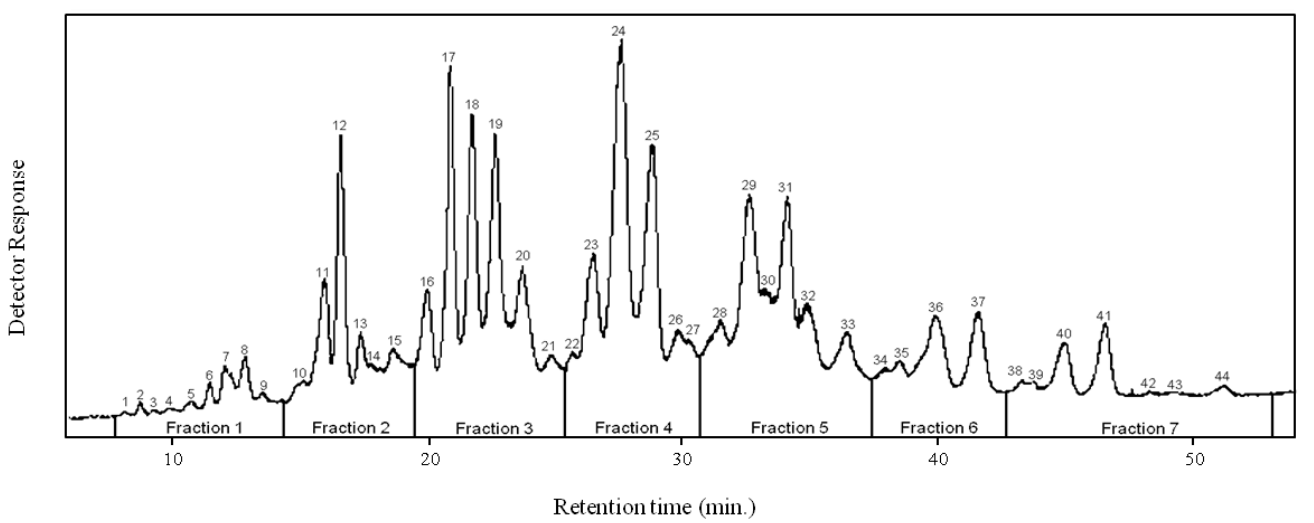

As it has been described in section 2.3, using the parameters from equation (6), the following equation for the ECN value has been obtained:

$E C N=C N-1.572 \cdot D B-0.878 \cdot M U F A-1.670 \cdot P U F A$

Table A.5 shows the ECN values obtained with equation (7) for the different TAG standards used in this work. It can be observed that the elution order agrees with the ECN value of each TAG.

When equation (1) was used to correlate the experimental retention times of standards TAG, equation (8) was obtained to evaluate the ECN values:

$\mathrm{ECN}=\mathrm{CN}-1.572 \cdot \mathrm{DB}-0.878 \cdot \mathrm{NUFA}$ 
In this expression the effect of MUFA and PUFA on retention time is not distinguished.

Table A.5 also shows the ECN values obtained with equation (8). In this case, the order of the ECN values does not correspond with their retention times. Therefore when no addition factor is considered for the long chain polyunsaturated fatty acids, ECN values do not longer correlate with the elution time.

Table A.4. Fatty acid composition $(\% \mathrm{~mol})$ of sardine oil fractions

\begin{tabular}{|c|c|c|c|c|c|c|c|}
\hline Fatty acid & 1 & 2 & 3 & 4 & 5 & 6 & 7 \\
\hline Myristic (M) & 14.09 & 27.72 & 13.11 & 18.51 & 11.85 & 12.58 & 10.56 \\
\hline Palmitic (P) & 18.27 & 14.94 & 17.73 & 26.28 & 34.76 & 32.11 & 28.52 \\
\hline Palmitoleic (Po) & 12.95 & 25.31 & 12.93 & 18.62 & 11.37 & 12.56 & 8.29 \\
\hline Stearic (S) & 7.36 & - & 1.49 & 3.91 & 4.06 & 7.69 & 12.50 \\
\hline Oleic (O) & 13.75 & 6.95 & 4.29 & 8.43 & 13.59 & 16.23 & 22.02 \\
\hline Vaccenic (V) & - & - & 2.45 & 4.57 & 5.81 & 4.86 & 8.54 \\
\hline Linoleic (Lo) & 10.32 & 4.12 & 1.85 & 3.84 & 3.49 & 3.26 & 9.56 \\
\hline Linolenic (Ln) & - & - & 1.51 & - & - & - & - \\
\hline Steriadonic (St) & 4.62 & 3.40 & 4.57 & 1.75 & 1.70 & - & - \\
\hline Eicosatrienoic (Et) & - & - & 2.03 & - & - & 1.44 & - \\
\hline Eicosapentaenoic (Ep) & 11.36 & 15.41 & 25.46 & 8.84 & 8.30 & 3.99 & - \\
\hline Docosapentaenoic (Dp) & 2.91 & - & 2.83 & 1.67 & 1.29 & 1.73 & - \\
\hline Docosahexaenoic (Dh) & 4.37 & 2.15 & 9.75 & 3.57 & 3.78 & 3.55 & - \\
\hline $\begin{array}{l}\text { \% area of each fraction } \\
\text { (RP-HPLC) }\end{array}$ & 3.6 & 8.1 & 19.5 & 23.8 & 19.0 & 12.8 & 13.2 \\
\hline
\end{tabular}

From the ECN values for standards TAG, it is possible to derive partial ECN values for each individual acid component of triacylglycerol, since these partial values seem to be additive and it is possible to calculate by addition the ECN values for unknown triacylglycerols (Svensson \& Adlercreutz 2008). The partial ECN values for fatty acids are presented in Table A.6 for the standards used in this work. This table also shows ECN values found in the literature for these fatty acids (Podlaha \& Töregård 1982; Podlaha \& Töregård 1989; Rezanka \& Mares 1991; Svensson \& Adlercreutz 2008). The ECN value for all the fatty acids 
present in sardine oil could not be directly determined since no triacylglycerol standards were available in our laboratory. These ECN values were obtained from equation (7) and are also presented in Table A.6. Therefore, these values are, to some extent, uncertain. Nevertheless these values will be used to calculate the ECN values from the unknown triacylglycerols of sardine oil.

Table A.5. ECN values of TAG standards obtained with different equations

\begin{tabular}{lcc} 
TAG standard & ECN (eq.7) & ECN (eq.8) \\
CCC & 30.00 & 30.00 \\
DhDhDh & 32.70 & 35.08 \\
LnLnLn & 34.84 & 37.22 \\
LoLoLo & 39.56 & 41.94 \\
PoPoPo & 40.65 & 40.65 \\
MMM & 42.00 & 42.00 \\
OOO & 46.65 & 46.65 \\
PPP & 48.00 & 48.00 \\
SSS & 54.00 & 54.00 \\
\hline
\end{tabular}

For each chromatographic peak obtained in the analysis of sardine oil, experimental ECN values were calculated from their relative retention times by using the linear regression obtained for the ECN standard TAG and their relative retention times (Fig. A.4):

$E C N=0.465 \cdot R R T+30.111$

To determine a tentative composition of TAG in the sardine oil studied in this work, experimental ECN values for each chromatographic peak (eq. 9) have been compared with theoretical ECN values of TAG. This theoretical ECN value has been obtained combining three by three the different fatty acid composition obtained by GC analysis of each fraction collected by RP-HPLC. The ECN error was estimated \pm 0.3 (from three chromatograms). Usually, two or even more TAG can be assigned to a single chromatographic peak since different combinations of fatty acids can give the same ECN 
value. In these cases, TAG formed with fatty acids with less than $5 \%$ area percentage in the GC analysis of each fraction have not been included in the tentative composition of sardine oil. When TAG peaks were not identified, fatty acids with more than $4 \%$ area were also taken into account. Table A.7 presents the compositional results of predicted TAG species of the seven fractions obtained by RP-HPLC. From this table it can be observed that around $60 \%$ of TAG molecular species were bound to one or more polyunsaturated fatty acids.

TableA.6. Partial ECN values for the unsaturated fatty acids obtained with equation (7) together with others values found in the literature

\begin{tabular}{|c|c|c|c|c|c|}
\hline Fatty acid & $\begin{array}{l}\text { ECN1, } \\
\text { eq.(7) }\end{array}$ & $\begin{array}{c}\text { ECN2 } \\
\text { (Podlaha \& } \\
\text { Töregård } \\
\text { 1982) }\end{array}$ & $\begin{array}{c}\text { ECN3 } \\
\text { (Svensson \& } \\
\text { Adlercreutz } \\
\text { 2008) }\end{array}$ & $\begin{array}{c}\text { ECN4 } \\
\text { (Podlaha \& } \\
\text { Töregård } \\
\text { 1989) }\end{array}$ & $\begin{array}{c}\text { ECN5 } \\
\text { (Rezanka } \\
\text { \& Mares } \\
\text { 1991) }\end{array}$ \\
\hline Palmitoleic & 13.55 & 13.12 & - & 13.12 & - \\
\hline Oleic & 15.55 & 15.00 & 15.60 & 15.05 & 15.42 \\
\hline Linoleic & 13.19 & 12.38 & 13.10 & 12.73 & 12.84 \\
\hline Linolenic & 11.61 & 10.75 & 11.10 & 10.81 & 10.40 \\
\hline Docosahexaenoic & 10.90 & - & - & - & - \\
\hline Steriadonic & 10.04 & & & & \\
\hline Eicosenoic & 17.55 & & & & \\
\hline Eicosatrienoic & 13.61 & & & & \\
\hline Eicosapentaenoic & 10.47 & & & & \\
\hline Docosapentaenoic & 12.47 & & & & \\
\hline
\end{tabular}

Distribution of FA in sn-2 and sn-1 (or sn-3) positions

In order to predict in a better way the proportional distribution of fatty acids, ethanolysis of sardine oil has been carried out under the experimental conditions described in section 2.2. After $4 \mathrm{~h}$ a maximum in the 2-MAG production has been described (Shimada et al. 2003). The amount of 2-MAG for sardine oil was $22.0 \%$. FAME analysis for 2-MAG separated by TLC and NP-HPLC has been conducted to determine FA bound to sn-2 
position. Both techniques exhibited similar findings without significant differences. Table A.2 shows the results obtained in the regiospecific analysis. Results of FA distribution in sn-2 position are expressed as(Jayasinghe et al. 2012):

$\%$ sn-2 = [mol \% sn-2 fatty acid $/ \mathrm{mol} \%$ fatty acid in TAG.3 $] \cdot 100$

sn-1 (or sn-3) positions can be easily calculated as:

$\% s n-1($ or $s n-3)=100-[\% s n-2]$

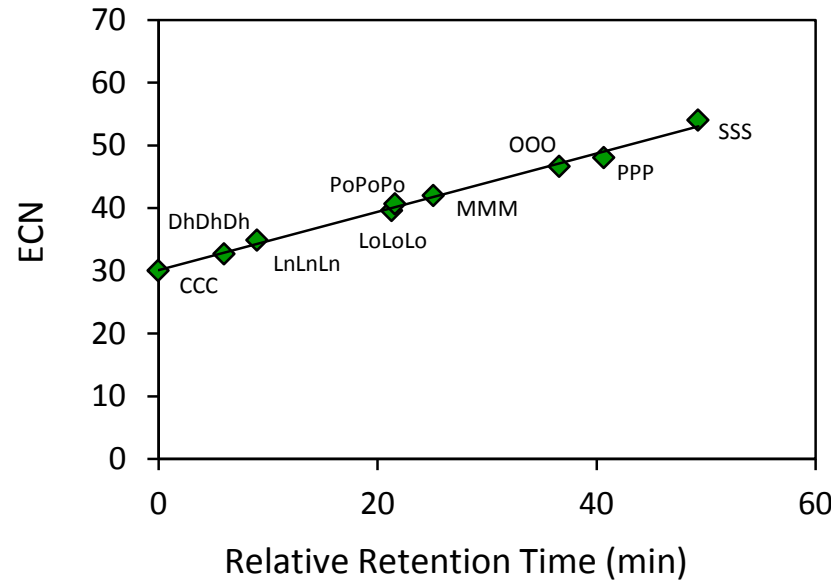

Fig.A.4. Correlation between ECN (equation 7) and RRT of TAG standards.

$$
\mathrm{ECN}=0.4647 \cdot(\mathrm{RRT})+30.111
$$

From Table A.2, it can be observed that docosahexaenoic acid is mostly found at sn-2 position. This result agrees with recent studies in the regiospecific analysis of fish oil triacylglycerols (Ando \& Narukawa 2002; Jayasinghe et al. 2012).

Furthermore, myristic, palmitic, palmitoleic, linoleic, linolenic and steriadonic acids are randomly distributed in sn-1 (or sn-3) and sn-2 positions (26.4-41.9\% mol). On the other hand, stearic, oleic, vaccenic, eicosatrienoic and eicosapentaenoic acids are hardly bound to the sn-2 position $(6.7-19.6 \% \mathrm{~mol})$. 


\section{Principal component analysis}

Seven fractions (objects) have been collected in the analysis of sardine oil by RP-HPLC and their corresponding fatty acid composition has been considered the chemical descriptors.

To visualize the trends of the data, the scores for samples and the loadings for variables were represented in the space of the two principal components (PCS) obtained from PCA (Diaz et al. 2005). Figure A.5 shows the loading and score plots of the two principal components for the differences between the fatty acid compositions of the fractions. Loading plots in the planes PC1-PC2 and PC1-PC3 reveal that all of the fatty acids variables give their variance to PC1. For this reason only planes PC1-PC2 are plotted.

The first fractions (1-4) are located on the right side of the graph, where the PC1 presents positive values. However, the last fractions (5-7) are located on the left side of the graphs, where the PC1 shows negative values. Polyunsaturated fatty acids, myristic and palmitoleic acids variables present large and positive loadings, regarding PC1 while saturated and monounsaturated fatty acids variables exhibit large and negative loadings.

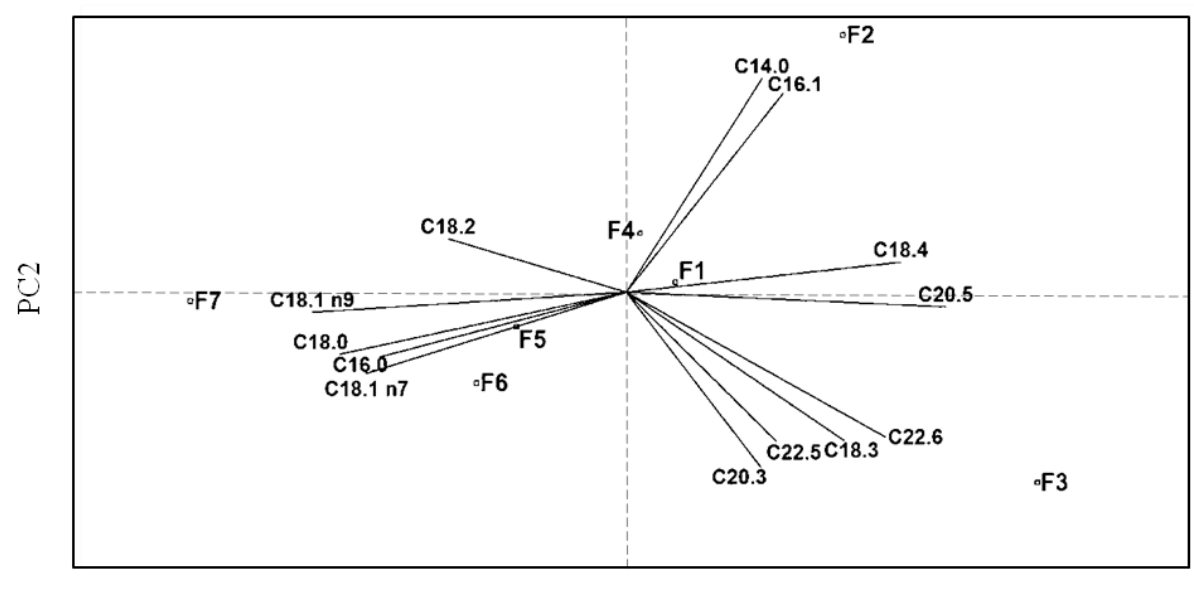

$\mathrm{PCl}$

Fig.A.5. Loadings and scores plot from PCA of data about fatty acids of fractions for sardine oil, in the PC1-PC2 planes 
Table A.7. Predicted TAG species from HPLC peaks present in area $>0.2 \%$ of total in sardine oil

\begin{tabular}{|c|c|c|c|c|c|c|}
\hline Fraction & $\begin{array}{l}\text { PeakN } \\
\text { o. }\end{array}$ & RRT & $\begin{array}{l}\text { Experi- } \\
\text { mental } \\
\text { ECN(Eq.9) }\end{array}$ & Predicted TAG & $\begin{array}{c}\% \\
\text { Area }\end{array}$ & $\begin{array}{l}\text { Predicted } \\
\text { ECN (Eq. 7) }\end{array}$ \\
\hline \multirow{9}{*}{1} & 1 & -0.05 & 30.1 & St-St-St & 0.24 & 30.1 \\
\hline & 2 & 0.72 & 30.5 & St-St-Ep & 0.53 & 30.6 \\
\hline & 3 & 1.18 & 30.7 & St-St-Ep & 0.21 & 30.6 \\
\hline & 4 & 1.53 & 30.8 & St-St-Dh / St-Ep-Ep & 0.20 & 31.0 \\
\hline & 5 & 2.78 & 31.4 & Ep-Ep-Ep & 0.25 & 31.4 \\
\hline & 6 & 3.29 & 31.6 & Ep-Ep-Ep / Ep-Ep-Dh & 0.38 & $31.4-31.8$ \\
\hline & 7 & 4.19 & 32.1 & Ep-Ep-Dh / Ep-Dh-Dh & 0.79 & $31.8-32.3$ \\
\hline & 8 & 4.63 & 32.3 & Ep-Dh-Dh & 0.91 & 32.3 \\
\hline & 9 & 5.48 & 32.7 & Dh-Dh-Dh & 0.35 & 32.7 \\
\hline \multirow{6}{*}{2} & 10 & 6.9 & 33.3 & Lo-St-St & 0.42 & 33.3 \\
\hline & 11 & 7.73 & 33.7 & Po-St-St / Lo-St-Ep & 1.75 & $33.6-33.7$ \\
\hline & 12 & 8.4 & 34.0 & Po-St-Ep & 4.47 & 34.1 \\
\hline & 13 & 9.21 & 34.4 & Po-Ep-Ep & 0.75 & 34.5 \\
\hline & 14 & 9.46 & 34.5 & Po-Ep-Ep & 0.27 & 34.5 \\
\hline & 15 & 10.48 & 35.0 & M-Ep-Ep & 0.95 & 34.9 \\
\hline \multirow{6}{*}{3} & 16 & 11.69 & 35.5 & Po-Dh-Dh / M-Ep-Dh / M-Dh-Dh & 1.85 & $35.4-35.8$ \\
\hline & 17 & 12.53 & 35.9 & M-Dh-Dh & 7.57 & 35.8 \\
\hline & 18 & 13.47 & 36.4 & $\begin{array}{c}\text { O-St-Ep / P-St-St / O-Ep-Ep / O- } \\
\text { St-Dh / P-St-Ep }\end{array}$ & 4.08 & $36.1-36.5$ \\
\hline & 19 & 14.26 & 36.7 & P-Ep-Ep & 4.09 & 36.9 \\
\hline & 20 & 15.15 & 37.2 & P-Ep-Ep / M-M-Dh / P-Ep-Dh & 0.83 & $36.9-37.4$ \\
\hline & 21 & 15.59 & 37.4 & M-M-Dh / P-Ep-Dh / Po-Po-Ep & 0.61 & $37.2-37.6$ \\
\hline \multirow{6}{*}{4} & 22 & 16.57 & 37.8 & $\begin{array}{c}\text { Po-Po-Ep / P-Dh- Dh / Po-Po-Dh / } \\
\text { M-Po-Ep }\end{array}$ & 0.46 & $37.6-38.0$ \\
\hline & 23 & 18.14 & 38.5 & M-Po-Dh / M-M-Ep & 2.24 & $38.4-38.5$ \\
\hline & 24 & 19.11 & 39.0 & S-Ep-Ep / O*-Lo-Ep & 12.4 & $39.0-39.2$ \\
\hline & 25 & 20.14 & 39.5 & Po-O*-Ep & 7.54 & 39.6 \\
\hline & 26 & 21.36 & 40.0 & Po-O*-Dh / M-O*-Ep / P-Po-Ep & 0.54 & 40.0 \\
\hline & 27 & 21.49 & 40.1 & Po-O*-Dh / M-O*-Ep / P-Po-Ep & 0.29 & 40.0 \\
\hline \multirow{6}{*}{5} & 28 & 22.64 & 40.6 & $\begin{array}{c}\text { M-O*-Dh / P-Po-Dh / M-P-Ep / } \\
\text { M-P-Dh }\end{array}$ & 1.90 & $40.4-40.9$ \\
\hline & 29 & 23.95 & 41.2 & M-Po-Po & 8.38 & 41.1 \\
\hline & 30 & 25.15 & 41.8 & $\begin{array}{c}\text { M-M-Po / O*-O*-Ep / M-M-M / } \\
\text { O*-O*-Dh / P-O*-Ep }\end{array}$ & 0.65 & $41.6-42.0$ \\
\hline & 31 & 25.63 & 42.0 & M-M-M / O*-O*-Dh / P-O*-Ep & 3.23 & 42.0 \\
\hline & 32 & 26.37 & 42.4 & P-O*-Dh / P-P-Ep / Po-Po-O* & 1.55 & $42.4-42.7$ \\
\hline & 33 & 27.64 & 43.0 & P-P-Dh / M-Po-O* / P-Po-Po & 3.24 & $42.9-43.1$ \\
\hline
\end{tabular}




\begin{tabular}{|c|c|c|c|c|c|c|}
\hline \multirow{4}{*}{6} & 34 & 28.86 & 43.5 & M-M-O* / M-P-Po & 0.55 & 43.6 \\
\hline & 35 & 30.10 & 44.1 & M-M-P / S-O*-Ep & 0.87 & 44.0 \\
\hline & 36 & 31.20 & 44.6 & P-S-Ep / Po-O*-O* & 4.83 & $44.5-44.7$ \\
\hline & 37 & 32.61 & 45.3 & $\begin{array}{c}\text { M-O*-O* / P-Po-O* / Po-Po-S / } \\
\text { M-P-O* / M-Po-S / P-P-Po }\end{array}$ & 6.52 & $45.1-45.6$ \\
\hline \multirow{7}{*}{7} & 38 & 34.06 & 45.9 & M-M-S /M-P-P & 0.64 & 46.0 \\
\hline & 39 & 35.24 & 46.5 & O*-O*-O* /S-O*-Lo & 0.56 & 46.7 \\
\hline & 40 & 35.99 & 46.8 & $\begin{array}{c}\text { O*-O*-O* /S-O*-Lo / P-O*-O* / } \\
\text { Po-S-O* }\end{array}$ & 3.79 & $46.7-47.1$ \\
\hline & 41 & 37.36 & 47.5 & $\begin{array}{c}\text { P-S-Lo / S-S-Lo / M-S-O* /P-P-O* } \\
\text { / P-Po-S }\end{array}$ & 5.93 & $47.2-47.6$ \\
\hline & 42 & 38.80 & 48.1 & M-P-S / P-P-P & 0.43 & 48.0 \\
\hline & 43 & 40.30 & 48.8 & $\mathrm{~S}-\mathrm{O} *-\mathrm{O} *$ & 0.48 & 49.1 \\
\hline & 44 & 41.74 & 49.5 & P-S-O* / Po-S-S & 1.44 & 49.6 \\
\hline
\end{tabular}

Fatty acids: $\mathrm{M}=$ myristic acid (14:0); $\mathrm{P}=$ palmitic acid (16:0); $\mathrm{Po}=$ palmitoleic acid (16:1); $\mathrm{S}=$ stearic acid (18:0); O=oleic acid (18:1); Lo= linoleic acid (18:2); Ln=linolenic acid (18:3); St=stearidonic acid (18:4); Et=eicosatrienoic acid (20:3); Ep=eicosapentaenoic acid (20:5); Dp=docosapentaenoic acid (22:5); $\mathrm{Dh}=$ docosahexaenoic acid (22:6).

* Oleic acid in the corresponding TAG could be also vaccenic acid, both acids show the same carbon number, double bonds and number of MUFA; therefore it is not possible to distinguish them.

PC1 and PC2 were able to describe respectively $51.59 \%$ and $25.82 \%$ of total variance, while PC3 described $13.06 \%$. Moreover, those variables whose loadings present small angles are correlated, positively or negatively, according to their position in the plot. For instance, myristic and palmitoleic acids present positive correlation. Namely, when one grows, the other grows too. Otherwise, palmitic, stearic and vaccenic acids present negative correlation; so when one increases, the others decrease. This type of correlations have been also observed when predicting a probably TAG composition of sardine oil. For instance, from Table A.7, it can be observed, in the first fractions, TAG formed by combination of $C 16: 1$ and $C 20: 5 n-3$ or $C 22: 6 n-3$ as well as TAG with $C 14: 0$ and $C 20: 5 n-3$ or $\mathrm{C} 22: 6 n-3$; that is, when $\mathrm{C} 14: 0$ is present, $\mathrm{C} 20: 5 n-3$ or $\mathrm{C} 22: 6 n-3$ will be also present in the same TAG. Therefore, PCA can be a useful tool to verify TAG composition calculated in

\section{Table A.7.}




\section{Conclusions}

In this work, HPLC combined with GC has been used to analyze the triacylglycerol composition of sardine oil.

The equation obtained through a multiple linear regression analysis, with the relative retention times of TAG standards, is able to provide good ECN values to indentify the majority of TAG molecular species present in very complex mixtures as those from sardine oil. Moreover, the PCA analysis provides a versatile tool to assurance the most probably TAG contents.

As expected from the FA profile, the major TAG species present in sardine oil were rich in docosahexaenoic, eicosapentaenoic, and docosapentaenoic acids. Regiospecific analysis of FA in the TAG by ethanolysis with Candida antarctica shows that docosahexaenoic acid is mainly bound at sn-2 position. The present findings explore the possibilities of utilizing these oils as a PUFA-rich source in fish oil industry. 

APPENDIX B

Analytical methods 



\section{Analytical methods}

This appendix is focused on the main analytical methods used in this work for characterization of the initial sardine oil and determination of the reaction products for the kinetic studies. The methods applied for the evaluation of the oxidation status of the oil samples are also included.

The main objective of this appendix is to expand the analytical methods information, which have been briefly describe in the chapters, to facilitate their understanding and practical application. A systematic description of each method and its operational aspects is presented.

The analytical methods that have been developed are the following:

I. Determination of fatty acid profile

II. Determination of neutral lipid profile

III. Determination of peroxide value (PV)

IV. Determination of anisidine value (AV)

V. Analysis of Thiobarbituric Acid Reactive Substances (TBARS) 



\section{Fatty acid profile}

The fatty acid profile of sardine oil and lipid fractions was determined qualitatively and quantitatively by gas chromatography (GC) following the AOAC Method (AOAC Official Method 991.39 2000). This method required a previous derivatization to transform the fatty acids to methyl esters to be analyzed by GC. Identification and quantification of fatty acids was carried out by comparison of retention times and peak areas according to standard compounds, using methyl tricosanoate as internal standard.

\section{Equipment}

The equipment used in this analysis was an Agilent Gas Chromatograph (6890N Network GC System) equipped with an auto-sampler (7683B series) and a flame ionization detector (FID). The column used was a fused silica capillary column, specifically OmegawaxTM-320, $30 \mathrm{~m} \times 0.32 \mathrm{~mm}$ i.d

\section{Reagents}

- Isooctane solution of methyl tricosanoate $(\mathrm{C} 23: 0)$ of $1 \mathrm{mg} / \mathrm{mL}$ as internal standard (IS)

- $\quad 0.5 \mathrm{M}$ methanolic sodium hydroxide $(\mathrm{NaOH})$

- $\quad$ Boron trifluoride $\left(\mathrm{BF}_{3}\right), 12 \%$ in methanol

- $\quad$ Saturate salt solution ( $36 \mathrm{~g} \mathrm{NaCl} / 100 \mathrm{~mL}$ of water)

- $\quad$ Fatty acids methyl esters standards.

\section{Experimental procedure}

\section{- Sample preparation: derivatization process}

A scheme of the derivatization process is presented in Figure B.1. Acylglycerides samples (TAG, DAG and MAG) require the complete derivatization process to transform the fatty acids to methyl esters (FAMEs). In case of free fatty acid samples, the first methylation step with $\mathrm{NaOH}$ methanolic is not required. This is a mild methylation, where the fatty 
acids are disengaged from the glycerol structure to be available for the strong methylation step, with the $\mathrm{BF}_{3}$ as methylate reagent.

When ethyl esters samples were analyzed this derivatization process would not be required; however the retention times of the compounds in GC-FID will be slightly different from the corresponding methyl esters, so the peak identification would be complicated if methyl esters retention times have only been established.

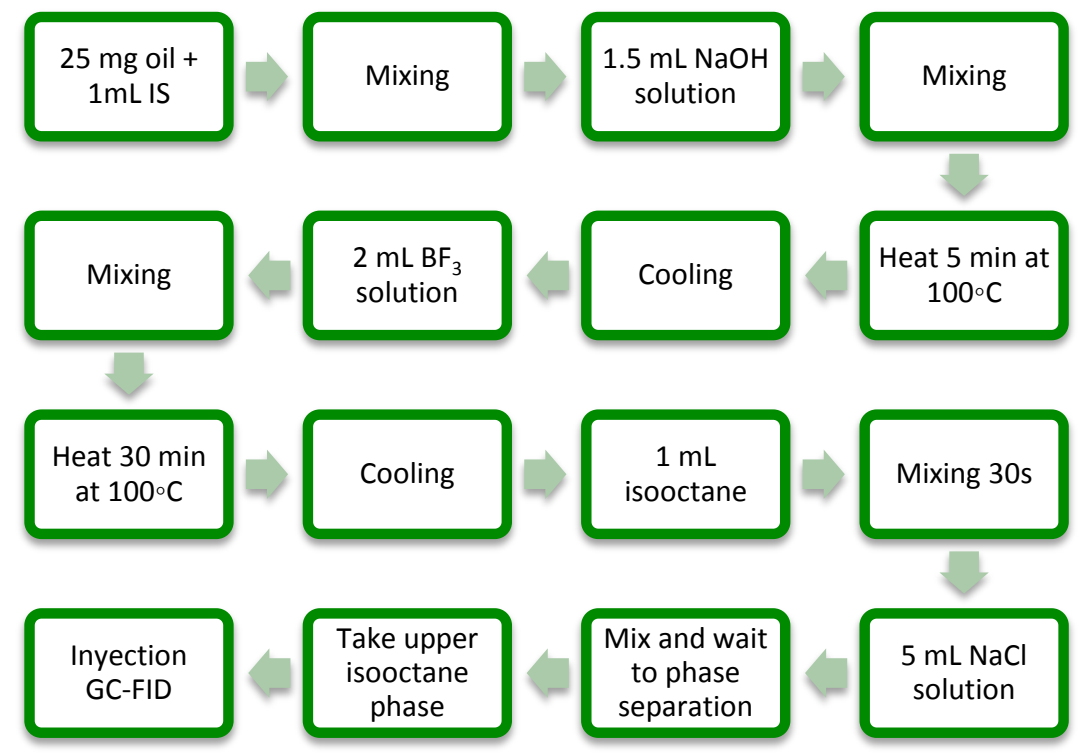

Figure B.1. Derivatization process for the analysis of fatty acids in oil samples.

\section{- Chromatographic method}

The fatty acids methyl esters contained in the isooctane phase were transferred into a glass vial for auto-sampler injection in the GC-FID. The conditions of the chromatographic method are detailed in the table below, Table B.1. 
Table B.1. Conditions of the chromatographic method used in this work for the analysis of fatty acid methyl esters.

\begin{tabular}{|c|c|c|c|}
\hline Injection volume & $1 \mu \mathrm{L}$ & & \\
\hline Injector temperatura & $250^{\circ} \mathrm{C}$ & & \\
\hline Split injection & $1: 50$ & & \\
\hline \multirow{3}{*}{ Flow carrier gas } & $1.8 \mathrm{~mL} / \mathrm{min}$ of & & \\
\hline & helium & & \\
\hline & Rate $\left({ }^{\circ} \mathrm{C} / \mathrm{min}\right)$ & $\mathrm{T}\left({ }^{\circ} \mathrm{C}\right)$ & $\underline{\text { Hold time (min) }}$ \\
\hline Oven temperature & - & 180 & 20 \\
\hline \multirow[t]{2}{*}{ program } & 1 & 200 & 1 \\
\hline & 5 & 220 & 20 \\
\hline Run time & $65 \mathrm{~min}$ & & \\
\hline FID temperature & $250^{\circ} \mathrm{C}$ & & \\
\hline \multirow{3}{*}{ Flows of FID gases } & Hvdrogen & Svnthetic air & Nitrogen (make- \\
\hline & & & $\underline{\text { up gas) }}$ \\
\hline & $40 \mathrm{~mL} / \mathrm{min}$ & $40 \mathrm{~mL} / \mathrm{min}$ & $20 \mathrm{~mL} / \mathrm{min}$ \\
\hline
\end{tabular}

Identification of chromatographic peaks

Fatty acids were identified by means of standard FAMEs comparing their retention times. Table B.2 includes the main fatty acids ordered according to their retention times. As it can be seen in the table, Omegawax column separates the FAMEs by the carbon number and number of double bonds. 
Table B.2. Retention times of fatty acid methyl esters found in sardine oil.

\begin{tabular}{lcc} 
Fatty acid (as methyl ester) & Chemical formula & Retention time (min) \\
Myristic acid & C14:0 & 3.9 \\
Palmitic acid & $\mathrm{C} 16: 0$ & 6.4 \\
Palmitoleic acid & $\mathrm{C} 16: 1 \mathrm{n}-7$ & 6.9 \\
Stearic acid & $\mathrm{C} 18: 0$ & 11.5 \\
Oleic acid & $\mathrm{C} 18: 1 \mathrm{n}-9$ & 12.3 \\
Vaccenic acid & $18: 1 \mathrm{n}-7$ & 12.5 \\
Linoleic acid & $\mathrm{C} 18: 2 \mathrm{n}-6$ & 14.0 \\
$\boldsymbol{\alpha}-$ Linolenic acid & $\mathrm{C} 18: 3 \mathrm{n}-3$ & 17.1 \\
Stearidonic acid & $\mathrm{C} 18: 4 \mathrm{n}-3$ & 18.9 \\
Eicosatrienoic acid & $\mathrm{C} 20: 3 \mathrm{n}-3$ & 29.2 \\
Eicosapentaenoic acid & $\mathrm{C} 20: 5 \mathrm{n}-3$ & 33.9 \\
Docosapentaenoic acid & $\mathrm{C} 22: 5 \mathrm{n}-3$ & 46.5 \\
Docosahexaenoic acid & $\mathrm{C} 22: 6 \mathrm{n}-3$ & 47.9 \\
\hline
\end{tabular}

Figure B.1 shows the chromatogram with the fatty acids detected in the initial refined sardine oil used in this work.

\section{Quantification of fatty acids}

The amount of each fatty acid in the oil sample is determined according to Equation B.1:

$$
m g F A /_{g} O I L=\frac{\text { Area }_{F A} \times \text { Weight }_{I S}}{\text { Area }_{I S} \times \text { Weight }_{O I L} \times S C C_{F A} \times C F_{E-A}} \times 1000
$$

where the weight of IS and the weight of oil is expressed in $\mathrm{mg}, \mathrm{SCC}_{\mathrm{FA}}$ corresponds to the slope of the calibration curve of each standard compound and $\mathrm{CF}_{\mathrm{E}-\mathrm{A}}$ is the ratio between the molecular weight of methyl ester and the molecular weight of fatty acid in each case, 
this conversion factor is necessary to express the result as mg of fatty acid / $\mathrm{g}$ of oil (rather than as methyl ester).

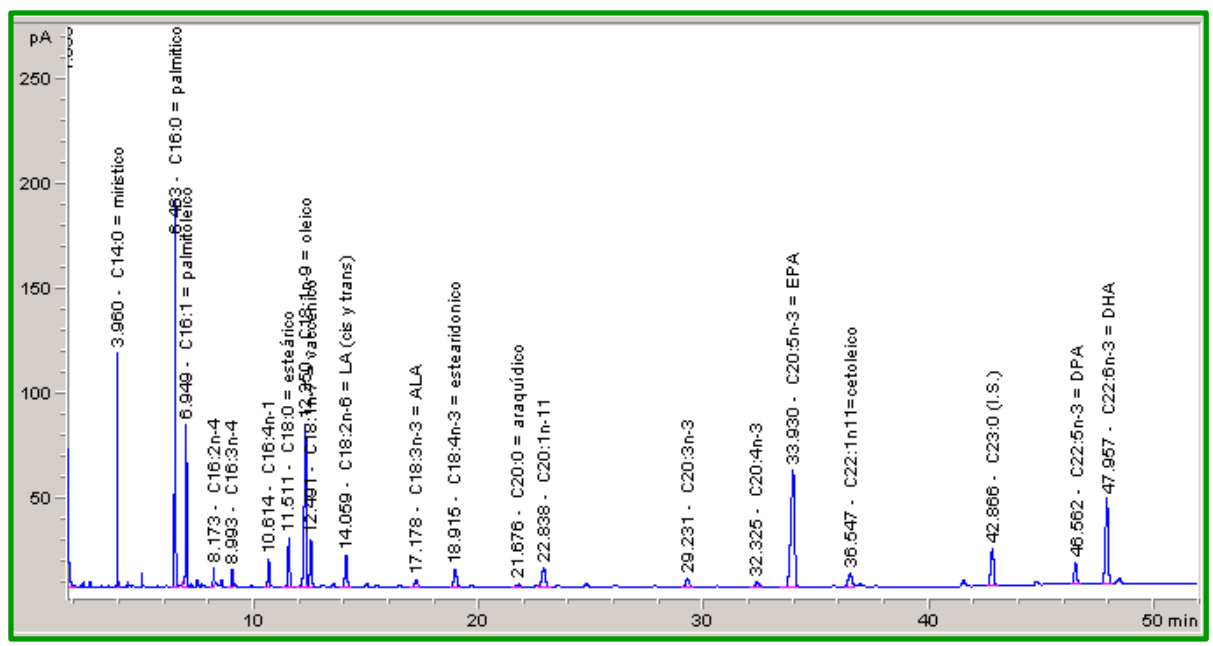

Figure B.1. GC-FID chromatogram of fatty acids in sardine oil (as methyl esters). 


\section{Neutral lipid profile}

The neutral lipids profile (TAG, DAG, MAG and FFA) of sardine oil and reaction products samples were determined qualitatively and quantitatively by a Normal-Phase High Performance Liquid Chromatography (NP-HPLC) following a procedure based on the method proposed by Rubio-Rodriguez et al. and Schaefer et al. (Rubio-Rodríguez et al. 2012; Schaefer et al. 2003). In normal-phase chromatography, the stationary phase is polar and the mobile phase is nonpolar, so the least polar compounds elute first and the most polar compounds elute last. It presents important advantages such as low system back pressure and easy solvent evaporation. The interaction force not only depends on the functional groups of the compound but also on steric factors so structural isomers can be separated.

\section{Equipment}

The chromatographic apparatus consisted of a HPLC system (Agilent 1200) equipped with an automatic degasser, a quaternary pump and an auto-injector. The chromatographic separation of the compounds was performed on a LiChrospher ${ }^{\circledR}$ Diol column $(5 \mu \mathrm{m}, 4 \mathrm{~mm}$ $\times 250 \mathrm{~mm}$ ). The detection was performed in an evaporative light scattering detector, ELSD (Agilent 1200 series).

\section{Reagents}

- Isooctane HPLC grade

- $\quad$ Mixture of methyl tert-butyl ether (MTBE) and acetic acid (99.9/0.1)

- Standards:

MAG: 1-monopalmitin, 1-monoolein, 2-monoolein and 1-monodocosahexaenoin DAG: dipalmitin and diolein (mix of both isomers)

FFA: oleic acid and palmitic acid 


\section{Experimental procedure}

- Sample preparation

Samples were dissolved in tert-pentanol and diluted up to vial concentration of $1 \mathrm{mg} / \mathrm{mL}$ in case of oil samples and of $5 \mathrm{mg} / \mathrm{mL}$ in case of reaction products samples. Diluted samples can be injected directly into the HPLC or previously filtered through a microfilter $(0.45 \mu \mathrm{m})$ if necessary.

\section{- Chromatographic method}

Neutral lipid separation was carried out at room temperature in the normal phase column (LiChrospher ${ }^{\circledR}$ Diol-5) and a flow rate of $1 \mathrm{~mL} / \mathrm{min}$ with $(A)$ isooctane and (B) MTBE/acetic acid $(99.9 / 0.1, \mathrm{v} / \mathrm{v})$ using the binary solvent gradient presented in Table B.3. The detection was performed by ELS at $35^{\circ} \mathrm{C}, 3.5$ bar of nitrogen as nebulizer gas and gain 7 .

Table B.3. Binary solvent gradient used in neutral lipid analysis by HPLC-ELSD

\begin{tabular}{cc} 
Time $(\mathrm{min})$ & \% Mobile phase B \\
0 & 0 \\
1 & 0 \\
10 & 10 \\
22 & 44 \\
30 & 100 \\
42 & 80 \\
47 & 0 \\
\hline
\end{tabular}

Identification of chromatographic peaks

The neutral lipids families were identified by means of neutral lipid standards comparing their retention times. Figure B.2 shows a HPLC-ELSD chromatogram with the neutral lipid classes detected by this method. 


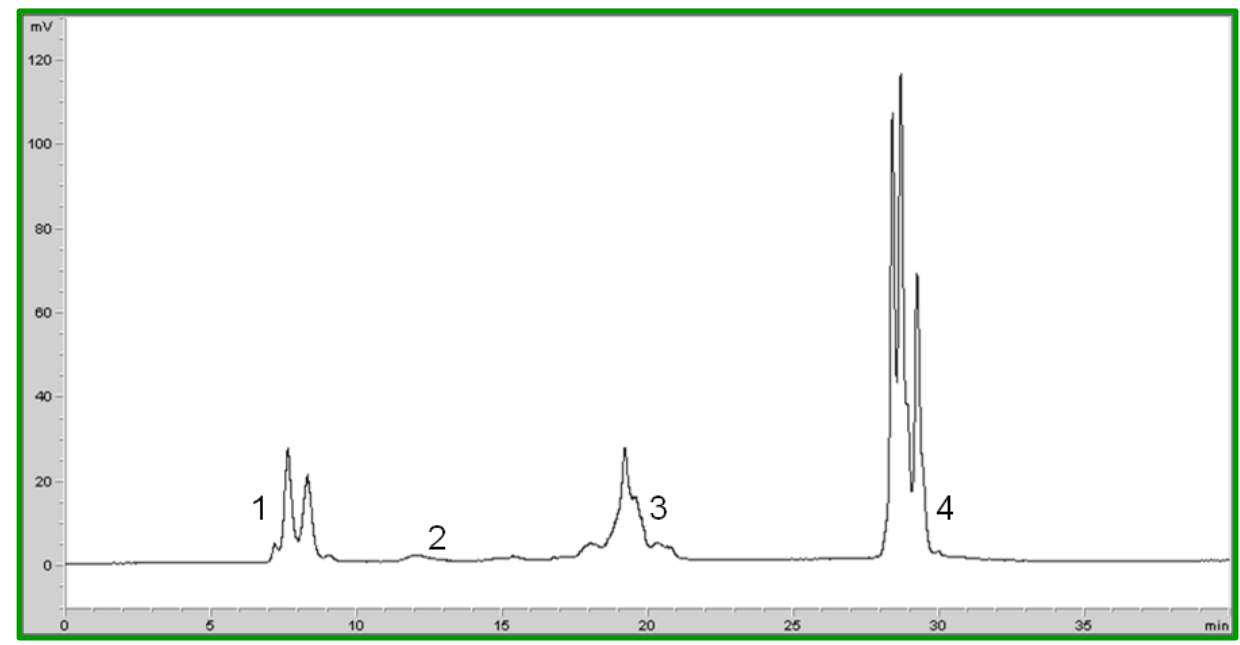

Figure B.2.HPLC-ELSD chromatogram with the neutral lipid classes, TAG (1), FFA (2), DAG (3) and MAG (4) detected in a glycerolysis reaction products sample.

\section{Quantification of neutral lipids}

The method has been calibrated with representatives of each group of compounds in working ranges depending on the lipid class. The calibration curves showed a good correlation according to the exponential relationship since the ELSD-signal does not linearly increase with substance concentration. Although ELSD parameters were optimized in order to enhance the sensitivity for all lipid representatives, the sensitivity of the detector varies depending on lipid class and within the same group; sensitivity of different compounds is also different (Schaefer et al. 2003).

The quantitative analysis of neutral lipids of interest (MAG, DAG, FFA and TAG) was performed by external calibration of a mixture of compounds of each lipid group. 1monopalmitin, 1-monoolein, 2-monoolein and 1-monodocosahexaenoin were used to calibrate MAG; dipalmitin and diolein for DAG calibration; and oleic and palmitic acids for FFA calibration. In case of TAG, a mix of pure standards (triolein, tripalmitin and trimiristin) showed an ELSD-signal quite different compared to the sardine oil, as a complex mixture 
of TAG; therefore, the refined sardine oil itself ( $\geq 99 \%$ of TAG) was used for the calibration of TAG. Table B.4 shows the calibration curves used in this work to quantify the neutral lipids in reaction products samples.

Table B.4. Calibration curves used in neutral lipid analysis by HPLC-ELSD

\begin{tabular}{cc} 
Lipid class & Calibration curve \\
TAG & log area $=1.8427+1.8494 *(\log \mu$ injected $)$ \\
FFA & log area $=2.1581+1.3377 *(\log \mu \mathrm{g}$ injected $)$ \\
DAG & $\log$ area $=2.8563+1.1330 *(\log \mu \mathrm{g}$ injected $)$ \\
MAG & $\log$ area $=2.5272+1.5326 *(\log \mu \mathrm{g}$ injected $)$ \\
\hline
\end{tabular}

The amount of each neutral lipid in the oil sample, expressed as \% wt. of the related lipid class, was determined by interpolation of the relative calibration curve. 


\section{Peroxide Value (PV)}

Peroxide value is a measure of the hydroperoxides compounds generated during the early stages of the oil oxidation process. They can be determined indirectly by redox titration in non-aqueous media. The peroxide group reacts with an excess of iodide ion (I') producing iodine, $\mathrm{I}_{2}$. The $\mathrm{I}_{2}$ generated was measured by titration with $\mathrm{Na}_{2} \mathrm{~S}_{2} \mathrm{O}_{3}$ solution. This method is based on the AOAC Method (AOAC Official Method 965.33 2000).

\section{Equipment}

An automatic titrator (Metrohm 905 Tritrando) equipped with a platinum electrode was used in this work. The method was developed in Tiamo software by S. Rebolleda (Rebolleda et al. 2012).

Reagents

- Chloroform $\left(\mathrm{CHCl}_{3}\right)$

- $\quad$ Glacial acetic acid $\left(\mathrm{CH}_{3} \mathrm{COOH}\right)$

- $\quad$ Freshly prepared saturated aqueous solution of potassium iodine (KI)

- $\quad$ Sodium thiosulphate solution $0.01 \mathrm{~N}$ in water $\left(\mathrm{Na}_{2} \mathrm{~S}_{2} \mathrm{O}_{3}\right)$

- Potasium iodate $\left(\mathrm{KIO}_{3}\right)$ dried at 180 o $\mathrm{C}$ overnight and cooled down during $2 \mathrm{~h}$

- $\mathrm{H}_{2} \mathrm{SO}_{4} 25 \%$ solution

\section{Experiment procedure}

\section{- Standarization of sodium thiosulphate 0.01Nsolution}

The standardization of sodium thiosulphate solution is necessary to obtain the real normality of the solution just prepared. It is made by triplicate. The scheme of the standardization process is represented in Figure B.3.

The sodium thiosulphate 0.01 solution factor $(f)$ was calculated according to Equation B.2:

$$
f=\frac{m_{K I O 3}}{V_{N a 2 S 203} \times 0.3567}
$$


where $\mathrm{m} \mathrm{KIO}_{3}$ is the mass in $\mathrm{mg}$ accurately weigh and $\mathrm{V}_{\mathrm{Na252O}}$ is the volume in $\mathrm{mL}$ needed in the titration.

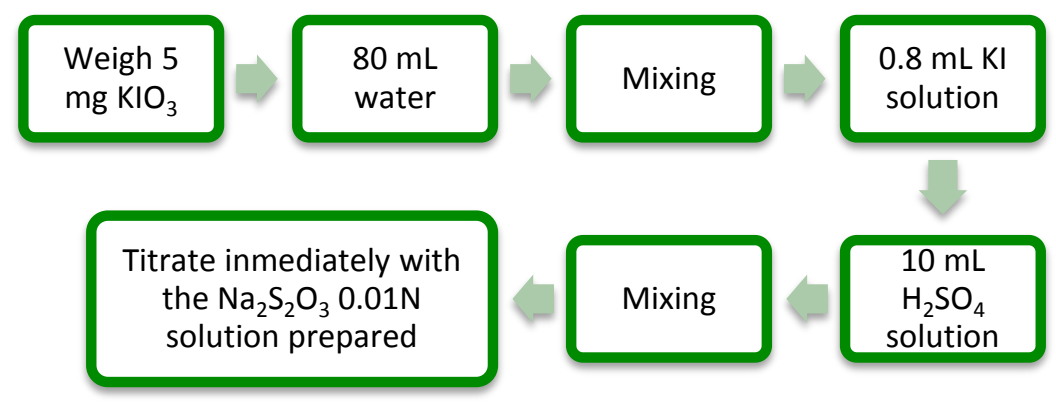

Figure B.3. Standarization of sodium thiosulphate 0.01 Nsolution for the analysis of peroxide values in oil samples.

\section{- $\quad$ Procedure to determine PV}

In this work the peroxide value (PV) was determined in sardine oil samples and in reaction products samples which contain a mix of TAG, DAG, MAG and FFA. The procedure was the same for both kinds of lipid samples and it is represented in Figure B.4. First blank titration was run according the following procedure but without sample. Low values for blanks are recommended (less than $0.05 \mathrm{~mL}$ ). Blank and sample were analyzed in triplicate.

\section{- Calculations}

The PV, expressed as milliequivalentsof $\mathrm{O}_{2}$ per $\mathrm{kg}$ of sample, was calculated according to Equation B.3:

$$
P V=\frac{\left(V_{s}-V_{b}\right) \times(N \times f)}{m} \times 1000
$$

where $\mathrm{V}_{\mathrm{s}}$ and $\mathrm{V}_{\mathrm{b}}$ are the necessary titrant volumes in $\mathrm{mL}$ of $\mathrm{Na}_{2} \mathrm{~S}_{2} \mathrm{O}_{3} 0.01 \mathrm{~N}$ solution for the sample and blank, respectively. $\mathrm{N}$ is the titrant normality $(0.01 \mathrm{~N}), f$ is the titrant solution 
factor calculated in the standardization of $\mathrm{Na}_{2} \mathrm{~S}_{2} \mathrm{O}_{3} 0.01 \mathrm{~N}$ solution and $\mathrm{m}$ is the weighed amount of sample in $\mathrm{g}$.

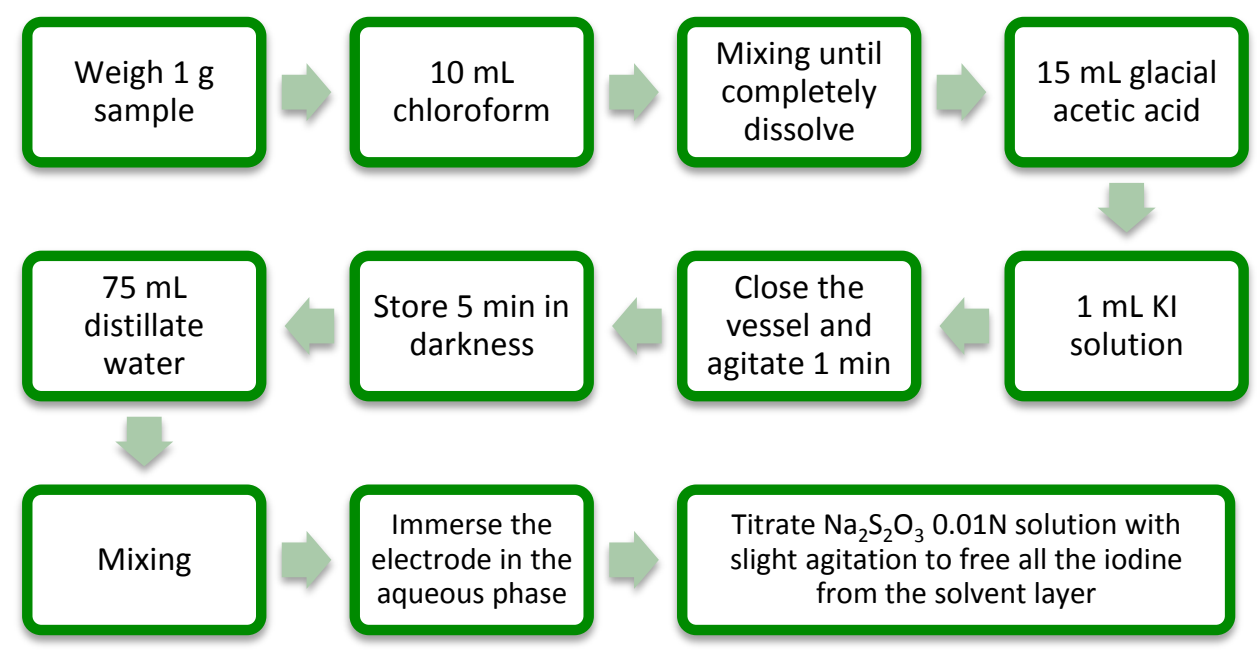

Figure B.4. Determination of peroxide value of lipid samples using the automatic titrator. 


\section{Anisidine Value (AV)}

The anisidine value is a measure of the non-volatile secondary oxidation products (mainly 2-alkenals and 2,4-dienals) present in oil samples at acidic conditions. The method is based on the reaction between $\mathrm{p}$-anisidine and aldehydic compounds producing yellowcolored compounds which are measured at $350 \mathrm{~nm}$. The AV was determined according to AOCS Method (AOCS Official Method Cd 18-90 2017).

\section{Equipment}

Absorbance measurements were carried out in a UV-Vis spectrophotometer (Hitachi, U2000 ) at $350 \mathrm{~nm}$ with glass cuvettes of $10 \mathrm{~mm}$.

Reagents

- Tert-pentanol

- Glacial acetic acid

- $\quad$ Freshly prepared $\mathrm{p}$-anisidine solution $(0.25 \% \mathrm{w} / \mathrm{v}$ in glacial acetic acid)

\section{Experimental procedure}

In this work anisidine value (AV) was determined in sardine oil samples and in reaction products samples which contain a mix of TAG, DAG, MAG and FFA. The procedure was the same for both kinds of lipid samples and it is represented in Figure B.5. Tert-pentanol was used to dissolve the sample instead of hexane or isooctane due to the poor solubility of the reaction products in these solvents. The presence of moisture in any of the reagents or in the test sample leads to incomplete reaction and, consequently, low values.

Blank was done in each analysis. Samples were analyzed in triplicate.

\section{- Calculations}

The AV was calculated according to Equation B.4:

$$
A V=\frac{25 \times\left(1.2 \times A_{2}-A_{1}\right)}{m}
$$


where $A_{2}$ is the absorbance of the lipid sample solution after reaction with the anisidine solution, $A_{1}$ is the absorbance of the lipid sample solution and $m$ is the weight of lipid sample in g.

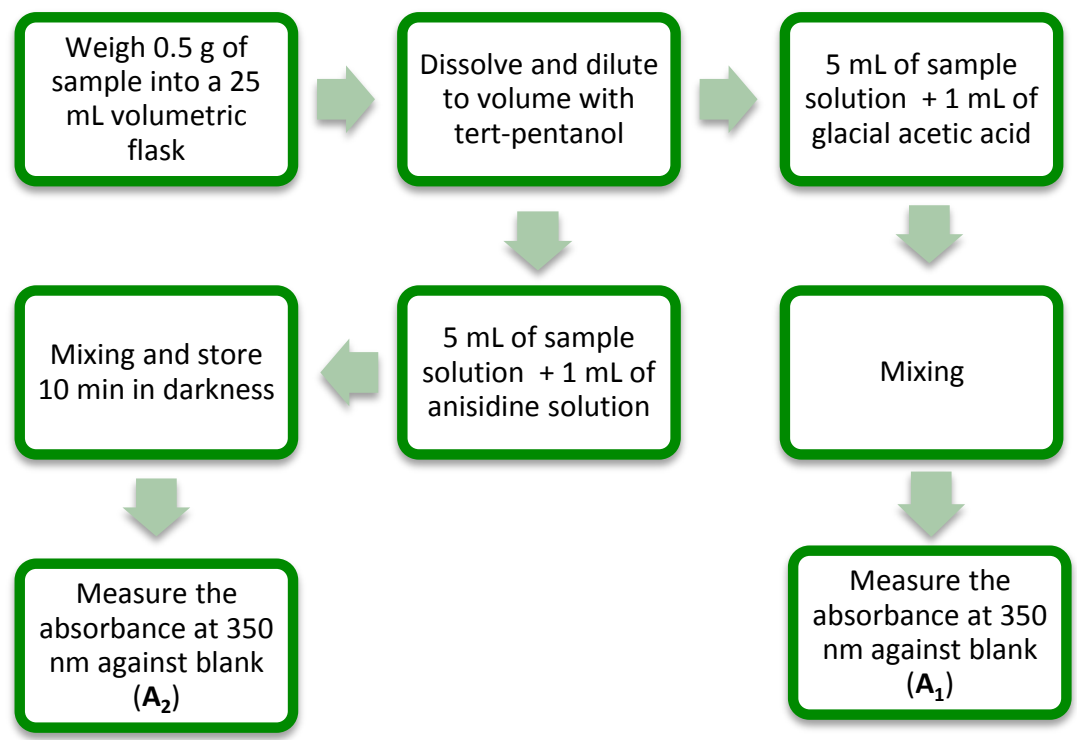

Figure B.5. Determination of anisidine value of lipid samples of this work. 


\section{Thiobarbituric Acid Reactive Substances (TBARS)}

TBARS value is related to those secondary oxidation products that react with thiobarbituric acid (TBA) andform a pink complex with strong absorbance at $538 \mathrm{~nm}$. These TBARS are generally referred to malondialdehyde (MDA), one of the most important products of oxidation from decomposition of unsaturated fatty acid. TBARS values were determined according to the spectrophotometric method described by Norveel Semb (Norveel Semb 2012).

\section{Equipment}

Absorbance measurements were carried out in a UV-Vis spectrophotometer (Hitachi, U2000) at $538 \mathrm{~nm}$ with glass cuvettes of $10 \mathrm{~mm}$.

Water bath and centrifuge were also required for heating and centrifuging samples.

\section{Reagents}

- $\quad$ Glacial acetic acid

- Chloroform

- $\quad 0.04$ M 2-thiobarbituric acid (TBA) stock solution* in glacial acetic acid

TBA work solution: prepare max. 30 min before analysis by mixingTBA stock solution*, chloroform and sodium sulphite solution at volume ratios of 12-8-1, respectively (alternative ratios: 36-24-3, 60-40-5).

- $\quad 0.3 \mathrm{M}$ sodium sulphite $\left(\mathrm{Na}_{2} \mathrm{SO}_{3}\right)$ solution in water

- $\quad 0.28 \mathrm{M}$ trichloracetic acid (TCA) solution in water

- $\quad 0.1 \mathrm{mM} \mathrm{1,1,3,3,-tetraethoxypropane} \mathrm{(TEP)} \mathrm{work} \mathrm{solution} \mathrm{in} \mathrm{water.}$

\section{Experimental procedure}

In this work TBARS values were determined in sardine oil samples. The procedure was represented in Figure B.6. Blank was prepared in the same way, but without the oil. Samples were analyzed in triplicate. 


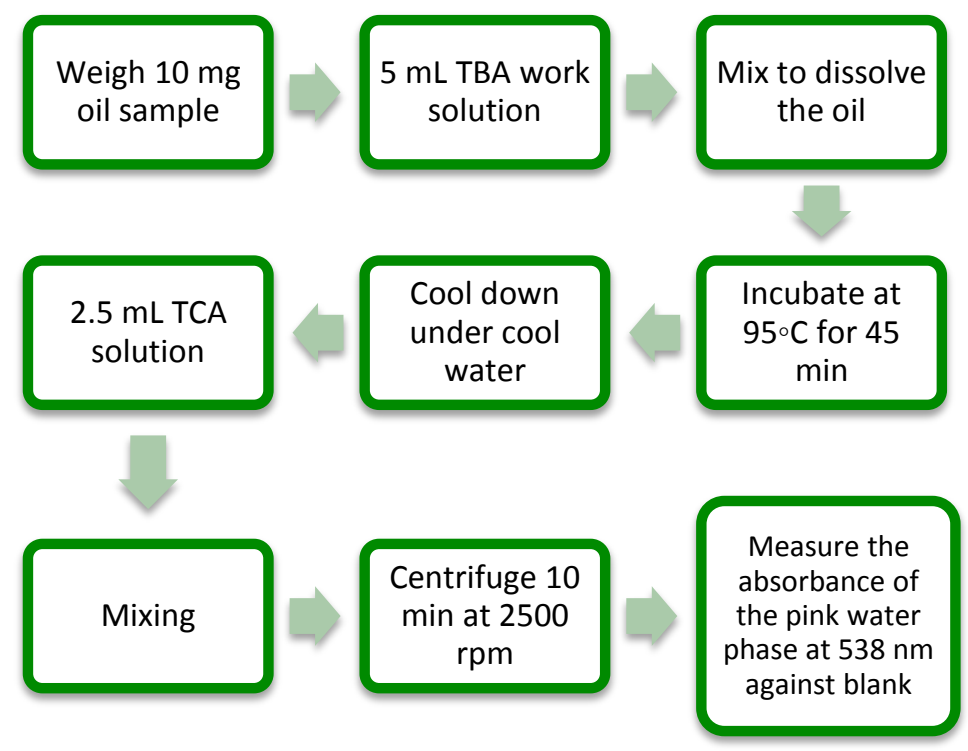

Figure B.6. Determination of TBARS value of oil samples of this work.

- Standard curve

Standard curve is based on $0.1 \mathrm{mM}$ 1,1,3,3,-tetraethoxypropane (TEP) work solution. Although TBARS is usually expressed as mg of malondialdehyde (MDA) per kilogram of oil, TEP is used as the MDA standard. MDA is a highly unstable compound and is unavailable as a standard. TEP hydrolyzes under mild acid conditions to yield MDA. Therefore TEP can be used as a source of MDA and as a standard for the quantitative determination of TBARS.

The calibration was carried out following the same procedure as sample analysis (Figure B.6), only the oil is replaced by TEP work solution diluted according to Table B.5. Each TEP concentration should be duplicated. Figure B.7 represents the standard curve obtained experimentally in this work. 
Table B.5. TEP dilutions to make the standard curve in this work

$\mu \mathrm{L}$ TEP work solution $\quad \mu \mathrm{L} \mathrm{H}_{2} \mathrm{O} \quad \mathrm{nmol}$ TEP

\begin{tabular}{ccc}
0 & 200 & 0 \\
25 & 150 & 2.5 \\
50 & 100 & 5 \\
100 & 50 & 10 \\
150 & 25 & 15 \\
200 & 0 & 20 \\
\hline
\end{tabular}

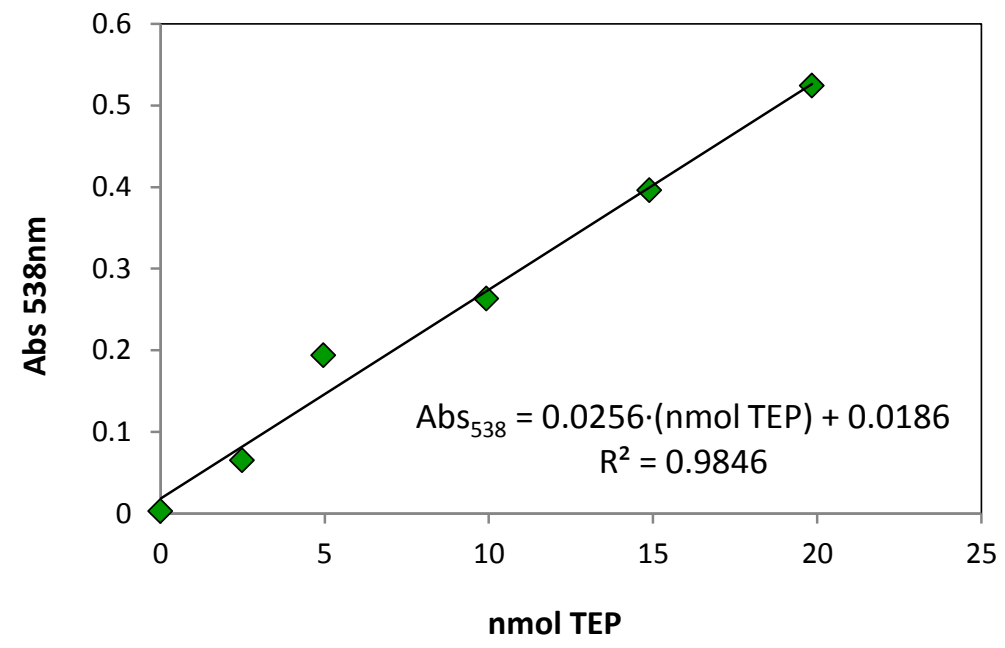

Figure B.7. Standard curve used in this work for TBARS determination.

Since in the reaction nmol TEP yields to nmol of MDA, the amount of TBARS in oil samples, expressed as mg of MDA/Kg of oil, can be calculated according to Equation B.5: 
$\operatorname{TBARS}(\mathrm{mg} M D A / K g$ oil $)=\frac{(A s-b) \times 72}{a \times m \times 1000}$

where As is the absorbance of the oil sample against blank, $a$ is the slope of the standard curve (0.0256 in this work), $\mathrm{b}$ is the intercept of the standard curve (0.0186 in this work), $\mathrm{m}$ is the amount of sample in g., 72 is de molecular weight of MDA and 1000 is the corresponding factor to converts $\mathrm{nmol} / \mathrm{g}$ to $\mathrm{mmol} / \mathrm{kg}$. 
REFERENCES 



\section{REFERENCES}

Americas V. 2012. http://www.ohmartvega.com/en/dielectric constants.htm.

AMR. 2010. Krill oil monograph. Altern. Med. Rev. 15, 84-86.

Ando Y, Narukawa K. 2002. Regiospecific distribution of highly unsaturated fatty acids in triacylglycerols of Artemia nauplii enriched with marine oils. J. Oleo Sci. 51, 615-620.

AOAC Official Method 965.33. 2000. Peroxide Value of Oils and Fats.

AOAC Official Method 991.39. 2000. Fatty Acids in Encapsulated Fish Oils and Fish Oil Methyl and Ethyl Esters.

AOCS Official Method Ca 5a-40. 2009. Free Fatty Acids.

AOCS Official Method Cd 18-90. 2017. p-Anisidine Value.

Awadallak JA et al. 2013. Ultrasonics Sonochemistry Enzymatic catalyzed palm oil hydrolysis under ultrasound irradiation: Diacylglycerol synthesis. Ultrason. Sonochemistry 20, 1002-1007.

Bailey JE. 1986. Biochemical Engineering Fundamentals, McGraw-Hill, New York.

Bandarra NM et al. 1997. Seasonal Changes in Lipid Composition of Sardine (Sardina pilchardus). J. Food Sci. 62.

Barba FJ, Orlien V. 2017. Processing, bioaccessibility and bioavailability of bioactive sulfur compounds: Facts and gaps. J. Food Compos. Anal. 61, 1-3.

Berger R, McPherson W. 1979. Fractional distillation. J. Am. Oil Chem. Soc. 56, 8-9.

Blanco $\mathrm{M}$ et al. 2007. Towards sustainable and efficient use of fishery resources: present and future trends. Trends Food Sci. Technol. 18, 29-36.

Borch-Jensen C, Mollerup J. 1997. Phase equilibria of fish oil in sub-and supercritical 
carbon dioxide. Fluid Phase Equilib. 138, 179-21.

Bornscheuer UT. 1995. Lipase-catalyzed syntheses of monoacylglycerols. Enzyme Microb. Technol. 17, 578-586.

Bosch AC et al. 2016. Heavy metals in marine fish meat and consumer health: A review. J. Sci. Food Agric. 96, 32-48.

Brady C et al. 1988. Lipase immobilized on a hydrophobic, microporous support for the hydrolysis of fats. J. Am. Oil Chem. Soc. 65, 1988.

Breivik H, Haraldsson GG, Krisfinsson G. 1997. Preparation of Highly Purified Concentrates of Eicosapentaenoic Acid and Docosahexaenoic Acid. J. Am. Oil Chem. Soc. 74, 14251429.

Brown JB, Kolb DK. 1955. Applications of low temperature crystallization in the separation of the fatty acids and their compounds. Prog. Chem. Fats Other Lipids 3, 57-72.

Bucio SL et al. 2015. Kinetic Study for the Ethanolysis of Fish Oil Catalyzed by Lipozyme ${ }^{\circledR}$ 435 in Different Reaction Media. J. Oleo Sci. 64, 431-441.

Bucio SL et al. 2013. Liquid-liquid equilibrium for ethanolysis systems of fish oil. J. Chem. Eng. Data 58, 3118-3124.

Bucio SL et al. 2016. Study of the influence of process parameters on liquid and supercritical $\mathrm{CO} 2$ extraction of oil from rendered materials: Fish meal and oil characterization. J. Supercrit. Fluids 107, 270-277.

Cai C et al. 2016. Immobilization of Candida antarctica lipase B onto SBA-15 and their application in glycerolysis for diacylglycerols synthesis. Food Chem. 212, 205-212.

Calder PC. 2006. n-3 Polyunsaturated fatty acids, inflammation, and inflammatory diseases. Am. J. Clin. Nutr. 83, 1505-1519.

Canas BJ, Yurawecz MP. 1999. Ethyl Carbamate Formation During Urea Complexation for 
Fractionation of Fatty Acids. J. Am. Oil Chem. Soc. 76, 537.

Carvalho CML, Cabral JMS. 2000. Reverse micelles as reaction media for lipases. Biochimie 82, 1063-1085.

Castillo E et al. 1994. Polar substrates for enzymatic reactions in supercritical CO2: How to overcome the solubility limitation. Biotechnol. Lett. 16, 169-174.

Cheirsilp B. 2007. A mathematical model approach to a glycerolysis reaction for monoacylglycerol production. WIT Trans. Modelling Simul. 46, 225-232.

Cheirsilp B, Kaewthong W, H-Kittikun A. 2007. Kinetic study of glycerolysis of palm olein for monoacylglycerol production by immobilized lipase. Biochem. Eng. J. 35, 71-80.

Chen B et al. 2008. Candida antarctica Lipase B Chemically Immobilized on EpoxyActivated Micro- and Nanobeads: Catalysts for Polyester Synthesis. Biomacromolecules 9, 463-471.

Chesterfield DM et al. 2012. Production of biodiesel via ethanolysis of waste cooking oil using immobilised lipase. Chem. Eng. J. 207-208, 701-710.

Chowdhury FI, Saleh MA. 2014. Viscosities and deviations in viscosity of tert-butanol with n-butylamine, di-n-butylamine and tri-n-butylamine. J. Mol. Liq. 191, 156-160.

Ciriminna R et al. 2017. Enhancing and improving the extraction of omega-3 from fish oil. Sustain. Chem. Pharm. 5, 54-59.

Cruz-Hernandez C et al. 2012. Benefits of structured and free monoacylglycerols to deliver eicosapentaenoic (EPA) in a model of lipid malabsorption. Nutrients 4, 1781-1793.

Damstrup ML, Abildskov J, et al. 2006. Evaluation of binary solvent mixtures for efficient monoacylglycerol production by continuous enzymatic glycerolysis. J. Agric. Food Chem. 54, 7113-7119.

Damstrup ML, Jensen T, et al. 2006. Production of heat-sensitive monoacylglycerols by 
enzymatic glycerolysis in tert-pentanol: Process optimization by response surface methodology. J. Am. Oil Chem. Soc. 83, 27-33.

Damstrup ML et al. 2005. Solvent Optimization for Efficient Enzymatic Monoacylglycerol Production Based on a Glycerolysis Reaction. J. Am. Oil Chem. Soc. 82, 559-564.

Davatgaran-Taghipour $Y$ et al. 2017. Polyphenol nanoformulations for cancer therapy : experimental evidence and clinical perspective. Int. J. Nanomedicine 12, 2689-2702.

Davies JT. 1957. A quantitative kinetic theory of emulsion type. I. Physical chemistry of the emulsion agent. in Proceedings of 2nd International Congress Surface Activity, London 1957. pp. 426-438.

Dawczynski C, Schubert R, Jahreis G. 2007. Amino acids, fatty acids, and dietary fibre in edible seaweed products. Food Chem. 103, 891-899.

De Deckere EAM et al. 1998. Health aspects of fish and n-3 polyunsaturated fatty acids from plant and marine origin. Eur. J. Clin. Nutr. 52, 749-753.

DeFilippis AP, Sperling LS. 2006. Understanding omega-3's. Am. Heart J. 151, 564-570.

Derawi D, Afiqah Zairul N, Fadlly Jumadi M. 2017. Preliminary Study on Production of Monoacylglycerol and Diacylglycerol of Virgin Coconut Oil Via Enzymatic Glycerolysis Using Lipase Candida antarctica (Novozyme 435). Malaysian J. Anal. Sci. 21, 37-45.

Devi BLAP et al. 2008. Enzymatic synthesis of designer lipids. OCL - Ol. Corps Gras Lipides 15, 189-195.

Diaz TG et al. 2005. Characterization of virgin olive oils according to its triglycerides and sterols composition by chemometric methods. Food Control 16, 339-347.

Van Dijk M et al. 2012. Fish Oil Production, Consumption and Health Benefits, Nova Science Publishers, Inc.

Ding $\mathrm{N}$ et al. 2013. Short-term Effects of Different Fish Oil Formulations on Tissue 
Absorption of Docosahexaenoic Acid in Mice Fed High-and Low-Fat Diets. J. Oleo Sci. 62, 883-891.

Domanska U. 1998. Thermodynamics of branched alcohols II. Solid-liquid equilibria for systems containing tert-butanol and long-chain n-alkanes. Experimental results and comparison with DISQUAC predictions. Fluid Phase Equilib. 147, 251-270.

Domingo JL et al. 2007. Benefits and risks of fish consumption. Part I. A quantitative analysis of the intake of omega-3 fatty acids and chemical contaminants. Toxicology 230, 219-226.

Dong HP, Wang YJ, Zheng YG. 2010. Enantioselective hydrolysis of diethyl 3hydroxyglutarate to ethyl (S)-3-hydroxyglutarate by immobilized Candida antarctica lipase B. J. Mol. Catal. B Enzym. 66, 90-94.

Dyerberg J et al. 2010. Bioavailability of marine n-3 fatty acid formulations. Prostaglandins Leukot. Essent. Fat. Acids 83, 137-141.

Esmelindro ÂFA et al. 2008. Lipase-catalyzed production of monoglycerides in compressed propane and AOT surfactant. J. Supercrit. Fluids.

European Food Safety Authority. 2010. Scientific opinion on dietary reference values for fats, including saturated fatty acids, polyunsaturated fatty acids, monounsaturated fatty acids, trans fatty acids, and cholesterol. EFSA J. 8, 1461.

European Pharmacopoeia 5.0. 2005. Omega-3-acid Triglycerides. 6, 2144-2146.

FAO/WHO. 2008. Interim Summary of Conclusions and Dietary Recommendations on Total Fat \& Fatty Acids. FAO/WHO Expert Consult., 1-14.

Feltes MMC et al. 2010. Assessment of process parameters on the production of diglycerides rich in omega-3 fatty acids through the enzymatic glycerolysis of fish oil. Eur. Food Res. Technol. 231, 701-710.

Feltes MMC et al. 2012. Evaluation of the use of surfactants as additives in enzymatic 
glycerolysis reactions. Eur. J. Lipid Sci. Technol. 114, 1352-1357.

Feltes MMC et al. 2013. The Production, Benefits, and Applications of Monoacylglycerols and Diacylglycerols of Nutritional Interest. Food Bioprocess Technol. 6, 17-35.

Fiametti KG et al. 2009. Kinetics of Solvent-Free Lipase-Catalyzed Production of Monoacylglycerols from Olive Oil in Aerosol-OT Surfactant. Ind. Eng. Chem. Res. 48, 708-712.

Fiametti KG et al. 2012. Kinetics of ultrasound-assisted lipase-catalyzed glycerolysis of olive oil in solvent-free system. Ultrason. Sonochem. 19, 440-451.

Fiori L et al. 2017. From Fish Waste to Omega-3 Concentrates in a Biorefinery Concept. Waste and Biomass Valorization 0, 0.

Fogler HS. 1999. Elements of Chemical Reactions Engineering, Prentice-Hall International Inc., New Jersey.

Fortin S. 2012.Compositions Comprising Polyunsaturated Fatty Acid Monoglycerides, Derivatives Thereof and Uses Thereof. US 2012/0213823 A1.

França BB et al. 2009. Liquid-liquid equilibria for castor oil biodiesel + glycerol + alcohol. J. Chem. Eng. Data 54, 2359-2364.

Frankel EN. 1984. Lipid Oxidation: Mechanisms, Products and Biological Significance. J. Am. Oil Chem. Soc. 61, 1908-1917.

Fregolente LV et al. 2007. Effect of operating conditions on the concentration of monoglycerides using molecular distillation. Chem. Eng. Res. Des. 85, 1524-1528.

Fregolente LV et al. 2006. Optimization of Distilled Monoglycerides Production. Appl. Biochem. Biotechnol. 129, 680-693.

Fregolente PBL et al. 2010. Monoglyceride and diglyceride production through lipasecatalyzed glycerolysis and molecular distillation. Appl. Biochem. Biotechnol. 160, 
1879-1887.

Fureby AM et al. 1996. Acyl group migrations in 2-monoolein. Biocatal. Biotransformation 14, 89-111.

Gámez-Meza N et al. 2003. Concentration of eicosapentaenoic acid and docosahexaenoic acid from fish oil by hydrolysis and urea complexation. Food Res. Int. 36, 721-727.

García-Moreno PJ et al. 2013. Optimization of bleaching conditions for sardine oil. J. Food Eng. 116, 606-612.

GOED. 2015. Voluntary Monograph Version 5 (Issue Date November 19).

Gomes de Oliveira C, Marques Porto L. 2005. A Kinetic Model for Bleaching Vegetable Oils. J. Am. Oil Chem. Soc. 82, 537-542.

Gómez-Alonso S, Mancebo-Campos V, Salvador MD. 2004. Oxidation kinetics in olive oil triacylglycerols under accelerated shelf-life testing $\left(25-75^{\circ} \mathrm{C}\right)$. Eur. J. Lipid Sci. Technol. 106, 369-375.

Gonçalves CB, Batista E, Meirelles AJA. 2002. Liquid-Liquid Equilibrium Data for the System Corn Oil + Oleic Acid + Ethanol + Water at 298.15 K. J. Chem. Eng. Data 47, 416-420.

González JA et al. 1996. Thermodynamics of branched alcohols I. Extension of DISQUAC to tert-alcohols-n-alkanes or tert-alcohols-cyclohexane mixtures. Fluid Phase Equilib. 119, 81-96.

Gotoh N et al. 2006. Quantification Method for Triglyceride Molecular Species in Fish Oil with High Performance Liquid Chromatography - Ultraviolet Detector - Evaporative Light Scattering Detector. J. Oleo Sci. 55, 457-463.

Goyens PLL, Mensink RP. 2006. Effects of alpha-linolenic acid versus those of EPA/DHA on cardiovascular risk markers in healthy elderly subjects. Eur. J. Clin. Nutr. 60, 978984. 
Guillen MD, Cabo N. 2002. Fourier transform infrared spectra data versus peroxide and anisidine values to determine oxidative stability of edible oils. Food Chem. 77, 503510.

Guo Z, Xu X. 2006. Lipase-catalyzed glycerolysis of fats and oils in ionic liquids: a further study on the reaction system. Green Chem. 8, 54-62.

Haraldsson GG, Gudmundsson BÖ, Almarsson Ö. 1995. The synthesis of homogeneous triglycerides of eicosapentaenoic acid and docosahexaenoic acid by lipase. Tetrahedron 51, 941-952.

Harris WS. 2007. Omega-3 fatty acids and cardiovascular disease: A case for omega-3 index as a new risk factor. Pharmacol. Res. 55, 217-223.

Helfferich FG. 1962. Ion Exchange, McGraw-Hill, New York.

Hernandez EM. 2014. Issues in fortification and analysis of omega-3 fatty acids in foods. Lipid Technol. 26, 103-106.

Hierro MTG et al. 1992. Determination of the triglyceride composition of avocado oil by high-performance liquid chromatography using a light-scattering detector. J. Chromatogr. A 607, 329-338.

Homayooni B, Sahari MA, Barzegar M. 2014. Concentrations of omega-3 fatty acids from rainbow sardine fish oil by various methods. Int. Food Res. J. 21, 743-748.

Hoyland D, Taylor A. 1991. A Review of the Methodology of the 2-Thiobarbituric Acid Test. Food Chem. 40, 271-291.

Jayasinghe C, Gotoh N, Wada S. 2012. Regiospecific analysis of shark liver triacylglycerols. J. Am. Oil Chem. Soc. 89, 1873-1884.

Jin J et al. 2011. Production of diacylglycerols from glycerol monooleate and ethyl oleate through free and immobilized lipase-catalyzed consecutive reactions. N. Biotechnol. 28, 190-195. 
Kadir Topuz O, Yerlikaya P, Uçak İ. 2015. Influence of pomegranate peel ( Punica granatum ) extract on lipid oxidation in anchovy fish oil under heat accelerated conditions. $J$. Food Sci. Technol. 52, 625-632.

Kahveci D, Xu X. 2011. Repeated hydrolysis process is effective for enrichment of omega 3 polyunsaturated fatty acids in salmon oil by Candida rugosa lipase. Food Chem. 129, $1552-1558$.

Kamal-Eldin A, Yanishlieva N. 2005. Chapter 10. Kinetic Analysis of Lipid Oxidation Data. in AOCS Lipid Library.

Kamal-Eldin A, Yanishlieva N V. 2002. N-3 fatty acids for human nutrition: Stability considerations. Eur. J. Lipid Sci. Technol. 104, 825-836.

Khaddaj-Mallat R et al. 2016. Novel n-3 PUFA monoacylglycerides of pharmacological and medicinal interest: Anti-inflammatory and anti-proliferative effects. Eur. J. Pharmacol. 792, 70-77.

Kim SK, Mendis E. 2006. Bioactive compounds from marine processing byproducts - A review. Food Res. Int. 39, 383-393.

Komprda T. 2012. Eicosapentaenoic and docosahexaenoic acids as inflammationmodulating and lipid homeostasis influencing nutraceuticals: A review. J. Funct. Foods 4, 25-38.

Kris-Etherton PM, Harris WS, Appel LJ. 2002. Fish consumption, fish oil, omega-3 fatty acids, and cardiovascular disease. Circulation 19, 2747-2757.

Kristensen JB, Xu X, Mu H. 2005. Diacylglycerol synthesis by enzymatic glycerolysis: Screening of commercially available lipases. JAOCS, J. Am. Oil Chem. Soc. 82, 329334.

Krüger RL et al. 2010. Improvement of mono and diacylglycerol production via enzymatic glycerolysis in tert-butanol system. Eur. J. Lipid Sci. Technol. 112, 921-927. 
Labuza T, Bergquist S. 1983. Kinetics of Oxidation of Potato Chips under Constant Temperature and Sine Wave Temperature Conditions. J. Food Process. Preserv. 48, $712-715$.

Lanza M et al. 2009. Liquid-liquid equilibrium data for systems containing vegetable oils, anhydrous ethanol, and hexane at $(313.15,318.15$, and 328.15) K. J. Chem. Eng. Data 54, 1850-1859.

Lanza M et al. 2008. Liquid-Liquid Equilibrium Data for Reactional Systems of Ethanolysis at 298.3 K. J. Chem. Eng. Data 53, 5-15.

Lawson LD, Hughes BG. 1988a. Absorption of eicosapentaenoic acid and DHA from fish oil triacylglycerols or fish oil ethyl esters co ingested with a high fat meal. Biochem. Biophys. Res. Commun. 156, 960-963.

Lawson LD, Hughes BG. 1988b. Human absorption of fish oil fatty acids as triacylglycerols, free acids, or ethyl esters. Biochem. Biophys. Res. Commun. 152, 328-335.

Lee J, Lee Y, Choe E. 2007. Temperature dependence of the autoxidation and antioxidants of soybean, sunflower, and olive oil. Eur. Food Res. Technol. 226, 239-246.

Lee TW, Hastilow Cl. 1999. Quantitative Determination of Triacylglycerol Profile of Structured Lipid by Capillary Supercritical Fluid Chromatography and HighTemperature Gas Chromatography. J. Am. Oil Chem. Soc. 76, 1405-1413.

Li J et al. 2016. The role of Omega-3 docosapentaenoic acid in pregnancy and early development. Eur. J. Lipid Sci. Technol. 118, 1692-1701.

Liang J-H, Hwang LS. 2000. Fractionation-of-squid-visceral-oil-ethyl-esters-by-short-pathdistillation. J. Am. Oil Chem. Soc. 77, 773-777.

Lin TJ, Chen SW. 2008. Enrichment of $n-3$ polyunsaturated fatty acids into acylglycerols of borage oil via lipase-catalyzed reactions under supercritical conditions. Chem. Eng. J. $141,318-326$. 
Lin TJ, Chen SW, Chang AC. 2006. Enrichment of $n-3$ PUFA contents on triglycerides of fish oil by lipase-catalyzed trans-esterification under supercritical conditions. Biochem. Eng. J. 29, 27-34.

López-Hernández A et al. 2004. Method for analysis of TAG formed by reaction of fish oil with hydrogenated soybean oil. J. Am. Oil Chem. Soc. 81, 743-747.

Lyberg AM, Adlercreutz P. 2008. Lipase-catalysed enrichment of DHA and EPA in acylglycerols resulting from squid oil ethanolysis. Eur. J. Lipid Sci. Technol. 110, 317324.

Maduro RM, Aznar M. 2008. Liquid-liquid equilibrium of ternary systems 1-butyl-3methylimidazolium hexafluorophosphate + aromatic + aliphatic. Fluid Phase Equilib. 265, 129-138.

Majid N, Cheirsilp B. 2012. Optimal conditions for the production of monoacylglycerol from crude palm oil by an enzymatic glycerolysis reaction and recovery of carotenoids from the reaction product. Int. J. Food Sci. Technol. 47, 793-800.

Martín D et al. 2012. Oxidative stabilization of ultra-high omega-3 concentrates as ethyl esters or triacylglycerols. Food Res. Int. 45, 336-341.

Martín Valverde L et al. 2012. Concentration of docosahexaenoic acid (DHA) by selective alcoholysis catalyzed by lipases. J. Am. Oil Chem. Soc. 89, 1633-1645.

Martín Valverde L et al. 2013. Concentration of eicosapentaenoic acid (EPA) by selective alcoholysis catalyzed by lipases. Eur. J. Lipid Sci. Technol. 115, 990-1004.

Masson S L. 1994. Control de Calidad de Insumos y Dietas Acuicolas. http://www.fao.org/docrep/field/003/ab482s/ab482s10.htm.

Mataix J, Gil Á. 2005. Libro Blanco de los Omega-3. Los ácidos grasos poliinsaturados Omega 3 y monoinsaturados tipo oleico y su papel en la salud, Puleva Food.

Matsuda T. 2013. Recent progress in biocatalysis using supercritical carbon dioxide. J. 
Biosci. Bioeng. 115, 233-241.

McNeill GP, Yamane T. 1991. Further Improvements in the Yield of Monoglycerides During Enzymatic Glycerolysis of Fats and Oils. J. Am. Oil Chem. Soc. 68, 6-10.

Medina-Gonzalez $Y$ et al. 2013. Phase equilibrium of the CO2/glycerol system: Experimental data by in situ FT-IR spectroscopy and thermodynamic modeling. $J$. Supercrit. Fluids 73, 97-107.

De Meester F, Watson RR, Zibadi S. 2013. Omega-6/3 Fatty Acids Functions, Sustainability Strategies and Perspectives, Humana Press.

Melgosa R et al. 2017. Supercritical carbon dioxide as solvent in the lipase-catalyzed ethanolysis of fi sh oil : Kinetic study. J. CO2 Util. 17, 170-179.

Mesquita FMR et al. 2012. Liquid-liquid equilibria of systems containing cottonseed biodiesel+glycerol+ethanol at 293.15, 313.15 and 333.15K. Fluid Phase Equilib. 318, $51-55$.

Mishra VK, Temelli F, Ooraikul B. 1993. Extraction and purification of w-3 fatty acids with an emphasis on supercritical fluid-A review. Food Res. Int. 26, 217-226.

Mize HE et al. 2013. Emulsions of Crude Glycerin from Biodiesel Processing with Fuel Oil for Industrial Heating. J. Agric. Food Chem. 61, 1319-1327.

Monte ML et al. 2015. Bleaching with blends of bleaching earth and activated carbon reduces color and oxidation products of carp oil. Eur. J. Lipid Sci. Technol. 117, 829836.

Monte Blanco SFM et al. 2015. Optimization of diacylglycerol production by glycerolysis of fish oil catalyzed by Lipozyme TL IM with Tween 65. Bioprocess Biosyst. Eng. 38, 2379-2388.

Moquin PHL et al. 2005. Kinetic modeling of the glycerolysis reaction for soybean oils in supercritical carbon dioxide media. J. Am. Oil Chem. Soc. 82, 613-617. 
Moreda W, Pérez-Camino MC, Cert A. 2003. Improved method for the determination of triacylglycerols in olive oils by high performance liquid chromatography. Grasas y Aceites 54, 175-179.

Morin C et al. 2014. Docosapentaenoic acid monoacylglyceride reduces inflammation and vascular remodeling in experimental pulmonary hypertension. AJP Hear. Circ. Physiol. 307, H574-H586.

Morin C, Rousseau E, et al. 2015. Effect of docosahexaenoic acid monoacylglyceride on systemic hypertension and cardiovascular dysfunction. Am. J. Physiol. Heart Circ. Physiol. 309, H93-H102.

Morin C, Fortin S, et al. 2013. MAG-EPA resolves lung inflammation in an allergic model of asthma. Clin. Exp. Allergy 43, 1071-1082.

Morin C, Blier PU, Fortin S. 2015. Eicosapentaenoic acid and docosapentaenoic acid monoglycerides are more potent than docosahexaenoic acid monoglyceride to resolve inflammation in a rheumatoid arthritis model. Arthritis Res. Ther. 17, 142.

Morin C, Blier PU, Fortin S. 2016. MAG-EPA reduces severity of DSS-induced colitis in rats. Am. J. Physiol. Gastrointest. Liver Physiol. 310, G808-21.

Morin C, Rousseau É, Fortin S. 2013. Anti-proliferative effects of a new docosapentaenoic acid monoacylglyceride in colorectal carcinoma cells. Prostaglandins Leukot. Essent. Fat. Acids.

Moyad MA. 2005. An introduction to dietary/supplemental omega-3 fatty acids for general health and prevention: Part I. Urol. Oncol. Semin. Orig. Investig. 23, 28-35.

Muñío M del M et al. 2008. Synthesis of 2-monoacylglycerols rich in polyunsaturated fatty acids by ethanolysis of fish oil catalyzed by 1,3 specific lipases. Process Biochem. 43, 1033-1039.

Najera Al et al. 1998. Determination of triacylglycerol composition of Idiazabal cheese. Chromatographia 47, 579-586. 
Nichols PD et al. 2014. Recent advances in omega-3: Health benefits, Sources, Products and bioavailability. Nutrients 6, 3727-3733.

Noriega-Rodríguez JA et al. 2009. Oil production from sardine ( Sardinops sagax caerulea ) Producción de aceite a partir de sardina ( Sardinops sagax caerulea). CyTA -Journal Food 7, 173-179.

Norveel Semb T. 2012. Analytical Methods for Determination of the Oxidative Status in Oils (Master's Thesis; NTNU).

Nunes AVM et al. 2013. Solubility of CO2 in glycerol at high pressures. Fluid Phase Equilib. 358, 105-107.

O’Brien RD. 2004. Fats and Oils. Formulating and Processing for Applications Second Ed., CRC Press LLC.

Okada T, Morrisset M. 2007. Recovery and Characterization of Sardine Oil Extracted by pH Adjustment. J. Agric. Food Chem 55, 1808-1813.

Olivares-Carrillo $\mathrm{P}$ et al. 2014. Estimation of critical properties of reaction mixtures obtained in different reaction conditions during the synthesis of biodiesel with supercritical methanol from soybean oil. Chem. Eng. J., 418-432.

Oliveira ACM, Miller MR. 2014. Purification of Alaskan walleye pollock (Gadus chalcogrammus) and New Zealand hoki (Macruronus novaezelandiae) liver oil using short path distillation. Nutrients 6, 2059-2076.

Oliveira MB et al. 2011. Liquid-liquid equilibria for the canola oil biodiesel + ethanol + glycerol system. Fuel 90, 2738-2745.

Oterhals A, Berntssen MHG. 2010. Effects of refining and removal of persistent organic pollutants by short-path distillation on nutritional quality and oxidative stability of fish oil. J. Agric. Food Chem. 58, 12250-12259.

Othmer DF, Tobias PE. 1942. Tie line correlation. Ind. Eng. Chem. 34, 693-696. 
Pawongrat R, Xu X, H-Kittikun A. 2008. Physico-enzymatic production of monoacylglycerols enriched with very-long-chain polyunsaturated fatty acids. J. Sci. Food Agric. 88, $256-262$.

Pawongrat $\mathrm{R}, \mathrm{Xu} \mathrm{X}, \mathrm{H}$-Kittikun A. 2007. Synthesis of monoacylglycerol rich in polyunsaturated fatty acids from tuna oil with immobilized lipase AK. Food Chem. 104, 251-258.

Pazhouhanmehr S et al. 2016. Oxidation kinetics of common Kilka ( Clupeonella cultiventris caspia ) oil in presence of bene oils ' unsaponifiable matter. Food Chem. 190, 748-754.

Perona JS, Ruiz-Gutierrez V. 1999. Characterization of the triacylglycerol molecular species of fish oil by reversed-phase high performance liquid chromatography. J. Liq. Chromatogr. 22, 1699-1714.

Pirestani S, Sahari MA, Barzegar M. 2010. Fatty Acids Changes during Frozen Storage in Several Fish Species from South Caspian Sea. J. Agric. Sci. Technol. 12, 321-329.

Pleiss J, Fischer M, Schmid RD. 1998. Anatomy of lipase binding sites: The scissile fatty acid binding site. Chem. Phys. Lipids 93, 67-80.

Podlaha O, Töregård B. 1982. A System for Identification of Triglycerides in Reverse Phase HPLC Chromatograms Based on Equivalent Carbon Numbers. J. High Resolut. Chromatogr. Chromatogr. Commun. 5, 553-557.

Podlaha O, Töregård B. 1989. Some new observations on the equivalent carbon numbers of triglycerides and relationship between changes in equivalent carbon number and molecular structure. J. Chromatogr. A 482, 215-226.

Prat D, Hayler J, Wells A. 2014. A survey of solvent selection guides. Green Chem. 16, 4546-4551.

Psota TL, Gebauer SK, Kris-Etherton P. 2006. Dietary Omega-3 Fatty Acid Intake and Cardiovascular Risk. Am J Cardio/|rOmega-3-Fatty Acids Cardiovasc. Dis. Reduct. 98, 
3-18.

Rebolleda S et al. 2012. Supercritical fluid extraction of corn germ oil: Study of the influence of process parameters on the extraction yield and oil quality. J. Supercrit. Fluids 72, 270-277.

Reid RC, Prausnitz JM, Poling BE. 1987. The Properties of Gases \& Liquids, McGraw-Hill, New York.

Remonatto D et al. 2015. Lipase-Catalyzed Glycerolysis of Soybean and Canola Oils in a Free Organic Solvent System Assisted by Ultrasound. Appl. Biochem. Biotechnol. 176, 850-862.

Rendón X, López-Munguía A, Castillo E. 2001. Solvent engineering applied to lipasecatalyzed glycerolysis of triolein. J. Am. Oil Chem. Soc. 78, 1061-1066.

Reyes-Labarta JA et al. 2009. Correlation of the liquid-liquid equilibrium data for specific ternary systems with one or two partially miscible binary subsystems. Fluid Phase Equilib. 278, 9-14.

Rezaei K, Temelli F, Jenab E. 2007. Effects of pressure and temperature on enzymatic reactions in supercritical fluids. Biotechnol. Adv. 25, 272-280.

Rezanka T, Mares P. 1991. Determination of plant triacylglycerols using capillary gas chromatography, high-performance liquid chromatography and mass spectrometry. J. Chromatogr. 542, 145-159.

Riddick JA, Bunger WB, Sakano TK. 1986. Organic Solvents, Physical Properties and Methods of Purification 4th ed., Jonh Wiley \& Sons, NY.

Riediger ND et al. 2009. Acids in Health and Disease. J. Am. Diet. Assoc. 109, 668-679.

Rodrigues CEC et al. 2005. Equilibrium data for systems composed by cottonseed oil+commercial linoleic acid+ethanol+water+tocopherols at 298.2K. Fluid Phase Equilib. 238, 193-203. 
Rodrigues CEC, Pessôa Filho PA, Meirelles AJA. 2004. Phase equilibrium for the system rice bran oil+fatty acid+ethanol+water +oryzanol+tocols. Fluid Phase Equilib. 216, 271283.

Rodrigues RC, Fernandez-Lafuente R. 2010. Lipase from Rhizomucor miehei as a biocatalyst in fats and oils modification. J. Mol. Catal. B Enzym. 66, 15-32.

Roh MK, Kim YD, Choi JS. 2015. Transesterification of fish oil by lipase immobilized in supercritical carbon dioxide. Fish. Sci. 81, 1113-1125.

Rossi $\mathrm{M}$ et al. 2003. The role of bleaching clays and synthetic silica in palm oil physical refining. Food Chem. 82, 291-296.

Rossi P et al. 2012. Fractionation and Concentration of Omega-3 by Molecular Distillation. in Eicosapentaenoic Acid: Sources, Health Effects and Role in Disease Prevention. Nova Science Publishers, Inc, pp. 177-203.

Rubio-Rodríguez $\mathrm{N}$ et al. 2012. Supercritical fluid extraction of fish oil from fish byproducts: A comparison with other extraction methods. J. Food Eng. 109, 238-248.

Ruiz-Sala P et al. 1996. Triglyceride composition of ewe, cow, and goat milk fat. J. Am. Oil Chem. Soc. 73, 283-293.

Sanz MT et al. 2002. Autocatalyzed and Ion-Exchange-Resin-Catalyzed Esterification Kinetics of Lactic Acid with Methanol. Ind. Eng. Chem. 41, 512-517.

Sathivel S, Huang J, Prinyawiwatkul W. 2008. Thermal properties and applications of the Arrhenius equation for evaluating viscosity and oxidation rates of unrefined pollock oil. J. Food Eng. 84, 187-193.

Schaefer A et al. 2003. Migration of lubricants from food packagings: Screening for lipid classes and quantitative estimation using normal-phase liquid chromatographic separation with evaporative light scattering detection. J. Chromatogr. A 1017, 107116. 
Sengwa RJ et al. 2010. Temperature dependent static dielectric constant and viscosity behaviour of glycerol-amide binary mixtures: Characterization of dominant complex structures in dielectric polarization and viscous flow processes. J. Mol. Liq.

Shahidi F, Wanasundara UN. 1998. Omega-3 fatty acid concentrates: nutritional aspects and production technologies. Trends Food Sci. Technol. 9, 230-240.

Shimada Y et al. 2003. Regiospecific Analysis by Ethanolysis of Oil with Immobilized Candida antarctica Lipase. Lipids 38, 1281-1286.

Sidhu KS. 2003. Health benefits and potential risks related to consumption of fish or fish oil. Regul. Toxicol. Pharmacol. 38, 336-344.

Da Silva $A E$ et al. 2011. Liquid-liquid equilibrium data for systems containing palm oil fractions + fatty acids + ethanol + water. J. Chem. Eng. Data 56, 1892-1898.

Silva Rodrigues J et al. 2017. Study of kinetics and thermodynamic parameters of the degradation process of biodiesel produced from fi sh viscera oil. Fuel Process. Technol. 161, 95-100.

Simopoulos AP. 2002. The importance of the ratio of omega-6/omega-3 essential fatty acids. Biomed. Pharmacother. 56, 365-379.

Singh AK, Mukhopadhyay M. 2016. Lipase-catalyzed glycerolysis of olive oil in organic solvent medium: Optimization using response surface methodology. Korean J. Chem. Eng. 33, 1247-1254.

Singh AK, Mukhopadhyay M. 2012. Olive oil glycerolysis with an immobilized lipase Candida antarctica in a solvent free system. Grasas y Aceites 63, 202-208.

Solaesa ÁG et al. 2014. Characterization of Triacylglycerol Composition of Fish Oils by Using Chromatographic Techniques. J. Oleo Sci. 63, 449-460.

Solaesa ÁG et al. 2015. Glycerolysis of sardine oil catalyzed by a water dependent lipase in different tert-alcohols as reaction medium. Grasas Aceites 66, 17-3495. 
Solaesa ÁG, Sanz MT, Beltrán S, et al. 2016. Kinetic study and kinetic parameters of lipasecatalyzed glycerolysis of sardine oil in a homogeneous medium. Chinese J. Catal. 37, 596-606.

Solaesa ÁG et al. 2013. Liquid-liquid equilibria for systems glycerol+sardine oil+tertalcohols. Fluid Phase Equilib. 356, 284-290.

Solaesa ÁG, Sanz MT, Falkeborg M, et al. 2016. Production and concentration of monoacylglycerols rich in omega-3 polyunsaturated fatty acids by enzymatic glycerolysis and molecular distillation. Food Chem. 190, 960-967.

Solaesa ÁG et al. 2017. Substrates emulsification process to improve lipase-catalyzed sardine oil glycerolysis in different systems. Evaluation of lipid oxidation of the reaction products. Food Res. Int. In Press.

Song JH, Fujimoto K, Miyazawa T. 2000. Polyunsaturated (n-3) fatty acids susceptible to peroxidation are increased in plasma and tissue lipids of rats fed docosahexaenoic acid-containing oils. J. Nutr. 130, 3028-3033.

Staby A, Mollerup JJ. 1993. Separation of constituents of fish oil using supercritical fluids: a review of experimental solubility, extraction, and chromatographic data. Fluid Phase Equilib. 91, 349-386.

Stamatis H, Xenakis A, Kolisis FN. 1999. Bioorganic reactions in microemulsions : the case of lipases. Biotechnol. Adv. 17, 293-318.

Stamatis H, Xenakis A, Kolisis FN. 1994. Studies on Enzyme Reuse and Product in Microemulsions ". Ann. N. Y. Acad. Sci. 1, 237-241.

Strieder MM et al. 2017. Bleaching optimization and winterization step evaluation in the refinement of rice bran oil. Sep. Purif. Technol. 175, 72-78.

Sullivan JC et al. 2015. Oxidation Rates of Triacylglycerol and Ethyl Ester Fish Oils. J. Am. Oil Chem. Soc. 92, 561-569. 
Sullivan JC, Budge SM, St-Onge M. 2011. Modeling the Primary Oxidation in Commercial Fish Oil Preparations. Lipids 46, 87-93.

Svensson J, Adlercreutz P. 2008. Identification of triacylglycerols in the enzymatic transesterification of rapeseed and butter oil. Eur. J. Lipid Sci. Technol. 110, 10071013.

Tai HP, Brunner G. 2011. Mono-and di-acylglycerol synthesis in CO 2 -expanded acetone. J. Supercrit. Fluids 59, 87-91.

Tamir A. 1981. Correlation of vapor-liquid equilibria in systems showing homoazeotropy or heteroazeotropy using expressions for the total pressure and temperature as direct functions of vapor composition. Chem. Eng. Sci. 36, 1467-1473.

Tan T, Yin C. 2005. The mechanism and kinetic model for glycerolysis by 1,3 position specific lipase from Rhizopus arrhizus. Biochem. Eng. J. 25, 39-45.

Tao M et al. 2013. Enzymatic Synthesis of Dipalmitin in Supercritical Carbon Dioxide and Mechanism Study. Ind. Eng. Chem. Res. 52, 13528-13535.

Temelli F, King JW, List GR. 1996. Conversion of Oils to Monoglycerides by Glycerolysis in Supercritical Carbon Dioxide Media. JAOCS 73, 699-706.

Toro-Vazquez JF, Garcia-L OE, Guerrero-E LL. 1991. Adsorption Isotherms of Squash ( Cucurbita moschata) Seed Oil on Activated Carbon. J. Am. Oil Chem. Soc. 68, 596599.

Torres C, Lin B, Hill CG. 2002. Lipase-catalyzed glycerolysis of an oil rich in eicosapentaenoic acid residues. Biotechnol. Lett. 24, 667-673.

Tou JC et al. 2011. Different sources of omega-3 polyunsaturated fatty acids affects apparent digestibility, tissue deposition, and tissue oxidative stability in growing female rats. Lipids Health Dis. 10, 179.

Üstün G et al. 1997. Enzymatic hydrolysis of anchovy oil: Production of glycerides enriched 
in polyunsaturated fatty acids. Appl. Biochem. Biotechnol. 68, 171-186.

Valério A, Fiametti KG, et al. 2009. Enzymatic production of mono- and diglycerides in compressed n-butane and AOT surfactant. J. Supercrit. Fluids 49, 216-220.

Valério A, Krüger RL, et al. 2009. Kinetics of solvent-free lipase-catalyzed glycerolysis of olive oil in surfactant system. J. Agric. Food Chem. 57, 8350-8356.

Valério A et al. 2010. Optimization of mono and diacylglycerols production from enzymatic glycerolysis in solvent-free systems. Bioprocess Biosyst. Eng. 33, 805-812.

Voll F et al. 2011. Kinetic modeling of lipase-catalyzed glycerolysis of olive oil. Biochem. Eng. J. 56, 107-115.

Wanasundara U. 2011. Preparative and industrial-scale isolation and purification of omega-3 polyunsaturated fatty acids from marine sources. in Handbook of seafood quality, safety and health applications. pp. 464-475.

Wani AL, Bhat SA, Ara A. 2015. Omega-3 fatty acids and the treatment of depression: a review of scientific evidence. Integr. Med. Res. 4, 132-141.

Weber N, Mukherjee KD. 2004. Solvent-free lipase-catalyzed preparation of diacylglycerols. J. Agric. Food Chem. 52, 5347-5353.

Wewer V, Dörmann P, Hölzl G. 2013. Analysis and Quantification of Plant Membrane Lipids by Thin-Layer Chromatography and Gas Chromatography. in Methods in Molecular Biology. pp. 69-78.

Wijesundera $\mathrm{C}$ et al. 2008. Docosahexaenoic acid is more stable to oxidation when located at the sn-2 position of triacylglycerol compared to sn-1(3). J. Am. Oil Chem. Soc. 85, $543-548$.

Wongsakul S et al. 2003. Synthesis of 2-monoglycerides by alcoholysis of palm oil and tuna oil using immobilized lipases. Eur. J. Lipid Sci. Technol. 105, 68-73. 
Wu K et al. 2016. Enriched endogenous n-3 polyunsaturated fatty acids alleviate cognitive and behavioral deficits in a mice model of Alzheimer's disease. Neuroscience 333, 345-355.

Xu X. 2003. Engineering of enzymatic reactions and reactors for lipid modification and synthesis. Eur. J. Lipid Sci. Technol. 105, 289-304.

Xu X. 2000. Production of specific-structured triacylglycerols by lipase-catalyzed reactions: a review. Eur. J. Lipid Sci. Technol. 102, 287-303.

$\mathrm{Xu} \mathrm{X}$ et al. 2002. Purification and deodorization of structured lipids by short path distillation. Eur. J. Lipid Sci. Technol. 104, 745-755.

Yang $T$ et al. 2005a. Enzymatic production of monoacylglycerols containing polyunsaturated fatty acids through an efficient glycerolysis system. J. Agric. Food Chem. 53, 1475-1481.

Yang T et al. 2005b. Monoacylglycerol synthesis via enzymatic glycerolysis using a simple and efficient reaction system. J. Food Lipids 12, 299-312.

Yeoh CM et al. 2009. Influence of silica gel in production of diacylglycerol via enzymatic glycerolysis of palm olein. Eur. J. Lipid Sci. Technol. 111, 599-606.

Yeoh CM et al. 2014. Molecular distillation and characterization of diacylglycerol-enriched palm olein. Eur. J. Lipid Sci. Technol. 116, 1-10.

Yin H, Sathivel S. 2010. Physical Properties and Oxidation Rates of Unrefined Menhaden Oil ( Brevoortia patronus ). J. Food Sci. 75, 163-168.

Young FVK. 1986. Fish Oil Bulletin No. 18.

Zaks A, Klibanov AM. 1985. Enzyme-catalyzed processes in organic solvents. Biochemistry 82, 3192-3196.

Zeng FK et al. 2010. Enzymatic production of monoacylglycerols with camellia oil by the 
glycerolysis reaction. J. Am. Oil Chem. Soc. 87, 531-537.

Zha B et al. 2014. Production of glycerol monolaurate-enriched monoacylglycerols by lipase-catalyzed glycerolysis from coconut oil. Eur. J. Lipid Sci. Technol. 116, 328335.

Zhang G, Liu J, Liu Y. 2013. Concentration of omega-3 polyunsaturated fatty acids from oil of schizochytrium limacinum by molecular distillation: Optimization of technological conditions. Ind. Eng. Chem. Res. 52, 3918-3925.

Zhang H. 2007. Evaluation of Practical Process Aspects for Lipozyme TL IM Catalyzed Bulk Fat Modification in a Batch Reactor. Open Biotechnol. J. 1, 72-80.

Zheng P et al. 2014. Production of diacylglycerol-mixture of regioisomers with high purity by two-step enzymatic reactions combined with molecular distillation. JAOCS, J. Am. Oil Chem. Soc. 91, 251-259.

Zhong $\mathrm{N}$ et al. 2009. An Efficient Binary Solvent Mixture for Monoacylglycerol Synthesis by Enzymatic Glycerolysis. JAOCS, J. Am. Oil Chem. Soc. 86, 783-789.

Zhu Q et al. 2011. A two-stage enzymatic process for synthesis of extremely pure high oleic glycerol monooleate. Enzyme Microb. Technol. 48, 143-147. 



\section{CURRICULUM VITAE}

\section{Ángela García Solaesa}

Born on June 13, 1986 in Soria (Spain)

\section{Education}

2013-2017 PhD student in the program "Advances on Food Science and Biotechnology", University of Burgos, Spain.

2010-2012 Master in Food Biotechnology and Safety, University of Burgos, Spain.

2008-2010 Bachelor's Degree in Food Science and Technology, Autonomous University of Madrid, Spain.

2004-2008 Associate's Degree in Human Nutrition and Dietetics, Complutense University of Madrid, Spain.

Employment

2013-2017 University of Burgos PhD fellowship in the Department of Biotechnology and Food Science of University of Burgos, Spain.

2012-2013 Research technical assistant in the Department of Biotechnology and Food Science of University of Burgos, Spain.

2010-2011 Research Scholar in the Department of Biotechnology and Food Science of University of Burgos, Spain.

Shorts research stays in Universities and/or Institutes of Research

2017 REQUIMTE - Faculty of Pharmacy, University of Porto (Portugal). 2 months.

2015 School of Chemical Engineering, University of Birmingham (UK). 1 week. 
2014 INCUBA Science Park, University of Aarhus (Denmark). 3 months.

2012 Faculty of Pharmacy, University of Jordan (Jordan). 1 month.

2011-2012 Faculty of Bioengineering, University of Gent (Belgium). 5 months.

\section{Publications}

Solaesa ÁG et al. 2017. Substrates emulsification process to improve lipase-catalyzed sardine oil glycerolysis in different systems. Evaluation of lipid oxidation of the reaction products. Food Res. Int. In Press.

Solaesa ÁG et al. 2016. Production and concentration of monoacylglycerols rich in omega- 3 polyunsaturated fatty acids by enzymatic glycerolysis and molecular distillation. Food Chem. 190, 960-967.

Solaesa ÁG et al. 2016. Kinetic study and kinetic parameters of lipase-catalyzed glycerolysis of sardine oil in a homogeneous medium. Chinese J. Catal. 37, 596606.

Solaesa ÁG et al. 2015. Glycerolysis of sardine oil catalyzed by a water dependent lipase in different tert-alcohols as reaction medium. Grasas Aceites 66, 17-3495.

Solaesa ÁG et al. 2014. Characterization of Triacylglycerol Composition of Fish Oils by Using Chromatographic Techniques. J. Oleo Sci. 63, 449-460.

Solaesa ÁG et al. 2013. Liquid-liquid equilibria for systems glycerol+sardine oil+tertalcohols. Fluid Phase Equilib. 356, 284-290.

\section{Oral communications}

Ángela García Solaesa, Rodrigo Melgosa, María Teresa Sanz, Sagrario Beltrán. “Lipasecatalyzed glycerolysis of sardine oil in supercritical carbon dioxide media" in $15^{\text {th }}$ European Meeting on Supercritical Fluids, May 2016, Essen (Alemania). 
Ángela García Solaesa, Rodrigo Melgosa, María Teresa Sanz, Sagrario Beltrán. “Producción de concentrados de acilglicéridos ricos en ácidos grasos omega-3" in II Jornada de Doctorandos de la Univerisdad de Burgos, December 2015, Burgos (Spain).

Ángela García Solaesa, Rodrigo Melgosa, María Teresa Sanz, Sagrario Beltrán. "Study of Different Fractionation Techniques in the Concentration of Omega-3 as Acylglycerides" in I Congreso Nacional de Jóvenes Investigadores en Ciencia, Ingeniería y Tecnología de los Alimentos (JICITA), November 2015, Palencia (Spain).

Poster presentations

Ángela García Solaesa, Rodrigo Melgosa, María Teresa Sanz, Sagrario Beltrán Alba E. Illera. "Comparison of Glycerolysis of Sardine Oil by Lipozyme 435 in Solvent free and SC-CO2 media" in $16^{\text {th }}$ European Meeting on Supercritical Fluids, April 2017, Lisbon (Portugal).

Ángela García Solaesa, Maria Teresa Sanz, Rodrigo Melgosa, Mia Falkeborg, Sagrario Beltrán, Zheng Guo. "Concentration of Monoacylglycerols Rich in Omega-3 Polyunsaturated Fatty Acids by Enzymatic Glycerolysis and Molecular Distillation" in ECC10+ECAB3+EPIC5, October 2015, Nice (France).

Ángela García Solaesa, Rodrigo Melgosa, Maria Teresa Sanz, Sagrario Beltrán. "Supercritical CO2 Fractionation of Fish Oil Derivatives For Obtaining Omega-3 Concentrates" in VIII Reunión de Expertos en Fluidos Comprimidos (FLUCOMP), September 2015, Cadiz (Spain).

Ángela García Solaesa, Silvia Liliana Bucio, María Teresa Sanz, Sagrario Beltrán. “Liquid liquid equilibrium for glycerolisis systems of sardine oil” in XXXIV Reunión Bienal Real Sociedad Española de Química, September 2013, Santander (Spain). 